\title{
2021 ESC/EACTS Guidelines for the management of valvular heart disease
}

\author{
Developed by the Task Force for the management of valvular heart disease of the European Society of Cardiology \\ (ESC) and the European Association for Cardio-Thoracic Surgery (EACTS)

\begin{abstract}
Authors/Task Force Members: Alec Vahanian* (ESC Chairperson) (France), Friedhelm Beyersdorf*l (EACTS Chairperson) (Germany), Fabien Praz (ESC Task Force Coordinator) (Switzerland), Milan Milojevicl (EACTS Task Force Coordinator) (Serbia), Stephan Baldus (Germany), Johann Bauersachs (Germany), Davide Capodanno (Italy), Lenard Conradi (Germany), Michele De Bonis ${ }^{l}$ (Italy), Ruggero De Paulis ${ }^{l}$ (Italy), Victoria Delgado (Netherlands), Nick Freemantle ${ }^{l}$ (United Kingdom), Martine Gilard (France), Kristina H. Haugaa (Norway), Anders Jeppsson' (Sweden), Peter Jüni (Canada), Luc Pierard (Belgium), Bernard D. Prendergast (United Kingdom), J. Rafael Sádabal (Spain), Christophe Tribouilloy (France), Wojtek Wojakowski (Poland), ESC/EACTS Scientific Document Group
\end{abstract}

${ }^{1}$ Representing the European Association for Cardio-Thoracic Surgery (EACTS)

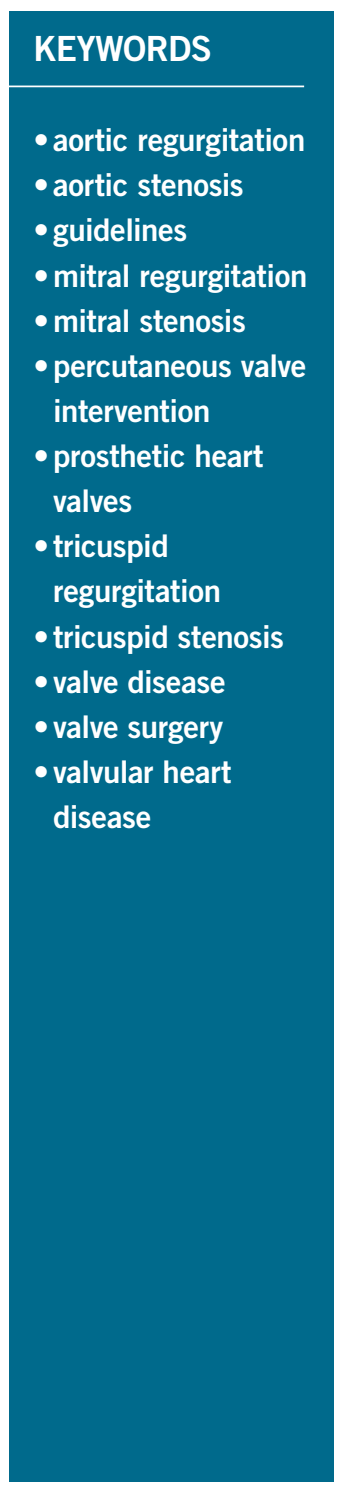

\begin{abstract}
Author/Task Force Member affiliations: listed in Author information. ESC Clinical Practice Guidelines Committee (CPG): listed in the Appendix. EACTS Council: listed in the Appendix.
\end{abstract}

Associations: Association for Acute CardioVascular Care (ACVC), European Association of Cardiovascular Imaging (EACVI), European Association of Percutaneous Cardiovascular Interventions (EAPCI), European Heart Rhythm Association (EHRA), Heart Failure Association (HFA).

Councils: Council on Valvular Heart Disease.

Working Groups: Cardiovascular Surgery, Thrombosis.

Vahanian A, Beyersdorf F, Praz F, et al, 2021 ESC/EACTS Guidelines for the management of valvular heart disease: Developed by the Task Force for the management of valvular heart disease of the European Society of Cardiology (ESC) and the European Association for Cardio-Thoracic Surgery (EACTS), European Heart Journal, 2021; doi:10.1093/eurheartj/ehab395. Reprinted by permission of Oxford University Press on behalf of the European Society of Cardiology.

This publication comprises a reprint of the official full text version of the "2021 ESC/EACTS Guidelines for the management of valvular heart disease" ("ESC Guidelines") originally published in English in the European Heart Journal by Oxford University Press under licence from the European Society of Cardiology ("ESC").

This publication is for personal and educational use only. No commercial use is authorized. No part of this publication or the original ESC Guidelines from which it is derived may be translated or reproduced in any form without written permission from the ESC. Permission may be obtained upon submission of a written request to Oxford University Press, the publisher of the European Heart Journal and the party authorized to handle such permissions by the ESC. Merck Group has obtained permission to publish this reprint and to distribute it to health professionals globally.

All rights reserved; no part of this publication may be reproduced, stored in a retrieval system, or transmitted in any form or by any means, electronic, mechanical, photocopying, recording, or otherwise without the prior written permission of Oxford University Press or its licensee Oxford Publishing Limited ("OPL").

For permissions, please contact journals.permissions@oup.com

The ESC Guidelines represent the views of the ESC and were produced after careful consideration of the scientific and medical knowledge and the evidence available at the time of their dating.

The ESC is not responsible in the event of any contradiction, discrepancy and/or ambiguity between the ESC Guidelines and any other official recommendations or guidelines issued by the relevant public health authorities, in particular in relation to good use of health care or therapeutic strategies. Health professionals are encouraged to take the ESC Guidelines fully into account when exercising their clinical judgment as well as in the determination and the implementation of preventive, diagnostic or therapeutic medical strategies. However, the ESC Guidelines do not override in any way whatsoever the individual responsibility of health professionals to make appropriate and accurate decisions in consideration of each patient's health condition and in consultation with that patient and the patient's caregiver where appropriate and/or necessary. Nor do the ESC Guidelines exempt health professionals from taking careful and full consideration of the relevant official updated recommendations or guidelines issued by the competent public health authorities in order to manage each patient's case in light of the scientifically accepted data pursuant to their respective ethical and professional obligations. It is also the health professional's responsibility to verify the applicable rules and regulations relating to drugs and medical devices at the time of prescription.

\footnotetext{
* Corresponding authors: Alec Vahanian, UFR Medecine, Université de Paris, site Bichat, 16 rue Huchard, 75018 Paris, France; and LVTS INSERM U1148, GH Bichat, 46, rue Henri Huchard, 75018 Paris, France. Tel: + 33663155668.

E-mail: alec.vahanian@gmail.com; Friedhelm Beyersdorf, Department of Cardiovascular Surgery, University Heart Center,

University Hospital Freiburg, Germany; and Medical Faculty of the Albert-Ludwigs-University, Freiburg, Germany,

Hugstetterstr. 55, D-79106 Freiburg, Germany. Tel: +49 761270 28180. E-mail: friedhelm.beyersdorf@uniklinik-freiburg.de
} 
Document Reviewers: Franz-Josef Neumann (ESC Review Coordinator) (Germany), Patrick Myers ${ }^{1}$ (EACTS Review Coordinator) (Switzerland), Magdy Abdelhamid (Egypt), Stephan Achenbach (Germany), Riccardo Asteggiano (Italy), Fabio Barili ${ }^{1}$ (Italy), Michael A. Borger (Germany), Thierry Carrel ${ }^{1}$ (Switzerland), Jean-Philippe Collet (France), Dan Foldager (Denmark), Gilbert Habib (France), Christian Hassager (Denmark), Alar Irs $^{1}$ (Estonia), Bernard Iung (France), Marjan Jahangiri ${ }^{1}$ (United Kingdom), Hugo A. Katus (Germany), Konstantinos C. Koskinas (Switzerland), Steffen Massberg (Germany), Christian E. Mueller (Switzerland), Jens Cosedis Nielsen (Denmark), Philippe Pibarot (Canada), Amina Rakisheva (Kazakhstan), Marco Roffi (Switzerland), Andrea Rubboli (Italy), Evgeny Shlyakhto (Russia), Matthias Siepe ${ }^{1}$ (Germany), Marta Sitges (Spain), Lars Sondergaard (Denmark), Miguel Sousa-Uva ${ }^{1}$ (Portugal), Guiseppe Tarantini (Italy), Jose Luis Zamorano (Spain)

${ }^{1}$ Representing the European Association for Cardio-Thoracic Surgery (EACTS)

All experts involved in the development of these guidelines have submitted declarations of interest.

These have been compiled in a report and published in a supplementary document simultaneously to the guidelines. The report is also available on the ESC website www.escardio.org/guidelines.

For the Supplementary Data which include background information and detailed discussion of the data that have provided the basis for the guidelines see European Heart Journal online.

ESC subspecialty communities having participated in the development of this document:

This article has been co-published with permission in the European Heart Journal and European Journal of Cardio-Thoracic Surgery. VC the European Society of Cardiology and the European Association for Cardio-Thoracic Surgery 2021. All rights reserved. The articles are identical except for minor stylistic and spelling differences in keeping with each journal's style. Either citation can be used when citing this article. For permissions, please email journals.permissions@oup.com.

\section{Table of contents}

Abbreviations and acronyms

1 Preamble

2 Introduction 1131

2.1 Why do we need new guidelines on valvular heart disease?

2.2 Methodology

2.3 Content of these guidelines

2.4 New format of the guidelines

2.5 How to use these guidelines

3 General comments

3.1 Concepts of Heart Team and Heart Valve Centre

3.2 Patient evaluation

3.2.1 Clinical evaluation

3.2.2 Echocardiography

3.2.3 Other non-invasive investigations

3.2.4 Invasive investigations

3.2.5 Assessment of comorbidity

3.3 Risk stratification

3.3.1 Risk scores

3.3.2 Other factors

3.4 Patient-related aspects

3.5 Local resources

3.6 Management of associated conditions

3.6.1 Coronary artery disease

3.6.2 Atrial fibrillation

3.7 Endocarditis prophylaxis

3.8 Prophylaxis for rheumatic fever

4 Aortic regurgitation. 1141

\subsection{Evaluation}

\subsubsection{Echocardiography}

4.1.2 Computed tomography and cardiac magnetic resonance

4.2 Indications for intervention

4.3 Medical therapy
4.4 Serial testing

4.5 Special patient populations

5 Aortic stenosis

5.1 Evaluation

5.1.1 Echocardiography

5.1.2 Additional diagnostic and prognostic parameters

5.1.3 TAVI diagnostic workup

5.2 Indications for intervention (SAVR or TAVI)

5.2.1 Symptomatic aortic stenosis

5.2.2 Asymptomatic aortic stenosis

5.2.3 The mode of intervention

5.3 Medical therapy

5.4 Serial testing

5.5 Special patient populations

6 Mitral regurgitation....

6.1 Primary mitral regurgitation

\subsubsection{Evaluation}

6.1.2 Indications for intervention

6.1.3 Medical therapy

6.1.4 Serial testing

6.1.5 Special populations

6.2 Secondary mitral regurgitation

\subsubsection{Evaluation}

6.2.2 Medical therapy

6.2.3 Indications for intervention

7 Mitral stenosis

7.1 Rheumatic mitral stenosis

\subsubsection{Evaluation}

7.1.2 Indications for intervention

7.1.3 Medical therapy

7.1.4 Serial testing

7.1.5 Special patient populations

7.2 Degenerative mitral stenosis with mitral annular calcification 


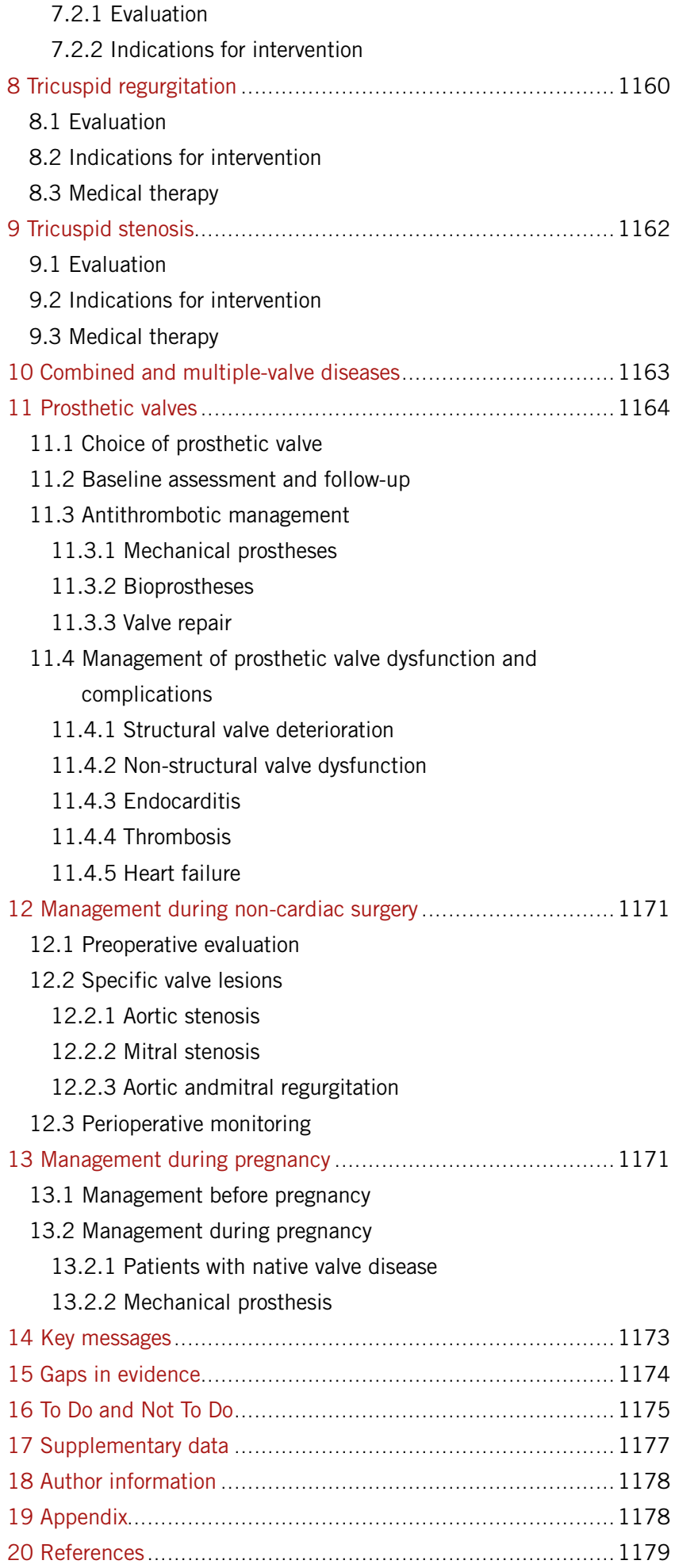

\section{List of tables}

Table 1. Classes of recommendations.

Table 2. Levels of evidence.

Table 3. What is new.

Table 4. Requirements for a Heart Valve Centre.

Table 5. Echocardiographic criteria for the definition of severe aortic valve regurgitation.
Table 6. Clinical, anatomical and procedural factors that influence the choice of treatment modality for an individual patient.

Table 7. Severe mitral regurgitation criteria based on 2D echocardiography.

Table 8. Contraindications for percutaneous mitral commissurotomy in rheumatic mitral stenosisa.

Table 9. Echocardiographic criteria for grading severity of tricuspid regurgitation.

Table 10. Target international normalized ratio for mechanical prostheses.

\section{List of figures}

Figure 1 (Central illustration). Patient-centred evaluation for intervention.

Figure 2 Management of patients with aortic regurgitation.

Figure 3. Integrated imaging assessment of aortic stenosis.

Figure 4. Management of patients with severe aortic stenosis.

Figure 5. Management of patients with severe chronic primary mitral regurgitation.

Figure 6. Management of patients with chronic severe secondary mitral regurgitation.

Figure 7. Management of clinically significant rheumatic mitral stenosis (MVA $\leq 1.5 \mathrm{~cm}^{2}$ ).

Figure 8. Management of tricuspid regurgitation (TR).

Figure 9. Antithrombotic therapy for valve prostheses.

Figure 10. Management of left-sided obstructive and non-obstructive mechanical prosthetic thrombosis.

Figure 11. Management of non-cardiac surgery (NCS) in patients with severe aortic stenosis.

\section{Tables of recommendations}

Recommendations for management of CAD in patients with VHD. Recommendations on management of atrial fibrillation in patients with native VHD.

Recommendations on indications for surgery in (A) severe aortic regurgitation and (B) aortic root or tubular ascending aortic aneurysm (irrespective of the severity of aortic regurgitation).

Recommendations on indications for interventiona in symptomatic (A) and asymptomatic (B) aortic stenosis and recommended mode of intervention (C).

Recommendations on indications for intervention in severe primary mitral regurgitation.

Recommendations on indications for mitral valve intervention in chronic severe secondary mitral regurgitation.

Recommendations on indications for percutaneous mitral commissurotomy and mitral valve surgery in clinically significant (moderate or severe) mitral stenosis (valve area $\leq 1.5 \mathrm{~cm}^{2}$ ).

Recommendations on indications for intervention in tricuspid valve disease.

Recommendations for prosthetic valve selection.

Recommendations for management of antithrombotic therapy after prosthetic valve implantation or valve repair in the perioperative and postoperative periods.

Recommendations on management of prosthetic valve dysfunction. 


\section{Abbreviations and acronyms}

2D Two-dimensional

3D Three-dimensional

ACEI Angiotensin-converting enzyme inhibitor

ACS Acute coronary syndrome

AF Atrial fibrillation

ARB Angiotensin receptor blocker

ARC-HBR Academic Research Consortium-High Bleeding Risk

ASA Acetylsalicylic acid

AVA Aortic valve area

BAV Balloon aortic valvuloplasty

BHV Biological heart valve

BVF Bioprosthetic valve failure

BNP B-type natriuretic peptide

BP Blood pressure

BSA Body surface area

CABG Coronary artery bypass grafting

CAD Coronary artery disease

CCT Cardiac computed tomography

CI Confidence interval

CMR Cardiac magnetic resonance

CRT Cardiac resynchronization therapy

CT Computed tomography

DAPT Dual antiplatelet therapy

DPm Mean pressure gradient

DSE Dobutamine stress echocardiography

DVI Doppler velocity index/dimensionless index

EACTS European Association for Cardio-Thoracic Surgery

ECG Electrocardiogram

EDV End-diastolic velocity

EROA Effective regurgitant orifice area

ESC European Society of Cardiology

EuroSCORE European System for Cardiac Operative Risk Evaluation

FFP Fresh frozen plasma

GDMT Guideline-directed medical treatment therapy

HALT Hypo-attenuated leaflet thickening

HTx Heart transplantation

INR International normalized ratio

i.v. Intravenous

LA Left atrium/left atrial

LAA Left atrial appendage

LMWH Low-molecular-weight heparin

LV Left ventricle/left ventricular

LVAD Left ventricular assist devices

LVEDD Left ventricular end-diastolic diameter

LVEF Left ventricular ejection fraction

LVESD Left ventricular end-systolic diameter

LVOT Left ventricular outflow tract

MAC Mitral annular calcification

MHV Mechanical heart valve

MIDA Mitral Regurgitation International Database

MVA Mitral valve area
Non-cardiac surgery

NOAC Non-vitamin $\mathrm{K}$ antagonist oral anticoagulant

NYHA New York Heart Association

OAC Oral anticoagulation

PCC Prothrombin complex concentration

PCI Percutaneous coronary intervention

PET Positron emission tomography

PISA Proximal isovelocity surface area

PMC Percutaneous mitral commissurotomy

PMR Primary mitral regurgitation

PPM Patient-prosthesis mismatch

PROM Predicted risk of mortality

RCT Randomized controlled trial

RV Right ventricle/right ventricular

SAPT Single antiplatelet therapy

SAVR Surgical aortic valve replacement

SMR Secondary mitral regurgitation

SVD Structural valve deterioration

SPAP Systolic pulmonary arterial pressure

STS Society of Thoracic Surgeons

SVi Stroke volume index

TAPSE Tricuspid annular pulmonary systolic excursion

TAVI Transcatheter aortic valve implantation

TE Thromboembolism

TEER Transcatheter edge-to-edge repair

TTVI Transcatheter tricuspid valve intervention

TOE Transoesophageal echocardiography

TTE Transthoracic echocardiography

TVI Time-velocity integral

TVR Tricuspid valve replacement or repair

UFH Unfractionated heparin

VHD Valvular heart disease

VKA Vitamin $\mathrm{K}$ antagonist

$\mathbf{V}_{\max }$ Peak transvalvular velocity

\section{Preamble}

Guidelines summarize and evaluate available evidence with the aim of assisting health professionals in proposing the best management strategies for an individual patient with a given condition. Guidelines and their recommendations should facilitate decision making of health professionals in their daily practice. However, the final decisions concerning an individual patient must be made by the responsible health professional(s) in consultation with the patient and caregiver as appropriate.

A great number of guidelines have been issued in recent years by the European Society of Cardiology (ESC) and its partners such as the European Association for Cardio-Thoracic Surgery (EACTS), as well as by other societies and organizations. Because of their impact on clinical practice, quality criteria for the development of guidelines have been established in order to make all decisions transparent to the user. The recommendations for formulating and issuing ESC Guidelines can be found on the ESC website (https://www.escardio.org/Guidelines). The ESC Guidelines 
Table 1. Classes of recommendations.

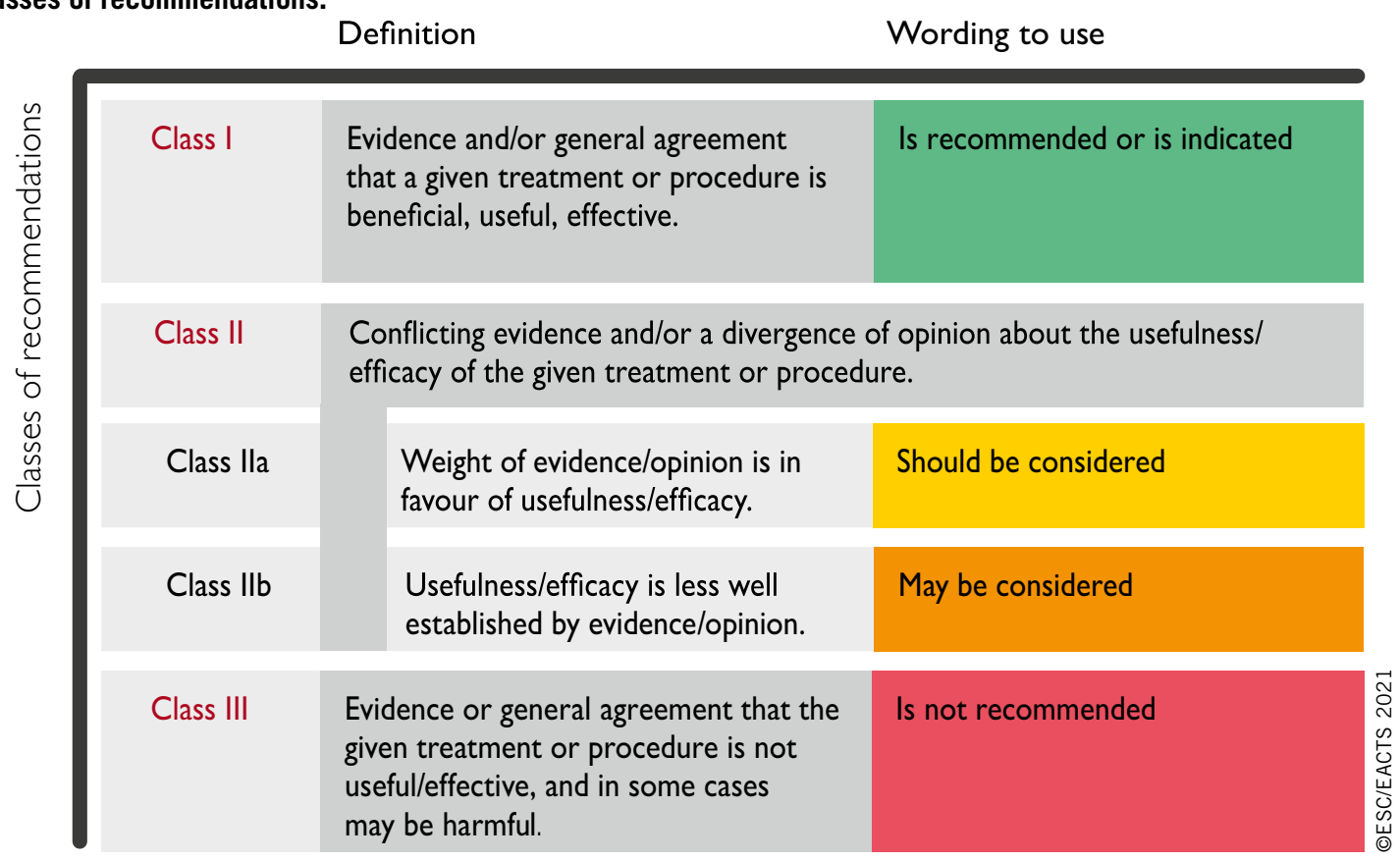

represent the official position of the ESC on a given topic and are regularly updated.

In addition to the publication of Clinical Practice Guidelines, the ESC carries out the EURObservational Research Programme of international registries of cardiovascular diseases and interventions which are essential to assess diagnostic/therapeutic processes, use of resources and adherence to guidelines. These registries aim at providing a better understanding of medical practice in Europe and around the world, based on high-quality data collected during routine clinical practice.

The Members of this Task Force were selected by the ESC and EACTS, including representation from relevant ESC and EACTS sub-specialty groups, in order to represent professionals involved with the medical care of patients with this pathology. Selected experts in the field undertook a comprehensive review of the published evidence for management of a given condition according to ESC Clinical Practice Guidelines Committee (CPG). A critical evaluation of diagnostic and therapeutic procedures was performed, including assessment of the risk-benefit ratio. The level of evidence and the strength of the recommendation of particular management options were weighed and graded according to predefined scales, as outlined below.

The experts of the writing and reviewing panels provided declaration of interest forms for all relationships that might be perceived as real or potential sources of conflicts of interest. Their declarations of interest were reviewed according to the ESC declaration of interest rules and can be found on the ESC website (http://www.escardio. org/guidelines) and have been compiled in a report and published in a supplementary document simultaneously to the guidelines.

This process ensures transparency and prevents potential biases in the development and review processes. Any changes in declarations of interest that arise during the writing period were notified

\section{Table 2. Levels of evidence.}




to the ESC and updated. The Task Force received its entire financial support from the ESC and EACTS without any involvement from the healthcare industry.

The ESC CPG supervises and coordinates the preparation of new guidelines. The Committee is also responsible for the endorsement process of these guidelines. The ESC Guidelines undergo extensive review by the CPG and external experts. After appropriate revisions the guidelines are signed-off by all the experts involved in the Task Force. The finalized document is signed-off by the CPG for publication in the European Heart Journal and the European Journal of Cardio-Thoracic Surgery. The guidelines were developed after careful consideration of the scientific and medical knowledge and the evidence available at the time of their dating.

The task of developing ESC/EACTS Guidelines also includes the creation of educational tools and implementation programmes for the recommendations including condensed pocket guideline versions, summary slides, summary cards for non-specialists and an electronic version for digital applications (smartphones, etc.). These versions are abridged and thus, for more detailed information, the user should always access to the full text version of the guidelines, which is freely available via the ESC and EACTS website and hosted on the EHJ and EJCTS website. The National Cardiac Societies of the ESC are encouraged to endorse, adopt, translate and implement all ESC Guidelines. Implementation programmes are needed because it has been shown that the outcome of disease may be favourably influenced by the thorough application of clinical recommendations.

Health professionals are encouraged to take the ESC/EACTS Guidelines fully into account when exercising their clinical judgment, as well as in the determination and the implementation of preventive, diagnostic or therapeutic medical strategies. However, the ESC/EACTS Guidelines do not override in any way whatsoever the individual responsibility of health professionals to make appropriate and accurate decisions in consideration of each patient's health condition and in consultation with that patient or the patient's caregiver where appropriate and/or necessary. It is also the healthcare professional's responsibility to verify the rules and regulations applicable in each country to drugs and devices at the time of prescription.

\section{Introduction}

\subsection{WHY DO WE NEED NEW GUIDELINES ON VALVULAR HEART DISEASE?}

Since the publication of the previous version of the guidelines on the management of valvular heart disease (VHD) in 2017, new evidence has accumulated, particularly on the following topics:

- Epidemiology: the incidence of the degenerative aetiology has increased in industrialized countries while, unfortunately, rheumatic heart disease is still too frequently observed in many parts of the world. ${ }^{1-3}$

- Current practices regarding interventions and medical management have been analysed in new surveys at the national and European level.
- Non-invasive evaluation using three-dimensional (3D) echocardiography, cardiac computed tomography (CCT), cardiac magnetic resonance (CMR), and biomarkers plays a more and more central role.

- New definitions of severity of secondary mitral regurgitation (SMR) based on the outcomes of studies on intervention.

- New evidence on anti-thrombotic therapies leading to new recommendations in patients with surgical or transcatheter bioprostheses for bridging during perioperative periods and over the long term. The recommendation for non- vitamin $\mathrm{K}$ antagonist oral anticoagulants (NOACs) was reinforced in patients with native valvular disease, except for significant mitral stenosis, and in those with bioprostheses.

- Risk stratification for the timing of intervention. This applies mostly to (i) the evaluation of progression in asymptomatic patients based on recent longitudinal studies mostly in aortic stenosis, and (ii) interventions in high-risk patients in whom futility should be avoided. Regarding this last aspect, the role of frailty is outlined.

- Results and indication of intervention:

- The choice of the mode of intervention: current evidence reinforces the critical role of the Heart Team, which should integrate clinical, anatomical, and procedural characteristics beyond conventional scores, and informed patient's treatment choice. Surgery: increasing experience and procedural safety led to expansion of indications toward earlier intervention in asymptomatic patients with aortic stenosis, aortic regurgitation or mitral regurgitation and stress the preference for valve repair when it is expected to be durable. A particular emphasis is put on the need for more comprehensive evaluation and earlier surgery in tricuspid regurgitation.

- Transcatheter techniques: (i) Concerning transcatheter aortic valve implantation (TAVI), new information from randomized studies comparing TAVI vs. surgery in low-risk patients with a follow-up of 2 years has led to a need to clarify which types of patients should be considered for each mode of intervention. (ii) Transcatheter edge-to-edge repair (TEER) is increasingly used in SMR and has been evaluated against optimal medical therapy resulting in an upgrade of the recommendation. (iii) The larger number of studies on transcatheter valvein-valve implantation after failure of surgical bioprostheses served as a basis to upgrade its indication. (iv) Finally, the encouraging preliminary experience with transcatheter tricuspid valve interventions (TTVI) suggests a potential role of this treatment in inoperable patients, although this needs to be confirmed by further evaluation.

The new evidence described above made a revision of the recommendations necessary.

\subsection{METHODOLOGY}

In preparation of the 2021 VHD Guidelines, a methodology group has been created for the first time, to assist the Task Force for the collection and interpretation of the evidence supporting specific 
Table 3. What is new.

\begin{tabular}{|c|c|c|c|c|}
\hline New or Revised & Recommendations in 2017 version & Class & Recommendations in 2021 version & Class \\
\hline \multicolumn{5}{|c|}{ Section 3. Management of atrial fibrillation in patients with native VHD } \\
\hline Revised & $\begin{array}{l}\text { Surgical excision or external clipping of the } \\
\text { LAA may be considered in patients undergoing } \\
\text { valve surgery. }\end{array}$ & Ilb & $\begin{array}{l}\text { LAA occlusion should be considered to reduce } \\
\text { the thromboembolic risk in patients with AF } \\
\text { and a } \mathrm{CHA}_{2} \mathrm{DS}_{2} \text { VASc score } \geq 2 \text { undergoing valve } \\
\text { surgery. }\end{array}$ & Ila \\
\hline Revised & $\begin{array}{l}\text { NOACs should be considered as an alternative } \\
\text { to VKAs in patients with aortic stenosis, aortic } \\
\text { regurgitation and mitral regurgitation } \\
\text { presenting with AF. }\end{array}$ & Ila & $\begin{array}{l}\text { For stroke prevention in } \mathrm{AF} \text { patients who are } \\
\text { eligible for OAC, NOACs are recommended in } \\
\text { preference to VKAs in patients with aortic } \\
\text { stenosis, aortic and mitral regurgitation. }\end{array}$ & $\mathbf{I}$ \\
\hline \multicolumn{5}{|c|}{ Section 4. Recommendations on indications for surgery in severe aortic regurgitation } \\
\hline \multirow[t]{2}{*}{ Revised } & $\begin{array}{l}\text { Surgery is indicated in asymptomatic patients } \\
\text { with resting ejection fraction } \leq 50 \% \text {. }\end{array}$ & $\mathbf{I}$ & \multirow{2}{*}{$\begin{array}{l}\text { Surgery is recommended in asymptomatic } \\
\text { patients with LVESD }>50 \mathrm{~mm} \text { or LVESD } \\
>25 \mathrm{~mm} / \mathrm{m}^{2} \text { BSA (in patients with small body } \\
\text { size) or resting LVEF } \leq 50 \% \text {. }\end{array}$} & \multirow[b]{2}{*}{$\mathbf{I}$} \\
\hline & $\begin{array}{l}\text { Surgery should be considered in asymptomatic } \\
\text { patients with resting ejection fraction }>50 \% \\
\text { with severe LV dilatation: LVEDD }>70 \mathrm{~mm} \text { or } \\
\text { LVESD }>50 \mathrm{~mm} \text { (or LVESD }>25 \mathrm{~mm} / \mathrm{m}^{2} \text { BSA in } \\
\text { patients with small body size). }\end{array}$ & Ila & & \\
\hline New & & & $\begin{array}{l}\text { Surgery may be considered in asymptomatic } \\
\text { patients with LVESD }>20 \mathrm{~mm} / \mathrm{m}^{2} \text { BSA } \\
\text { (especially in patients with small body size) or } \\
\text { resting LVEF } \leq 55 \% \text {, if surgery at low-risk. }\end{array}$ & Ilb \\
\hline Revised & $\begin{array}{l}\text { Heart Team discussion is recommended in } \\
\text { selected patients in whom aortic valve repair may } \\
\text { be a feasible alternative to valve replacement. }\end{array}$ & $\mathbf{I}$ & $\begin{array}{l}\text { Aortic valve repair may be considered in } \\
\text { selected patients at experienced centres when } \\
\text { durable results are expected. }\end{array}$ & Illb \\
\hline \multicolumn{4}{|c|}{$\begin{array}{l}\text { Section } 4 \text {. Recommendations on indications for surgery in aortic root or tubular ascending aortic aneurysm (irrespective of the } \\
\text { severity of aortic regurgitation) }\end{array}$} & \\
\hline Revised & $\begin{array}{l}\text { Aortic valve repair, using the reimplantation or } \\
\text { remodelling with aortic annuloplasty technique, } \\
\text { is recommended in young patients with aortic } \\
\text { root dilation and tricuspid aortic valves, when } \\
\text { performed by experienced surgeons. }\end{array}$ & $\mathbf{I}$ & $\begin{array}{l}\text { Valve-sparing aortic root replacement is } \\
\text { recommended in young patients with aortic } \\
\text { root dilation, if performed in experienced } \\
\text { centres and durable results are expected. }\end{array}$ & I \\
\hline \multicolumn{5}{|c|}{ Section 5. Recommendations on indications for intervention in symptomatic and asymptomatic aortic stenosis } \\
\hline \multicolumn{5}{|c|}{ Symptomatic aortic stenosis } \\
\hline Revised & $\begin{array}{l}\text { Intervention is indicated in symptomatic } \\
\text { patients with severe, high-gradient aortic } \\
\text { stenosis (mean gradient } \geq 40 \mathrm{mmHg} \text { or peak } \\
\text { velocity } \geq 4.0 \mathrm{~m} / \mathrm{s} \text { ). }\end{array}$ & I & $\begin{array}{l}\text { Intervention is recommended in symptomatic } \\
\text { patients with severe, high-gradient aortic } \\
\text { stenosis [mean gradient } \geq 40 \mathrm{mmHg} \text {, peak } \\
\text { velocity } \geq 4.0 \mathrm{~m} / \mathrm{s} \text { and valve area } \leq 1.0 \mathrm{~cm}^{2} \text { (or } \\
\leq 0.6 \mathrm{~cm}^{2} / \mathrm{m}^{2} \text { )]. }\end{array}$ & I \\
\hline \multicolumn{5}{|c|}{ Asymptomatic patients with severe aortic stenosis } \\
\hline New & & & $\begin{array}{l}\text { Intervention should be considered in } \\
\text { asymptomatic patients with severe aortic } \\
\text { stenosis and systolic LV dysfunction (LVEF } \\
<55 \% \text { ) without another cause. }\end{array}$ & Ila \\
\hline Revised & $\begin{array}{l}\text { SAVR should be considered in asymptomatic } \\
\text { patients with normal ejection fraction and none } \\
\text { of the above-mentioned exercise test } \\
\text { abnormalities if the surgical risk is low and one } \\
\text { of the following findings is present: } \\
\text { - Very severe aortic stenosis defined by a } \mathrm{V}_{\max } \\
>5.5 \mathrm{~m} / \mathrm{s} \text {. } \\
\text { - Severe valve calcification and a rate of } \mathrm{V}_{\max } \\
\text { progression } \geq 0.3 \mathrm{~m} / \mathrm{s} / \text { year. } \\
\text { - Markedly elevated BNP levels ( }>3 \mathrm{x} \text { age- and } \\
\text { sex-corrected normal range) confirmed by } \\
\text { repeated measurements without other } \\
\text { explanations. } \\
\text { - Severe pulmonary hypertension (systolic } \\
\text { pulmonary artery pressure at rest }>60 \mathrm{mmHg} \\
\text { confirmed by invasive measurement) without } \\
\text { other explanation. }\end{array}$ & Ila & $\begin{array}{l}\text { Intervention should be considered in } \\
\text { asymptomatic patients with } \mathrm{LVEF}>55 \% \text { and } \\
\text { a normal exercise test if the procedural risk is } \\
\text { low and one of the following parameters is } \\
\text { present: } \\
\text { - Very severe aortic stenosis (mean gradient } \\
\geq 60 \mathrm{mmHg} \text { or } \mathrm{V}_{\max } \geq 5 \mathrm{~m} / \mathrm{s} \text { ). } \\
\text { - Severe valve calcification (ideally assessed by } \\
\text { CCT) and } \mathrm{V}_{\max } \text { progression } \geq 0.3 \mathrm{~m} / \mathrm{s} / \mathrm{year} \text {. } \\
\text { - Markedly elevated BNP levels ( }>3 \mathrm{x} \text { age- and } \\
\text { sex-corrected normal range) confirmed by } \\
\text { repeated measurements and without other } \\
\text { explanation. }\end{array}$ & Ila \\
\hline
\end{tabular}


Section 5. Recommended mode of intervention in patients with aortic stenosis

\begin{tabular}{|c|c|}
\hline Revised & $\begin{array}{l}\text { The choice for intervention must be based on } \\
\text { careful individual evaluation of technical } \\
\text { suitability and weighing of risks and benefits of } \\
\text { each modality. In addition, the local expertise } \\
\text { and outcomes data for the given intervention } \\
\text { must be taken into account. }\end{array}$ \\
\hline Revised & $\begin{array}{l}\text { SAVR is recommended in patients at low } \\
\text { surgical risk (STS or EuroSCORE II <4\% or } \\
\text { logistic EuroSCORE I <10\%, and no other risk } \\
\text { factors not included in these scores, such as } \\
\text { frailty, porcelain aorta, sequelae of chest } \\
\text { radiation). }\end{array}$ \\
\hline Revised & $\begin{array}{l}\text { TAVI is recommended in patients who are not } \\
\text { suitable for SAVR as assessed by the Heart } \\
\text { Team. }\end{array}$ \\
\hline Revised & $\begin{array}{l}\text { In patients who are at increased surgical risk } \\
\text { (STS or EuroSCORE II } \geq 4 \% \text { or logistic } \\
\text { EuroSCORE I } \geq 10 \% \text {, or other risk factors not } \\
\text { included in these scores such as frailty, } \\
\text { porcelain aorta, sequelae of chest radiation), } \\
\text { the decision between SAVR and TAVI should be } \\
\text { made by the Heart Team according to the } \\
\text { individual patient characteristics, with TAVI } \\
\text { being favoured in elderly patients suitable for } \\
\text { transfemoral access. }\end{array}$ \\
\hline
\end{tabular}

The choice between surgical and transcatheter intervention must be based upon careful evaluation of clinical, anatomical and procedural factors by the Heart Team, weighing the risks and benefits of each approach for an individual patient. The Heart Team recommendation should be discussed with the patient who can then make an informed treatment choice.

SAVR is recommended in younger patients who are low risk for surgery $(<75$ years and

STS-PROM/ EuroSCORE II $<4 \%$ ) or in patients who are operable and unsuitable for transfemoral TAVI.

TAVI is recommended in older patients

( $\geq 75$ years), or in those who are high-risk (STS-PROM/ EuroSCORE II $>8 \%$ ) or unsuitable for surgery.

SAVR or TAVI are recommended for remaining patients according to individual clinical, anatomical and procedural characteristics.

New

Non-transfemoral TAVI may be considered in patients who are inoperable for SAVR and unsuitable for transfemoral TAVI.

Section 6. Indications for intervention in severe primary mitral regurgitation

\begin{tabular}{|l|l|}
\hline Revised & $\begin{array}{l}\text { Surgery is indicated in asymptomatic patients } \\
\text { with LV dysfunction (LVESD } \geq 45 \mathrm{~mm} \text { and/or } \\
\text { LVEF } \leq 60 \%) .\end{array}$ \\
\hline Revised & $\begin{array}{l}\text { Surgery should be considered in asymptomatic } \\
\text { patients with preserved LV function (LVESD } \\
<45 \mathrm{~mm} \text { and LVEF }>60 \% \text { ) and AF secondary } \\
\text { to mitral regurgitation or pulmonary } \\
\text { hypertension (SPAP at rest }>50 \mathrm{mmHg} \text { ). }\end{array}$ \\
\hline Revised & $\begin{array}{l}\text { Surgery should be considered in asymptomatic } \\
\text { patients with preserved LVEF ( }>60 \% \text { ) and } \\
\text { LVESD 40-44 mm when a durable repair is } \\
\text { likely, surgical risk is low, the repair is } \\
\text { performed in a Heart Valve Centre and at least } \\
\text { one of the following findings is present: } \\
\bullet \text { flail leaflet or; } \\
\text { presence of significant LA dilatation (volume } \\
\text { index } \geq 60 \mathrm{~mL} / \mathrm{m}^{2} \mathrm{BSA} \text { ) in sinus rhythm. }\end{array}$ \\
\hline
\end{tabular}

Surgery is recommended in asymptomatic patients with LV dysfunction (LVESD $\geq 40 \mathrm{~mm}$ and/or LVEF $\leq 60 \%$ ).

Surgery should be considered in asymptomatic patients with preserved LV function (LVESD $<40 \mathrm{~mm}$ and LVEF $>60 \%$ ) and AF secondary to mitral regurgitation or pulmonary hypertension (SPAP at rest $>50 \mathrm{mmHg}$ ).

Surgical mitral valve repair should be considered in low-risk asymptomatic patients with LVEF $>60 \%$, LVESD $<40 \mathrm{~mm}$ and significant $L A$ dilatation (volume index $\geq 60 \mathrm{~mL} / \mathrm{m}^{2}$ or diameter $\geq 55 \mathrm{~mm}$ ) when performed in a Heart Valve Centre and a durable repair is likely.

Section 6. Indications for mitral valve intervention in chronic severe secondary mitral regurgitation

\begin{tabular}{|l|l|l|}
\hline New & & \\
\hline Patients with concomitant coronary artery or other cardiac disease requiring treatim & & \\
\hline New & & \\
\hline Revised & $\begin{array}{l}\text { Surgery is indicated in patients with severe } \\
\text { SMR undergoing CABG and LVEF }>30 \% .\end{array}$ \\
\hline
\end{tabular}

Valve surgery/intervention is recommended only in patients with severe SMR who remain symptomatic despite GDMT (including CRT if indicated) and has to be decided by a structured collaborative Heart Team.

\section{ereatment}

In symptomatic patients, who are judged not appropriate for surgery by the Heart Team on the basis of their individual characteristics, PCl (and/or TAVI) possibly followed by TEER (in case of persisting severe SMR) should be considered.

Valve surgery is recommended in patients undergoing $C A B G$ or other cardiac surgery. 


\section{Patients without concomitant coronary artery or other cardiac disease requiring treatment}

When revascularization is not indicated and surgical risk is not low, a percutaneous edge-to-edge procedure may be considered in patients with severe secondary mitral regurgitation and LVEF $>30 \%$ who remain symptomatic despite optimal medical management (including CRT if indicated) and who have a suitable valve morphology by echocardiography, avoiding futility.

\begin{tabular}{l|l} 
Revised & In patients with severe SMR and LVEF $<30 \%$
\end{tabular} who remain symptomatic despite optimal medical management (including CRT if indicated) and who have no option for revascularization, the Heart Team may consider a percutaneous edge-to-edge procedure or valve surgery after careful evaluation for a ventricular assist device or heart transplant according to individual patient characteristics.

Section 8. Indications for intervention in primary tricuspid regurgitation

Surgery should be considered in asymptomatic or mildly symptomatic patients with severe isolated primary tricuspid regurgitation and progressive RV dilatation or deterioration of RV function.

\begin{tabular}{l|l} 
Revised & After previous left-sided surgery and in absence
\end{tabular} of recurrent left-sided valve dysfunction, surgery should be considered in patients with severe tricuspid regurgitation who are symptomatic or have progressive RV dilatation/ dysfunction, in the absence of severe RV or LV dysfunction and severe pulmonary vascular disease/hypertension.

\begin{tabular}{|l|l|}
\hline New & \\
&
\end{tabular}

Section 11. Recommendations for prosthetic valve selection

\begin{tabular}{|l|l|l|l}
\hline New & & $\begin{array}{l}\text { A bioprosthesis may be considered in patients } \\
\text { already on long-term NOACs due to the high } \\
\text { risk for thromboembolism. }\end{array}$ \\
\hline Revised & $\begin{array}{l}\text { A bioprosthesis should be considered in those } \\
\text { (patients) whose life expectancy is lower than } \\
\text { the presumed durability of the bioprosthesis. }\end{array}$ & $\begin{array}{l}\text { A bioprosthesis is recommended when } \\
\text { good-quality anticoagulation is unlikely } \\
\text { (adherence problems, not readily available), } \\
\text { contraindicated because of high bleeding risk } \\
\text { (previous major bleed, comorbidities, } \\
\text { unwillingness, adherence problems, life-style, } \\
\text { occupation) and in those patients whose life } \\
\text { expectancy is lower than the presumed } \\
\text { durability of the bioprosthesis. }\end{array}$ \\
\hline
\end{tabular}

Section 11. Recommendations for perioperative and postoperative antithrombotic management of valve replacement or repair Management of antithrombotic therapy in the perioperative period

\begin{tabular}{|l|l|l|l|}
\hline New & & $\begin{array}{l}\text { Bridging of OAC, when interruption is needed, } \\
\text { is recommended in patients with any of the } \\
\text { following indication: } \\
\bullet \text { Mechanical prosthetic heart valve. } \\
\text { - AF with significant mitral stenosis. } \\
- \text { AF with a CHA } \mathrm{AS}_{2} \text {-VASc score } \geq 3 \text { for women } \\
\text { or } 2 \text { for men. } \\
\text { - Acute thrombotic event within the previous } \\
4 \text { weeks. } \\
\text { - High acute thromboembolic risk. }\end{array}$ \\
\hline New & $\begin{array}{l}\text { It is recommended that VKAs are timely } \\
\text { discontinued prior to elective surgery to aim for } \\
\text { an INR <1.5. }\end{array}$ \\
\hline
\end{tabular}

Ila

Ila

Transcatheter treatment of symptomatic secondary severe tricuspid regurgitation may be considered in inoperable patients at a Heart Valve Centre with expertise in the treatment of tricuspid valve disease. severe primary tricuspid regurgitation and RV

Surgery should be considered in patients with severe secondary tricuspid regurgitation (with or without previous left-sided surgery) who are symptomatic or have RV dilatation, in the absence of severe RV or LV dysfunction and severe pulmonary vascular disease/ hypertension.

TEER should be considered in selected 作 and fulfilling criteria suggesting an increased

In high-risk symptomatic patients not eligible sugesting an increased chance of responding selected, the Heart Team may consider in trans-catheter valve therapy if applicable, after careful evaluation for ventricular assist device or heart transplant.

Surgery should be considered in asymptomatic or mildly symptomatic patients with isolated

la an INR $<1.5$ 


\begin{tabular}{|c|c|c|c|c|}
\hline New & & & $\begin{array}{l}\text { In patients undergoing surgery, it is } \\
\text { recommended that aspirin therapy, if } \\
\text { indicated, is maintained during the } \\
\text { periprocedural period. }\end{array}$ & I \\
\hline New & & & $\begin{array}{l}\text { In patients who have undergone valve surgery } \\
\text { with an indication for postoperative therapeutic } \\
\text { bridging, it is recommended to start either } \\
\text { UFH or LMWH } 12-24 \text { hours after surgery. }\end{array}$ & $\mathbf{I}$ \\
\hline New & & & $\begin{array}{l}\text { In patients with MHVs, it is recommended to } \\
\text { (re)-initiate VKAs on the first postoperative day. }\end{array}$ & $\mathbf{I}$ \\
\hline New & & & $\begin{array}{l}\text { In patients treated with DAPT after recent } \mathrm{PCl} \\
\text { (within } 1 \text { month) who need to undergo heart } \\
\text { valve surgery, in the absence of an indication } \\
\text { for } \mathrm{OAC} \text {, it is recommended to resume the } \\
\mathrm{P}_{2 \mathrm{Y}} \text { inhibitor postoperatively, as soon as there } \\
\text { is no concern over bleeding. }\end{array}$ & $\mathbf{I}$ \\
\hline New & & & $\begin{array}{l}\text { In patients treated with DAPT after recent PCI } \\
\text { (within } 1 \text { month) who need to undergo heart } \\
\text { valve surgery, in the absence of an indication } \\
\text { for OAC, bridging P2Y } \text { inhibitors with }_{12} \\
\text { glycoprotein IIb/IIla inhibitors or cangrelor may } \\
\text { be considered. }\end{array}$ & Ilb \\
\hline \multicolumn{5}{|c|}{ Patients with an indication to concomitant antiplatelet therapy } \\
\hline Revised & $\begin{array}{l}\text { In patients undergoing an uncomplicated PCI } \\
\text { dual therapy comprising VKA and clopidogrel } \\
\text { (75 mg/day) should be considered as an } \\
\text { alternative to 1-month triple antithrombotic } \\
\text { therapy in patients in whom the bleeding risk } \\
\text { outweighs the ischaemic risk. }\end{array}$ & Ila & $\begin{array}{l}\text { After uncomplicated } \mathrm{PCI} \text { or ACS in patients } \\
\text { requiring long -term OAC, early cessation ( } \leq 1 \\
\text { week) of aspirin and continuation of dual } \\
\text { therapy with } \mathrm{OAC} \text { and a } \mathrm{P} 2 \mathrm{Y}_{12} \text { inhibitor } \\
\text { (preferably clopidogrel) for up to } 6 \text { months (or } \\
\text { up to } 12 \text { months in ACS) is recommended if } \\
\text { the risk of stent thrombosis is low or if } \\
\text { concerns about bleeding risk prevail over } \\
\text { concerns about risk of stent thrombosis, } \\
\text { irrespective of the type of stent used. }\end{array}$ & $\mathbf{I}$ \\
\hline New & & & $\begin{array}{l}\text { Discontinuation of antiplatelet treatment in } \\
\text { patients treated with an OAC is recommended } \\
\text { after } 12 \text { months. }\end{array}$ & I \\
\hline New & & & $\begin{array}{l}\text { In patients treated with a VKA (e.g. MHVs), } \\
\text { clopidogrel alone should be considered in } \\
\text { selected patients (e.g. HAS-BLED } \geq 3 \text { or } \\
\text { ARC-HBR met and low risk of stent } \\
\text { thrombosis) for up to } 12 \text { months. }\end{array}$ & Ila \\
\hline New & & & $\begin{array}{l}\text { In patients requiring aspirin and/or clopidogrel } \\
\text { in addition to VKA, the dose intensity of VKA } \\
\text { should be considered and carefully regulated } \\
\text { with a target INR in the lower part of the } \\
\text { recommended target range and a time in the } \\
\text { therapeutic range }>65-70 \% \text {. }\end{array}$ & Ila \\
\hline New & & & $\begin{array}{l}\text { After uncomplicated } \mathrm{PCI} \text { or ACS in patients } \\
\text { requiring both OAC and antiplatelet therapy, } \\
\text { triple therapy with aspirin, clopidogrel and OAC } \\
\text { for longer than } 1 \text { week should be considered } \\
\text { when the risk of stent thrombosis outweighs } \\
\text { the risk of bleeding, with a total duration } \\
\text { ( } \leq 1 \text { month) decided according to assessment } \\
\text { of these risks and clearly specified at hospital } \\
\text { discharge. }\end{array}$ & Ila \\
\hline \multicolumn{5}{|c|}{ Surgical valve replacement } \\
\hline New & & & $\begin{array}{l}\text { NOACs should be considered over VKA after } \\
3 \text { months following surgical implantation of } \\
\text { a BHV, in patients with AF. }\end{array}$ & Ila \\
\hline New & & & $\begin{array}{l}\text { In patients with no baseline indications for } \\
\text { OAC, low-dose aspirin ( } 75-100 \mathrm{mg} / \text { day) or OAC } \\
\text { using a VKA should be considered for the first } \\
3 \text { months after surgical implantation of an } \\
\text { aortic BHV. }\end{array}$ & Ila \\
\hline New & & & $\begin{array}{l}\text { NOACs may be considered over VKA within } \\
3 \text { months following surgical implantation of } \\
\text { a BHV in mitral position in patients with AF. }\end{array}$ & Illb \\
\hline
\end{tabular}


Transcatheter Aortic Valve Implantation

\begin{tabular}{|c|c|c|c|c|}
\hline New & & & $\begin{array}{l}\text { OAC is recommended lifelong for TAVI patients } \\
\text { who have other indications for OAC. }\end{array}$ & I \\
\hline Revised & $\begin{array}{l}\text { SAPT may be considered after TAVI in the case } \\
\text { of high bleeding risk. }\end{array}$ & Ilb & $\begin{array}{l}\text { Lifelong SAPT is recommended after TAVI in } \\
\text { patients with no baseline indication for OAC. }\end{array}$ & I \\
\hline New & & & $\begin{array}{l}\text { Routine use of OAC is not recommended after } \\
\text { TAVI in patients with no baseline indication for } \\
\text { OAC. }\end{array}$ & III \\
\hline \multicolumn{5}{|c|}{ Section 11. Recommendations on management of prosthetic valve dysfunction } \\
\hline \multicolumn{5}{|c|}{ Haemolysis and paravalvular leak } \\
\hline New & & & $\begin{array}{l}\text { Decision on transcatheter or surgical closure of } \\
\text { clinically significant paravalvular leaks should } \\
\text { be considered based on patient risk status, } \\
\text { leak morphology, and local expertise. }\end{array}$ & Ila \\
\hline \multicolumn{5}{|c|}{ Bioprosthetic thrombosis } \\
\hline New & & & $\begin{array}{l}\text { Anticoagulation should be considered in } \\
\text { patients with leaflet thickening and reduced } \\
\text { leaflet motion leading to elevated gradients, at } \\
\text { least until resolution. }\end{array}$ & Ila \\
\hline \multicolumn{5}{|c|}{ Bioprosthetic failure } \\
\hline New & & & $\begin{array}{l}\text { Transcatheter valve-in-valve implantation in the } \\
\text { mitral and tricuspid position may be } \\
\text { considered in selected patients at high-risk for } \\
\text { surgical re-intervention. }\end{array}$ & Illb \\
\hline \multicolumn{5}{|c|}{$\begin{array}{l}\text { ACS: acute coronary syndrome; AF: atrial fibrillation; ARC-HBR: Academic Research Consortium - high bleeding risk; BHV: biological heart valve; } \\
\text { BNP: B-type natriuretic peptide; BSA: body surface area; CABG: Coronary artery bypass grafting; CCT: cardiac computed tomography; CRT: cardiac } \\
\text { resynchronization therapy; DAPT: dual anti-platelet therapy; EuroSCORE: European System for Cardiac Operative Risk Evaluation; GDMT: guideline- } \\
\text { directed medical therapy; INR: international normalized ratio; LA: left atrium/left atrial; LAA: left atrial appendage; LMWH: low-molecular-weight } \\
\text { heparin; LV: left ventricle/left ventricular; LVEDD: left ventricular end-diastolic diameter; LVEF: left ventricular ejection fraction; LVESD: Left ventricular } \\
\text { end-systolic diameter; MHV: mechanical heart valve; NOAC: non-vitamin K antagonist oral anticoagulant; OAC: oral anticoagulation; PCI: percutaneous } \\
\text { coronary intervention; RV: right ventricle/right ventricular; SAPT: single antiplatelet therapy; SAVR: surgical aortic valve replacement; SMR: secondary } \\
\text { mitral regurgitation; SPAP: systolic pulmonary arterial pressure; STS-PROM: Society of Thoracic Surgeons - predicted risk of mortality; } \\
\text { TAVI: transcatheter aortic valve implantation; TEER: transcatheter edge-to-edge repair; UFH: unfractionated heparin; VHD: valvular heart disease; } \\
\text { VKA: vitamin K antagonist; } V_{\text {max }} \text { : peak transvalvular velocity. }\end{array}$} \\
\hline
\end{tabular}

recommendations. The group was constituted of two European Society of Cardiology (ESC) and two European Association for Cardio-Thoracic Surgery (EACTS) delegates who were also members of the Task Force. Although the principle activities of the group concerned the chapter on aortic stenosis and SMR, it was not limited to these two domains. The methodology group was at disposal, upon request of the Task Force members, to resolve specific methodological issues.

\subsection{CONTENT OF THESE GUIDELINES}

Decision making in VHD involves accurate diagnosis, timing of intervention, risk assessment and, based on these, selection of the most suitable type of intervention. These guidelines focus on acquired VHD, are oriented towards management, and do not deal with endocarditis ${ }^{4}$ congenital valve disease ${ }^{5}$ (including pulmonary valve disease), or recommendations concerning sports cardiology and exercise in patients with cardiovascular disease, ${ }^{6}$ as separate guidelines have been published by the ESC on these topics.

\subsection{NEW FORMAT OF THE GUIDELINES}

The new guidelines have been adapted to facilitate their use in clinical practice and to meet readers' demands by focusing on condensed, clearly represented recommendations. At the end of the document, key points summarize the essentials. Gaps in evidence are listed to propose topics for future research. The guideline document will be harmonized with the chapter on VHD included in the ESC Textbook of Cardiovascular Medicine (ISBN: 9780198784906). The guidelines and the textbook are complementary. Background information and detailed discussion of the data that have provided the basis for the recommendations will be found in the relevant book chapter.

\subsection{HOW TO USE THESE GUIDELINES}

The Committee emphasizes that many factors ultimately determine the most appropriate treatment in individual patients within a given community. These factors include the availability of diagnostic equipment, the expertise of cardiologists and surgeons, especially in the field of valve repair and percutaneous intervention, and, notably, the wishes of well-informed patients. Furthermore, owing to the lack of evidence-based data in the field of VHD, most recommendations are largely the result of expert consensus opinion. Therefore, deviations from these guidelines may be appropriate in certain clinical circumstances.

\section{General comments}

This section defines and discusses concepts common to all the types of VHD including the Heart Team and Heart Valve Centres, 
the main evaluation steps of patients presenting with VHD, as well as the most commonly associated cardiac diseases.

\subsection{CONCEPTS OF HEART TEAM AND HEART VALVE CENTRE}

The main purpose of Heart Valve Centres as centres of excellence in the treatment of VHD is to deliver optimal quality of care with a patient-centred approach. The main requirements of a Heart Valve Centre are presented in Table 4.

This is achieved through high procedural volume in conjunction with specialized training, continuous education, and focused clinical interest. Heart Valve Centres should promote timely referral of patients with VHD for comprehensive evaluation before irreversible damage occurs.

Decisions concerning treatment and intervention should be made by an active and collaborative Heart Team with expertise in VHD, comprising clinical and interventional cardiologists, cardiac surgeons, imaging specialists with expertise in interventional imaging, ${ }^{7,8}$ cardiovascular anaesthesiologists, and other specialists if necessary (e.g. heart failure specialists or electrophysiologists). Dedicated nursing personnel with expertise in the care of patients with VHD are also an important asset to the Heart Team. The Heart Team approach is particularly advisable for the management of high-risk and asymptomatic patients, as well as in case of uncertainty or lack of strong evidence.

Heart Valve Clinics are an important component of the Heart Valve Centres, aiming to provide standardized organization of care based on guidelines. Access to Heart Valve Clinics improves outcomes.?

Table 4. Requirements for a Heart Valve Centre.

\section{Requirements}

Centre performing heart valve procedures with institutional cardiology and cardiac surgery departments with 24 h/7-day services.

Heart Team: clinical cardiologist, interventional cardiologist, cardiac surgeon, imaging specialist with expertise in interventional imaging, cardiovascular anaesthesiologist.

Additional specialists if required: heart failure specialist, electrophysiologist, geriatrician and other specialists (intensive care, vascular surgery, infectious disease, neurology). Dedicated nursing personnel is an important asset to the Heart Team.

The Heart Team must meet on a frequent basis and work with standard operating procedures and clinical governance arrangements defined locally.

A hybrid catheterization laboratory is desirable.

The entire spectrum of surgical and transcatheter valve procedures should be available.

High volume for hospital and individual operators.

Multimodality imaging including echocardiography, CCT, CMR, and nuclear medicine, as well as expertise on guidance of surgical and interventional procedures.

Heart Valve Clinic for outpatient and follow-up management. Data review: continuous evaluation of outcomes with quality review and/or local/external audit.

Education programmes targeting patient primary care, operator, diagnostic and interventional imager training and referring cardiologist.

CCT: cardiac computed tomography; CMR: cardiac magnetic resonance.
Physicians experienced in the management of VHD and dedicated nurses organize outpatient visits, and referral to the Heart Team, if needed. Earlier referral should be encouraged if patient's symptoms develop or worsen before the next planned visit. ${ }^{10,11}$

Beside the whole spectrum of valvular interventions, expertise in interventional and surgical management of coronary artery disease (CAD), vascular diseases, and complications must be available.

Techniques with a steep learning curve may be performed with better results at hospitals with high procedural volume and experience. The relationship between case volume and outcomes for surgery and transcatheter interventions is complex but should not be denied. ${ }^{12-14}$ However, the precise numbers of procedures per individual operator or hospital required to provide high-quality care remain controversial as inequalities exist between high- and middle-income countries. ${ }^{15}$ High-volume TAVI programmes are associated with lower mortality at 30 days, particularly at hospitals with a high surgical aortic valve replacement (SAVR) volume. ${ }^{16,17}$ The data available on transcatheter mitral valve repair ${ }^{14,18}$ and, even more so, transcatheter tricuspid procedures are more limited.

Since performance does not exclusively relate to intervention volume, internal quality assessment consisting of systematic recording of procedural data and patient outcomes at the level of a given Heart Valve Centre is essential, as well as participation in national or ESC/EACTS registries.

A Heart Valve Centre should have structured and possibly combined training programmes for interventionalists, cardiac surgeons, and imaging specialists ${ }^{13,19,20}$ (https://ebcts.org/syllabus/). New techniques should be taught by competent mentors to minimize the effects of the learning curve.

Finally, Heart Valve Centres should contribute to optimizing the management of patients with VHD, provide corresponding services at the community level, and promote networks that include other medical departments, referring cardiologists and primary care physicians.

\subsection{PATIENT EVALUATION}

The aims of the evaluation of patients with VHD are to diagnose, quantify, and assess the mechanism of VHD, as well as its consequences.

\subsubsection{CLINICAL EVALUATION}

Precise evaluation of the patient's history and symptomatic status, and proper physical examination, in particular auscultation ${ }^{21}$ and search for heart failure signs, are crucial. In addition, assessment of their comorbidities and general condition require particular attention. The essential questions in the evaluation of a patient for valvular intervention are summarized in Figure 1 (Central illustration).

\subsubsection{ECHOCARDIOGRAPHY}

Following adequate clinical evaluation, echocardiography is the key technique used to confirm the diagnosis of VHD, as well as to assess its aetiology, mechanisms, function, severity, and prognosis. It should be performed and interpreted by properly trained imagers. 22,23 


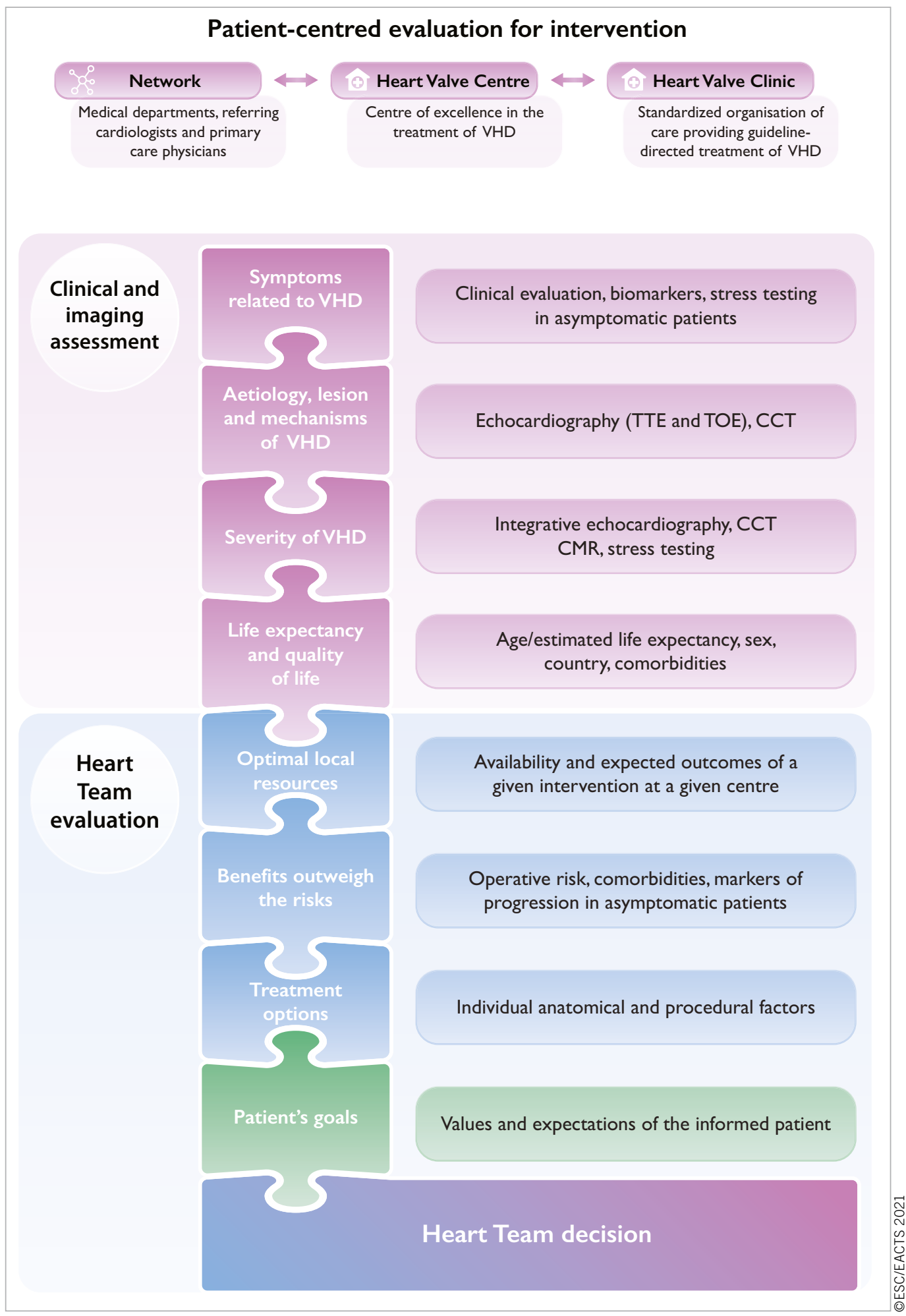

Figure 1. Central illustration: Patient-centred evaluation for intervention. VHD: valvular heart disease; CCT: cardiac computed tomography; CMR: cardiac magnetic resonance; TOE: transoesophageal echocardiography; TTE: transthoracic echocardiography.

Echocardiographic criteria for the definition of severe valve stenosis and regurgitation are addressed in specific documents ${ }^{24,25}$ and summarized in the specific sections of these guidelines. Echocardiography is also key to evaluating the feasibility of a specific intervention.
Indices of left ventricular (LV) enlargement and function are strong prognostic factors. Recent studies suggest that global longitudinal strain has greater prognostic value than LV ejection fraction (LVEF), although cut-off values are not uniform. ${ }^{26,27}$ Transoesophageal echocardiography (TOE) should be considered 
when transthoracic echocardiography (TTE) is of suboptimal quality or when thrombosis, prosthetic valve dysfunction, or endocarditis is suspected. TOE is useful when detailed functional valve anatomy is required to assess repairability. Intraprocedural TOE, preferably $3 \mathrm{D}$, is used to guide transcatheter mitral and tricuspid valve procedures and to assess the immediate result of surgical valve operations. Multimodality imaging may be required in specific conditions for evaluation and/or procedural guidance in TAVI and transcatheter mitral interventions. ${ }^{28,29}$

\subsubsection{OTHER NON-INVASIVE INVESTIGATIONS}

\subsubsection{Stress testing}

The primary purpose of exercise testing is to unmask the objective occurrence of symptoms in patients who claim to be asymptomatic. It is especially useful for risk stratification in aortic stenosis. ${ }^{30}$ Exercise testing will also determine the level of recommended physical activity, including participation in sports. It should be emphasized that stress testing is safe and useful in asymptomatic patients with VHD. Unfortunately, the VHD II survey indicates that it is rarely performed in asymptomatic patients. ${ }^{1}$

Exercise echocardiography may identify the cardiac origin of dyspnoea. Prognostic impact has been shown mainly for aortic stenosis and mitral regurgitation..$^{31,32}$

The use of stress tests to detect CAD associated with severe valvular disease is discouraged because of their low diagnostic value and potential risks in symptomatic patients with aortic stenosis.

\subsubsection{Cardiac magnetic resonance}

In patients with inadequate echocardiographic quality or discrepant results, CMR should be used to assess the severity of valvular lesions, particularly regurgitant lesions, and to assess ventricular volumes, systolic function, abnormalities of the ascending aorta, and myocardial fibrosis. ${ }^{33} \mathrm{CMR}$ is the reference method for the evaluation of right ventricular (RV) volumes and function and is therefore particularly useful to evaluate the consequences of tricuspid regurgitation. ${ }^{34}$ It also has an incremental value for assessing the severity of aortic and mitral regurgitation.

\subsubsection{Computed tomography}

CCT may contribute to the evaluation of valve disease severity, particularly in aortic stenosis ${ }^{35,36}$ and possibly associated disease of the thoracic aorta (dilatation, calcification), as well as to evaluate the extent of MAC. CCT should be performed whenever the echocardiographic data indicate an aortic enlargement $>40 \mathrm{~mm}$, to clarify aortic diameter and to assess aortic morphology and configuration. CCT is essential in the pre-procedural planning of TAVI and can also be useful to assess patient-prosthesis mismatch (PPM) ${ }^{37}$ It is also a prerequisite for pre-procedural planning of mitral and tricuspid valve interventions. ${ }^{38}$ Positron emission tomography (PET)/CCT is useful in patients with a suspicion of endocarditis of a prosthetic valve..$^{39,40}$ 3.2.3.4 Cinefluoroscopy

Cinefluoroscopy is particularly useful for assessing the kinetics of the leaflet occluders of a mechanical prosthesis.

\subsubsection{Biomarkers}

B-type natriuretic peptide (BNP) serum levels, corrected for age and sex, are useful in asymptomatic patients and may assist selection of the appropriate time point for a given intervention, ${ }^{41}$ particularly if the level rises during follow-up. Other biomarkers have been tested, with evidence for fibrosis, inflammation, and adverse ventricular remodelling, which could improve decision making. ${ }^{42}$

\subsubsection{Multimarkers and staging}

In patients with at least moderate aortic stenosis and LVEF $>50 \%$, staging according to damage associated with aortic stenosis on LV/ $\mathrm{RV}$, left atrium (LA), mitral /tricuspid valve, and pulmonary circulation was predictive of excess mortality after TAVI and SAVR, and may help to identify patients who will benefit from an intervention..$^{43,44}$ 3.2.4 INVASIVE INVESTIGATIONS

\subsubsection{Coronary angiography}

Coronary angiography is recommended for the assessment of CAD when surgery or an intervention is planned, to determine if concomitant coronary revascularization is recommended (see recommendations for management of CAD in patients with VHD). ${ }^{45,46}$ Alternatively, owing to its high negative predictive value, CCT may be used to rule out CAD in patients who are at low risk of atherosclerosis. The usefulness of fractional flow reserve or instantaneous wave-free ratio in patients with VHD is not well established, and caution is warranted in the interpretation of these measurements when VHD, and in particular aortic stenosis, is present. ${ }^{47,48}$

\subsubsection{Cardiac catheterization}

The measurement of pressures and cardiac output or the assessment of ventricular performance and valvular regurgitation by ventricular angiography or aortography is restricted to situations where non-invasive evaluation by multimodality imaging is inconclusive or discordant with clinical findings. When elevated, pulmonary pressure is the only criterion to support the indication for surgery, and confirmation of echo data by invasive measurement is recommended. Right heart catheterization is also indicated in patients with severe tricuspid regurgitation as Doppler gradient may be impossible or underestimate the severity of pulmonary hypertension.

\subsubsection{ASSESSMENT OF COMORBIDITY}

The choice of specific examinations to assess comorbidity is guided by the clinical evaluation.

\subsection{RISK STRATIFICATION}

Risk stratification applies to any sort of intervention and is required for weighing the risk of intervention against the expected natural history of VHD and for choosing the type of intervention. Most experience relates to surgery and TAVI.

\subsubsection{RISK SCORES}

The Society of Thoracic Surgeons (STS) predicted risk of mortality (PROM) score (http://riskcalc.sts.org/stswebriskcalc/calculate) and the European System for Cardiac Operative Risk Evaluation II (EuroSCORE II; http://www.euroscore.org/calc.html) accurately discriminate high- and low-risk surgical patients and show good calibration to predict postoperative outcome after valvular surgery in the majority of the patients, ${ }^{49,50}$ while risk estimation may be less accurate in high-risk patients. ${ }^{51}$ The STS-PROM score is dynamic and changes over time. Of note, the risk scores have not been validated for isolated tricuspid surgical interventions. 
In isolation, surgical scores have major limitations for practical use in patients undergoing transcatheter intervention because they do not include major risk factors such as frailty, as well as anatomical factors with impact on the procedure, either surgical or transcatheter [porcelain aorta, previous chest radiation, mitral annular calcification (MAC)].

New scores have been developed to estimate the risk in patients undergoing TAVI, with better accuracy and discrimination than the surgical risk scores, despite numerous limitations ${ }^{52-54}$ (Supplementary Table 1).

Experience with risk stratification is currently limited for other interventional procedures, such as mitral or tricuspid interventions.

\subsubsection{OTHER FACTORS}

Other factors should be taken into account:

- Frailty, defined as a decrease of physiologic reserve and ability to maintain homeostasis leading to an increased vulnerability to stresses and conferring an increased risk of morbidity and mortality after both surgery and TAVI. ${ }^{55}$ The assessment of frailty should not rely on a subjective approach, such as the 'eyeball test', but rather on a combination of different objective estimates. ${ }^{55-59}$ Several tools are available for assessing frailty (Supplementary Table 2, ${ }^{59}$ and Supplementary Table 3). ${ }^{60}$

- Malnutrition ${ }^{61}$ and cognitive dysfunction ${ }^{62}$ both predict poor prognosis.

- Other major organ failures (Supplementary Table 4), in particular the combination of severe lung disease, ${ }^{63,64}$ postoperative pain from sternotomy or thoracotomy and prolonged time under anaesthesia in patients undergoing SAVR via full sternotomy, may contribute to pulmonary complications. There is a positive association between the impairment of renal function and increased mortality after valvular surgery and transcatheter procedures, ${ }^{65}$ especially when the glomerular filtration rate is $<30 \mathrm{~mL} /$ min. Liver disease, is also an important prognostic factor. ${ }^{66}$

- Anatomical aspects affecting procedural performance such as porcelain aorta or severe $\mathrm{MAC}^{67}$ (see Table 6 in section 5.1.3, and Supplementary Figure 1).

At the extreme of the risk spectrum, futility should be avoided. Therapeutic futility has been defined as a lack of medical efficacy, particularly when the physician judges that the therapy is unlikely to produce its intended clinical results, or lack of meaningful survival according to the personal values of the patient. Assessment of futility goes beyond survival and includes functional recovery. The futility of interventions has to be taken into consideration, particularly for transcatheter interventions. ${ }^{63}$

The high prevalence of comorbidity in the elderly makes assessment of the risk/benefit ratios of interventions more difficult, therefore the role of the Heart Team is essential in this specific population of patients (Supplementary Table 5).

\subsection{PATIENT-RELATED ASPECTS}

Patient-related life expectancy and expected quality of life should be considered. The patient and their family should be thoroughly informed and assisted in their decision on the best treatment option. ${ }^{13}$ A patient-centred approach would take patient-reported outcome measures and patient-reported experience measures into consideration and make these parameters part of the informed choice offered to patients. ${ }^{68,69}$

When benefit in symptom relief aligns with a patient's goals, care is not futile. However, care is futile when no life prolongation or symptom relief is anticipated. ${ }^{70}$

\subsection{LOCAL RESOURCES}

Even if it is desirable that Heart Valve Centres are able to perform a large spectrum of procedures, either surgical or catheter-based, specialization and thereby expertise in specific domains will vary and should be taken into account when deciding on the orientation of the patient in specific cases, such as complex surgical valve repair or transcatheter intervention.

In addition, penetration of transcatheter interventions is heterogeneous worldwide and highly dependent on socioeconomic inequalities. ${ }^{15,71}$ Appropriate stewardship of economic resources is a fundamental responsibility of the Heart Team.

\subsection{MANAGEMENT OF ASSOCIATED CONDITIONS}

\subsubsection{CORONARY ARTERY DISEASE}

Recommendations for the management of CAD associated with VHD are provided below and are detailed in specific sections (section 5 and section 6.2) of this guideline document, as well as in other dedicated guideline documents. ${ }^{45,46,72,73}$

\subsubsection{ATRIAL FIBRILLATION}

Detailed recommendations on the management of patients with atrial fibrillation (AF) including management of anticoagulation are provided in specific guidelines. ${ }^{74}$ NOACs are recommended in patients with aortic stenosis, aortic regurgitation or mitral regurgitation presenting with $\mathrm{AF}^{75-78}$ as subgroup analyses of randomized controlled trials (RCTs) support the use of apixaban, dabigatran, edoxaban, and rivaroxaban. The use of NOACs is not recommended in patients who have AF associated with clinically significant mitral stenosis or those with mechanical prostheses.

Surgical ablation of AF combined with mitral valve surgery effectively reduces the incidence of AF but has no impact on adjusted short-term survival. An increased rate of pacemaker implantation has been observed after surgical ablation $(9.5 \%$, vs. $7.6 \%$ in the group with AF and no surgical ablation). ${ }^{79}$ Concomitant $\mathrm{AF}$ ablation should be considered in patients undergoing cardiac surgery, balancing the benefits of freedom from atrial arrhythmias with the risk factors for recurrence, such as age, LA dilatation, years in AF, renal dysfunction, and other cardiovascular risk factors. In addition, left atrial appendage (LAA) occlusion should be considered in combination with valve surgery in patients with $\mathrm{AF}$ and a $\mathrm{CHA}_{2} \mathrm{DS}_{2}$ VASc score $\geq 2$ to reduce the thromboembolic risk. ${ }^{80-82}$ The selected surgical technique should ensure complete occlusion of the LAA. For patients with AF and risk factors for stroke, long-term oral anticoagulation (OAC) is currently recommended, irrespective of the use of surgical ablation of AF and/or surgical LAA occlusion. 


\begin{tabular}{|c|c|c|}
\hline \multicolumn{3}{|c|}{ Recommendations for management of CAD in patients with VHD } \\
\hline Recommendations & Class $^{\mathrm{a}}$ & Level $^{\mathrm{b}}$ \\
\hline \multicolumn{3}{|l|}{ Diagnosis of CAD } \\
\hline $\begin{array}{l}\text { Coronary angiography is recommended before } \\
\text { valve surgery in patients with severe VHD and any } \\
\text { of the following: } \\
\text { - History of cardiovascular disease. } \\
\text { - Suspected myocardial ischaemia. } \\
\text { - LV systolic dysfunction. } \\
\text { - In men >40 years of age and postmenopausal } \\
\text { women. } \\
\text { - One or more cardiovascular risk factors. }\end{array}$ & I & C \\
\hline $\begin{array}{l}\text { Coronary angiography is recommended in the } \\
\text { evaluation of severe SMR. }\end{array}$ & I & c \\
\hline $\begin{array}{l}\text { Coronary CT angiography should be considered as } \\
\text { an alternative to coronary angiography before } \\
\text { valve surgery in patients with severe VHD and low } \\
\text { probability of CAD. }{ }^{d}\end{array}$ & Ila & C \\
\hline \multicolumn{3}{|l|}{ Indications for myocardial revascularization } \\
\hline $\begin{array}{l}\text { CABG is recommended in patients with a primary } \\
\text { indication for aortic/mitral/tricuspid valve surgery } \\
\text { and coronary artery diameter stenosis } \geq 70 \% \text {., }\end{array}$ & I & C \\
\hline $\begin{array}{l}\text { CABG should be considered in patients with } \\
\text { a primary indication for aortic/mitral/tricuspid } \\
\text { valve surgery and coronary artery diameter } \\
\text { stenosis } \geq 50-70 \% \text {. }\end{array}$ & Ila & C \\
\hline $\begin{array}{l}\mathrm{PCl} \text { should be considered in patients with } \\
\text { a primary indication to undergo TAVI and } \\
\text { coronary artery diameter stenosis }>70 \% \text { in } \\
\text { proximal segments. }\end{array}$ & Ila & C \\
\hline $\begin{array}{l}\mathrm{PCl} \text { should be considered in patients with } \\
\text { a primary indication to undergo transcatheter } \\
\text { mitral valve intervention and coronary artery } \\
\text { diameter stenosis }>70 \% \text { in proximal segments. }\end{array}$ & Ila & C \\
\hline
\end{tabular}

CABG: coronary artery bypass grafting; CAD: coronary artery disease; $\mathrm{CT}$ : computed tomography; LV: left ventricle/left ventricular;

$\mathrm{PCl}$ : percutaneous coronary intervention; SMR: secondary mitral regurgitation; TAVI: transcatheter aortic valve implantation;

VHD: valvular heart disease. ${ }^{a}$ Class of recommendation. ${ }^{\text {b }}$ Level of evidence. ${ }^{c}$ Chest pain, abnormal non-invasive testing. ${ }^{\mathrm{d}}$ Coronary $\mathrm{C}$ angiography may also be used in patients requiring emergency surgery with acute infective endocarditis with large vegetations protruding in front of a coronary ostium. ${ }^{\text {e }}$ Stenosis $\geq 50 \%$ can be considered for left main stenosis. ${ }^{\mathrm{f} F F R} \leq 0.8$ is a useful cut-off indicating the need for an intervention in patients with mitral or tricuspid diseases, but has not been validated in patients with aortic stenosis. Adapted from ${ }^{45,72}$

Recommendations for the management of AF in native VHD are summarized in the following table. The recommendations concerning patients with valve prostheses, and the combination of anticoagulants and antiplatelet agents in patients undergoing PCI, are described in section 11 (section 11.3.2.2 and related table of recommendations for perioperative and postoperative antithrombotic management of valve replacement or repair).

\subsection{ENDOCARDITIS PROPHYLAXIS}

Antibiotic prophylaxis should be considered for high-risk procedures in patients with prosthetic valves, including transcatheter valves, or with repairs using prosthetic material, and in patients with previous episode(s) of infective endocarditis. ${ }^{4}$ Particular attention to dental and cutaneous hygiene and strict aseptic measures during any invasive procedure are advised in this population. Antibiotic prophylaxis should be considered in dental procedures involving manipulation of the gingival or periapical region of the teeth or manipulation of the oral mucosa. ${ }^{4}$

\section{Recommendations on management of atrial fibrillation in patients with native VHD}

\begin{tabular}{|c|c|c|}
\hline Recommendations & Class $^{\mathrm{a}}$ & Level $^{\mathrm{b}}$ \\
\hline \multicolumn{3}{|l|}{ Anticoagulation } \\
\hline $\begin{array}{l}\text { For stroke prevention in AF patients who are } \\
\text { eligible for OAC, NOACs are recommended in } \\
\text { preference to VKAs in patients with aortic } \\
\text { stenosis, aortic and mitral regurgitation. }{ }^{75-78,83,84}\end{array}$ & I & A \\
\hline $\begin{array}{l}\text { The use of NOACs is not recommended in } \\
\text { patients with AF and moderate to severe mitral } \\
\text { stenosis. }\end{array}$ & III & C \\
\hline \multicolumn{3}{|l|}{ Surgical interventions } \\
\hline $\begin{array}{l}\text { Concomitant AF ablation should be considered in } \\
\text { patients undergoing valve surgery, balancing the } \\
\text { benefits of freedom from atrial arrhythmias and } \\
\text { the risk factors for recurrence (LA dilatation, } \\
\text { years in AF, age, renal dysfunction, and other } \\
\text { cardiovascular risk factors). } .^{79,85-90}\end{array}$ & Ila & A \\
\hline $\begin{array}{l}\text { LAA occlusion should be considered to reduce } \\
\text { the thromboembolic risk in patients, with } \mathrm{AF} \text { and } \\
\text { a } \mathrm{CHA}_{2} \mathrm{DS}_{2} \mathrm{VASc} \text { score } \geq 2 \text { undergoing valve } \\
\text { surgery. }\end{array}$ & Ila & B \\
\hline
\end{tabular}

\subsection{PROPHYLAXIS FOR RHEUMATIC FEVER}

Prevention of rheumatic heart disease should preferably target the first attack of acute rheumatic fever. Antibiotic treatment of group A Streptococcus infection throat is key in primary prevention. Echocardiographic screening in combination with secondary antibiotic prophylaxis in children with evidence of latent rheumatic heart disease is currently investigated to reduce its prevalence in endemic regions. ${ }^{91}$ In patients with established rheumatic heart disease, secondary long-term prophylaxis against rheumatic fever is recommended: benzathine benzyl penicillin 1.2 MUI every 3 to 4 weeks over 10 years. Lifelong prophylaxis should be considered in high-risk patients according to the severity of VHD and exposure to group A Streptococcus. ${ }^{92-95}$

\section{Aortic regurgitation}

Aortic regurgitation can be caused by primary disease of the aortic valve cusps and/or abnormalities of the aortic root and ascending aortic geometry. Degenerative tricuspid and bicuspid aortic regurgitation are the most common aetiologies in high-income countries, accounting for approximately two-thirds of the underlying aetiology of aortic regurgitation in the EURObservational Registry Programme Valvular Heart Disease II registry. ${ }^{1}$ Other causes include infective and rheumatic endocarditis. Acute severe aortic regurgitation is mostly caused by infective endocarditis, and less frequently by aortic dissection.

\subsection{EVALUATION}

\subsubsection{ECHOCARDIOGRAPHY}

Echocardiography is the key examination used to describe valve anatomy, quantify aortic regurgitation, evaluate its mechanisms, define the morphology of the aorta, and determine the feasibility 
of valve-sparing aortic surgery or valve repair. ${ }^{96,97}$ Identification of the mechanism follows the same principle such as for mitral regurgitation: normal cusps but insufficient coaptation due to dilatation of the aortic root with central jet (type 1), cusp prolapse with eccentric jet (type 2), or retraction with poor cusp tissue quality and large central or eccentric jet (type 3). ${ }^{96}$ Quantification of aortic regurgitation follows an integrated approach considering qualitative, semi-quantitative, and quantitative parameters ${ }^{24,98}$ (Table 5). New parameters obtained by 3D echocardiography and two-dimensional (2D) strain imaging as LV global longitudinal strain may be useful, particularly in patients with borderline LVEF where they may help in the decision for surgery. ${ }^{99}$ Measurement of the aortic root and ascending aorta in 2D is performed at four levels: annulus, sinuses of Valsalva, sinotubular junction, and tubular ascending aorta. ${ }^{100,101}$ Measurements are performed in the parasternal long-axis view from leading edge to leading edge at end diastole, except for the aortic annulus, which is measured in mid systole. As it will have surgical consequences, it is important to differentiate three phenotypes of the ascending aorta: aortic root aneurysms (sinuses of Valsalva $>45 \mathrm{~mm}$ ), tubular ascending aneurysm (sinuses of Valsalva $<40-45 \mathrm{~mm}$ ), and isolated aortic regurgitation (all aortic diameters $<40 \mathrm{~mm}$ ). The calculation of indexed values to account for body size has been suggested, ${ }^{102}$ in particular in patients with small stature. Anatomy of the aortic valve cusps and its suitability for valve repair should be provided by preoperative TOE if aortic valve repair or a valve-sparing surgery of the aortic root is considered. Intraoperative evaluation of the surgical

\section{Table 5. Echocardiographic criteria for the definition of severe aortic valve regurgitation.}

\begin{tabular}{|c|c|}
\hline \multicolumn{2}{|c|}{ Qualitative } \\
\hline Valve morphology & Abnormal/flail/large coaptation defect \\
\hline $\begin{array}{l}\text { Colour flow regurgitant jet } \\
\text { area }^{a}\end{array}$ & $\begin{array}{l}\text { Large in central jets, variable in } \\
\text { eccentric jets }\end{array}$ \\
\hline CW signal of regurgitant jet & Dense \\
\hline Other & $\begin{array}{l}\text { Holodiastolic flow reversal in } \\
\text { descending aorta }(E D V>20 \mathrm{~cm} / \mathrm{s})\end{array}$ \\
\hline \multicolumn{2}{|l|}{ Semiquantitative } \\
\hline Vena contracta width (mm) & $>6$ \\
\hline Pressure half-time $^{b}(\mathrm{~ms})$ & $<200$ \\
\hline \multicolumn{2}{|l|}{ Quantitative } \\
\hline $\mathrm{EROA}\left(\mathrm{mm}^{2}\right)$ & $\geq 30$ \\
\hline Regurgitant volume (mL/beat) & $\geq 60$ \\
\hline $\begin{array}{l}\text { Enlargement of cardiac } \\
\text { chambers }\end{array}$ & LV dilatation \\
\hline \multicolumn{2}{|c|}{$\begin{array}{l}\text { CW: continuous wave; EDV: end-diastolic velocity; EROA: effective } \\
\text { regurgitant orifice area; LV: left ventricle/left ventricular. a At a Nyquist } \\
\text { limit of } 50-60 \mathrm{~cm} / \mathrm{s} \text {. 'Pressure half-time is shortened with increasing LV } \\
\text { diastolic pressure, vasodilator therapy, and in patients with a dilated } \\
\text { compliant aorta, or lengthened in chronic aortic regurgitation. Adapted } \\
\text { from Lancellotti P et al. Recommendations for the echocardiographic } \\
\text { assessment of native valvular regurgitation: an executive summary from } \\
\text { the European Association of Cardiovascular Imaging. Eur Heart J } \\
\text { Cardiovasc Imaging 2013;14:611-644. Copyright (2013) by permission } \\
\text { of Oxford University Press on behalf of the European Society of } \\
\text { Cardiology. }\end{array}$} \\
\hline
\end{tabular}

result by TOE is mandatory in patients undergoing aortic valve preservation or repair.

\subsubsection{COMPUTED TOMOGRAPHY AND CARDIAC MAGNETIC RESONANCE}

CMR should be used to quantify the regurgitant fraction when echocardiographic measurements are equivocal or discordant with clinical findings. In patients with aortic dilatation, $\mathrm{CCT}$ is recommended to assess the maximum diameter at four levels, as in echocardiography. CMR can be used for follow-up, but indication for surgery should preferably be based on CCT measurements. Different methods of aortic measurements have been reported. To improve reproducibility, it is recommended to measure diameters using the inner-inner-edge technique at end diastole on the strictly transverse plane by double oblique reconstruction perpendicular to the axis of blood flow of the corresponding segment. Maximum root diameter should be taken from sinus-to-sinus diameter rather than sinus-to-commissure diameter, as it correlates more closely to longaxis leading-edge-to-leading-edge echo maximum diameters. ${ }^{103,104}$

\subsection{INDICATIONS FOR INTERVENTION}

Acute aortic regurgitation may require urgent surgery. It is mainly caused by infective endocarditis and aortic dissection but may also occur after blunt chest trauma and iatrogenic complications during catheter-based cardiac interventions. Specific guidelines deal with these entities. ${ }^{4,101}$ The recommendations on indications for surgery in severe aortic regurgitation and aortic root disease may be related to symptoms, status of the LV, or dilatation of the aorta [see table of recommendations on indications for surgery in severe aortic regurgitation and aortic root or tubular ascending aortic aneurysm (irrespective of the severity of aortic regurgitation), and Figure 2].

In symptomatic patients, surgery is recommended irrespective of the LVEF as long as aortic regurgitation is severe and the operative risk is not prohibitive. ${ }^{105-109}$ Surgery is recommended in symptomatic and asymptomatic patients with severe aortic regurgitation undergoing coronary artery bypass grafting (CABG), or surgery of the ascending aorta or another valve. ${ }^{110,111}$ In asymptomatic patients with severe aortic regurgitation, impairment of LV function [LVEF $\leq 50 \%$ or left ventricular end-systolic diameter (LVESD) $>50 \mathrm{~mm}]$ are associated with worse outcomes and surgery should therefore be pursued when these cut-offs are reached. ${ }^{107,108,112-114}$ LVESD should be related to body surface area (BSA) and a cut-off of $25 \mathrm{~mm} / \mathrm{m}^{2}$ BSA appeared to be more appropriate, especially in patients with small body size $\left(\mathrm{BSA}<1.68 \mathrm{~m}^{2}\right.$ ) or with large BSA who are not overweight. ${ }^{108,115}$ Some recent retrospective, nonrandomized studies emphasized the role of indexed LVESD and proposed a lower cut-off value of 20 or $22 \mathrm{~mm} / \mathrm{m}^{2}$ BSA for the indexed LVESD. ${ }^{116-118}$ One of these studies also suggests a higher cut-off value of $55 \%$ for LVEF. ${ }^{118}$ Based on these data, low-risk surgery may be discussed in some selected asymptomatic patients with LVESD $>20 \mathrm{~mm} / \mathrm{m}^{2}$ or resting LVEF between $50 \%$ and $55 \%$. In patients not reaching the thresholds for surgery, close follow-up is needed, and exercise testing should be liberally performed to identify borderline symptomatic patients. Progressive enlargement 


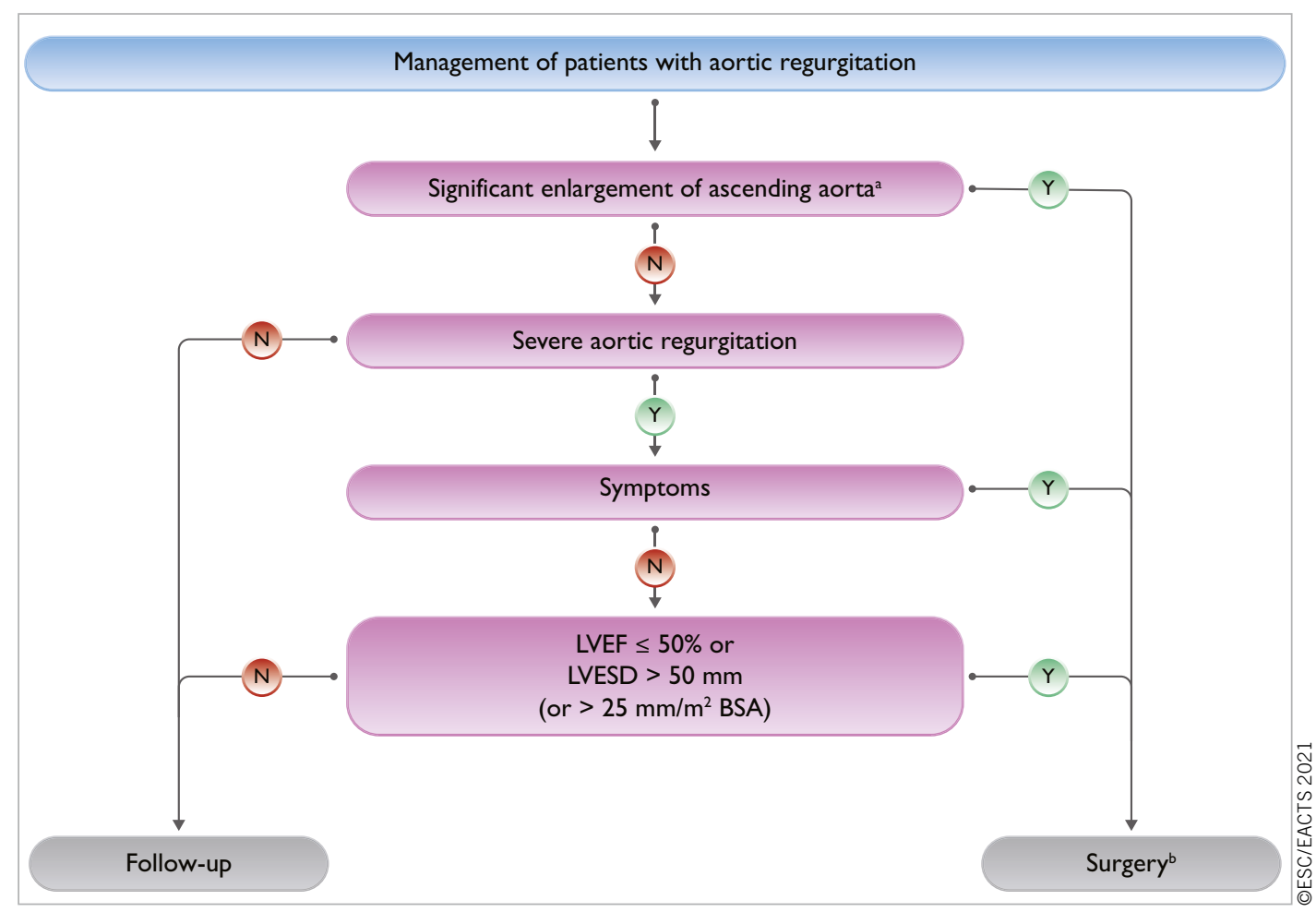

Figure 2. Management of patients with aortic regurgitation. BSA: body surface area; LV: left ventricle/left ventricular; LVESD: left ventricle end-systolic diameter; LVEF: left ventricular ejection fraction. ${ }^{a}$ See recommendations on indications for surgery in severe aortic regurgitation and aortic root disease for definition. ${ }^{b}$ Surgery should also be considered if significant changes in LV or aortic size occur during follow-up.

of the $\mathrm{LV}$, or a progressive decrease in its function in asymptomatic patients not reaching the thresholds for surgery but with significant LV dilatation [left ventricular end-diastolic diameter (LVEDD) $>65 \mathrm{~mm}$ ], may also be an appropriate indicator for timing operations in asymptomatic patients.

TAVI may be considered in experienced centres for selected patients with aortic regurgitation and ineligible for SAVR. ${ }^{119,120}$

In patients with a dilated aorta, the rationale for surgery has been best defined in patients with Marfan syndrome and root dilation. ${ }^{121,122}$ Root aneurysms require root replacement, with or without preservation of the native aortic valve. In contrast, tubular ascending aortic aneurysms in the presence of normal aortic valves require only a supracommissural tube graft replacement. In patients with aortic diameters borderline indicated for aortic surgery, the family history, age, and anticipated risk of the procedure should be taken into consideration. Irrespective of the degree of aortic regurgitation and type of valve pathology, in patients with an aortic diameter $\geq 55 \mathrm{~mm}$ with tricuspid or bicuspid aortic valves, ascending aortic surgery is recommended (see recommendations on indications for surgery in severe aortic regurgitation and aortic root disease) when the operative risk is not prohibitive. ${ }^{123-125}$ In individuals with bicuspid aortic valve, when additional risk factors or coarctation ${ }^{126}$ are present, surgery should be considered when aortic diameter is $\geq 50 \mathrm{~mm} .{ }^{127-129} \mathrm{In}$ all patients with Marfan syndrome, aortic surgery is recommended for a maximal aortic diameter $\geq 50 \mathrm{~mm} .^{5,121,122}$ When additional risk factors are present in patients with Marfan syndrome and in patients with a TGFBR1 or TGFBR2 mutation (including Loeys-Dietz syndrome), surgery should be considered at a maximal aortic diameter $\geq 45 \mathrm{~mm}^{121,130}$ and even earlier (aortic diameter of $40 \mathrm{~mm}$ or more) in women with low BSA, patients with a TGFBR2 mutation, or patients with severe extra-aortic features that appear to be at particularly high risk. ${ }^{130}$ For patients who have an indication for aortic valve surgery, an aortic diameter $\geq 45 \mathrm{~mm}$ is considered to indicate concomitant surgery of the aortic root or tubular ascending aorta. The patient's stature, the aetiology of the valvular disease (bicuspid valve), and the intraoperative shape and wall thickness of the ascending aorta should be considered for individual decisions.

The choice of the surgical procedure should be adapted according to the experience of the team, the presence of an aortic root aneurysm, characteristics of the cusps, life expectancy, and desired anticoagulation status.

Valve replacement is the standard procedure in the majority of patients with aortic regurgitation. Aortic valve-sparing root replacement and valve repair yield good long-term results in selected patients, with low rates of valve-related events as well as good quality of life $\mathrm{e}^{131-140}$ when performed in experienced centres. Aortic valve-sparing root replacement is recommended in younger patients who have an enlargement of the aortic root with normal cusp motion, when performed by experienced surgeons. ${ }^{133-136,140}$ In selected patients, aortic valve repair ${ }^{132,132,137}$ or the Ross procedure ${ }^{138,139}$ may be an alternative to valve replacement, when performed by experienced surgeons. 


\subsection{MEDICAL THERAPY}

Medical therapy, especially angiotensin-converting enzyme inhibitors (ACEI) or dihydropiridines, may provide symptomatic improvement in individuals with chronic severe aortic

\section{Recommendations on indications for surgery in $(A)$ severe aortic regurgitation and (B) aortic root or tubular ascending aortic aneurysm (irrespective of the severity of aortic regurgitation)}

Indications for surgery

A) Severe aortic regurgitation

Surgery is recommended in symptomatic patients regardless of LV function. $105-109$

Surgery is recommended in asymptomatic patients with LVESD $>50 \mathrm{~mm}$ or LVESD

$>25 \mathrm{~mm} / \mathrm{m}^{2}$ BSA (in patients with small body

size) or resting LVEF $\leq 50 \%$. ${ }^{107,108,112,114,115}$

Surgery may be considered in asymptomatic patients with LVESD $>20 \mathrm{~mm} / \mathrm{m}^{2}$ BSA (especially in patients with small body size) or resting LVEF $\leq 55 \%$, if surgery is at low risk.

Surgery is recommended in symptomatic and asymptomatic patients with severe aortic regurgitation undergoing $\mathrm{CABG}$ or surgery of the ascending aorta or of another valve.

Aortic valve repair may be considered in selected patients at experienced centres when durable results are expected.

B) Aortic root or tubular ascending aortic aneurys ${ }^{c}$ (irrespective of the severity of aortic regurgitation)

Valve-sparing aortic root replacement is

recommended in young patients with aortic root

dilation, if performed in experienced centres and durable results are expected. ${ }^{133-136,140}$

Ascending aortic surgery is recommended in patients with Marfan syndrome who have aortic root disease with a maximal ascending aortic diameter $\geq 50 \mathrm{~mm}$.

Ascending aortic surgery should be considered in patients who have aortic root disease with maximal ascending aortic diameter:

- $\geq 55 \mathrm{~mm}$ in all patients.

- $\geq 45 \mathrm{~mm}$ in the presence of Marfan syndrome and additional risk factors ${ }^{\mathrm{d}}$ or patients with a TGFBR1 or TGFBR2 mutation (including Loeys-Dietz syndrome). ${ }^{\mathrm{e}}$

- $\geq 50 \mathrm{~mm}$ in the presence of a bicuspid valve with additional risk factorsd or coarctation.

When surgery is primarily indicated for the aortic valve, replacement of the aortic root or tubular ascending aorta should be considered when $\geq 45 \mathrm{~mm}^{\mathrm{f}}$

I B

I

IIb

C

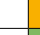

\begin{tabular}{|r} 
IIb \\
I
\end{tabular}

d

I

(n)

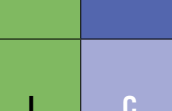



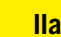

la

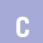

BSA: body surface area; CABG: coronary artery bypass grafting;

CCT: cardiac computed tomography; CMR: cardiac magnetic resonance; ECG: electrocardiogram; LV: left ventricle/left ventricular; LVEF: left ventricular ejection fraction; LVESD: left ventricular end-systolic diameter. ${ }^{a}$ Class of recommendation. ${ }^{\natural}$ Level of evidence. ${ }^{\text {CFor clinical }}$ decision making, dimensions of the aorta should be confirmed by ECG-gated CCT. 'Family history of aortic dissection (or personal history of spontaneous vascular dissection), severe aortic or mitral regurgitation, desire for pregnancy, uncontrolled systemic arterial hypertension and/or aortic size increase $>3 \mathrm{~mm} /$ year (using serial echocardiography or CMR measurements at the same level of the aorta confirmed by ECG-gated CCT). ${ }^{e} A$ lower threshold of $40 \mathrm{~mm}$ may be considered in women with Iow BSA, in patients with a TGFBR2 mutation or in patients with severe extra-aortic features. ${ }^{130}$ fConsidering age, BSA, aetiology of the valvular disease, presence of a bicuspid aortic valve, and intraoperative shape and thickness of the ascending aorta. regurgitation in whom surgery is not feasible. The value of ACEI or dihydropiridine in delaying surgery in the presence of moderate or severe aortic regurgitation in asymptomatic patients has not been established and their use is not recommended for this indication.

In patients who undergo surgery but continue to suffer from heart failure or hypertension, ACEI, angiotensin receptor blockers (ARBs), and beta-blockers are useful. ${ }^{141,142}$

In patients with Marfan syndrome, beta-blockers remain the mainstay for medical treatment and reducing shear stress and aortic growth rate and should be considered before and after surgery. ${ }^{143-145}$ While ARBs did not prove to have a superior effect when compared to beta-blockers, they may be considered as an alternative in patients intolerant to beta-blockers. ${ }^{146-148}$ By analogy, while there are no studies that provide supporting evidence, it is common clinical practice to advise beta-blocker or ARBs in patients with bicuspid aortic valve if the aortic root and/or ascending aorta is dilated. Management of aortic regurgitation during pregnancy is discussed in section 13.

\subsection{SERIAL TESTING}

All asymptomatic patients with severe aortic regurgitation and normal LV function should be followed up at least every year. In patients with either a first diagnosis or with LV diameter and/ or ejection fraction showing significant changes or approaching thresholds for surgery, follow-up should be continued at 3-6month intervals. Surgery may be considered in asymptomatic patients with significant LV dilatation (LVEDD $>65 \mathrm{~mm}$ ), and with progressive enlargement in the size of LV or progressive decrease of LVEF during follow-up. Patient's BNP levels could be of potential interest as a predictor of outcomes (particularly symptom onset and deterioration of LV function) and may be helpful in the follow-up of asymptomatic patients. ${ }^{149}$ Patients with mildto-moderate aortic regurgitation can be seen on a yearly basis and echocardiography performed every 2 years.

If the ascending aorta is dilated $(>40 \mathrm{~mm})$, it is recommended to systematically perform CCT or CMR. Follow-up assessment of the aortic dimension should be performed using echocardiography and/or CMR. Any increase $>3 \mathrm{~mm}$ should be validated by CCT angiography/CMR and compared with baseline data. After repair of the ascending aorta, Marfan patients remain at risk for dissection of the residual aorta and lifelong regular multidisciplinary follow-up at an expert centre is required.

\subsection{SPECIAL PATIENT POPULATIONS}

If aortic regurgitation requiring surgery is associated with severe primary and secondary mitral regurgitation, both should be treated during the same operation.

In patients with moderate aortic regurgitation who undergo CABG or mitral valve surgery, the decision to treat the aortic valve is controversial, as data show that progression of moderate aortic regurgitation is very slow in patients without aortic dilation. ${ }^{150}$ The Heart Team should decide based on the aetiology of 
aortic regurgitation, other clinical factors, the life expectancy of the patient, and the patient's operative risk.

The level of physical and sports activity in the presence of a dilated aorta remains a matter of clinical judgment in the absence of evidence. Current guidelines are very restrictive, particularly regarding isometric exercise, to avoid a catastrophic event. ${ }^{151}$ This approach is justified in the presence of connective tissue disease, but a more liberal approach is likely to be appropriate in other patients.

Given the familial risk of thoracic aortic aneurysms, screening and referral for genetic testing of the patient's first-degree relatives with appropriate imaging studies is indicated in patients with connective tissue disease. For patients with bicuspid valves, it is appropriate to have an echocardiographic screening of first-degree relatives.

\section{Aortic stenosis}

Aortic stenosis is the most common primary valve lesion requiring surgery or transcatheter intervention in Europe ${ }^{1}$ and North America. Its prevalence is rising rapidly as a consequence of the ageing population. ${ }^{2,152}$

\subsection{EVALUATION}

\subsubsection{ECHOCARDIOGRAPHY}

Echocardiography is key to confirming the diagnosis and severity of aortic stenosis, assessing valve calcification, LV function and wall thickness, detecting other valve disease or aortic pathology, and providing prognostic information. ${ }^{43,153,154}$ Assessment should be undertaken when blood pressure (BP) is well controlled to avoid the confounding flow effects of increased afterload. New echocardiographic parameters, stress imaging and CCT provide important adjunctive information when severity is uncertain (Figure 3 ).

Current international recommendations for the echocardiographic evaluation of patients with aortic stenosis ${ }^{25}$ depend upon measurement of mean pressure gradient (the most robust parameter), peak transvalvular velocity $\left(\mathrm{V}_{\max }\right)$, and valve area. Although valve area is the theoretically ideal measurement for assessing severity, there are numerous technical limitations. Clinical decision making in discordant cases should therefore take account of additional parameters: functional status, stroke volume, Doppler velocity index, ${ }^{156}$ degree of valve calcification, LV function, the presence or absence of LV hypertrophy, flow conditions, and the adequacy of BP control. ${ }^{25}$ Low flow is arbitrarily defined by a stroke volume index (SVi) $\leq 35 \mathrm{~mL} / \mathrm{m}^{2}-$ a threshold that is under current debate. ${ }^{155,157,158}$ The use of sex -specific thresholds has been recently proposed. ${ }^{159}$ Four broad categories can be defined:

- High-gradient aortic stenosis [mean gradient $\geq 40 \mathrm{mmHg}$, peak velocity $\geq 4.0 \mathrm{~m} / \mathrm{s}$, valve area $\leq 1 \mathrm{~cm}^{2}$ (or $\left.\left.\leq 0.6 \mathrm{~cm}^{2} / \mathrm{m}^{2}\right)\right]$. Severe aortic stenosis can be assumed irrespective of LV function and flow conditions.

- Low-flow, low-gradient aortic stenosis with reduced ejection fraction (mean gradient $<40 \mathrm{mmHg}$, valve area $\leq 1 \mathrm{~cm}^{2}$, LVEF $\left.<50 \%, \mathrm{SVi} \leq 35 \mathrm{~mL} / \mathrm{m}^{2}\right)$. Low-dose dobutamine stress echocardiography (DSE) is recommended to distinguish between true severe and pseudo-severe aortic stenosis (increase in valve area to $>1.0 \mathrm{~cm}^{2}$ with increased flow) and identify patients with no flow (or contractile) reserve. ${ }^{160}$ However, utility in elderly patients has only been evaluated in small registries. ${ }^{161}$

- Low-flow, low-gradient aortic stenosis with preserved ejection fraction (mean gradient $<40 \mathrm{mmHg}$, valve area $\leq 1 \mathrm{~cm}^{2}$, $\mathrm{LVEF} \geq 50 \%, \mathrm{SVi} \leq 35 \mathrm{~mL} / \mathrm{m}^{2}$ ). Typically encountered in hypertensive elderly subjects with small LV size and marked hypertrophy. ${ }^{157,162}$ This scenario may also result from conditions associated with low stroke volume (e.g. moderate/severe mitral regurgitation, severe tricuspid regurgitation, severe mitral stenosis, and large ventricular septal defect and severe RV dysfunction). Diagnosis of severe aortic stenosis is challenging and requires careful exclusion of measurement errors and other explanations for the echocardiographic findings, ${ }^{25}$ as well as the presence or absence of typical symptoms (with no other explanation), LV hypertrophy (in the absence of coexistent hypertension) or reduced LV longitudinal strain (with no other cause). CCT assessment of the degree of valve calcification provides important additional information [thresholds (Agatston units) for severe aortic stenosis: men $>3000$, women $>1600=$ highly likely; men $>2000$, women $>1200=$ likely; men $<1600$, women $<800=$ unlikely]. . $^{35,36,163,164}$

- Normal-flow, low-gradient aortic stenosis with preserved ejection fraction (mean gradient $<40 \mathrm{mmHg}$, valve area $\leq 1 \mathrm{~cm}^{2}$, $\mathrm{LVEF} \geq 50 \%, \mathrm{SVi}>35 \mathrm{~mL} / \mathrm{m}^{2}$ ). These patients usually have only moderate aortic stenosis. ${ }^{36,165-167}$

\subsubsection{ADDITIONAL DIAGNOSTIC AND PROGNOSTIC PARAMETERS}

The resting Doppler velocity index (DVI, also termed 'dimensionless index') - the ratio of the left ventricular outflow tract (LVOT) time-velocity integral (TVI) to that of the aortic valve jet - does not require calculation of LVOT area and may assist evaluation when other parameters are equivocal (a value $<0.25$ suggests that severe aortic stenosis is highly likely). ${ }^{156}$ Assessment of global longitudinal strain provides additional information concerning LV function and a threshold of $15 \%$ may help to identify patients with severe asymptomatic aortic stenosis who are at higher risk of clinical deterioration or premature mortality. ${ }^{26,168}$ TOE allows evaluation of concomitant mitral valve disease and may be of value for periprocedural imaging during TAVI and SAVR. ${ }^{169}$

Natriuretic peptides predict symptom-free survival and outcome in normal and low-flow severe aortic stenosis. ${ }^{170,171}$ They can be used to arbitrate the source of symptoms in patients with multiple potential causes and identify those with high-risk asymptomatic aortic stenosis who may benefit from early intervention (section 5.2.2, Table 6 and Figure 3).

Exercise testing may unmask symptoms and is recommended for risk stratification of asymptomatic patients with severe aortic stenosis. ${ }^{172}$ Exercise echocardiography provides additional prognostic information by assessing the increase in mean pressure gradient and change in LV function. ${ }^{173}$ 


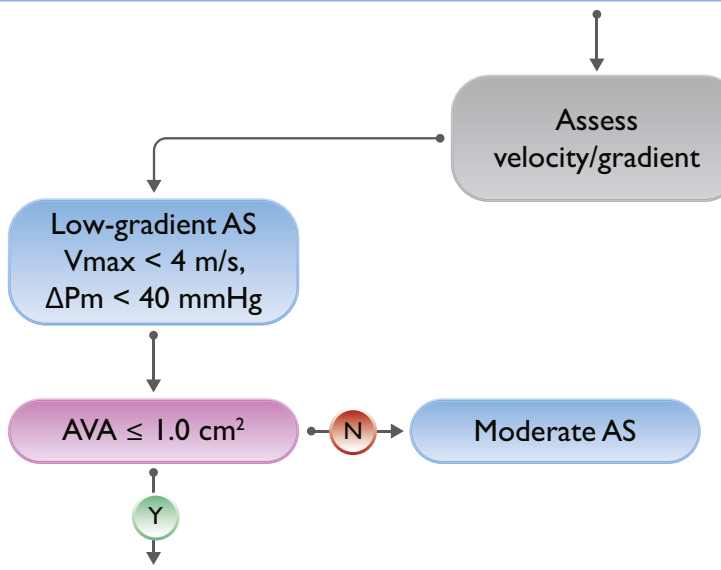

Check blood pressure and exclude measurement errors that may cause underestimation of gradient, flow or AVA
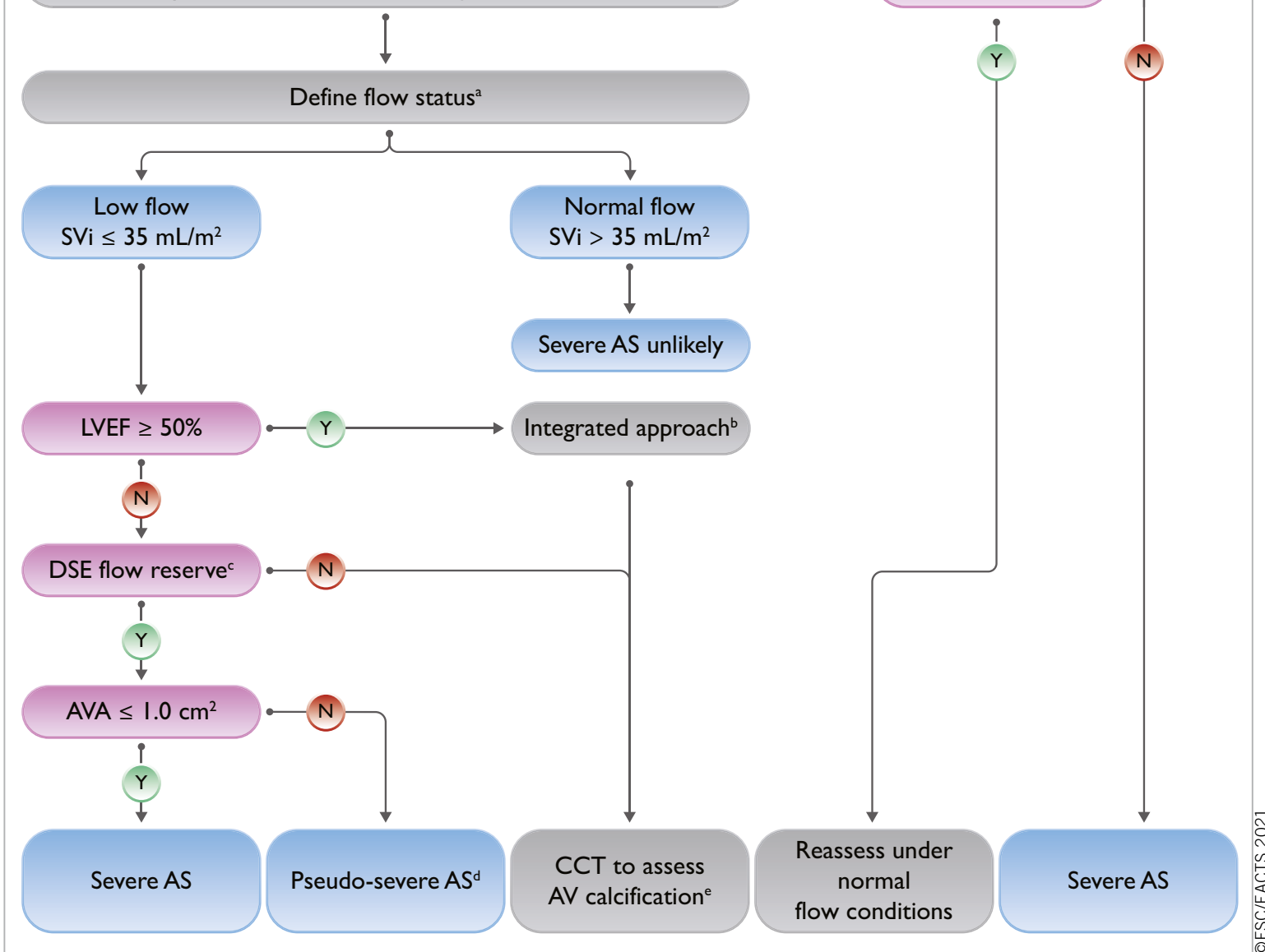

Figure 3. Integrated imaging assessment of aortic stenosis. AS: aortic stenosis; AV: aortic valve; AVA: aortic valve area; CT: computed tomography; $\triangle P$ m: mean pressure gradient; DSE: dobutamine stress echocardiography; LV: left ventricle/left ventricular; LVEF: left ventricular ejection fraction; SVi: stroke volume index; $V_{\text {max }}$ : peak transvalvular velocity. ${ }^{a}$ High flow may be reversible in patients with anaemia, hyperthyroidism or arterio-venous fistulae, and may also be present in patients with hypertrophic obstructive cardiomyopathy. Upper limit of normal flow using pulsed Doppler echocardiography: cardiac index $4.1 \mathrm{~L} / \mathrm{min} / \mathrm{m}^{2}$ in men and women, SVi $54 \mathrm{~mL} / \mathrm{m}^{2}$ in $\mathrm{men}$, $51 \mathrm{~mL} / \mathrm{m}^{2}$ in women). ${ }^{155}$ b Consider also: typical symptoms (with no other explanation), LV hypertrophy (in the absence of coexistent hypertension) or reduced LV longitudinal function (with no other cause). ${ }^{c} D S E$ flow reserve: $>20 \%$ increase in stroke volume in response to low-dose dobutamine. ${ }^{d}$ Pseudo-severe aortic stenosis: AVA $>1.0 \mathrm{~cm}^{2}$ with increased flow. ${ }^{e}$ Thresholds for severe aortic stenosis assessed by means of CT measurement of aortic valve calcification (Agatston units): men >3000, women >1600: highly likely; men >2000, women >1200: likely; men <1600, women <800: unlikely. 
Table 6. Clinical, anatomical and procedural factors that influence the choice of treatment modality for an individual patient.

\begin{tabular}{|c|c|c|}
\hline & $\begin{array}{c}\text { Favours } \\
\text { TAVI }\end{array}$ & $\begin{array}{c}\text { Favours } \\
\text { SAVR }\end{array}$ \\
\hline \multicolumn{3}{|l|}{ Clinical characteristics } \\
\hline Lower surgical risk & - & + \\
\hline Higher surgical risk & + & - \\
\hline Younger age $\mathrm{e}^{\mathrm{a}}$ & - & + \\
\hline Older age $^{\mathrm{a}}$ & + & - \\
\hline $\begin{array}{l}\text { Previous cardiac surgery (particularly intact } \\
\text { coronary artery bypass grafts at risk of } \\
\text { injury during repeat sternotomy) }\end{array}$ & + & - \\
\hline Severe frailty ${ }^{b}$ & + & - \\
\hline Active or suspected endocarditis & - & + \\
\hline
\end{tabular}

Anatomical and procedural factors

\begin{tabular}{|l|c|c|}
\hline TAVI feasible via transfemoral approach & + & - \\
\hline $\begin{array}{l}\text { Transfemoral access challenging or } \\
\text { impossible and SAVR feasible }\end{array}$ & - & + \\
\hline
\end{tabular}

Transfemoral access challenging or impossible and SAVR inadvisable

Sequelae of chest radiation

Porcelain aorta

High likelihood of severe patient-prosthesis mismatch (AVA $<0.65 \mathrm{~cm}^{2} / \mathrm{m}^{2} \mathrm{BSA}$ )

\begin{tabular}{|l|c|c|}
\hline Severe chest deformation or scoliosis & + & - \\
\hline $\begin{array}{l}\text { Aortic annular dimensions unsuitable for } \\
\text { available TAVI devices }\end{array}$ & - & + \\
\hline Bicuspid aortic valve & - & + \\
\hline $\begin{array}{l}\text { Valve morphology unfavourable for TAVI } \\
\text { (e.g. high risk of coronary obstruction due } \\
\text { to low coronary ostia or heavy leaflet/LVOT } \\
\text { calcification) }\end{array}$ & - & + \\
\hline Thrombus in aorta or LV & - & + \\
\hline
\end{tabular}

Concomitant cardiac conditions requiring intervention

Significant multi-vessel CAD requiring surgical revascularization ${ }^{d}$

Severe primary mitral valve disease

Severe tricuspid valve disease

Significant dilatation/aneurysm of the aortic root and/or ascending aorta

Septal hypertrophy requiring myectomy

AVA: aortic valve area, BSA: body surface area, CAD: coronary artery disease; ESC: European Society of Cardiology; LV: left ventricle/left ventricular; LVOT: left ventricular outflow tract; SAVR: surgical aortic valve replacement; TAVI: transcatheter aortic valve implantation.

Integration of these factors provides guidance for the Heart Team decision (indications for intervention are provided in the table of recommendations on indications for intervention in symptomatic and asymptomatic aortic stenosis and recommended mode of intervention). a Life expectancy is highly dependent on absolute age and frailty, differs between men and women, and may be a better guide than age alone.

There is wide variation across Europe and elsewhere in the world (http:// ghdx.healthdata.org/record/ihme-data/gbd-2017-life-tables-1950-2017). bSevere frailty: $>2$ factors according to Katz index ${ }^{59}$ (see section 3.3 for further discussion). 'Via non-transfemoral approach. ${ }^{d}$ According to the 2019 ESC Guidelines for the diagnosis and management of chronic coronary syndromes.
CCT provides information concerning the anatomy of the aortic root and ascending aorta, and the extent and distribution of valve and vascular calcification, and feasibility of vascular access. ${ }^{174}$ Quantification of valve calcification predicts disease progression and clinical events ${ }^{164}$ and may be useful when combined with geometric assessment of valve area in assessing the severity of aortic stenosis in patients with low valve gradient. ${ }^{35,36,163,164}$

Myocardial fibrosis is a major driver of LV decompensation in aortic stenosis (regardless of the presence or absence of CAD), which can be detected and quantified using CMR. Amyloidosis is also frequently associated with aortic stenosis in elderly patients (incidence 9-15\%). ${ }^{175}$ When cardiac amyloidosis is clinically suspected, based on symptoms (neuropathy and hematologic data), diphosphonate scintigraphy and/or CMR should be considered. Both entities persist following valve intervention and are associated with poor long-term prognosis. ${ }^{176-179}$

Coronary angiography is essential prior to TAVI and SAVR to determine the potential need for concomitant revascularization (see section 3.2.4.1 and section 5.5). Retrograde LV catheterization is not recommended unless there are symptoms and signs of severe aortic stenosis and non-invasive investigations are inconclusive.

\subsubsection{TAVI DIAGNOSTIC WORKUP}

Prior to TAVI, CCT is the preferred imaging tool to assess: (i) aortic valve anatomy, (ii) annular size and shape, (iii) extent and distribution of valve and vascular calcification, (iv) risk of coronary ostial obstruction, (v) aortic root dimensions, (vi) optimal fluoroscopic projections for valve deployment, and (vii) feasibility of vascular access (femoral, subclavian, axillary, carotid, transcaval or transapical). Adverse anatomical findings may suggest that SAVR is a better treatment option (Table 6). TOE is more operator-dependent but may be considered when CCT is difficult to interpret or relatively contraindicated (e.g. chronic renal failure).

\subsection{INDICATIONS FOR INTERVENTION (SAVR OR TAVI)}

Indications for aortic valve intervention are summarized in the table of recommendations on indications for intervention in symptomatic and asymptomatic aortic stenosis and recommended mode of intervention and in Figure 4.

\subsubsection{SYMPTOMATIC AORTIC STENOSIS}

Symptomatic severe aortic stenosis has dismal prognosis and early intervention is strongly recommended in all patients. The only exceptions are for those in whom intervention is unlikely to improve quality of life or survival (due to severe comorbidities) or for those with concomitant conditions associated with survival $<1$ year (e.g. malignancy) (section 3).

Intervention is recommended in symptomatic patients with high-gradient aortic stenosis, regardless of LVEF. However, management of patients with low-gradient aortic stenosis is more challenging:

- LV function usually improves after intervention in patients with low-flow, low-gradient aortic stenosis, when reduced ejection fraction is predominantly caused by excessive afterload. ${ }^{32,180}$ Conversely, improvement is uncertain if the primary cause of 


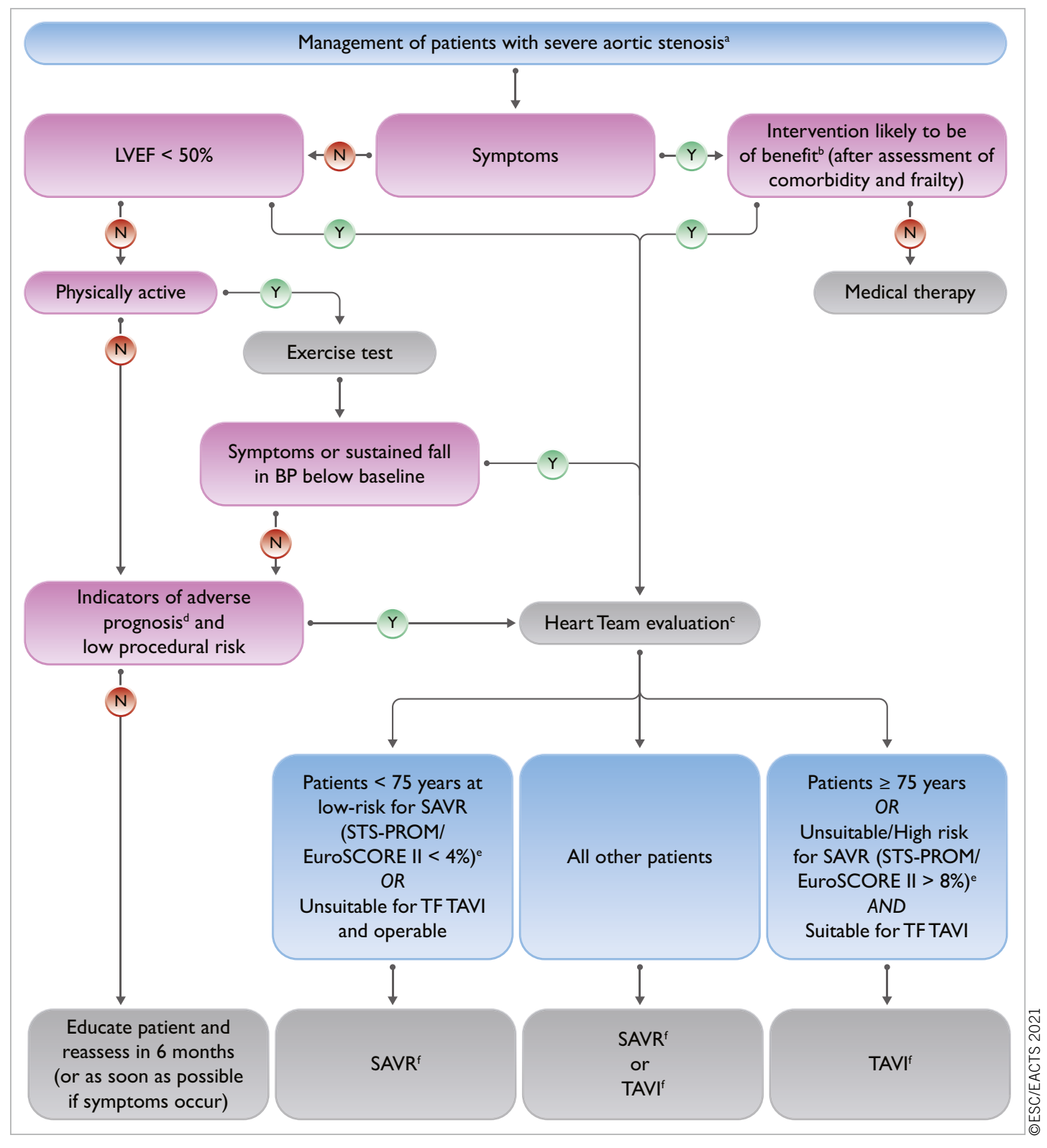

Figure 4. Management of patients with severe aortic stenosis. BP: blood pressure; EuroSCORE: European System for Cardiac Operative Risk Evaluation; LVEF: left ventricular ejection fraction; SAVR: surgical aortic valve replacement; STS-PROM: Society of Thoracic Surgeons - predicted risk of mortality; TAVI: transcatheter aortic valve implantation; TF: transfemoral. ${ }^{a}$ See Figure 3: Integrated imaging assessment of aortic stenosis. ${ }^{b}$ Prohibitive risk is defined in Supplementary Table 5. ${ }^{c}$ Heart Team assessment based upon careful evaluation of clinical, anatomical, and procedural factors (see Table 6 and table on Recommendations on indications for intervention in symptomatic and asymptomatic aortic stenosis and recommended mode of intervention). The Heart Team recommendation should be discussed with the patient who can then make an informed treatment choice. ${ }^{d}$ Adverse features according to clinical, imaging (echocardiography/CT), and/or biomarker assessment. ${ }^{e}$ STS-PROM: http://riskcalc.sts.org/stswebriskcalc/\#/calculate, EuroSCORE II: http://www.euroscore.org/calc.html. ${ }^{f}$ If suitable for procedure according to clinical, anatomical, and procedural factors (Table 6).

reduced ejection fraction is scarring due to myocardial infarction or cardiomyopathy. Intervention is recommended when severe aortic stenosis is confirmed by stress echocardiography (true severe aortic stenosis; Figure 3), ${ }^{32}$ while patients with pseudo-severe aortic stenosis should receive conventional heart failure treatment. ${ }^{142,181}$ The presence or absence of flow reserve (increase in stroke volume $\geq 20 \%$ ) $\mathrm{d}^{\mathrm{oe}} \mathrm{s}$ not appear to influence prognosis in contemporary series of patients undergoing TAVI or SAVR, ${ }^{182-184}$ and although those with no flow reserve show increased procedural mortality, both modes of intervention improve ejection fraction and clinical outcomes..$^{32,180,182}$ Decision making for such patients should take account of comorbidities, degree of valve calcification, extent of CAD, and feasibility of revascularization. 
- Data concerning the natural history of low-flow, low-gradient aortic stenosis and preserved ejection fraction, and outcomes after SAVR and TAVI remain controversial. ${ }^{162,165,167}$ Intervention should only be considered in those with symptoms and significant valve obstruction (see table of recommendations on indications for intervention in symptomatic and asymptomatic aortic stenosis and recommended mode of intervention and Figure 4).

- The prognosis of patients with normal-flow, low-gradient aortic stenosis and preserved ejection fraction is similar to that of moderate aortic stenosis - regular clinical and echocardiographic surveillance is recommended. ${ }^{165,166,185}$

\subsubsection{ASYMPTOMATIC AORTIC STENOSIS}

Intervention is recommended in asymptomatic patients with severe aortic stenosis and impaired LV function of no other cause, ${ }^{9}$ and those who are asymptomatic during normal activities but develop symptoms during exercise testing. ${ }^{172,186}$ Management of asymptomatic severe aortic stenosis is otherwise controversial and the decision to intervene requires careful assessment of the benefits and risks in an individual patient.

In the absence of adverse prognostic features, watchful waiting has generally been recommended with prompt intervention at symptom onset. ${ }^{187}$ Data from a single RCT have shown significant reduction in the primary endpoint (death during or within 30 days of surgery or cardiovascular death during the entire follow-up period) following early SAVR compared with conservative management $[1 \%$ vs. $15 \%$; hazard ratio 0.09 ; $95 \%$ confidence interval (CI), 0.01-0.67; $\mathrm{P}=0.003] .{ }^{188}$ However, subjects were selected per inclusion criteria (median age 64 years, minimal comorbidities, low operative risk) and follow-up in the conservative group was limited. Further randomized trials [EARLY TAVR (NCT03042104), AVATAR (NCT02436655), EASY-AS (NCT04204915), EVOLVED (NCT03094143)] will help determine future recommendations.

Predictors of symptom development and adverse outcomes in asymptomatic patients include clinical characteristics (older age, atherosclerotic risk factors), echocardiographic parameters (valve calcification, peak jet velocity ${ }^{189,190}$ ), LVEF, rate of haemodynamic progression, ${ }^{189}$ increase in mean gradient $>20 \mathrm{mmHg}$ with exercise, ${ }^{172}$ severe LV hypertrophy, ${ }^{191}$ indexed stroke volume, ${ }^{158}$ LA volume, ${ }^{192}$ LV global longitudinal strain, ${ }^{26,168,193}$ and abnormal biomarker levels (natriuretic peptides, troponin, and fetuin-A). ${ }^{170,171,194,195}$ Early intervention may be considered in asymptomatic patients with severe aortic stenosis and one or more of these predictors if procedural risk is low (although application of TAVI in this setting has yet to be formally evaluated) (Table 6 and Figure 4). Otherwise, watchful waiting is a safer and more appropriate strategy.

\subsubsection{THE MODE OF INTERVENTION}

Use of SAVR and TAVI as complementary treatment options has allowed a substantial increase in the overall number of patients with aortic stenosis undergoing surgical or transcatheter intervention in the past decade. ${ }^{196}$ RCTs have assessed the two modes of intervention across the spectrum of surgical risk in predominantly elderly patients and a detailed appraisal of the evidence base is provided in Supplementary Section 5. In brief, these trials used surgical risk scores to govern patient selection and demonstrate that TAVI is superior to medical therapy in extreme-risk patients, ${ }^{197}$ and noninferior to SAVR in high- ${ }^{198-201}$ and intermediate-risk patients at follow-up extending to 5 years. ${ }^{202-208}$ The more recent PARTNER 3 and Evolut Low Risk trials demonstrate that TAVI is non-inferior to SAVR in low-risk patients at 2-year follow-up. ${ }^{209-212}$ Importantly, patients in the low-risk trials were predominantly male and relatively elderly (e.g. PARTNER 3: mean age 73.4 years, $<70$ years $24 \%, 70-75$ years $36 \%,>75$ years $40 \%$, >80 years $13 \%$ ) whilst those with low-flow aortic stenosis or adverse anatomical characteristics for either procedure (including bicuspid aortic valves or complex coronary disease) were excluded.

Rates of vascular complications, pacemaker implantation, and paravalvular regurgitation are consistently higher after TAVI, whereas severe bleeding, acute kidney injury, and new-onset AF are more frequent after SAVR. Although the likelihood of paravalvular regurgitation has been reduced with newer transcatheter heart valve designs, pacemaker implantation (and new-onset left bundle branch block) may have long-term consequences ${ }^{213-215}$ and further refinements are required. Most patients undergoing TAVI have a swift recovery, short hospital stay, and rapidly return to normal activities. ${ }^{216,217}$ Despite these benefits, there is wide variation in worldwide access to the procedure as a result of high device costs and differing levels of healthcare resources. ${ }^{71,218,219}$

The Task Force has attempted to address the gaps in evidence and provide recommendations concerning the indications for intervention and mode of treatment (Recommendations on indications for intervention in symptomatic and asymptomatic aortic stenosis and recommended mode of intervention, Figure 4) that are guided by the RCT findings and compatible with real-world Heart Team decision making for individual patients (many of whom fall outside the RCT inclusion criteria). Aortic stenosis is a heterogeneous condition and selection of the most appropriate mode of intervention should be carefully considered by the Heart Team for all patients, accounting for individual age and estimated life expectancy, comorbidities (including frailty and overall quality of life, section 3), anatomical and procedural characteristics (Table 6), the relative risks of SAVR and TAVI and their long-term outcomes, prosthetic heart valve durability, feasibility of transfemoral TAVI, and local experience and outcome data. These factors should be discussed with the patient and their family to allow informed treatment choice.

The interplay between estimated life expectancy and prosthetic heart valve durability is a key consideration in these discussions. Age is a surrogate for life expectancy but had no impact on the outcomes of the low-risk RCTs at 1-2 year follow-up. Life expectancy varies widely across the world and is highly dependent on absolute age, sex, frailty, and the presence of comorbidities (http://ghdx.healthdata.org/record/ihme-data/gbd2017-life-tables-1950-2017); it may be a better guide than age alone but is difficult to determine in individual patients. Although 
some (now abandoned) surgical bioprosthetic designs have failed early, the durability of contemporary surgical bioprosthetic valves beyond 10 years is well established. ${ }^{220}$ Conversely, registry data provide some reassurance concerning the long-term durability of TAVI devices up to 8 years but largely relate to older high-/intermediate-risk patients, ${ }^{221-224}$ whereas information concerning durability in low-risk patients is currently limited to 2-year follow-up.

\section{Recommendations on indications for intervention ${ }^{\mathrm{a}}$ in symptomatic (A) and asymptomatic (B) aortic stenosis and recommended mode of intervention (C)}

\begin{tabular}{|c|c|c|}
\hline A) Symptomatic aortic stenosis & Class $^{b}$ & Level ${ }^{\mathrm{c}}$ \\
\hline $\begin{array}{l}\text { Intervention is recommended in symptomatic } \\
\text { patients with severe, high-gradient aortic stenosis } \\
\text { [mean gradient } \geq 40 \mathrm{mmHg} \text {, peak velocity } \\
\geq 4.0 \mathrm{~m} / \mathrm{s} \text {, and valve area } \leq 1.0 \mathrm{~cm}^{2} \text { (or } \\
\leq 0.6 \mathrm{~cm}^{2} / \mathrm{m}^{2} \text { )]. } .^{235,236}\end{array}$ & $\mathbf{I}$ & B \\
\hline $\begin{array}{l}\text { Intervention is recommended in symptomatic } \\
\text { patients with severe low-flow }\left(\mathrm{SVi} \leq 35 \mathrm{~mL} / \mathrm{m}^{2}\right) \\
\text { low-gradient }(<40 \mathrm{mmHg} \text { ) aortic stenosis with } \\
\text { reduced ejection fraction }(<50 \%) \text {, and evidence } \\
\text { of flow (contractile) reserve. }{ }^{32,237}\end{array}$ & I & B \\
\hline $\begin{array}{l}\text { Intervention should be considered in } \\
\text { symptomatic patients with low-flow, low-gradient } \\
(<40 \mathrm{mmHg} \text { ) aortic stenosis with normal ejection } \\
\text { fraction after careful confirmation that the aortic } \\
\text { stenosis is severed (Figure } 3) \text {. }\end{array}$ & Ila & C \\
\hline $\begin{array}{l}\text { Intervention should be considered in } \\
\text { symptomatic patients with low-flow, low-gradient } \\
\text { severe aortic stenosis and reduced ejection } \\
\text { fraction without flow (contractile) reserve, } \\
\text { particularly when CCT calcium scoring confirms } \\
\text { severe aortic stenosis. }\end{array}$ & Ila & C \\
\hline $\begin{array}{l}\text { Intervention is not recommended in patients with } \\
\text { severe comorbidities when the intervention is } \\
\text { unlikely to improve quality of life or prolong } \\
\text { survival }>1 \text { year. }\end{array}$ & III & C \\
\hline \multicolumn{3}{|l|}{ B) Asymptomatic patients with severe aortic stenosis } \\
\hline $\begin{array}{l}\text { Intervention is recommended in asymptomatic } \\
\text { patients with severe aortic stenosis and systolic } \\
\text { LV dysfunction (LVEF }<50 \% \text { ) without another } \\
\text { cause. } ., 238,239\end{array}$ & I & B \\
\hline $\begin{array}{l}\text { Intervention is recommended in asymptomatic } \\
\text { patients with severe aortic stenosis and } \\
\text { demonstrable symptoms on exercise testing. }\end{array}$ & I & C \\
\hline $\begin{array}{l}\text { Intervention should be considered in } \\
\text { asymptomatic patients with severe aortic stenosis } \\
\text { and systolic LV dysfunction (LVEF <55\%) without } \\
\text { another cause. } \text {, }^{9,240,241}\end{array}$ & Ila & B \\
\hline $\begin{array}{l}\text { Intervention should be considered in } \\
\text { asymptomatic patients with severe aortic stenosis } \\
\text { and a sustained fall in BP ( }>20 \mathrm{mmHg} \text { ) during } \\
\text { exercise testing. }\end{array}$ & Ila & C \\
\hline $\begin{array}{l}\text { Intervention should be considered in } \\
\text { asymptomatic patients with LVEF }>55 \% \text { and } \\
\text { a normal exercise test if the procedural risk is low } \\
\text { and one of the following parameters is present: } \\
\text { - Very severe aortic stenosis (mean gradient } \\
\geq 60 \mathrm{mmHg} \text { or } \mathrm{V}_{\text {max }}>5 \mathrm{~m} / \mathrm{s} \text { )., }{ }^{9,242} \\
\text { - Severe valve calcification (ideally assessed by } \\
\mathrm{CCT} \text { ) and } \mathrm{V}_{\text {max }} \text { progression } \geq 0.3 \mathrm{~m} / \mathrm{s} / \\
\text { year. }{ }^{164,189,243} \\
\text { - Markedly elevated BNP levels ( }>3 \text { - age- and } \\
\text { sex-corrected normal range) confirmed by } \\
\text { repeated measurements and without other } \\
\text { explanation. }{ }^{163,171}\end{array}$ & Ila & B \\
\hline
\end{tabular}

\section{C) Mode of intervention}

Aortic valve interventions must be performed in Heart Valve Centres that declare their local expertise and outcomes data, have active interventional cardiology and cardiac surgical programmes on site, and a structured collaborative Heart Team approach.

The choice between surgical and transcatheter intervention must be based upon careful evaluation of clinical, anatomical, and procedural factors by the Heart Team, weighing the risks and benefits of each approach for an individual patient. The Heart Team recommendation should be discussed with the patient who can then make an informed treatment choice.

SAVR is recommended in younger patients who are low risk for surgery $\left(<75\right.$ years $^{e}$ and STSPROM/ EuroSCORE II $<4 \%)^{\mathrm{e}, \mathrm{f}}$, or in patients who are operable and unsuitable for transfemoral TAVI. ${ }^{244}$

TAVI is recommended in older patients ( $\geq 75$ years), or in those who are high risk (STSPROM/ EuroSCORE II $>8 \%$ ) or unsuitable for surgery. ${ }^{197-206,245}$

SAVR or TAVI are recommended for remaining patients according to individual clinical, anatomical, and procedural

characteristics. ${ }^{202-205,207,209,210,212 \mathrm{f,g}}$

Non-transfemoral TAVI may be considered in patients who are inoperable and unsuitable for transfemoral TAVI.

Balloon aortic valvotomy may be considered as a bridge to SAVR or TAVI in haemodynamically unstable patients and (if feasible) in those with severe aortic stenosis who require urgent highrisk NCS (Figure 11).

D) Concomitant aortic valve surgery at the time of other cardiac/ascending aorta surgery

SAVR is recommended in patients with severe aortic stenosis undergoing $C A B G$ or surgical intervention on the ascending aorta or another valve.

SAVR should be considered in patients with moderate aortic stenosish undergoing CABG or surgical intervention on the ascending aorta or another valve after Heart Team discussion.

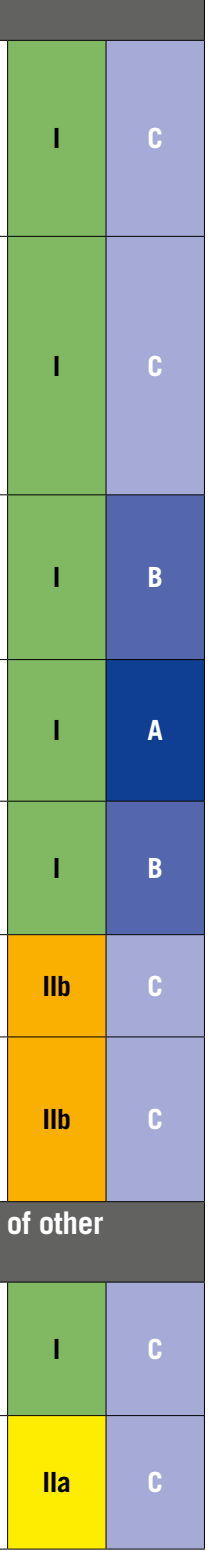

BNP: B-type natriuretic peptide; BP: blood pressure; CABG: coronary artery bypass grafting; CCT: cardiac computed tomography; EuroSCORE: European System for Cardiac Operative Risk Evaluation; LV: left ventricle/left ventricular; LVEF: left ventricular ejection fraction; NCS: non-cardiac surgery; SAVR: surgical aortic valve replacement; STS-PROM: Society of Thoracic Surgeons - predicted risk of mortality; SVi: stroke volume index; TAVI: transcatheter aortic valve implantation; $\mathrm{V}_{\text {max }}$ : peak transvalvular velocity. aSAVR or TAVI. ${ }^{\mathrm{b}}$ Class of recommendation. ${ }^{\mathrm{m}}$ Level of evidence. ${ }^{\mathrm{d}}$ Explanations other than severe aortic stenosis for a small valve area but low gradient despite preserved LVEF are frequent and must be carefully excluded (Figure $\mathbf{3}$ ).

eSTS-PROM: http://riskcalc.sts.org/stswebriskcalc/\#/calculate, EuroSCORE II: http://www.euroscore.org/calc.html. flf suitable for surgery (see Table 6). ${ }^{\mathrm{g}}$ If suitable for transfemoral TAVI (see Table 6).

${ }^{\mathrm{h}}$ Moderate aortic stenosis is defined as a valve area of $1.0-1.5 \mathrm{~cm}^{2}$ (or mean aortic gradient of $25-40 \mathrm{mmHg}$ ) in normal flow conditions - clinical assessment is essential to determine whether SAVR is appropriate for an individual patient.

Data comparing the durability of transcatheter heart valves and surgical bioprostheses directly remain limited. Rates of aortic valve re-intervention were higher after TAVI using a balloonexpandable valve compared to SAVR at 5-year follow-up in the PARTNER 2A trial (3.2\% vs. 0.8\%; hazard ratio, 3.3; 95\% CI, 
1.3-8.1), ${ }^{206}$ whereas rates of structural valve deterioration (SVD) were not statistically different following SAVR and TAVI using the third generation SAPIEN 3 device in a parallel observational registry over the same time frame..$^{225}$

Valve-in-valve TAVI is an established treatment option for surgical bioprosthetic valve deterioration but may not be appropriate or feasible in all patients due to the increased likelihood of PPM in patients with a small aortic root (or undersized original prosthesis), incompatible surgical valve designs associated with increased risk of coronary occlusion, or difficult vascular access; re-do SAVR should also be considered in these settings. ${ }^{226-228}$ Favourable short-term outcomes of redo-TAVI have been demonstrated in selected older patients with transcatheter heart valve deterioration, ${ }^{229}$ despite theoretical concerns relating to maintained coronary access. ${ }^{230}$

A bicuspid aortic valve is more frequent in younger patients with aortic stenosis. While several registries have reported excellent outcomes of TAVI in patients with a bicuspid valve who were unsuitable for surgery, ${ }^{231-233}$ SAVR remains more appropriate in patients with aortic stenosis affecting a bicuspid valve and in those with associated disease (e.g. aortic root dilatation, complex coronary disease, or severe mitral regurgitation) requiring a surgical approach.

In summary, prosthetic heart valve durability is a key consideration in younger patients $(<75$ years $)$ at low surgical risk and SAVR (if feasible) is therefore the preferred treatment option. Conversely, durability is a lower priority in older patients ( $\geq 75$ years), or those who are inoperable or high risk for surgery, and TAVI is preferred in these groups (particularly if feasible via transfemoral approach). The Heart Team should make tailored recommendations for remaining patients based upon their individual characteristics (Table 6). This guidance should be re-addressed when further data concerning the long-term durability of TAVI become available.

Balloon aortic valvuloplasty (BAV) may be considered as a bridge to TAVI or SAVR in patients with decompensated aortic stenosis and (when feasible) in those with severe aortic stenosis who require urgent high-risk non-cardiac surgery (NCS) (section 12). The procedure carries significant risk of complications ${ }^{234}$ and should only be undertaken after Heart Team discussion.

\subsection{MEDICAL THERAPY}

No medical therapies influence the natural history of aortic stenosis. Statins (which demonstrated favourable effects in preclinical studies) do not affect disease progression ${ }^{246}$ and clinical trials targeting calcium metabolic pathways are ongoing. Patients with heart failure who are unsuitable (or waiting) for SAVR or TAVI should be medically treated according to ESC heart failure Guidelines. ${ }^{247}$ ACEI are safe in aortic stenosis (provided that BP is monitored carefully) and may have beneficial myocardial effects before the onset of symptoms, and after TAVI and SAVR. ${ }^{248-250}$ Coexisting hypertension should be treated to avoid additional afterload, although medication (particularly vasodilators) should be titrated to avoid symptomatic hypotension.

Antithrombotic therapy after TAVI is discussed in section 11.

\subsection{SERIAL TESTING}

Rate of progression of aortic stenosis varies widely. Asymptomatic patients, their family and medical carers need careful education, with emphasis on the importance of regular follow-up (ideally in a Heart Valve Clinic $^{9}$ ) and prompt reporting of symptoms. Those with severe aortic stenosis should be followed up every 6 months (at least) to allow earliest symptom detection (using exercise testing if symptoms are doubtful) and any change in echocardiographic parameters (particularly LVEF). Measurement of natriuretic peptides may be considered.

Several studies suggest that the prognosis of moderate degenerative aortic stenosis is worse than previously considered ${ }^{251-254}$ (particularly if there is significant valve calcification) and these patients should be re-evaluated at least annually. Younger patients with mild aortic stenosis and no significant calcification may be followed up every 2-3 years.

\subsection{SPECIAL PATIENT POPULATIONS}

Women with aortic stenosis have higher mortality than men, resulting from late diagnosis and initial specialist assessment followed by less frequent and delayed referral for intervention. ${ }^{255-257}$ Measures are needed to improve this situation and ensure that both sexes receive equivalent care.

CAD and aortic stenosis frequently coexist and the combination confers higher risk of clinical events, therefore the need to consider revascularization in conjunction with aortic valve intervention is common. The impact of coronary revascularization in patients with silent CAD accompanying aortic stenosis is unclear and further studies are warranted in this context (section 3). Both simultaneous SAVR and CABG, and SAVR late after CABG, carry a higher procedural risk than isolated SAVR. Nevertheless, retrospective data indicate that patients with moderate aortic stenosis, in whom CABG is indicated, benefit from concomitant SAVR. Patients aged $<70$ years with mean gradient progression $\geq 5 \mathrm{mmHg} /$ year benefit from SAVR at the time of CABG once baseline peak gradient exceeds $30 \mathrm{mmHg} .{ }^{258}$ Decisions for individual patients should take into account haemodynamic data, rate of progression, extent of leaflet calcification, life expectancy, and associated comorbidities, as well as the individual risk of concomitant SAVR or deferred TAVI. ${ }^{244}$

Percutaneous coronary intervention (PCI) and TAVI may be undertaken as combined or staged procedures according to the clinical situation, pattern of CAD, and extent of myocardium at risk. ${ }^{259}$ In the SURTAVI trial, there was no significant difference in the primary endpoint (all-cause mortality or stroke at 2-year follow-up) in intermediate-risk patients with severe aortic stenosis and non-complex CAD (SYNTAX score <22) undergoing either TAVI and PCI or SAVR and CABG $[16.0 \%$ (95\% CI, 11.1-22.9) vs. $14 \%$ (95\% CI, 9.2-21.1); $\mathrm{P}=0.62] .{ }^{260}$ Assessing the clinical value of systematic PCI in TAVI patients with significant associated CAD is the objective of ongoing RCTs. Patients with severe symptomatic aortic stenosis and diffuse CAD unsuitable for revascularization should receive 
optimal medical therapy and undergo SAVR or TAVI according to individual characteristics.

Severity of mitral regurgitation accompanying severe aortic stenosis may be overestimated as a result of elevated LV pressures and careful quantification is required. In patients with severe primary mitral regurgitation (PMR), mitral valve surgery is required at the time of SAVR. In patients with severe SMR, surgery may also be considered in the presence of significant annular dilatation and marked LV enlargement. In high-risk or inoperable patients with severe aortic stenosis and severe mitral regurgitation, combined (or more often sequential) TAVI and TEER may be feasible, but there is insufficient experience to allow robust recommendations. ${ }^{261-263}$ In patients with severe PMR, TEER should be considered early if the patient remains symptomatic and mitral regurgitation is still severe after TAVI. In patients with severe SMR, TAVI should be followed by careful clinical and echocardiographic reassessment to determine whether further mitral intervention is required. ${ }^{264}$

Section 4 provides recommendations for the management of aneurysm/dilatation of the ascending aorta accompanying aortic stenosis. Assessment and management of congenital aortic stenosis is addressed in ESC Guidelines on adult congenital heart disease. ${ }^{265}$

\section{Mitral regurgitation}

Mitral regurgitation is the second-most frequent VHD in Europe. ${ }^{1,3}$ The underlying mechanism (primary or secondary) determines the therapeutic approach.

\subsection{PRIMARY MITRAL REGURGITATION}

Primary lesion of one or more components of the mitral valve apparatus characterizes PMR. Degenerative aetiology (fibroelastic deficiency and Barlow disease) is most frequent in Western countries. ${ }^{1,2,266}$ In low-income countries, rheumatic aetiology is the most frequent cause of mitral regurgitation. ${ }^{267}$ Endocarditis can cause PMR and is addressed in the corresponding ESC Guidelines. ${ }^{4}$

\subsubsection{EVALUATION}

Echocardiography is the first choice of imaging technique to grade PMR (Table 7). An integrative approach including qualitative, semi-quantitative, and quantitative measures of mitral regurgitation (besides quantification of LV and LA dimensions) is recommended. ${ }^{24,268}$ Routinely measured effective regurgitant orifice area (EROA) is strongly associated with all-cause mortality, and compared with the general population an excess mortality appears for an EROA $\geq 20 \mathrm{~mm}^{2}$ and steadily increases beyond $40 \mathrm{~mm}^{2} .{ }^{269}$ Evaluation of the specific lesion leading to mitral regurgitation has prognostic implications ${ }^{266,270}$ and is fundamental to determine

Table 7. Severe mitral regurgitation criteria based on 2D echocardiography.

\begin{tabular}{|c|c|c|}
\hline & Primary mitral regurgitation & Secondary mitral regurgitation \\
\hline \multicolumn{3}{|l|}{ Qualitative } \\
\hline Mitral valve morphology & $\begin{array}{l}\text { Flail leaflet, ruptured papillary muscle, } \\
\text { severe retraction, large perforation }\end{array}$ & Normal leaflets but with severe tenting, poor leaflet coaptation \\
\hline Colour flow jet area & $\begin{array}{l}\text { Large central jet ( }>50 \% \text { of } L A \text { ) or eccentric } \\
\text { wall impinging jet of variable size }\end{array}$ & $\begin{array}{l}\text { Large central jet ( }>50 \% \text { of } L A \text { ) or eccentric wall impinging jet } \\
\text { of variable size }\end{array}$ \\
\hline Flow convergence & Large throughout systole & Large throughout systole \\
\hline Continuous wave Doppler jet & Holosystolic/dense/triangular & Holosystolic/dense/triangular \\
\hline \multicolumn{3}{|l|}{ Semiquantitative } \\
\hline Vena contracta width (mm) & $\geq 7$ ( $\geq 8 \mathrm{~mm}$ for biplane) & $\geq 7$ ( $\geq 8 \mathrm{~mm}$ for biplane) \\
\hline Pulmonary vein flow & Systolic flow reversal & Systolic flow reversal \\
\hline Mitral inflow & E-wave dominant $(>1.2 \mathrm{~m} / \mathrm{s})$ & E-wave dominant $(>1.2 \mathrm{~m} / \mathrm{s})$ \\
\hline TVI mitral/TVI aortic & $>1.4$ & $>1.4$ \\
\hline \multicolumn{3}{|l|}{ Quantitative } \\
\hline EROA (2D PISA, mm²) & $\geq 40 \mathrm{~mm}^{2}$ & $\geq 40 \mathrm{~mm}^{2}$ (may be $\geq 30 \mathrm{~mm}^{2}$ if elliptical regurgitant orifice area) \\
\hline Regurgitant volume (mL/beat) & $\geq 60 \mathrm{~mL}$ & $\geq 60 \mathrm{~mL}$ (may be $\geq 45 \mathrm{~mL}$ if low flow conditions) \\
\hline Regurgitant fraction (\%) & $\geq 50 \%$ & $\geq 50 \%$ \\
\hline \multicolumn{3}{|l|}{ Structural } \\
\hline Left ventricle & Dilated (ESD $\geq 40 \mathrm{~mm})$ & Dilated \\
\hline Left atrium & $\begin{array}{l}\text { Dilated (diameter } \geq 55 \mathrm{~mm} \text { or volume } \\
\geq 60 \mathrm{~mL} / \mathrm{m}^{2} \text { ) }\end{array}$ & Dilated \\
\hline \multicolumn{3}{|c|}{$\begin{array}{l}\text { 2D: two-dimensional; ESD: endsystolic diameter; EROA: effective regurgitant orifice area; LA: left atrium; PMR: primary mitral regurgitation; } \\
\text { SMR: secondary mitral regurgitation; PISA: proximal isovelocity surface area; TVI: time-velocity integral. Adapted from Lancellotti P et al. } \\
\text { Recommendations for the echocardiographic assessment of native valvular regurgitation: an executive summary from the European Association of } \\
\text { Cardiovascular Imaging. Eur Heart J Cardiovasc Imaging 2013;14:611-644. Copyright (2013) by permission of Oxford University Press on behalf of the } \\
\text { European Society of Cardiology. Reproduced from Zoghbi WA et al. Recommendations for noninvasive evaluation of native valvular regurgitation: a report } \\
\text { from the American Society of Echocardiography developed in collaboration with the Society for Cardiovascular Magnetic Resonance. J Am Soc } \\
\text { Echocardiogr 2017;30:303-371. Copyright (2017), with permission from the American Society of Echocardiography. }\end{array}$} \\
\hline
\end{tabular}


the feasibility of surgical and transcatheter valve repair ${ }^{271-273}$ (Supplementary Figure 1). Three-dimensional TOE provides an 'en face' view of the mitral leaflets resembling the surgical inspection of the valve, thereby facilitating Heart Team discussion..$^{24,268}$ In addition, 3D echocardiography has shown better agreement with CMR in quantifying the regurgitant volume than $2 \mathrm{D}$ echocardiography, particularly in eccentric, multiple and late-systolic regurgitant jets. ${ }^{274-277}$ When various echocardiographic parameters used to grade mitral regurgitation are inconsistent, CMR is a valid alternative to quantify the regurgitant volume and is the reference standard to quantify LV and LA volumes. ${ }^{278}$ In addition, quantification of mitral regurgitation with $\mathrm{CMR}$ has shown prognostic implications. ${ }^{277}$ Finally, preliminary data show that myocardial fibrosis assessed with CMR is frequent in PMR and has been associated with sudden cardiac death and ventricular arrhythmias. ${ }^{279}$

Exercise echocardiography permits evaluation of changes in mitral regurgitant volume and pulmonary pressures during peak exercise and is particularly helpful in patients with discordant symptoms and regurgitation grade at rest. ${ }^{280,281}$ In asymptomatic patients with severe PMR and non-dilated LV and LA, low BNP values are associated with low mortality and can be useful during follow-up. ${ }^{41,282}$

LV dimensions and ejection fraction are considered to guide the management of patients with severe PMR. However, there is cumulative evidence showing that LV global longitudinal strain has incremental prognostic value in patients treated with surgical repair. ${ }^{283,284}$ Recently, the Mitral Regurgitation International Database (MIDA) score has been proposed to estimate the risk of all-cause mortality in patients with severe PMR due to flail leaflet, who are under medical treatment or surgically treated. ${ }^{285}$ Among the variables included in the score, LA diameter $\geq 55 \mathrm{~mm}$ and LVESD $\geq 40 \mathrm{~mm}$ are new thresholds that have been included in the current recommendations.

Right heart catheterization is systematically used to confirm pulmonary hypertension diagnosed by echocardiography when this is the only criterion to refer the patient for surgery.

\subsubsection{INDICATIONS FOR INTERVENTION}

Urgent surgery is indicated in patients with acute severe mitral regurgitation. In the case of papillary muscle rupture as the underlying disease, valve replacement is generally required.

Indications for surgery in severe chronic PMR are shown in the following table of recommendations and in Figure 5. Surgery is recommended in patients with symptomatic severe PMR and acceptable surgical risk according to the Heart Team decision. The presence of $\mathrm{LVEF} \leq 60 \%$, LVESD $\geq 40 \mathrm{~mm},{ }^{285,286}$ LA volume $\geq 60 \mathrm{~mL} / \mathrm{m}^{2}$ or diameter $\geq 55 \mathrm{~mm},{ }^{287,288}$ systolic pulmonary arterial pressure $(\mathrm{SPAP})>50 \mathrm{mmHg},{ }^{289}$ and $\mathrm{AF}^{290,291}$ have been associated with worse outcomes and are considered triggers for intervention regardless of symptomatic status. ${ }^{292}$ In the absence of these criteria, watchful waiting is a safe strategy in asymptomatic patients with severe PMR and ideally should be performed in a Heart Valve Clinic.

When surgery is considered, mitral valve repair is the surgical intervention of first choice when the results are expected

\section{Recommendations on indications for intervention in severe primary mitral regurgitation}

\begin{tabular}{|c|c|c|}
\hline Recommendations & Class $^{a}$ & Levelb $^{b}$ \\
\hline $\begin{array}{l}\text { Mitral valve repair is the recommended surgical } \\
\text { technique when the results are expected to be } \\
\text { durable. }{ }^{293-296}\end{array}$ & I & B \\
\hline $\begin{array}{l}\text { Surgery is recommended in symptomatic patients } \\
\text { who are operable and not high risk. }{ }^{293-296}\end{array}$ & I & B \\
\hline $\begin{array}{l}\text { Surgery is recommended in asymptomatic patients } \\
\text { with LV dysfunction (LVESD } \geq 40 \mathrm{~mm} \text { and/or LVEF } \\
\leq 60 \%)^{277,286,292}\end{array}$ & I & B \\
\hline $\begin{array}{l}\text { Surgery should be considered in asymptomatic } \\
\text { patients with preserved LV function (LVESD }<40 \mathrm{~mm} \\
\text { and LVEF }>60 \% \text { ) and AF secondary to mitral } \\
\text { regurgitation or pulmonary hypertensionc (SPAP at } \\
\text { rest }>50 \mathrm{mmHg} \text { ). }{ }^{285,289}\end{array}$ & Ila & B \\
\hline $\begin{array}{l}\text { Surgical mitral valve repair should be considered in } \\
\text { low-risk asymptomatic patients with LVEF }>60 \% \text {, } \\
\text { LVESD }<40 \mathrm{mmd} \text { and significant LA dilatation } \\
\text { (volume index } \geq 60 \mathrm{~mL} / \mathrm{m}^{2} \text { or diameter } \geq 55 \mathrm{~mm} \text { ) } \\
\text { when performed in a Heart Valve Centre and } \\
\text { a durable repair is likely. }\end{array}$ & Ila & B \\
\hline $\begin{array}{l}\text { TEER may be considered in symptomatic patients } \\
\text { who fulfil the echocardiographic criteria of eligibility, } \\
\text { are judged inoperable or at high surgical risk by the } \\
\text { Heart Team and for whom the procedure is not } \\
\text { considered futile. } .^{299-302}\end{array}$ & Illb & B \\
\hline
\end{tabular}

AF: atrial fibrillation; LA: left atrium/left atrial; LV: left ventricle/left ventricular; LVEF: left ventricular ejection fraction; LVESD: left ventricular end-systolic diameter; SPAP: systolic pulmonary arterial pressure; TEER: transcatheter edge-to-edge repair. ${ }^{a}$ Class of recommendations. 'bevel of evidence. cIf an elevated SPAP is the only indication for surgery, the value should be confirmed by invasive measurement. ${ }^{\mathrm{C}}$ Cut-offs refer to average-size adults and may require adaptations in patients with unusually small or large stature.

to be durable according to the Heart Team evaluation since it is associated with better survival compared to mitral valve replacement. ${ }^{293,294}$ PMR due to segmental valve prolapse can be repaired with a low risk of recurrence and reoperation. ${ }^{294-296}$ The reparability of rheumatic lesions, extensive valve prolapse and to a greater extent leaflet calcification or extensive annular calcification is more challenging. ${ }^{297,298}$ Patients requiring a predictably complex repair should undergo surgery in experienced repair centres with high repair rates, low operative mortality, and a record of durable results. When repair is not feasible, mitral valve replacement with preservation of the subvalvular apparatus is favoured.

Transcatheter mitral valve implantation for severe PMR is a safe alternative in patients with contraindications for surgery or high operative risk. ${ }^{299-302}$ TEER is the most evidenced, while the safety and efficacy of other techniques have been demonstrated in smaller series. ${ }^{303-306}$ The efficacy of more recent TEER system iterations $^{307}$ will be investigated in high-risk (MITRA-HR study NCT03271762) 308 $^{3}$ and intermediate-risk patients (REPAIR-MR study NCT04198870).

\subsubsection{MEDICAL THERAPY}

In acute mitral regurgitation, nitrates and diuretics are used to reduce filling pressures. Sodium nitroprusside reduces afterload 


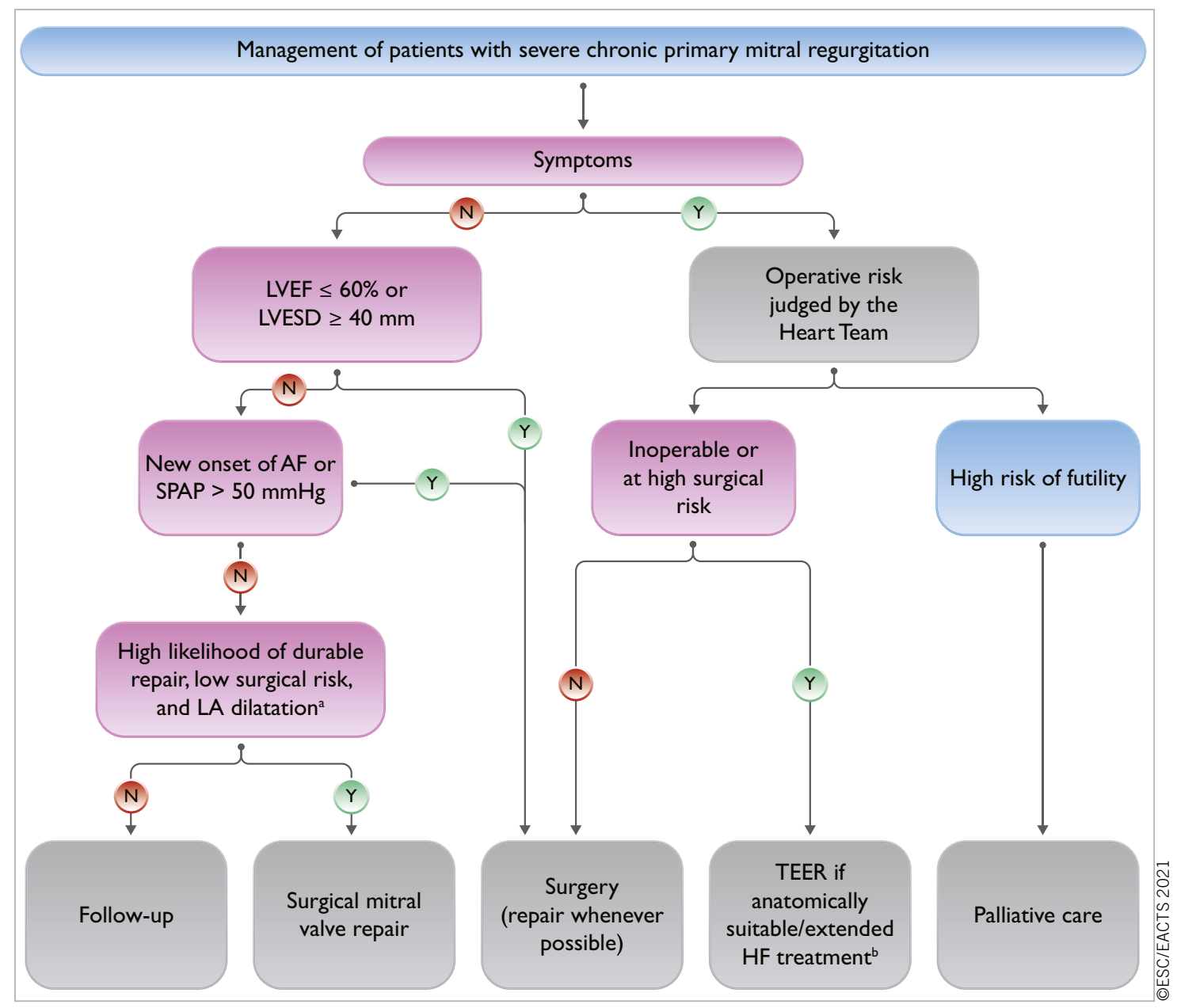

Figure 5. Management of patients with severe chronic primary mitral regurgitation. AF: atrial fibrillation; HF: heart failure; LA: left atrium/ left atrial; LVEF: left ventricular ejection fraction; LVESD: left ventricular end-systolic diameter; SPAP: systolic pulmonary arterial pressure; TEER: transcatheter edge-to-edge repair. ${ }^{a} L A$ dilatation: volume index $\geq 60 \mathrm{~mL} / \mathrm{m}^{2}$ or diameter $\geq 55 \mathrm{~mm}$ at sinus rhythm. ${ }^{b}$ Extended heart failure treatment includes the following: CRT; ventricular assist devices; heart transplantation. ${ }^{247}$

and regurgitant fraction. Inotropic agents and an intra-aortic balloon pump are of use in hypotension and haemodynamic instability.

In chronic PMR with preserved LVEF, there is no evidence to support the prophylactic use of vasodilators. In patients with overt heart failure, medical treatment as per current heart failure guidelines applies. ${ }^{247}$

\subsubsection{SERIAL TESTING}

Asymptomatic patients with severe mitral regurgitation and LVEF $>60 \%$ should be followed clinically and by echocardiography every 6 months, ideally in the setting of a Heart Valve Centre. ${ }^{309}$ Measurement of BNP levels, exercise echocardiography, electrocardiogram-Holter monitoring and CMR are useful complementary diagnostic and risk stratification tools. ${ }^{268}$ The association between PMR, sudden cardiac death and ventricular arrhythmias remains controversial. ${ }^{310-312}$ The presence of mitral annulus disjunction (abnormal atrial displacement of the hinge point of the mitral valve away from the ventricular myocardium) has been also associated with increased risk of ventricular arrhythmias. ${ }^{310,311,313}$ Interestingly, the majority of these patients did not have severe mitral regurgitation. In asymptomatic patients with severe PMR and progressive increase of LV size (LVESD approaching $40 \mathrm{~mm}$ ) or decrease of LVEF on serial studies, surgical mitral valve repair should be discussed. Asymptomatic patients with moderate mitral regurgitation and preserved LV function can be followed on a yearly basis and echocardiography should be performed every 1-2 years. After intervention, serial follow-up focuses on evaluation of symptomatic status, presence of arrhythmic events, assessment of valve function, ${ }^{314}$ and recurrence of mitral regurgitation. After surgical mitral valve repair, high-volume centres have reported good durability with a recurrence rate of moderate or severe mitral regurgitation of $12.5 \%$ at 20 years of follow-up. ${ }^{296}$ After transcatheter mitral valve repair, the currently reported rates of residual moderate and severe mitral regurgitation (23-30\%) would suggest that yearly echocardiogram is appropriate. . $^{14,300,301}$

\subsubsection{SPECIAL POPULATIONS}

Sex differences in terms of prevalence of underlying aetiology of PMR and management have been reported. ${ }^{298,315,316}$ Despite the 
reduction in the prevalence of rheumatic disease in Western countries, women still have higher rates of rheumatic mitral regurgitation than men and emerging aetiologies such as radiation heart disease are also more frequent in women. ${ }^{297}$ These aetiologies are often characterized by severe calcification of the mitral valve apparatus and associated with mitral stenosis precluding durable repair. Women with PMR referred for surgical treatment received mitral valve repair at a similar rate to men. ${ }^{316}$ However, women more frequently present with post-operative heart failure, probably related to a later referral and more advanced disease as compared to men.

\subsection{SECONDARY MITRAL REGURGITATION}

In SMR, the valve leaflets and chordae are structurally normal and mitral regurgitation results from an imbalance between closing and tethering forces secondary to alterations in LV and LA geometry. ${ }^{317,318}$ It is most commonly seen in dilated or ischaemic cardiomyopathies, both in severely dilated LV with markedly depressed LV function or after an isolated infero-basal myocardial infarction leading to posterior leaflet tethering, despite almost normal LV size and ejection fraction. SMR may also arise as a consequence of LA enlargement and mitral annular dilatation in patients with longstanding AF, in whom LVEF is usually normal and LV dilatation less pronounced (so called 'atrial functional mitral regurgitation'). ${ }^{319}$

\subsubsection{EVALUATION}

The echocardiographic criteria to define severe SMR do not differ from those used in PMR and an integrative approach should be used (Table 7). ${ }^{24,268}$ However, it should be acknowledged that when quantifying EROA and regurgitant volume in SMR, lower thresholds may be applied to define severe SMR. In heart failure patients, the total forward LV stroke volume is lower and that may lead to lower estimated regurgitant volume $(<60 \mathrm{~mL} /$ beat $)$. Calculation of regurgitant fraction in those circumstances could account for lower flows and has shown prognostic implications. ${ }^{320}$ In addition, the crescent shape of the regurgitant orifice, characteristic of SMR, may lead to underestimation of the vena contracta width and of the EROA. An EROA $\geq 30 \mathrm{~mm}^{2}$ by $2 \mathrm{D}$ proximal isovelocity surface area (PISA) likely corresponds to severe SMR. In contrast, whether an EROA $\geq 20 \mathrm{~mm}^{2}$ defines severe SMR remains controversial. In heart failure patients, even mild mitral regurgitation is associated with poor prognosis ${ }^{321}$ and evidence that surgical or transcatheter treatment of moderate SMR does not seem to improve patient outcomes ${ }^{322,323}$ supports the change in definition of severe SMR. Caution is required, therefore, when labelling severe SMR based solely on prognostic implications. Other factors such as the extent of myocardial scar, as assessed with CMR, have been associated with poor prognosis. ${ }^{324}$ In addition, LVEF has been shown to be misleading in patients with severe SMR, while LV global longitudinal strain has been shown to have incremental prognostic value. ${ }^{325,326}$ The use of $3 \mathrm{D}$ echocardiography, CMR and exercise echocardiography may help to identify patients with severe mitral regurgitation when 2D echocardiography at rest is inconclusive. ${ }^{24,268}$

\subsubsection{MEDICAL THERAPY}

Optimal medical therapy in line with the guidelines for the management of heart failure ${ }^{247}$ should be the first and essential step in the management of all patients with SMR and should include replacement of ACEI or ARB with sacubitril/valsartan, sodiumglucose co-transporter 2 inhibitors and/or ivabradine, whenever indicated. ${ }^{247,327}$ Indications for cardiac resynchronization therapy (CRT) should be evaluated in accordance with related guidelines. ${ }^{247}$ If symptoms persist after optimization of conventional heart failure therapy, options for mitral valve intervention should be promptly evaluated before further deterioration of LV systolic function or cardiac remodelling occur.

\subsubsection{INDICATIONS FOR INTERVENTION}

Chronic SMR is associated with impaired prognosis ${ }^{321,328}$ and its interventional management is complex (see recommendations on indications for mitral valve intervention in chronic severe SMR, and Figure 6). The detailed analysis of the available level of evidence made by the methodology group of the task force is available in Supplementary Section 5. The importance of decision making by a multidisciplinary Heart Team needs to be emphasized in this setting. The Heart Team, including a heart failure specialist, should optimize guideline-directed medical therapy (GDMT) and consider the indications of electrophysiological, transcatheter and surgical interventions, their priority and order of implementation.

The evidence supporting surgical intervention remains limited. Mitral valve surgery is recommended in patients with severe SMR undergoing CABG or other cardiac surgery. ${ }^{329,330}$ The surgical approach has to be tailored to the individual patient. ${ }^{247,331}$ In selected patients without advanced LV remodelling, mitral valve repair with an undersized complete rigid ring restores valve competence, improves symptoms, and results in reverse LV remodelling. ${ }^{331}$ Additional valvular/subvalvular techniques or chordal sparing valve replacement may be considered in patients with echocardiographic predictors of repair failure. ${ }^{332}$ Valve replacement avoids recurrence of mitral regurgitation, although this does not translate into better LV reverse remodelling or survival. ${ }^{333}$ Indications for isolated mitral valve surgery in SMR are particularly restrictive, owing to significant procedural risk, high rates of recurrent mitral regurgitation, and the absence of proven survival benefit. ${ }^{333-335}$ In patients with atrial functional mitral regurgitation, LVEF is usually normal, LV dilatation less pronounced and mitral annular dilatation represents the main mechanism of mitral regurgitation. This subgroup may be more effectively treated by ring annuloplasty often associated with ablation of AF but evidence is still limited. ${ }^{319}$

TEER with the MitraClip system is a minimal-invasive treatment option for SMR. Two RCTs (COAPT and MITRA-FR) 323,336,337 have evaluated its safety and efficacy in patients with symptomatic heart failure and severe SMR persisting despite medical therapy, who were considered either ineligible or not appropriate for surgery by the Heart Team (Supplementary Table 7). The results indicate that the procedure is safe and effectively reduces SMR up to 3 years. ${ }^{338}$ However, in the MITRA-FR trial, ${ }^{323,336}$ MitraClip implantation had no impact on the primary endpoint of all-cause 


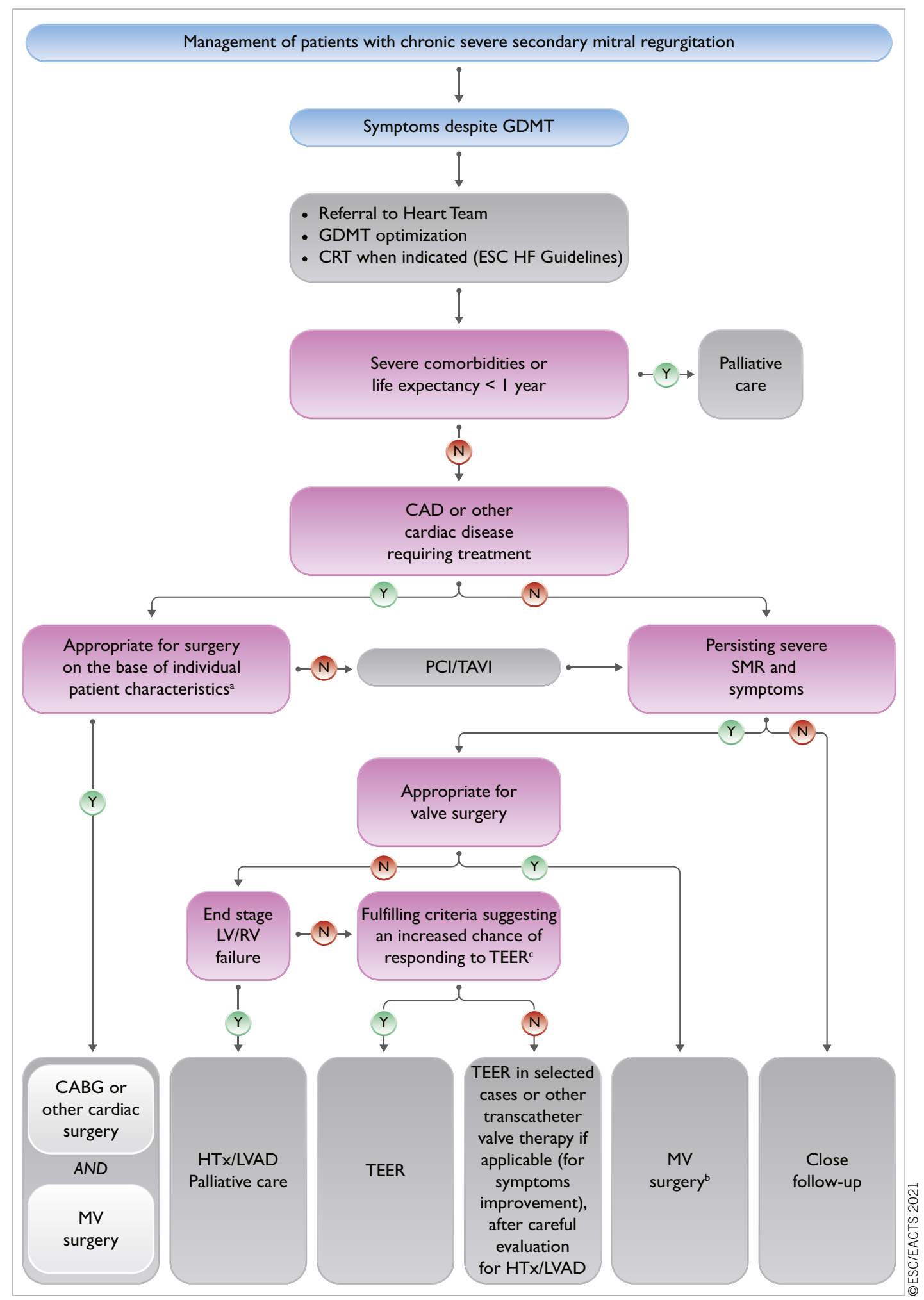

Figure 6. Management of patients with chronic severe secondary mitral regurgitation. CAD: coronary artery disease; CABG: coronary artery bypass grafting; CRT: cardiac resynchronization therapy; ESC: European Society of Cardiology; GDMT: guideline-directed medical therapy; HF: heart failure; HTx: heart transplantation; LVAD: left ventricular assist devices; LV: left ventricle/left ventricular; LVEF: left ventricular ejection fraction; $M V$ : mitral valve; PCI: percutaneous coronary intervention; RV: right ventricle/right ventricular; SMR: secondary mitral regurgitation; TAVI: transcatheter aortic valve implantation; TEER: transcatheter edge-to-edge repair. ${ }^{a} \mathrm{LVEF}$, predicted surgical risk, amount of myocardial viability, coronary anatomy/target vessels, type of concomitant procedure needed, TEER eligibility, likelihood of durable surgical repair, need of surgical mitral replacement, local expertise. ${ }^{b}$ Particularly when concomitant tricuspid valve surgery is needed. ${ }^{c}$ COAPT criteria (Cardiovascular Outcomes Assessment of the MitraClip Percutaneous Therapy for Heart Failure Patients With Functional Mitral Regurgitation): see Supplementary Table 7. 
mortality or heart failure hospitalization at 12 months and 2 years compared to GDMT alone. In the COAPT trial, ${ }^{337}$ MitraClip implantation substantially reduced the primary endpoint of cumulative hospitalizations for heart failure, as well as several pre-specified secondary endpoints, including all-cause mortality at 2 years.

Subanalyses of the COAPT trial confirm the positive response to TEER in several patient subgroups; ${ }^{339-343}$ conversely, the effect of the interventional treatment was neutral throughout all subgroups in an echocardiographic subanalyses of the MITRA-FR trial. ${ }^{344}$

The conflicting results of these two trials have generated considerable discussion. These diverging results might be partially explained by effect size of the trials, differences in trial design,

\section{Recommendations on indications for mitral valve intervention in chronic severe secondary mitral regurgitation ${ }^{a}$}

\begin{tabular}{|c|c|c|}
\hline Recommendations & Class $^{b}$ & Level $^{c}$ \\
\hline $\begin{array}{l}\text { Valve surgery/intervention is recommended only } \\
\text { in patients with severe SMR who remain } \\
\text { symptomatic despite GDMT (including CRT if } \\
\text { indicated) and has to be decided by a structured } \\
\text { collaborative Heart Team. }{ }^{247,323,336,337}\end{array}$ & I & B \\
\hline $\begin{array}{l}\text { Patients with concomitant coronary artery or other } \\
\text { cardiac disease requiring treatment }\end{array}$ & & \\
\hline $\begin{array}{l}\text { Valve surgery is recommended in patients } \\
\text { undergoing CABG or other cardiac } \\
\text { surgery. }\end{array}$ & I & B \\
\hline $\begin{array}{l}\text { In symptomatic patients, who are judged not } \\
\text { appropriate for surgery by the Heart Team on the } \\
\text { basis of their individual characteristics, }{ }^{d} \mathrm{PCI} \\
\text { (and/or TAVI) possibly followed by TEER (in case } \\
\text { of persisting severe SMR) should be considered. }\end{array}$ & Ila & C \\
\hline \multicolumn{3}{|c|}{$\begin{array}{l}\text { Patients without concomitant coronary artery or other cardiac } \\
\text { disease requiring treatment }\end{array}$} \\
\hline $\begin{array}{l}\text { TEER should be considered in selected } \\
\text { symptomatic patients, not eligible for surgery and } \\
\text { fulfilling criteria suggesting an increased chance } \\
\text { of responding to the treatment. }{ }^{337,338,356,357 \mathrm{e}}\end{array}$ & Ila & B \\
\hline $\begin{array}{l}\text { Valve surgery may be considered in symptomatic } \\
\text { patients judged appropriate for surgery by the } \\
\text { Heart Team. }\end{array}$ & Ilb & C \\
\hline $\begin{array}{l}\text { In high-risk symptomatic patients not eligible for } \\
\text { surgery and not fulfilling the criteria suggesting } \\
\text { an increased chance of responding to TEER, the } \\
\text { Heart Team may consider in selected cases } \\
\text { a TEER procedure or other transcatheter valve } \\
\text { therapy if applicable, after careful evaluation for } \\
\text { ventricular assist device or heart transplant.e }\end{array}$ & Ilb & C \\
\hline
\end{tabular}

2D: two-dimensional; CABG: coronary artery bypass grafting. CRT: cardiac resynchronization therapy; EROA: effective regurgitation orifice area; GDMT: guideline-directed medical therapy; LVEF: left ventricular ejection fraction; SMR: secondary mitral regurgitation, $\mathrm{PCl}$ : percutaneous coronary intervention; SMR: secondary mitral regurgitation; TAVI: transcatheter aortic valve implantation;

TEER: transcatheter edge-to-edge repair. aSee Table 7 for SMR quantification (an EROA $\geq 30 \mathrm{~mm}^{2}$ by $2 \mathrm{D}$ proximal isovelocity surface area corresponds likely to severe SMR). Quantification of SMR must always be performed under optimal guidelines-directed medical treatment. ${ }^{b}$ Class of recommendation. 'Level of evidence. ${ }^{\mathrm{d}} \mathrm{LVEF}$, predicted surgical risk, amount of myocardial viability, coronary anatomy/target vessels, type of concomitant procedure needed, TEER eligibility, likelihood of durable surgical repair, need of surgical mitral replacement, local expertise. ${ }^{\text {eCOAPT }}$ criteria (Cardiovascular Outcomes Assessment of the MitraClip Percutaneous Therapy for Heart Failure Patients With Functional Mitral Regurgitation): see Supplementary Table 7. patient selection, echocardiographic assessment of SMR severity, use of medical therapy, and technical factors. Patients in COAPT demonstrated greater severity of SMR (EROA $41 \pm 15 \mathrm{~mm}^{2}$ vs. $31 \pm 10 \mathrm{~mm}^{2}$ ) and less LV dilatation (mean indexed LV enddiastolic volume $101 \pm 34 \mathrm{~mL} / \mathrm{m}^{2}$ vs. $135 \pm 35 \mathrm{~mL} / \mathrm{m}^{2}$ ) than those enrolled in MITRA-FR. Perhaps reflecting greater severity of $\mathrm{SMR}$ in relation to $\mathrm{LV}$ dimensions ('disproportionate' mitral regurgitation), patients in COAPT were overall more likely to benefit from TEER in terms of reduced mortality and heart failure hospitalization. ${ }^{345}$

Additional studies are needed to identify patients who will benefit the most from TEER.

Therefore, TEER should be considered in selected patients with severe SMR fulfilling the COAPT inclusion criteria, ${ }^{346-348}$ who receive optimal medical therapy supervised by a heart failure specialist and are as close as possible to the patients actually enrolled in the study. Optimization of the procedural result should also be pursued. In addition, TEER may be considered only in selected cases when the COAPT criteria are not fulfilled with the aim of improving symptoms and quality of life. ${ }^{349-353}$ In patients with less severe SMR (EROA $<30 \mathrm{~mm}^{2}$ ) and advanced LV dilatation/dysfunction, the prognostic benefit of MitraClip remains unproven. ${ }^{323,354,355}$ Patients with end-stage LV and/or RV failure and no option for revascularization may be better served by cardiac transplantation or LV assist device implantation. Valve intervention is generally not an option when LVEF is $<15 \%{ }^{247}$

The management of moderate ischaemic SMR in patients undergoing $\mathrm{CABG}$ remains an object of debate. ${ }^{322,330}$ Surgery is more likely to be considered if myocardial viability is present and if comorbidity is low. Exercise-induced dyspnoea and a large increase in mitral regurgitation severity and SPAP favour combined surgery.

Transcatheter mitral valve repair systems other than TEER, as well as transcatheter mitral valve replacement devices, are currently the subject of intense investigation but clinical data are still limited.

\section{Mitral stenosis}

Aetiology of mitral stenosis is mostly rheumatic or degenerative. Rheumatic fever is the most common cause of mitral stenosis worldwide. Its prevalence has greatly decreased in industrialized countries, but it remains a significant healthcare problem in developing countries and affects young patients. ${ }^{2,267,358}$ Degenerative mitral stenosis related to MAC is a distinct pathology and its prevalence significantly increases with age. ${ }^{359,360}$ Both types of mitral stenosis are more frequent in females. ${ }^{361}$ In rare cases, mitral stenosis due to valve rigidity but without commissural fusion, may be related to chest radiation, carcinoid heart disease, or inherited metabolic diseases.

\subsection{RHEUMATIC MITRAL STENOSIS}

\subsubsection{EVALUATION}

Clinically significant mitral stenosis is defined by a mitral valve area (MVA) $\leq 1.5 \mathrm{~cm}^{2}$. Commissural fusion with thickening of 
the posterior leaflet is the most important mechanism of stenosis. Echocardiography is the preferred method for diagnosis, assessment of severity, and haemodynamic consequences of mitral stenosis. Valve area using 2D planimetry is the reference measurement of mitral stenosis severity, whereas mean transvalvular gradient and pulmonary pressures reflect its consequences and have a prognostic role. ${ }^{362}$ 3D-TTE planimetry may have an additional diagnostic value. TTE usually provides sufficient information for routine management. Scoring systems have been developed to help assess suitability for percutaneous mitral commissurotomy (PMC; Supplementary Table 8), ${ }^{363-365}$ TOE should be performed to exclude LA thrombus before PMC or after an embolic episode, and to obtain detailed information on mitral anatomy (commissural zones and subvalvular apparatus) before intervention when TTE is suboptimal. Stress testing is indicated in patients with no symptoms or symptoms equivocal or discordant with the severity of mitral stenosis. Exercise echocardiography may provide objective information by assessing changes in mitral gradient and pulmonary artery pressure and is superior to DSE. Echocardiography plays an important role in the periprocedural monitoring of PMC and follow-up.

\subsubsection{INDICATIONS FOR INTERVENTION}

The type of treatment (PMC or surgery), as well as its timing, should be decided based on clinical characteristics, anatomy of valve and subvalvular apparatus, and local expertise. ${ }^{366-369}$ In general, indication for intervention should be limited to patients with clinically significant (moderate-to-severe) rheumatic mitral stenosis (valve area $\leq 1.5 \mathrm{~cm}^{2}$ ) in whom PMC has had a significant impact on its management. In Western countries where incidence of rheumatic fever and number of PMC is low, this treatment should be restricted to expert operators in specialized centres to improve safety and procedural success rate. ${ }^{366}$ Efforts should be made to increase availability of PMC in developing countries where access to treatment is limited due to economic reasons. ${ }^{267}$ PMC should be considered as an initial treatment for selected patients with mild to moderate calcification or impaired subvalvular apparatus, but who have otherwise favourable clinical characteristics. ${ }^{360}$

The management of clinically significant rheumatic mitral stenosis is summarized in Figure $\mathbf{7}$ and the indications and contraindications for PMC are provided in the table of recommendations below, and Table 8.

\begin{tabular}{|c|c|c|}
\hline \multicolumn{3}{|c|}{$\begin{array}{l}\text { Recommendations on indications for percutaneous mitral } \\
\text { commissurotomy and mitral valve surgery in clinically } \\
\text { significant (moderate or severe) mitral stenosis (valve area } \\
\leq 1.5 \mathrm{~cm}^{2} \text { ) }\end{array}$} \\
\hline Recommendations & Class $^{\mathrm{a}}$ & Level $^{\mathrm{b}}$ \\
\hline $\begin{array}{l}\text { PMC is recommended in symptomatic patients } \\
\text { without unfavourable characteristicse for } \\
\text { PMC. }{ }^{360,363-365,367}\end{array}$ & I & B \\
\hline $\begin{array}{l}\text { PMC is recommended in any symptomatic } \\
\text { patients with a contraindication or a high risk for } \\
\text { surgery. }\end{array}$ & I & C \\
\hline
\end{tabular}

\begin{tabular}{|l|l|l|}
\hline $\begin{array}{l}\text { Mitral valve surgery is recommended in } \\
\text { symptomatic patients who are not suitable for }\end{array}$ & I & C \\
PMC in the absence of futility. & & \\
\hline $\begin{array}{l}\text { PMC should be considered as initial treatment in } \\
\text { symptomatic patients with suboptimal anatomy }\end{array}$ & & \\
but no unfavourable clinical characteristics for & Ila & C \\
PMC. & & \\
\hline PMC should be considered in asymptomatic & & \\
patients without unfavourable clinical and & & \\
anatomical characteristics ${ }^{c}$ for PMC and: & & \\
- High thromboembolic risk (history of systemic & & \\
embolism, dense spontaneous contrast in the & Ila & C \\
LA, new-onset or paroxysmal AF), and/or & & \\
- High risk of haemodynamic decompensation & & \\
(systolic pulmonary pressure $>50$ mmHg at & & \\
rest, need for major NCS, desire for pregnancy). & & \\
\hline
\end{tabular}

AF: atrial fibrillation; LA: left atrium/left atrial; MVA: mitral valve area; NCS: non-cardiac surgery; PMC: percutaneous mitral commissurotomy. ${ }^{a}$ Class of recommendation. ${ }^{b}$ Level of evidence. ${ }^{c}$ Unfavourable characteristics for PMC can be defined by the presence of several of the following characteristics. Clinical characteristics: old age, history of commissurotomy, New York Heart Association class IV, permanent AF, severe pulmonary hypertension. Anatomical characteristics: echocardiographic score $>8$, Cormier score 3 (calcification of mitral valve of any extent as assessed by fluoroscopy), very small MVA, severe tricuspid regurgitation. For the definition of scores, see Supplementary Table 8.

Table 8. Contraindications for percutaneous mitral commissurotomy in rheumatic mitral stenosis ${ }^{a}$.

Contraindications

MVA $>1.5 \mathrm{~cm}^{2}$ a

LA thrombus

More than mild mitral regurgitation

Severe or bi-commissural calcification

Absence of commissural fusion

Severe concomitant aortic valve disease, or severe combined tricuspid stenosis and regurgitation requiring surgery

Concomitant $C A D$ requiring bypass surgery

CAD: coronary artery disease; LA: left atrium/left atrial; MVA: mitral valve area; PMC: percutaneous mitral commissurotomy. ${ }^{a}$ PMC may be considered in patients with valve area $>1.5 \mathrm{~cm}^{2}$ with symptoms that cannot be explained by another cause and if the anatomy is favourable.

\subsubsection{MEDICAL THERAPY}

Diuretics, beta-blockers, digoxin, non-dihydropyridine calcium channel blockers and ivabradine can improve symptoms. Anticoagulation with vitamin $\mathrm{K}$ antagonist (VKA) with a target international normalized ratio (INR) between 2 and 3 is indicated in patients with AF. Patients with moderate-to-severe mitral stenosis and AF should be kept on VKA and not receive NOACs. Currently there is no solid evidence to support the use of NOACs in this setting ${ }^{370}$ and a randomized clinical trial is underway (INVICTUS VKA NCT 02832544). Neither cardioversion nor catheter pulmonary vein isolation are indicated before intervention in patients with significant mitral stenosis, as they do not durably restore sinus rhythm. If AF is of recent onset and the LA is only moderately enlarged, cardioversion should be performed soon after successful intervention, it should also be considered in patients 


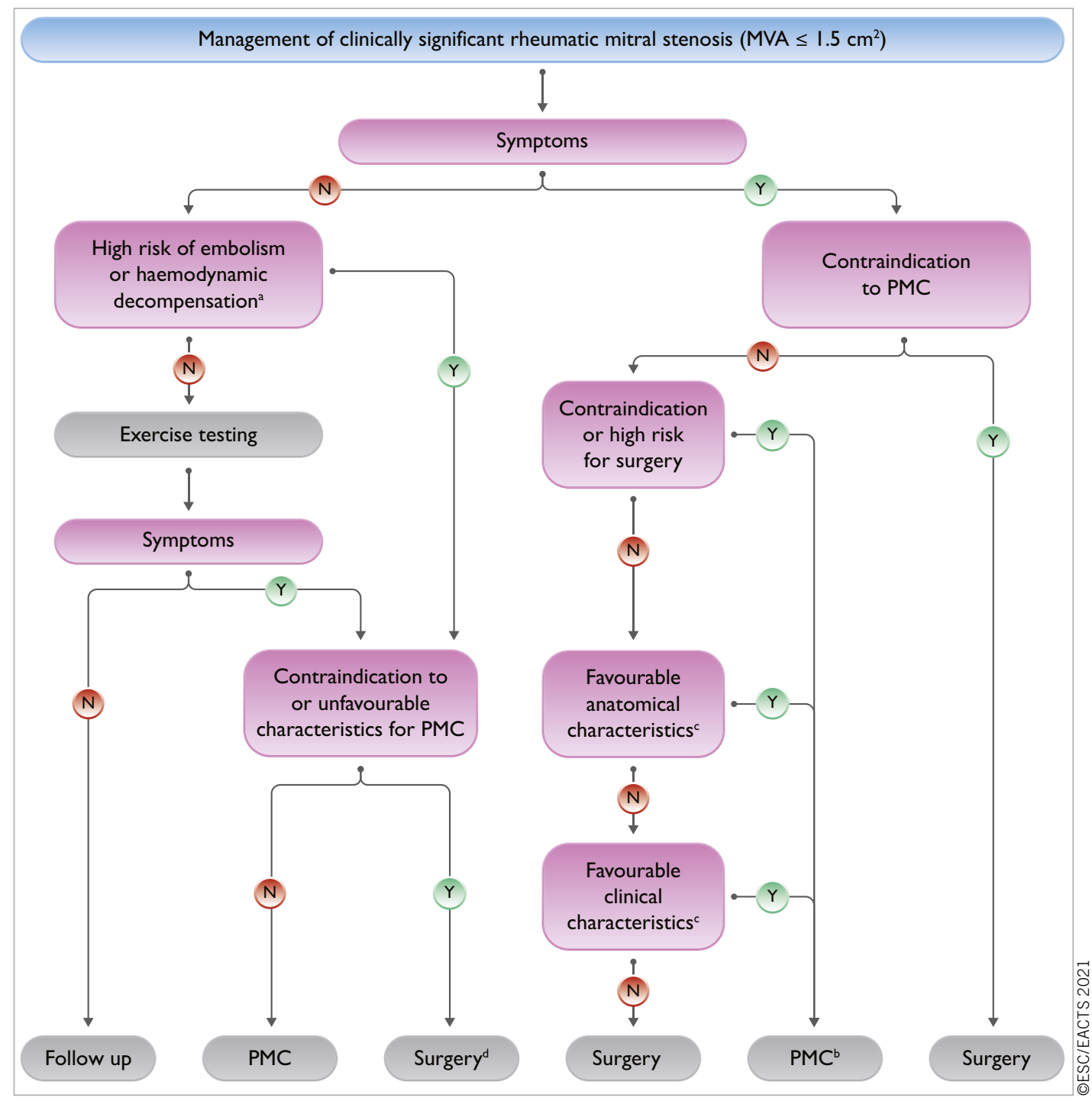

Figure 7. Management of clinically significant rheumatic mitral stenosis (MVA $\left.\leq 1.5 \mathrm{~cm}^{2}\right)$. AF: atrial fibrillation; LA: left atrium/left atrial; MVA: mitral valve area; NCS: non-cardiac surgery; PMC: percutaneous mitral commissurotomy. ${ }^{a}$ High thromboembolic risk: history of systemic embolism, dense spontaneous contrast in the LA, new-onset AF. High-risk of haemodynamic decompensation: systolic pulmonary pressure $>50 \mathrm{mmHg}$ at rest, need for major NCS, desire for pregnancy. ${ }^{b}$ Surgical commissurotomy may be considered by experienced surgical teams in patients with contraindications to PMC. 'See recommendations on indications for PMC and mitral valve surgery in clinically significant mitral stenosis in section 7.2. 'Surgery if symptoms occur for a low level of exercise and operative risk is low.

with less than severe mitral stenosis. Amiodarone is most effective in maintaining the sinus rhythm after cardioversion. In patients in sinus rhythm, OAC is recommended when there has been a history of systemic embolism or a thrombus is present in the LA and should also be considered when TOE shows dense spontaneous echocardiographic contrast or an enlarged LA (M-mode diameter $>50 \mathrm{~mm}$ or LA volume $>60 \mathrm{~mL} / \mathrm{m}^{2}$ ).

\subsubsection{SERIAL TESTING}

Asymptomatic patients with clinically significant mitral stenosis should be followed up yearly by clinical and echocardiographic examinations; and at longer intervals (2-3 years) in case of moderate stenosis. Follow-up of patients after successful PMC is similar to that of asymptomatic patients and should be more frequent if asymptomatic restenosis occurs.

\subsubsection{SPECIAL PATIENT POPULATIONS}

When symptomatic restenosis occurs after surgical commissurotomy or PMC, re-intervention in most cases requires valve replacement, but PMC can be proposed in selected candidates with favourable characteristics if the predominant mechanism is commissural refusion. ${ }^{369}$

In patients with severe rheumatic mitral stenosis combined with severe aortic valve disease, surgery is preferable when it is not contraindicated. The management of patients in whom surgery is contraindicated is difficult and requires a comprehensive and individualized evaluation by the Heart Team. In cases with severe mitral stenosis associated with moderate aortic valve disease, PMC can be performed to postpone the surgical treatment of both valves. In patients with severe tricuspid regurgitation, PMC 
may be considered in selected patients with sinus rhythm, moderate atrial enlargement, and severe functional tricuspid regurgitation secondary to pulmonary hypertension. In other cases, surgery on both valves is preferred. ${ }^{371}$

In the elderly population with rheumatic mitral stenosis when surgery is high risk, PMC is a useful option, even if as palliative care. ${ }^{364,367,368}$ Treatment of patients with low-gradient severe mitral stenosis (MVA $\leq 1.5 \mathrm{~cm}^{2}$, mean gradient $<10 \mathrm{mmHg}$ ) is difficult, as these patients are older and have less optimal anatomy. ${ }^{372}$

\subsection{DEGENERATIVE MITRAL STENOSIS WITH MITRAL ANNULAR CALCIFICATION}

MAC is a distinct entity that differs from rheumatic mitral stenosis. Usually, these patients are elderly and may have significant comorbidities including disease of other valves. Overall, the prognosis is poor due to high-risk profile and technical anatomic challenges resulting from the presence of annular calcification. ${ }^{373}$ Between $9 \%$ and $15 \%$ of the general population may have MAC, with higher frequency in elderly patients (40\%). ${ }^{67,374-376}$ Furthermore, almost half of patients with aortic stenosis undergoing TAVI have MAC, and the disease is severe in $9.5 \%$ of cases. $^{359,377}$ Severe MAC may result in mitral stenosis (more frequently) or mitral regurgitation, or both.

\subsubsection{EVALUATION}

In patients with degenerative mitral stenosis and MAC, the echocardiographic evaluation of the disease severity is difficult and the usual parameters lack validation. Planimetry is less reliable due to diffuse calcium and irregular orifice. The mean transmitral gradient has been shown to have prognostic value. ${ }^{378}$ For the evaluation of severity, it is necessary to take into account the abnormalities of LA and LV compliance before indicating an intervention. If an intervention is planned, echocardiography is used for initial evaluation and CCT is necessary to assess the degree and location of calcification and to evaluate the feasibility of an intervention. ${ }^{379}$

\subsubsection{INDICATIONS FOR INTERVENTION}

Treatment options, including transcatheter and surgical approaches, are high-risk procedures and evidence from randomized trials is lacking. Even if the procedure is done successfully and the transvalvular gradient is reduced, due to low compliance of the LA and LV the mean atrial pressure may remain elevated.

In elderly patients with degenerative mitral stenosis and MAC, surgery is technically challenging and high risk. ${ }^{380}$ As there is no commissural fusion, degenerative mitral stenosis is not amenable to PMC. ${ }^{359}$ In symptomatic inoperable patients with suitable anatomy, preliminary experience showed that transcatheter mitral valve implantation (in mitral position, using an inverted balloon-expandable TAVI prosthesis), is feasible in selected patients with severe mitral stenosis, when performed by experienced operators after careful pre-planning using multimodality imaging. ${ }^{379}$ The largest case series reported to date included only 116 patients. ${ }^{381}$ However, operative mortality is high, in particular due to the risk of LVOT obstruction and mid-term results are less favourable compared to mitral valvein-valve procedures. ${ }^{382,383}$ The most recent case series show that results are improving owing to better patient selection and the use of different accesses, as well as concomitant or preventive measures such as alcohol septal ablation ${ }^{384}$ or laceration/resection of the anterior leaflet. ${ }^{385-387}$

Recently, a preliminary case series suggested that transcatheter mitral valve replacement using a dedicated prosthesis is feasible and can result in symptom improvement. ${ }^{388}$

\section{Tricuspid regurgitation}

Moderate or severe tricuspid regurgitation is observed in $0.55 \%$ of the general population and its prevalence increases with age, affecting about $4 \%$ of the patients aged 75 years or more. ${ }^{389}$ Aetiology is secondary in $\geq 90 \%$ of cases, due to pressure and/or volume overload mediated RV dilatation or enlarged right atrium and tricuspid annulus due to chronic AF. Secondary tricuspid regurgitation is associated with left-sided valvular or myocardial dysfunction in most cases, whereas it is isolated in $8.1 \%$ of subjects and independently related to mortality. ${ }^{389}$ Secondary tricuspid regurgitation may also develop late after left-sided valve surgery. ${ }^{390,391}$

Causes of primary tricuspid regurgitation include infective endocarditis [especially in intravenous (i.v.) drug addicts], rheumatic heart disease, carcinoid syndrome, myxomatous disease, endomyocardial fibrosis, congenital valve dysplasia (e.g. Ebstein's anomaly), thoracic trauma, and iatrogenic valve damage.

Atrial fibrillation induces annular remodelling even in the absence of left-heart disease. ${ }^{392}$ Cardiac implantable electronic device-lead implantation leads to progressive tricuspid regurgitation in $20-30 \%$ of the patients ${ }^{393-395}$ and predicts its progression over time. ${ }^{396}$

In patients with heart failure and reduced LVEF, secondary tricuspid regurgitation is a very frequent finding and is an independent predictor of clinical outcomes. ${ }^{397}$

\subsection{EVALUATION}

Tricuspid regurgitation should be evaluated first by echocardiography. In primary tricuspid regurgitation, specific abnormalities of the valve can be identified. In secondary tricuspid regurgitation, annular dilatation, along with $\mathrm{RV}$ and right atrium dimensions, as well as RV function should be measured, owing to their prognostic relevance. ${ }^{398}$ In experienced laboratories, RV strain ${ }^{27}$ and/or 3D measurements of RV volumes ${ }^{399,400}$ may be considered to overcome the existing limitations of conventional RV function indices. ${ }^{102}$ When available, CMR is the preferred method to assess the $\mathrm{RV}^{400}$ due to its high accuracy and reproducibility. ${ }^{401}$

Echocardiographic evaluation of tricuspid regurgitation severity is based on an integrative approach considering multiple qualitative and quantitative parameters (Table 9). Due to the non-circular and non-planar shape of the regurgitant orifice, biplane vena contracta width should be considered in addition to the conventional 
Table 9. Echocardiographic criteria for grading severity of tricuspid regurgitation.

Qualitative

\begin{tabular}{|l|l|}
\hline Tricuspid valve morphology & Abnormal/flail \\
\hline Colour flow regurgitant jet & $\begin{array}{l}\text { Very large central jet or eccentric } \\
\text { wall impinging jet }^{\mathrm{a}}\end{array}$ \\
\hline CW signal of regurgitant jet & Dense/triangular with early peaking \\
\hline
\end{tabular}

Semiquantitative

\begin{tabular}{|l|l|}
\hline Vena contracta width $(\mathrm{mm})$ & $>7^{\mathrm{a}, \mathrm{b}}$ \\
\hline PISA radius $(\mathrm{mm})$ & $>9^{\mathrm{c}}$ \\
\hline Hepatic vein flow & Systolic flow reversal \\
\hline Tricuspid inflow & E-wave dominant $\geq 1 \mathrm{~m} / \mathrm{s}^{\mathrm{d}}$ \\
\hline Quantitative & $\geq 40$ \\
\hline EROA $\left(\mathrm{mm}^{2}\right)$ & $\geq 45$ \\
\hline Regurgitant volume $(\mathrm{mL} /$ beat $)$ & $\mathrm{RV}, \mathrm{RA}$, inferior vena cava \\
\hline $\begin{array}{l}\text { Enlargement of cardiac } \\
\text { chambers/vessels }\end{array}$ &
\end{tabular}

CW: continuous wave; EROA: effective regurgitant orifice area;

PISA: proximal isovelocity surface area; RA: right atrium/right atrial;

RV: right ventricle/right ventricular; TR: tricuspid regurgitation.

${ }^{a}$ At a Nyquist limit of $50-60 \mathrm{~cm} / \mathrm{s}$. ${ }^{\text {b}}$ Preferably biplane. ${ }^{\text {CB }}$ Baseline

Nyquist limit shift of $28 \mathrm{~cm} / \mathrm{s}$. In the absence of other causes of elevated RA pressure.

2D measurement. ${ }^{402}$ Similarly, underestimation of tricuspid regurgitation severity by the PISA method may occur. ${ }^{403}$ In case of inconsistent findings, the 3D vena contracta area may be evaluated, although diverging cut-offs have been reported. ${ }^{402,404-406}$ Recently, a new grading scheme including two additional grades ('massive' and 'torrential') has been proposed ${ }^{407}$ and used in clinical studies on transcatheter interventions. ${ }^{408,409}$ Studies showed an incremental prognostic value of the two additional grades (massive and torrential) in terms of mortality and rehospitalization for heart failure in patients with advanced disease. ${ }^{410-412}$

Alternatively, calculation of the tricuspid regurgitant volume by CMR using RV volumetry may be helpful.

Importantly, estimation of pulmonary pressures using Doppler gradient may be impossible or might underestimate the severity of pulmonary hypertension in the presence of severe tricuspid regurgitation, justifying cardiac catheterization to evaluate pulmonary vascular resistances. ${ }^{413}$

\subsection{INDICATIONS FOR INTERVENTION}

Severe tricuspid regurgitation is associated with impaired survival $^{389,414-416}$ and worsening heart failure. ${ }^{397,417}$ In clinical practice, tricuspid valve interventions are underused and often initiated too late. ${ }^{418-420}$ Appropriate timing of intervention is crucial to avoid irreversible RV damage and organ failure with subsequent increased surgical risk ${ }^{421,422}$ (see table of recommendations on indications for intervention in tricuspid valve disease in section 9 and Figure 8).

Surgery is recommended in symptomatic patients with severe primary tricuspid regurgitation. In selected asymptomatic or mildly symptomatic patients who are appropriate for surgery, an intervention should also be considered when RV dilatation or declining RV function is observed. However, exact thresholds have not yet been defined.

According to observational data, tricuspid valve repair should be performed liberally during left-sided surgery in patients with secondary tricuspid regurgitation. Indeed, it does not increase operative risk, but promotes reverse remodelling of the RV and improves functional status when annular dilatation is present, even in the absence of severe tricuspid regurgitation. ${ }^{423-427}$

The benefit of surgical correction of isolated secondary tricuspid regurgitation compared to medical treatment is not well established $^{428}$ and the procedure has a non-negligible risk of periprocedural mortality and morbidity when patients present late. ${ }^{429-432}$ However, in carefully selected candidates, surgery can be performed safely with good long-term survival. ${ }^{418,433}$ It should therefore be considered early in selected symptomatic patients appropriate for surgery, as well as in those with no or mild symptoms, RV dilatation and severe tricuspid regurgitation. Although a tricuspid annular pulmonary systolic excursion (TAPSE) $<17 \mathrm{~mm}$ has been associated with worse prognosis in patients with secondary tricuspid regurgitation, ${ }^{398,434}$ thresholds for severe RV dysfunction making intervention futile have not yet been defined.

Reoperation on the tricuspid valve in new-onset or worsening secondary tricuspid regurgitation after left-sided surgery carries a high procedural risk, possibly due to late referral and subsequent poor clinical condition. ${ }^{435}$ To improve prognosis, treatment of severe tricuspid regurgitation in this challenging scenario should be considered even in asymptomatic patients if there are signs of $\mathrm{RV}$ dilatation or decline in RV function (after exclusion of leftsided valve dysfunction, severe RV or LV dysfunction and severe pulmonary vascular disease/hypertension).

Whenever possible, annuloplasty with prosthetic rings is preferable to valve replacement, ${ }^{423,430,436}$ which should only be considered when the tricuspid valve leaflets are tethered and the annulus severely dilated. In presence of a cardiac implantable electronic device lead, the technique used should be adapted to the patient's condition and the surgeon's experience. ${ }^{437}$

TTVI are under clinical development. Early registry and study data demonstrated the feasibility to reduce tricuspid regurgitation using various systems, enabling either leaflet approximation, ${ }^{408,438-440}$ direct annuloplasty, ${ }^{409,441}$ or valve replacement, ${ }^{442-444}$ with subsequent symptomatic and haemodynamic improvement. ${ }^{445,446}$ In a propensity-score-matched study comparing medical treatment to TTVI, all-cause mortality and rehospitalizations at 1 year were lower among the patients who received the interventional treatment. ${ }^{447}$ Several RCTs will investigate the efficacy of TTVI against medical treatment.

Therefore, TTVI may be considered by the Heart Team at experienced Heart Valve Centres in symptomatic, inoperable, anatomically eligible patients in whom symptomatic or prognostic improvement can be expected. For detailed anatomical evaluation, TOE and CCT may be preferred owing to higher spatial resolution. ${ }^{448,449}$ 


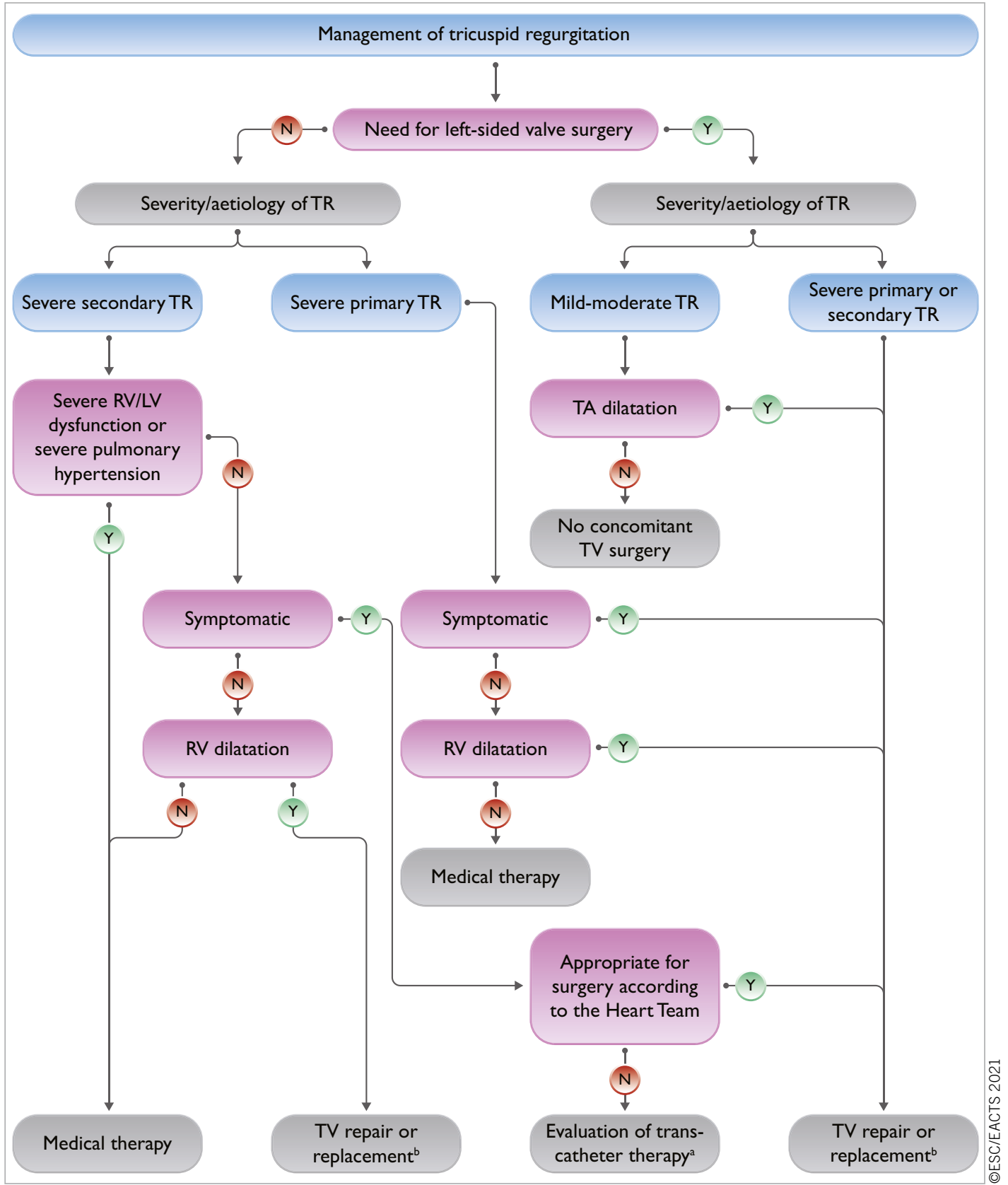

Figure 8. Management of tricuspid regurgitation. LV: left ventricle/left ventricular; $R V$ : right ventricle/right ventricular; TA: tricuspid annulus; TR: tricuspid regurgitation; TV: tricuspid valve. ${ }^{a}$ The Heart Team with expertise in the treatment of tricuspid valve disease evaluates anatomical eligibility for transcatheter therapy including jet location, coaptation gap, leaflet tethering, potential interference with pacing lead. ${ }^{b}$ Replacement when repair is not feasible.

\subsection{MEDICAL THERAPY}

Diuretics are useful in the presence of right heart failure. To counterbalance the activation of the renin-angiotensin-aldosterone system associated with hepatic congestion, the addition of an aldosterone antagonist may be considered. ${ }^{247}$ Dedicated treatment of pulmonary hypertension is indicated in specific cases. Although data are limited, rhythm control may help to decrease tricuspid regurgitation and contain annular dilatation in patients with chronic AF. ${ }^{450}$ Importantly, in the absence of advanced RV dysfunction or severe pulmonary hypertension, none of the abovementioned therapies should delay referral for surgery or transcatheter therapy.

\section{Tricuspid stenosis}

Tricuspid stenosis is often combined with tricuspid regurgitation and most frequently of rheumatic origin. It is therefore usually associated with left-sided valve lesions, particularly mitral stenosis. Other causes are rare, including congenital, carcinoid and 
drug-induced valve diseases, Whipple's disease, endocarditis, and large right atrial tumour.

\subsection{EVALUATION}

Echocardiography provides the most useful information. Tricuspid stenosis is often overlooked and requires careful evaluation. Echocardiographic evaluation of valve anatomy and subvalvular apparatus is important to assess valve reparability. No generally accepted grading of tricuspid stenosis severity exists, but a mean echocardiographic transvalvular gradient $\geq 5 \mathrm{mmHg}$ at normal heart rate is considered indicative of significant tricuspid stenosis. ${ }^{362}$

\subsection{INDICATIONS FOR INTERVENTION}

Intervention on the tricuspid valve is usually performed concomitantly during procedures for left-sided valve disease in patients who are symptomatic despite medical therapy. Although the lack of pliable leaflet tissue is a main limitation for valve repair, the choice between repair and replacement depends on anatomy and surgical expertise. Owing to satisfactory long-term durability, biological prostheses are usually preferred over mechanical valves, which have a high risk of thrombosis. ${ }^{451}$

Percutaneous tricuspid balloon valvuloplasty has been performed in a limited number of cases, either alone or in combination with PMC. It frequently induces significant regurgitation and long-term results are lacking. ${ }^{452}$ It can be considered in rare cases with anatomically suitable valves, when tricuspid stenosis is isolated or additional mitral stenosis can also be treated interventionally (see recommendations on indications for PMC and mitral valve surgery in clinically significant mitral stenosis in section 7).

\subsection{MEDICAL THERAPY}

Diuretics are useful in the presence of heart failure symptoms but are of limited long-term efficacy.

\section{Combined and multiple-valve diseases}

Significant stenosis and regurgitation can be found on the same valve. Disease of multiple valves may be encountered in several conditions, particularly in rheumatic and congenital heart disease, but also less frequently in degenerative valve disease. There is a lack of data on combined or multiple-valve disease. ${ }^{453-460}$ This does not allow for evidence-based recommendations. The general principles for the management of combined or multiple-valve disease are as follows:

- When either stenosis or regurgitation is predominant, management follows the recommendations concerning the predominant VHD. When the severity of both stenosis and regurgitation is balanced, indications for interventions should be based on symptoms and objective consequences rather than on the indices of severity of stenosis or regurgitation. ${ }^{453-456}$ In this setting, Doppler pressure gradient reflects the global haemodynamic burden (stenosis and regurgitation) of the valve lesion. ${ }^{453}$

\section{Recommendations on indications for intervention in tricuspid valve disease}

\begin{tabular}{|c|c|c|}
\hline Recommendations & Class $^{\mathrm{a}}$ & Level $^{\mathrm{b}}$ \\
\hline \multicolumn{3}{|l|}{ Recommendations on tricuspid stenosis } \\
\hline $\begin{array}{l}\text { Surgery is recommended in symptomatic patients } \\
\text { with severe tricuspid stenosis. }^{c}\end{array}$ & I & C \\
\hline $\begin{array}{l}\text { Surgery is recommended in patients with severe } \\
\text { tricuspid stenosis undergoing left-sided valve } \\
\text { intervention. }^{d}\end{array}$ & $\mathbf{I}$ & C \\
\hline \multicolumn{3}{|l|}{ Recommendations on primary tricuspid regurgitation } \\
\hline $\begin{array}{l}\text { Surgery is recommended in patients with severe } \\
\text { primary tricuspid regurgitation undergoing } \\
\text { leftsided valve surgery. }\end{array}$ & I & C \\
\hline $\begin{array}{l}\text { Surgery is recommended in symptomatic patients } \\
\text { with isolated severe primary tricuspid } \\
\text { regurgitation without severe RV dysfunction. }\end{array}$ & I & C \\
\hline $\begin{array}{l}\text { Surgery should be considered in patients with } \\
\text { moderate primary tricuspid regurgitation } \\
\text { undergoing left-sided valve surgery. }\end{array}$ & Ila & C \\
\hline $\begin{array}{l}\text { Surgery should be considered in asymptomatic or } \\
\text { mildly symptomatic patients with isolated severe } \\
\text { primary tricuspid regurgitation and RV dilatation } \\
\text { who are appropriate for surgery. }\end{array}$ & Ila & C \\
\hline \multicolumn{3}{|c|}{ Recommendations on secondary tricuspid regurgitation } \\
\hline $\begin{array}{l}\text { Surgery is recommended in patients with severe } \\
\text { secondary tricuspid regurgitation undergoing } \\
\text { left-sided valve surgery.423-427 }\end{array}$ & I & B \\
\hline $\begin{array}{l}\text { Surgery should be considered in patients with } \\
\text { mild or moderate secondary tricuspid } \\
\text { regurgitation with a dilated annulus ( } \geq 40 \mathrm{~mm} \text { or } \\
>21 \mathrm{~mm} / \mathrm{m}^{2} \text { by } 2 \mathrm{D} \text { echocardiography) undergoing } \\
\text { left-sided valve surgery. }\end{array}$ & Ila & B \\
\hline $\begin{array}{l}\text { Surgery should be considered in patients with } \\
\text { severe secondary tricuspid regurgitation (with or } \\
\text { without previous left-sided surgery) who are } \\
\text { symptomatic or have RV dilatation, in the } \\
\text { absence of severe RV or LV dysfunction and } \\
\text { severe pulmonary vascular disease/ } \\
\text { hypertension. }{ }^{418,433 \text { e }}\end{array}$ & Ila & B \\
\hline $\begin{array}{l}\text { Transcatheter treatment of symptomatic } \\
\text { secondary severe tricuspid regurgitation may be } \\
\text { considered in inoperable patients at a Heart Valve } \\
\text { Centre with expertise in the treatment of } \\
\text { tricuspid valve disease. }{ }^{f}\end{array}$ & Ilb & C \\
\hline
\end{tabular}

2D: two-dimensional; LV: left ventricle/left ventricular; PMC: percutaneous mitral commissurotomy; RV: right ventricle/right ventricular. ${ }^{\mathrm{a}}$ Class of recommendation. ${ }^{\mathrm{b}}$ Level of evidence.

cPercutaneous balloon valvuloplasty can be attempted as a first approach if tricuspid stenosis is isolated. dPercutaneous balloon valvuloplasty can be attempted if PMC can be performed on the mitral valve. ${ }^{e}$ In patients with previous surgery recurrent left-sided valve dysfunction needs to be excluded. ${ }^{\mathrm{f}}$ Transcatheter treatment can be performed according to Heart Team at experienced valve centres in anatomically eligible patients in whom improvement of quality of life or survival can be expected.

- Besides the separate assessment of each valve lesion, it is necessary to consider the interaction between the different valve lesions. As an illustration, associated mitral regurgitation may lead to underestimation of the severity of aortic stenosis, as decreased stroke volume due to mitral regurgitation lowers the flow across the aortic valve and hence the aortic gradient. ${ }^{453}$ This underlines the need to combine different measurements, including assessment of valve areas, if possible using methods that are less dependent on loading conditions, such as planimetry. ${ }^{457}$ 
- Indications for intervention are based on global assessment of the consequences of the different valve lesions (i.e. symptoms or presence of LV dilatation or dysfunction). Intervention can be considered for non-severe multiple lesions associated with symptoms or leading to LV impairment. ${ }^{453}$

- The decision to intervene on multiple valves should take into account the age, comorbidities, and risk of combined procedures, and should be made by the Heart Team after precise and comprehensive evaluation of valve lesions and their interactions with each other. ${ }^{453,461}$ The risk of combined intervention should be weighed against the evolution of untreated valve disease and the inherent risk of subsequent intervention.

- The choice of surgical technique/interventional procedure should take into account the presence of the other VHD. ${ }^{453,458,459,461}$

- When interventional procedures are considered, staged procedures may be preferable in cases with aortic stenosis and mitral regurgitation (see section 5.5). Improved 1-year survival after combined transcatheter treatment of mitral and tricuspid regurgitation has been reported compared to mitral regurgitation alone. ${ }^{263}$ PMC may delay surgery, in situations such as severe mitral stenosis associated with moderate aortic regurgitation.

The management of specific associations of VHD is detailed in the individual sections of this document.

\section{Prosthetic valves}

\subsection{CHOICE OF PROSTHETIC VALVE}

Factors for valve selection are the patient's life expectancy, lifestyle, and environmental factors, bleeding and thromboembolic risks related to anticoagulation, potential for surgical or transcatheter re-intervention, and, importantly, informed patient preference. Generally, biological heart valves (BHVs) should be

\begin{tabular}{|c|c|c|}
\hline \multicolumn{3}{|l|}{ Recommendations for prosthetic valve selection } \\
\hline Recommendations & Class $^{\mathrm{a}}$ & Level $^{b}$ \\
\hline \multicolumn{3}{|l|}{ Mechanical prostheses } \\
\hline $\begin{array}{l}\text { A mechanical prosthesis is recommended } \\
\text { according to the desire of the informed patient } \\
\text { and if there are no contraindications to longterm } \\
\text { anticoagulation.c }\end{array}$ & I & C \\
\hline $\begin{array}{l}\text { A mechanical prosthesis is recommended in } \\
\text { patients at risk of accelerated SVD. }\end{array}$ & I & C \\
\hline $\begin{array}{l}\text { A mechanical prosthesis should be considered in } \\
\text { patients already on anticoagulation because of } \\
\text { a mechanical prosthesis in another valve } \\
\text { position. }\end{array}$ & Ila & C \\
\hline $\begin{array}{l}\text { A mechanical prosthesis should be considered in } \\
\text { patients aged }<60 \text { years for prostheses in the } \\
\text { aortic position and aged }<65 \text { years for } \\
\text { prostheses in the mitral position. }{ }^{462,464 \mathrm{e}}\end{array}$ & Ila & B \\
\hline $\begin{array}{l}\text { A mechanical prosthesis should be considered in } \\
\text { patients with a reasonable life expectancy for } \\
\text { whom future redo valve surgery or TAVI (if } \\
\text { appropriate) would be at high risk. }{ }^{f}\end{array}$ & Ila & C \\
\hline $\begin{array}{l}\text { A mechanical prosthesis may be considered in } \\
\text { patients already on long-term anticoagulation } \\
\text { due to the high risk for thromboembolism. }{ }^{f}\end{array}$ & Illb & C \\
\hline
\end{tabular}

\section{Biological prostheses}

A bioprosthesis is recommended according to the desire of the informed patient.

A bioprosthesis is recommended when goodquality anticoagulation is unlikely (adherence problems, not readily available), contraindicated because of high bleeding risk (previous major bleed, comorbidities, unwillingness, adherence problems, lifestyle, occupation) and in those patients whose life expectancy is lower than the presumed durability of the bioprosthesis.

A bioprosthesis is recommended in case of reoperation for mechanical valve thrombosis despite good long-term anticoagulant control.

A bioprosthesis should be considered in patients for whom there is a low likelihood and/or a low operative risk of future redo valve surgery.

A bioprosthesis should be considered in young women contemplating pregnancy.

A bioprosthesis should be considered in patients aged $>65$ years for a prosthesis in the aortic position or aged $>70$ years in a mitral position.

A bioprosthesis may be considered in patients already on long-term NOACs due to the high risk for thromboembolism. ${ }^{466-469} \mathrm{f}$

\begin{tabular}{|c|c|c|}
\hline & I & C \\
I & C \\
\hline ity & & \\
\hline I & C \\
\hline Ila & C \\
\hline Ila & C \\
\hline Ila & C \\
\hline
\end{tabular}

AF: atrial fibrillation; NOAC: non-vitamin $\mathrm{K}$ antagonist oral anticoagulant; SVD: structural valve deterioration; TAVI: transcatheter aortic valve implantation. ${ }^{\mathrm{a}} \mathrm{Class}$ of recommendation. ${ }^{\mathrm{b}}$ Level of evidence. 'Increased bleeding risk because of comorbidities, adherence concerns or geographic, lifestyle or occupational conditions. dYoung age ( $<40$ years), hyperparathyroidism, haemodialysis. ${ }^{e}$ In patients $60-65$ years of age who should receive an aortic prosthesis and those between 65 and 70 years of age in the case of mitral prosthesis, both valves are acceptable and the choice requires careful analysis of factors other than age. ${ }^{\mathrm{f}}$ Risk factors for thromboembolism are AF, previous unprovoked proximal deep venous thromboembolism and/or symptomatic pulmonary embolism, hypercoagulable state, antiphospholipid antibody. ${ }^{\mathrm{B}}$ Life expectancy should be estimated at $>10$ years according to age, sex, comorbidities, and country-specific life expectancy.

preferred in patients with shorter anticipated survival or comorbidities that may lead to further surgical procedures, and those who are at increased risk for bleeding complications. Thromboembolic complications are less frequent in pregnant women with BHVs.

In a nationwide observational study, patients aged 45 to 54 with surgical aortic BHV implantation and those aged 40 to 70 years with surgical mitral BHV implantation had a significantly higher 15 -year mortality than those with a mechanical heart valve (MHV). An analysis of patients 55 to 64 years of age showed no difference in mortality between aortic BHV and MHV prosthesis. ${ }^{462}$ However, an earlier systematic review ${ }^{463}$ and a recent meta-analysis ${ }^{464}$ of studies comparing aortic MHVs and BHVs showed a significant mortality reduction with MHVs in patients $\leq 60$ and in those 50-70 years of age, respectively. All these studies are limited by their predominantly observational nature and missing information on the type of prostheses implanted. There is no new high-quality evidence supporting a decrease in the established age cut-off for prosthesis selection.

The best aortic valve substitute for younger adults remains unclear. In appropriately selected patients, replacement of the aortic valve using an autograft may be performed, with long-term 
survival rates and valve-related reoperation that are comparable to those achieved with a MHV, but high expertise in aortic root surgery is required. ${ }^{465}$ Strategies for patients with small aortic annulus include root enlargement and use of stentless valves. Although the use of sutureless and rapid-deployment aortic valves may reduce invasiveness, cross-clamp and cardiopulmonary bypass times, and potentially lower perioperative complications of SAVR, there is a lack of a large-scale randomized comparison on both short- and long-term safety, efficacy, and haemodynamic performance of this approach against conventional aortic valve replacement, which remains the gold standard of procedure.

\subsection{BASELINE ASSESSMENT AND FOLLOW-UP}

All patients with prosthetic valves require lifelong follow-up to detect early deterioration in prosthetic function or ventricular function, or progressive disease of another heart valve. ${ }^{314}$ Clinical assessment should be performed yearly or as soon as possible if new cardiac symptoms occur. TTE should be performed if any new symptoms occur or if complications are suspected. After transcatheter, as well as surgical implantation of a BHV, echocardiography, including measurement of transprosthetic gradients, should be performed within 30 days after valve implantation (i.e. baseline), at 1 year, and annually thereafter. ${ }^{470}$ TOE should be considered if TTE is of poor quality and in all cases of suspected prosthetic dysfunction (especially if the prosthesis is in the mitral position) or endocarditis. ${ }^{314,471}$ Cinefluoroscopy for MHVs and CCT scanning provide useful additional information if valve thrombus or pannus are suspected to impair valve function. ${ }^{314}$

\subsection{ANTITHROMBOTIC MANAGEMENT}

\subsubsection{MECHANICAL PROSTHESES}

\subsubsection{Postoperative anticoagulation management}

MHVs require lifelong treatment with VKA guided by the INR $^{472,473}$ NOACs currently have no role in patients with MHVs. ${ }^{474}$ Treatment with VKA should be started on the first postoperative day in combination with bridging therapy [with therapeutic doses of either unfractionated heparin (UFH) or off-label use of low-molecular-weight heparin (LMWH)] until therapeutic INR is achieved. ${ }^{475}$ Similar safety and efficacy outcomes have been reported following bridging with either UFH or LMWH. ${ }^{476}$ Once a stable therapeutic INR is reached for $\geq 24 \mathrm{~h}$, bridging can be discontinued. The postoperative risk of thromboembolism peaks about 1 month after implantation, but risks are substantially increased up to 6 months. ${ }^{477,478}$ Long-term prevention of valve thrombosis and thromboembolism after MHV implantation involves effective antithrombotic medication and risk factor modification for thromboembolism. ${ }^{479}$

\subsubsection{Target international normalized ratio}

Target INR should be based upon prosthesis thrombogenicity and patient-related risk factors (Table 10). ${ }^{479}$ It is recommended to target a median INR value rather than a range to avoid considering extreme values in the target range as a valid target INR. High INR variability is a strong independent predictor of adverse events after
Table 10. Target international normalized ratio for mechanical prostheses.

\begin{tabular}{|c|c|c|}
\hline \multirow{2}{*}{$\begin{array}{l}\text { Prosthesis } \\
\text { thrombogenicity }\end{array}$} & \multicolumn{2}{|c|}{ Patient-related risk factors ${ }^{a}$} \\
\hline & None & $\geq 1$ risk factor \\
\hline Low $^{b}$ & 2.5 & 3.0 \\
\hline Medium $^{c}$ & 3.0 & 3.5 \\
\hline High $^{d}$ & 3.5 & 4.0 \\
\hline \multicolumn{3}{|c|}{ 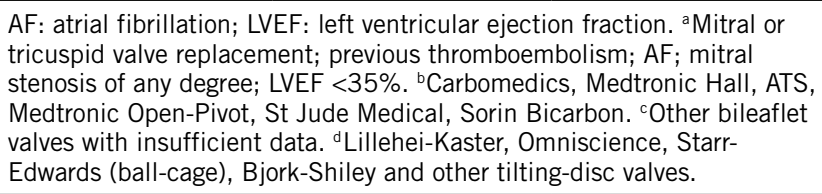 } \\
\hline
\end{tabular}

valve replacement. Although some studies have supported lowering a target INR for aortic MHVs, ${ }^{480,481}$ further evaluation in larger cohorts is warranted before updating current recommendations. The use of self-monitoring INR is associated with a lower rate of VKA-related complications in all ages. ${ }^{482}$ In a trial of lower intensity warfarin plus aspirin (INR 1.5-2.0) or standard warfarin plus aspirin (INR 2.0-3.0) after implantation of the On-X MHV in the aortic position, the similar safety of the two approaches was partly attributed to use of home INR monitoring and high degree of adherence among patients. ${ }^{481}$ Patient's education plays an important role for achieving stable anticoagulation in the therapeutic range. Effective management of patients with unstable INR requires frequent in-clinic testing and dose titration. Because of the lack of good-quality evidence, pharmacogenetic testing cannot be recommended to guide the dosing of VKAs.

\subsubsection{Management of vitamin K antagonist (VKA) overdose} and bleeding

Bleeding increases exponentially with INR $>4.5 .{ }^{483}$ In case of major and/or life-threatening bleeding and in patients who need to undergo urgent surgery, the VKA should be discontinued and $10 \mathrm{mg}$ vitamin $\mathrm{K}$ should be administrated by slow i.v. infusion and repeated every $12 \mathrm{~h}$ if needed. Until the anticoagulation effect is reversed, administration of prothrombin complex concentration (PCC) and/or fresh frozen plasma (FFP) therapy should be initiated according to body weight and pre-treatment INR. The efficacy should be monitored by re-check of INR at $30 \mathrm{~min}$ and every 4-6 $\mathrm{h}$ until normalization. The optimal time to restart anticoagulation should be discussed in relation to location of the bleeding event and interventions performed to stop bleeding and/or to treat an underlying cause. ${ }^{484}$

In the absence of bleeding, the use of PCC and/or FFP therapy is not recommended and the decision to start vitamin $\mathrm{K}$ should be individualized. In asymptomatic patients with INR $>10$, the VKA must be stopped and oral vitamin $\mathrm{K}(2.5-5 \mathrm{mg})$ prescribed, while the INR must be monitored on a daily base for 2 weeks. Multiple RCTs in patients with INR between 4.5 and 10 suggest no difference in bleeding events with vitamin K vs. placebo. ${ }^{483,485}$ Therefore, in such patients, warfarin should be stopped temporarily, and a small dose of oral vitamin K (1-2 mg) can be considered on an individual basis balancing between the risks. Finally, 
asymptomatic patients with INR $<4.5$ require careful downtitration and/or skipping one or more doses. In all patients with MHVs, VKAs must be resumed once the INR achieves the therapeutic range or is slightly elevated.

\subsubsection{Combination of oral anticoagulation (OAC) with antiplatelet drugs}

The addition of low-dose (75-100 mg) acetylsalicylic acid (ASA) to VKA may reduce the incidence of thromboembolism at the cost of bleeding. ${ }^{477}$ Therefore, addition of antiplatelets to VKAs should be reserved for patients at very high risk of thromboembolism where advantages clearly outweigh the risks. ${ }^{486,487}$ In patients with thromboembolism despite adequate INR, low dose (75-100 mg) ASA should be added to VKAs. Management of oral antithrombotic therapy in patients with CAD is summarized in

\section{Supplementary Figure 2.}

\subsubsection{Interruption of anticoagulant therapy for planned} invasive procedures

In patients with MHVs, preoperative bridging with UFH or LMWH before surgery imposes a risk of perioperative bleeding while interrupting anticoagulation results in an increased risk of thromboembolism. ${ }^{488}$ Therefore, anticoagulation in patients with MHVs undergoing elective NCS requires careful management by multidisciplinary consensus. ${ }^{478,489}$ For minor surgical procedures (e.g. dental, cataract, skin incision) in which blood loss is usually small and easily controlled, it is recommended that $\mathrm{OAC}$ is not interrupted. Major surgeries require temporary interruption and therapeutic bridging with either UFH or LMWH, aiming for an INR $<1.5$ (Supplementary Figure 3). Fondaparinux should not be routinely used for bridging, but may have a role in patients with history of heparin-induced thrombocytopenia. ${ }^{490}$

\subsubsection{BIOPROSTHESES}

\subsubsection{Patients with no baseline indication to oral} anticoagulation (OAC)

Surgical bioprostheses: The optimal antithrombotic strategy early after surgical implantation of an aortic BHV remains controversial due to lack of high-quality evidence. Multiple observational studies support the use of VKAs to reduce the risk of thromboembolism. ${ }^{491-493}$ A small randomized trial found that VKA for 3 months significantly increased major bleeding compared with ASA, without reducing the rate of deaths or thromboembolic events, but the statistical power was low for demonstrating a thrombotic benefit. ${ }^{494}$ VKA for 3 months should be considered in all patients with a mitral or tricuspid BHV and ASA or VKA should be considered for 3 months after surgical implantation of an aortic bioprosthesis.

Transcatheter bioprostheses: A meta-analysis of three small RCTs showed a significant increase in major or life-threatening bleeding with dual antiplatelet therapy (DAPT) over ASA at 30 days, with no difference in ischaemic outcomes. ${ }^{495}$ Consistently, the more recent POPular TAVI trial (cohort A) found reduced bleeding and the composite of bleeding or thromboembolic events with ASA compared with DAPT. ${ }^{496}$ A randomized trial was halted prematurely due to safety concerns with a rivaroxaban-based regimen as compared with DAPT, including a higher risk of death or thromboembolic complications and a higher risk of bleeding. ${ }^{497}$ There is a lack of data on the management of antithrombotic therapy after implantation of transcatheter mitral BHVs (e.g. valve-invalve or valve-in-ring) for which 3 months of VKA is commonly prescribed. ${ }^{498}$

\subsubsection{Patients with baseline indication to oral} anticoagulation (OAC)

Surgical bioprostheses: OAC is recommended lifelong for patients with surgical BHVs who have other indications for anticoagulation. The evidence supporting the use of NOACs in preference to VKA has increased since the publication of the 2017 VHD Guidelines. In the RIVER trial, including patients with $\mathrm{AF}$ and a $\mathrm{BHV}$ in the mitral position, the NOAC rivaroxaban was non-inferior to warfarin with respect to a net benefit endpoint at 12 months. ${ }^{499}$ The benefit of NOAC was consistent among subgroups. However, only $20 \%$ of patients were enrolled in the trial before the third postoperative month, which raises a note of caution and calls for additional data in this particular subgroup. In the small ENAVLE trial $(\mathrm{N}=220)$, including patients with and without $\mathrm{AF}$, edoxaban was non-inferior to warfarin for preventing thromboembolism and the

\section{Recommendations for management of antithrombotic therapy after prosthetic valve implantation or valve repair in the perioperative and postoperative periods}

Recommendations

Management of antithrombotic therapy in the perioperative period

It is recommended that VKAs are timely discontinued prior to elective surgery to aim for an INR $<1.5$. $^{\circ}$

Bridging of OAC, when interruption is needed, is recommended in patients with any of the following indications:

- Mechanical prosthetic heart valve.

- AF with significant mitral stenosis.

- AF with a $\mathrm{CHA}_{2} \mathrm{DS}_{2}$-VASc score $\geq 3$ for women or 2 for men. ${ }^{d}$

- Acute thrombotic event within the previous 4 weeks.

- High acute thromboembolic risk. ${ }^{{ }}$

Therapeutic doses of either UFH or subcutaneous LMWH are recommended for bridging. ${ }^{476,504}$

In patients with MHVs, it is recommended to (re)-initiate the VKA on the first postoperative day.

In patients who have undergone valve surgery with an indication for postoperative therapeutic bridging, it is recommended to start either UFH or LMWH 12-24 $\mathrm{h}$ after surgery.

In patients undergoing surgery, it is recommended that aspirin therapy, if indicated, is maintained during the periprocedural period.

In patients treated with DAPT after recent $\mathrm{PCl}$ (within 1 month) who need to undergo heart valve surgery in the absence of an indication for $\mathrm{OAC}$, it is recommended to resume the $\mathrm{P}_{2} \mathrm{Y}_{12}$ inhibitor postoperatively, as soon as there is no concern over bleeding. 
In patients treated with DAPT after recent $\mathrm{PCl}$ (within 1 month) who need to undergo heart valve surgery in the absence of an indication for $\mathrm{OAC}$, bridging $\mathrm{P}_{2} \mathrm{Y}_{12}$ inhibitors with short-acting glycoprotein IIb/IIla inhibitors or cangrelor may be considered.

Patients with an indication to concomitant antiplatelet therapy

After uncomplicated $\mathrm{PCl}$ or ACS in patients requiring long-term $\mathrm{OAC}$, early cessation

( $\leq 1$ week) of aspirin and continuation of dual therapy with $\mathrm{OAC}$ and a P2Y inhibitor (preferably clopidogrel) for up to 6 months (or up to 12 months in ACS) is recommended if the risk of stent thrombosis is low or if concerns about bleeding risk prevail over concerns about risk of stent thrombosis, irrespective of the type of stent used. ${ }^{505-509}$

Discontinuation of antiplatelet treatment in patients treated with an OAC is recommended after 12 months. ${ }^{74,510-512}$

After uncomplicated $\mathrm{PCI}$ or ACS in patients requiring both $O A C$ and antiplatelet therapy, triple therapy with aspirin, clopidogrel and OAC for longer than 1 week should be considered when the risk of stent thrombosis outweighs the risk of bleeding, with the total duration ( $\leq 1$ month) decided according to assessment of these risks and clearly specified at hospital discharge.

In patients treated with a VKA (e.g. MHVs), clopidogrel alone should be considered in selected patients (e.g. HAS-BLED $\geq 3$ or ARC-HBR met and low risk of stent thrombosis) for up to 12 months. ${ }^{512,513}$

In patients requiring aspirin and/or clopidogrel in addition to VKA, the dose intensity of VKA should be considered and carefully regulated with a target INR in the lower part of the recommended target range and a time in the therapeutic range $>65-70 \% .{ }^{505,514}$

Surgical valve replacement

OAC using a VKA is recommended lifelong for all patients with an MHV prosthesis. ${ }^{472,473}$

For patients with a VKA, INR self-management is recommended provided appropriate training and quality control are performed. ${ }^{482}$

$\mathrm{OAC}$ is recommended for patients undergoing implantation of a surgical BHV who have other indications for anticoagulation. ${ }^{\dagger}$

NOACs should be considered over VKA after 3 months following surgical implantation of a BHV in patients with $\mathrm{AF}^{74,499,500,515-518}$

In patients with no baseline indications for OAC, low-dose aspirin (75-100 mg/day) or OAC using a VKA should be considered for the first

3 months after surgical implantation of an aortic BHV. ${ }^{491,494}$

In patients with no baseline indications for OAC, OAC using a VKA should be considered for the first 3 months after surgical implantation of a bioprosthesis in the mitral or tricuspid position. ${ }^{519,520}$

The addition of low-dose aspirin

(75-100 mg/day) to VKA may be considered in selected patients with MHVs in case of concomitant atherosclerotic disease and low risk of bleeding.
The addition of low-dose aspirin

(75-100 mg/day) to VKA should be considered after thromboembolism despite an adequate INR.

NOACs may be considered over VKA within 3 months following surgical implantation of a BHV in mitral position in patients with $\mathrm{AF}^{4}{ }^{499}$

NOACs are not recommended in patients with a mechanical valve prosthesis. ${ }^{474}$

\section{Surgical valve repair}

OAC with VKA should be considered during the first 3 months after mitral and tricuspid repair.

SAPT with low-dose ASA (75-100 mg/day) should be considered for the first 3 months after valve-sparing aortic surgery when there are no other baseline indications to OAC.

\section{Transcatheter aortic valve implantation}

OAC is recommended lifelong for TAVI patients who have other indications for OAC. $501 \mathrm{f}$

Lifelong SAPT is recommended after TAVI in patients with no baseline indication for OAC. ${ }^{495,496,521}$

Routine use OAC is not recommended after TAVI in patients with no baseline indication for OAC. ${ }^{497}$

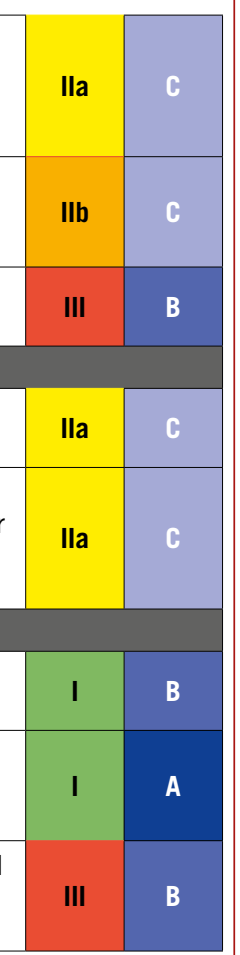

ACS: acute coronary syndrome; AF: atrial fibrillation; ARC-HBR: Academic Research Consortium - high bleeding risk; ASA: acetylsalicylic acid; BHV: biological heart valve; DAPT: dual antiplatelet therapy; INR: international normalized ratio; LMWH: low-molecular-weight heparin; LV: left ventricle/left ventricular; $\mathrm{PCl}$ : percutaneous coronary intervention; MHV: mechanical heart valve; NOAC: non-vitamin K antagonist oral anticoagulant; OAC: oral anticoagulation; SAPT: single antiplatelet therapy; TAVI: transcatheter aortic valve implantation; UFH: unfractionated heparin; VKA: vitamin $\mathrm{K}$ antagonist. ${ }^{\mathrm{a}}$ Class of recommendation. ${ }^{b}$ Level of evidence. ${ }^{c} \leq 5$ days for warfarin and $\leq 3$ days for acenocoumarol. ${ }^{\mathrm{d}} \mathrm{CHA}_{2} \mathrm{DS}_{2}$-VASc, congestive heart failure,

hypertension, age $\geq 75$ ( 2 points), diabetes, prior stroke ( 2 points) - vascular disease, age 65-74, sex category (female). eLV apex thrombus, antithrombin 3 deficit and proteins $\mathrm{C}$ and/or $\mathrm{S}$ deficit. ${ }^{\mathrm{f}} \mathrm{AF}$, venous thromboembolism, hypercoagulable state or, with a lesser degree of evidence, severely impaired LV dysfunction (ejection fraction <35\%).

occurrence of major bleeding in the first 3 months after aortic or mitral surgical bioprosthetic valve implantation or repair, which warrants confirmation in larger investigations. ${ }^{500}$

Transcatheter bioprostheses: In the POPular TAVI trial (cohort B), the incidence of bleeding over a period of 1 month or 1 year was lower with OAC than with OAC plus clopidogrel. ${ }^{501} \mathrm{OAC}$ alone was non-inferior to OAC plus clopidogrel with respect to ischaemic events, but the non-inferiority margin was large. An observational study suggested that there is a higher risk of ischaemic events at 1 year with NOACs compared with VKAs, after adjustment for potential confounders. ${ }^{502}$ Randomized trials comparing NOACs vs. VKAs are ongoing (NCT02943785, NCT02664649). Data on the management of antithrombotic therapy after transcatheter mitral or tricuspid valve implantation are scant. ${ }^{498}$

\subsubsection{VALVE REPAIR}

Observational data suggest comparable risk of thromboembolism with ASA or VKAs following mitral valve repair, ${ }^{503}$ but randomized data are lacking. The high incidence of new-onset AF and its recurrence, the thrombogenic tendency of the non-endothelialized repair components, and a relatively high rate of patients who are resistant 
to ASA establish VKAs as a preferable option for the initial period (e.g. 3 months). However, the potential for bleeding complications in the postoperative phase dictates careful patient selection.

The management of antithrombotic treatment after prosthetic valve implantation or valve repair is summarized in the table of recommendations for management of antithrombotic therapy after prosthetic valve implantation or valve repair and in Figure 9.

\subsection{MANAGEMENT OF PROSTHETIC VALVE DYSFUNCTION AND COMPLICATIONS}

\subsubsection{STRUCTURAL VALVE DETERIORATION}

Definitions of SVD and bioprosthetic valve failure (BVF) were standardized by recent consensus. ${ }^{470,522}$ The comparative durability of TAVI and SAVR BHVs must be ascertained at longer term. Reversible causes of BVF (e.g. endocarditis, thrombosis) should be excluded, and considerations on timing of dysfunction (e.g. for BHV obstruction, mismatch in early phases, thrombosis in later phases) and location of malfunction (e.g. endocarditis or SVD in case of central regurgitation, endocarditis or anatomical/technical factors in case of paravalvular regurgitation) may reveal the most plausible underlying cause and guide clinical decision making.

Percutaneous balloon interventions should be avoided in the treatment of stenotic left-sided bioprostheses. Transcatheter valvein-valve implantation is an option for treating degenerated BHVs in patients with increased surgical risk. ${ }^{227,523-525}$ Redo-TAVI is a safe and feasible option in selected patients, but the risk of PPM in small valves and that of coronary occlusion, as well the possibility for future access to the coronary arteries need to be considered. ${ }^{229,526-528}$ Experience is mostly in aortic BHVs and remains limited for BHVs in the mitral position and even more so in the tricuspid position ${ }^{529-532}$ for which valve-in-valve procedures may be reasonable in patients at increased surgical risk. ${ }^{531,533}$ Valve-inring mitral procedures are also acceptable in selected candidates, while the role of valve-in-ring tricuspid procedures remains uncertain. It is necessary for the Heart Team to discuss every patient and choose the best individualized approach. Careful pre-procedural planning is needed to minimize the risk of coronary artery obstruction and enable future coronary re-access in aortic BHV



Figure 9. Antithrombotic therapy for valve prostheses. AF: atrial fibrillation; ASA: acetylsalicylic acid; CAD: coronary artery disease; DAPT: dual antiplatelet therapy; INR: international normalized ratio; LMWH: low-molecular-weight heparin; LV: left ventricle/left ventricular; $M H V$ : mechanical heart valve; MVR: mitral valve replacement or repair; OAC: oral anticoagulation; SAPT: single antiplatelet therapy; SAVR: surgical aortic valve replacement; TAVI: transcatheter aortic valve implantation; TVR: tricuspid valve replacement or repair; UFH: unfractionated heparin; VKA: vitamin K antagonist. Colour coding corresponds to class of recommendation. 
re-interventions if necessary. For mitral re-interventions the risk of LVOT obstruction should be carefully evaluated. ${ }^{534}$

\subsubsection{NON-STRUCTURAL VALVE DYSFUNCTION}

\subsubsection{Patient-prosthesis mismatch}

Patient-prosthesis mismatch (PPM) significantly decreases longterm survival, correlates with SVD and increases readmission rates for both heart failure and reoperation. ${ }^{535-537}$ Efforts to prevent PPM should receive more emphasis to improve long-term survival after either SAVR or TAVI. ${ }^{538}$

\subsubsection{Paravalvular leak and haemolysis}

Blood tests for haemolysis should be part of routine follow-up after valve replacement. Diagnosis of haemolytic anaemia requires TOE to detect paravalvular leaks for prostheses in the mitral position if TTE is not contributory. Reoperation is recommended if the paravalvular leak is related to endocarditis or causes haemolysis requiring repeated blood transfusions or leading to severe symptoms. Transcatheter closure of a paravalvular leak is feasible, but experience is limited and there is presently no conclusive evidence to show consistent efficacy. ${ }^{539}$ Transcatheter closure of paravalvular leaks should be considered for anatomically suitable paravalvular leaks in candidates selected by the Heart Team. ${ }^{540}$ Medical therapy (including iron supplementation, beta-blockers, and erythropoietin) is indicated in patients with severe haemolytic anaemia when contraindications to surgical or transcatheter closure are present. ${ }^{540}$

\subsubsection{ENDOCARDITIS}

The management of patients with endocarditis should follow the relevant guidelines. ${ }^{4}$

\subsubsection{THROMBOSIS}

\subsubsection{General comments}

Obstructive valve thrombosis should be suspected promptly in any patient with any type of prosthetic valve who presents with recent dyspnoea or an embolic event. The diagnosis should be confirmed by TTE and TOE, cinefluoroscopy, or CCT if promptly available. ${ }^{268,314}$ Valve thrombosis occurs mainly in MHVs. However, cases of thrombosis of BHVs have also been reported after surgery or transcatheter valve implantation. ${ }^{541}$ Thrombus on BHVs can present as hypo-attenuated leaflet thickening (HALT) with relatively normal leaflet motion, HALT with reduced leaflet motion but normal gradients, and clinical valve thrombosis with elevated gradients. Distinguishing between thrombus and pannus by means of CCT is important to guide decision making.

\subsubsection{Valve thrombosis}

The management of MHVs thrombosis is high risk, whatever the option taken. Fibrinolysis carries risks of bleeding, systemic embolism, and recurrent thrombosis. ${ }^{542}$ Emergency valve replacement is recommended for obstructive prosthetic valve thrombosis in critically ill patients without a contraindication to surgery. Management of non-obstructive thrombosis of an MHV depends mainly on the occurrence of a thromboembolic event and the size of the thrombus. Surgery should be considered for a large $(>10 \mathrm{~mm}$ ) non-obstructive prosthetic valve thrombus that is complicated by embolism or persists despite optimal anticoagulation. ${ }^{543}$
Fibrinolysis may be considered if surgery is not an option or is very high risk for the treatment of thrombosis of right-sided prostheses, but carries a risk of bleeding and thromboembolism. Anticoagulation using a VKA and/or UFH is the first-line treatment of BHV thrombosis. Because BHV thrombosis is associated with recurrence and early prosthetic degeneration, indefinite anticoagulation should be considered after a confirmed episode, but this strategy must be balanced against an increased risk of bleeding $^{544,545}$ (Figure 10).

\subsubsection{Subclinical leaflet thrombosis}

HALT is detected by CCT in $12.4 \%$ and $32.4 \%$ of TAVI patients on OAC or DAPT at 3 months, respectively. ${ }^{546}$ The clinical significance of these findings is uncertain. Selective use of oral anticoagulants in patients with confirmed HALT and restricted leaflet motion with elevated gradients should be considered.

\subsubsection{HEART FAILURE}

Heart failure after valve surgery should lead to a quick search for SVD or PPM, deterioration of repair, LV dysfunction, or progression of another valve disease. Non-valvular-related causes such as CAD, hypertension, or sustained arrhythmias should also be considered. The management of patients with heart failure should follow the relevant guidelines and consensus documents. ${ }^{142,247}$

\section{Recommendations on management of prosthetic valve dysfunction}

\section{Recommendations}

Mechanical prosthetic thrombosis

Urgent or emergency valve replacement is recommended for obstructive thrombosis in critically ill patients without serious comorbidity. ${ }^{542}$

Fibrinolysis (using recombinant tissue plasminogen activator $10 \mathrm{mg}$ bolus $+90 \mathrm{mg}$ in 90 min with UFH or streptokinase $1500000 \mathrm{U}$ in 60 min without UFH) should be considered when surgery is not available or is very high risk, or for thrombosis of right-sided prostheses. ${ }^{542}$

Surgery should be considered for large $(>10 \mathrm{~mm})$ non-obstructive prosthetic thrombus complicated by embolism.

\section{Bioprosthetic thrombosis}

Anticoagulation using a VKA and/or UFH is recommended in bioprosthetic valve thrombosis before considering re-intervention.

Anticoagulation should be considered in patients with leaflet thickening and reduced leaflet motion leading to elevated gradients, at least until resolution. ${ }^{541,546}$

\section{Haemolysis and paravalvular leak}

Reoperation is recommended if a paravalvular leak is related to endocarditis or causes haemolysis requiring repeated blood transfusions or leading to severe heart failure symptoms.

Transcatheter closure should be considered for suitable paravalvular leaks with clinically significant regurgitation and/or haemolysis in patients at high or prohibitive surgical risk. ${ }^{547}$ 


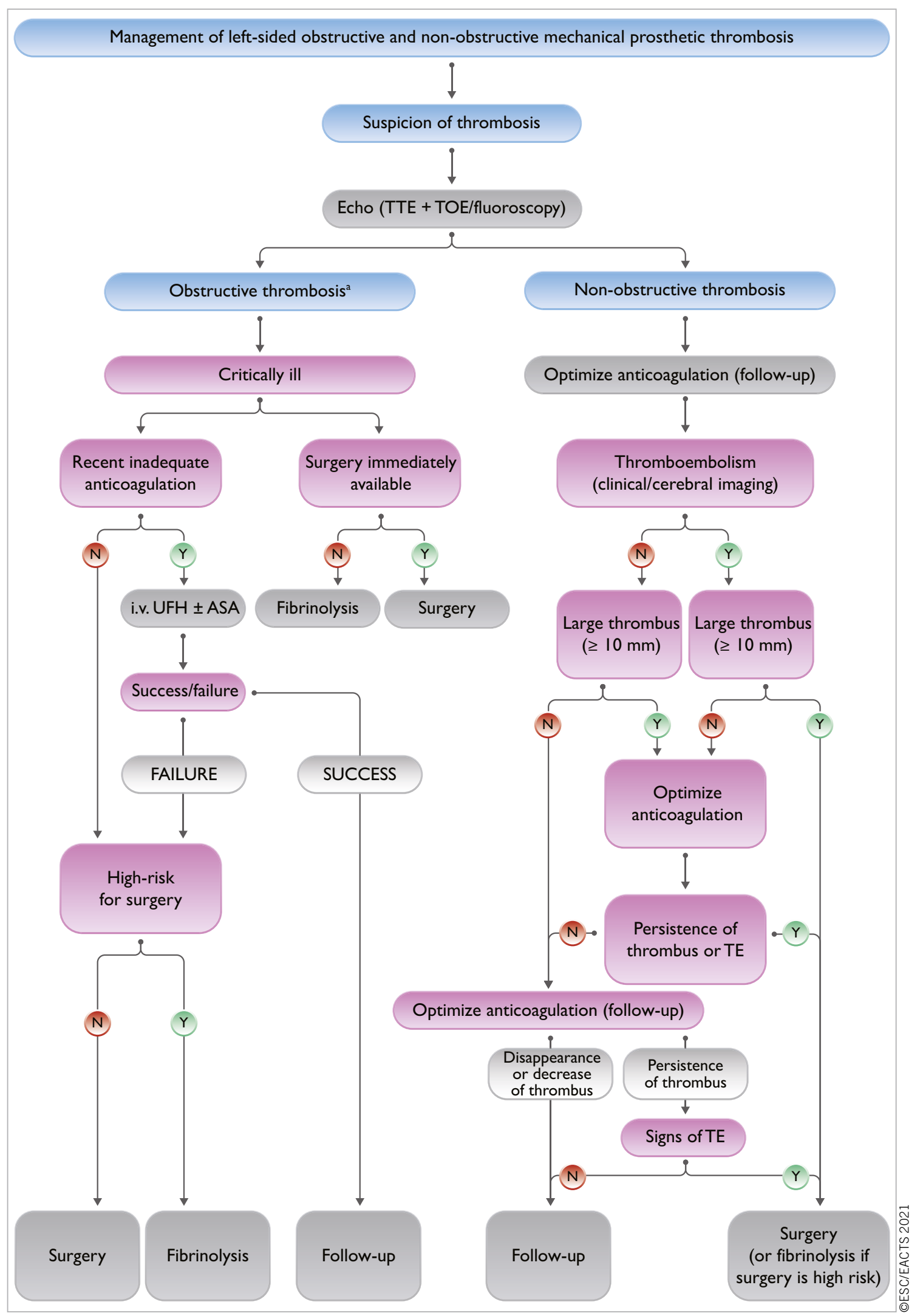

Figure 10. Management of left-sided obstructive and non-obstructive mechanical prosthetic thrombosis. ASA: acetylsalicylic acid; CCT: cardiac computed tomography; i.v.: intravenous; TOE: transoesophageal echocardiography; TE: thromboembolism;

TTE: transthoracic echocardiography; UFH: unfractionated heparin. Risk and benefits of both treatments should be individualized. The presence of a first-generation prosthesis is an incentive to surgery. ${ }^{a}$ Refer to recommendations for the imaging assessment of prosthetic heart valves. Evaluation generally includes TTE plus TOE or CCT and occasionally fluoroscopy. 


\begin{tabular}{|c|c|c|}
\hline $\begin{array}{l}\text { Decision on transcatheter or surgical closure of } \\
\text { clinically significant paravalvular leaks should be } \\
\text { considered based on patient risk status, leak } \\
\text { morphology, and local expertise. }\end{array}$ & Ila & c \\
\hline \multicolumn{3}{|l|}{ Bioprosthetic failure } \\
\hline $\begin{array}{l}\text { Reoperation is recommended in symptomatic } \\
\text { patients with a significant increase in } \\
\text { transprosthetic gradient (after exclusion of valve } \\
\text { thrombosis) or severe regurgitation. }\end{array}$ & I & c \\
\hline $\begin{array}{l}\text { Transcatheter, transfemoral valve-in-valve } \\
\text { implantation in the aortic position should be } \\
\text { considered by the Heart Team depending on } \\
\text { anatomic considerations, features of the } \\
\text { prosthesis, and in patients who are at high } \\
\text { operative risk or inoperable. }{ }^{529}\end{array}$ & Ila & B \\
\hline $\begin{array}{l}\text { Transcatheter valve-in-valve implantation in the } \\
\text { mitral and tricuspid position may be considered } \\
\text { in selected patients at high risk for surgical } \\
\text { reintervention. }{ }^{382,531,532}\end{array}$ & Ilb & B \\
\hline $\begin{array}{l}\text { Reoperation should be considered in } \\
\text { asymptomatic patients with significant } \\
\text { prosthetic dysfunction if reoperation is low risk. }\end{array}$ & Ila & C \\
\hline
\end{tabular}

UFH: unfractionated heparin; VKA: vitamin $\mathrm{K}$ antagonist. ${ }^{\mathrm{a}}$ Class of recommendation. ${ }^{b}$ Level of evidence.

\section{Management during non-cardiac surgery}

Cardiovascular morbidity and mortality are increased in patients with VHD who undergo NCS. Symptomatic severe aortic stenosis or mitral stenosis may require valve replacement or percutaneous intervention before NCS. A detailed description of recommendations in the setting is available in specific ESC Guidelines. ${ }^{489}$

\subsection{PREOPERATIVE EVALUATION}

Patient and surgical specific factors dictate the strategy. ${ }^{489,548,549}$ The cardiologist provides recommendations on pre- and perioperative management, surveillance, and continuation of chronic cardiovascular medical treatment. Echocardiography should be performed in any patient with VHD requiring NCS. Determination of functional capacity is a pivotal step in preoperative risk assessment, measured either by ability to perform activities in daily life or by exercise test. The decision for management should be taken after multidisciplinary discussion involving cardiologists, surgeons, and cardiac anaesthesiologists, as well as the team who will be in charge of NCS.

Patients receiving anticoagulation treatment should be managed as discussed in section 11 .

\subsection{SPECIFIC VALVE LESIONS}

\subsubsection{AORTIC STENOSIS}

In patients with severe aortic stenosis, urgent NCS should be performed under careful haemodynamic monitoring. In case of high risk of NCS, balloon valvuloplasty may be considered before NCS. ${ }^{549}$ Management related to elective NCS depends on the presence of symptoms and the type of surgery. ${ }^{489,549-553}$ In symptomatic patients, aortic valve procedure should be considered before NCS. The type of procedure, TAVI or SAVR, is decided by the Heart Team. In asymptomatic patients, elective NCS, if at low to moderate risk, can be performed safely, albeit with a risk of worsening heart failure. ${ }^{489,552,553}$ If NCS implies large volume shifts, aortic valve procedure (TAVI or SAVR) should be considered first according to the Heart Team's decision (Figure 11).

\subsubsection{MITRAL STENOSIS}

NCS can be performed safely in patients with non-significant mitral stenosis (valve area $>1.5 \mathrm{~cm}^{2}$ ) and in asymptomatic patients with significant mitral stenosis and an SPAP $<50 \mathrm{mmHg}$. In symptomatic patients or in patients with SPAP $>50 \mathrm{mmHg}$, correction of mitral stenosis, by means of PMC whenever possible, should be attempted before NCS if it is high risk.

\subsubsection{AORTIC AND MITRAL REGURGITATION}

NCS can be performed safely in asymptomatic patients with severe mitral regurgitation or aortic regurgitation and preserved LV function. The presence of symptoms or LV dysfunction should lead to consideration of valvular surgery, but this is seldom needed before NCS. If LV dysfunction is severe (ejection fraction $<30 \%$ ) and/or SPAP is $>50 / 60 \mathrm{mmHg}$, NCS should be performed only if strictly necessary and after optimization of medical therapy for heart failure.

\subsection{PERIOPERATIVE MONITORING}

Heart rate control (particularly in mitral stenosis) and careful fluid management (particularly in aortic stenosis) are needed. TOE monitoring may be considered.

\section{Management during pregnancy}

Detailed guidelines on the management of cardiovascular disease during pregnancy are available in another document. ${ }^{554}$ The decision for management before and during pregnancy should be taken after multidisciplinary discussion in the pregnancy Heart Team involving cardiologists, cardiac surgeons, obstetricians, neonatologists, and anaesthesiologists.

\subsection{MANAGEMENT BEFORE PREGNANCY}

Valve disease should be evaluated before pregnancy and treated if necessary. ${ }^{554,555}$

Pregnancy should be discouraged, and intervention should be recommended before pregnancy in the following cases:

- Patients with mitral stenosis and a valve area $<1.5 \mathrm{~cm}^{2}$ (especially if $\left.<1.0 \mathrm{~cm}^{2}\right) .554,556$

- All symptomatic patients with severe AS or asymptomatic patients with impaired LV function (LVEF $<50 \%$ ) or an abnormal exercise test should be counselled against pregnancy, and surgery should be performed pre-pregnancy. ${ }^{554,557}$

- Women with Marfan syndrome and an aortic diameter $>45 \mathrm{~mm}$ should be strongly discouraged from becoming pregnant without prior aortic repair because of the high risk of aortic dissection. Although an aortic diameter $<40 \mathrm{~mm}$ is rarely associated with aortic dissection, a completely safe diameter does not exist. With an aortic diameter between 40 and $45 \mathrm{~mm}$, previous aortic growth and family history are important for advising pregnancy with or without aortic repair. ${ }^{558}$ Although the actual risk of dissection is not well documented in the setting of bicuspid valves, 


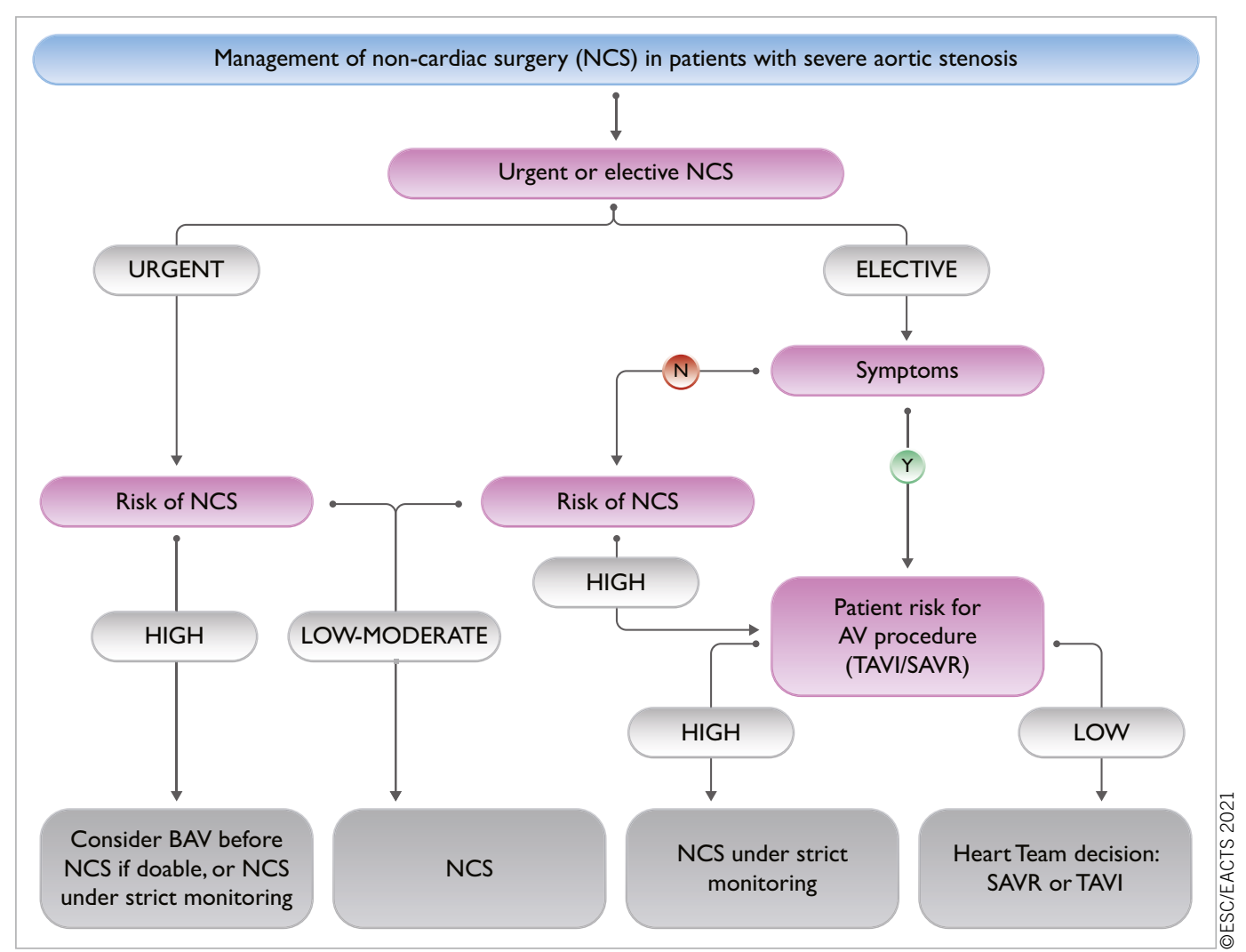

Figure 11. Management of non-cardiac surgery (NCS) in patients with severe aortic stenosis. AV: aortic valve; BAV: balloon aortic valvuloplasty; NCS: non cardiac surgery; SAVR: surgical aortic valve replacement; TAVI: transcatheter aortic valve implantation.

counselling against pregnancy is recommended in the setting of aortic diameters $>50 \mathrm{~mm}\left(>27 \mathrm{~mm}^{2}\right.$ BSA). ${ }^{559}$ Finally, an aortic diameter $>25 \mathrm{~mm} / \mathrm{m}^{2}$ BSA in Turner syndrome and all patients with vascular Ehlers-Danlos syndrome are also contraindications for pregnancy.

In women considering pregnancy and requiring heart valve replacement, it is recommended to choose the prosthesis in consultation with a pregnancy Heart Team. ${ }^{554,560}$

Pregnancy in women with a mechanical valve, especially in the mitral position, is associated with a high risk of maternal and foetal complications, ${ }^{554,561}$ which should be carefully discussed with the patient and family.

\subsection{MANAGEMENT DURING PREGNANCY}

\subsubsection{PATIENTS WITH NATIVE VALVE DISEASE}

Moderate or severe mitral stenosis with a valve area $<1.5 \mathrm{~cm}^{2}$ in pregnant women is usually poorly tolerated. PMC should be considered in severely symptomatic patients [New York Heart Association (NYHA) class III-IV] and/or those with SPAP $>50 \mathrm{mmHg}$ despite optimal therapy. PMC should preferably be performed after the 20th week of pregnancy in experienced centres. ${ }^{554}$

In patients who are severely symptomatic despite medical therapy, BAV for severe aortic stenosis can be undertaken by an experienced operator. ${ }^{557}$ TAVI is a promising alternative, but experience during pregnancy is very limited. ${ }^{554}$
Surgery under cardiopulmonary bypass is associated with a foetal mortality rate of $15-56 \%{ }^{562}$ and should be restricted to the rare conditions that threaten the mother's life if transcatheter intervention is not possible or has failed. Valve replacement should be considered after early delivery by caesarean section.

Caesarean section is recommended for patients with severe mitral or aortic stenosis, ascending aortic diameter $>45 \mathrm{~mm}$, severe pulmonary hypertension, or if delivery starts while treated with a VKA or $<2$ weeks after discontinuation of a VKA.

\subsubsection{MECHANICAL PROSTHESIS}

It is recommended to manage pregnancy in patients with MHV in a centre with a pregnancy Heart Team. ${ }^{554}$

Therapeutic anticoagulation during pregnancy is of utmost importance to avoid complications in these patients, keeping in mind that no anticoagulation regimen is ideal and management will require a careful balance between maternal and foetal risks.

In patients requiring $<5 \mathrm{mg} /$ day warfarin, oral anticoagulants throughout pregnancy and a change to UFH before delivery is favoured. In patients requiring higher doses, switching to LMWH during the first trimester with strict anti-Xa monitoring (therapeutic range $0.8-1.2 \mathrm{IU} / \mathrm{mL}$, aortic valve prosthesis; and 1.0-1.2 IU/mL, mitral and right sided valve prosthesis) and the use of oral anticoagulants afterwards is favoured with a change to UFH before delivery. ${ }^{554}$ 


\section{Key messages \\ GENERAL COMMENTS}

1. Precise evaluation of the patient's history and symptomatic status, as well as proper physical examination, are crucial for the diagnosis and management of VHD.

2. Echocardiography is the key technique to diagnose VHD and assess its severity and prognosis. Other non-invasive investigations such as CMR, CCT, fluoroscopy, and biomarkers provide important additional information in selected patients. Stress testing should be widely used in asymptomatic patients. Invasive investigation, beyond preoperative coronary angiography, is restricted to situations where non-invasive evaluation is inconclusive.

3. Decision making in elderly patients requires the integration of multiple parameters, including estimation of life expectancy and anticipated quality of life, evaluation of comorbidities, and general condition (including frailty).

4. Decision making in asymptomatic patients weighs the risk of intervention against the expected natural history of VHD. Stress testing should be liberally performed.

5. Informed patient's expectations and values are an important part of the decision-making process.

6. Interventions (surgery or transcatheter) are indicated in symptomatic patients (spontaneous or exercise induced) in the absence of futility. In selected asymptomatic patients, presence of predictors of rapid symptom progression justifies early intervention when procedural risk is low.

7. Heart Valve Centres with multidisciplinary Heart Teams, Heart Valve Clinics, comprehensive equipment, and sufficient volumes of procedures are required to deliver high-quality care and provide adequate training.

8. Careful follow-up of symptomatic status, LV/RV size, and function is mandatory in asymptomatic patients with severe VHD if an intervention is not yet indicated.

9. In patients with $\mathrm{AF}, \mathrm{NOACs}$ are contraindicated in patients with clinically significant mitral stenosis or mechanical valves. For stroke prevention in patients who are eligible for $\mathrm{OAC}$, NOACs are recommended in preference to VKAs in patients with aortic stenosis, aortic and mitral regurgitation, or aortic bioprostheses $>3$ months after implantation.

\section{AORTIC REGURGITATION}

10. The evaluation of aortic regurgitation requires careful assessment of potentially associated aortic dilatation to guide the timing and type of surgery.

\section{AORTIC STENOSIS}

11. Diagnosis of severe aortic stenosis requires integrative evaluation of pressure gradients (the most robust measurements), AVA, extent of valve calcification, flow conditions, and LV function.

12. Selection of the most appropriate mode of intervention by the Heart Team should take into account clinical characteristics (age and estimated life expectancy, general condition), anatomical characteristics, the relative risks of SAVR and TAVI, the feasibility of transfemoral TAVI, local experience and outcome data, as well as informed patient preference.

\section{MITRAL REGURGITATION}

13. Regarding imaging, routine quantification of EROA is an important part of the integrative evaluation for quantification and risk stratification in patients with PMR. 3D transoesophageal echocardiography is more accurate than 2D echocardiography for defining the underlying mechanism of PMR. CMR is useful when echocardiographic evaluation of severe PMR grade is inconclusive.

14. Surgical mitral valve repair is the preferred method of treatment in PMR if a durable repair can be achieved. TEER is a safe but less efficacious alternative that may be considered in patients with contraindications for surgery or high operative risk.

15. In patients with severe SMR, GDMT (including CRT if indicated) should be the first step. If the patient remains symptomatic: mitral surgery is recommended concomitantly in patients with an indication for $\mathrm{CABG}$ or other cardiac surgery. Isolated valve surgery may be considered in selected patients. TEER should be considered in patients not eligible for surgery and fulfilling criteria indicating an increased chance of responding to the treatment. Circulatory support devices, cardiac transplantation, or palliative care should be considered as an alternative in patients with end-stage LV and/or RV failure.

\section{MITRAL STENOSIS}

16. PMC is currently the standard of care in patients with severe rheumatic mitral stenosis and favourable valve anatomy.

17. Decision making as to the type of intervention used in patients with unfavourable anatomy is still a matter of debate and must take into account the multifactorial nature of predicting the results of PMC.

\section{TRICUSPID REGURGITATION}

18. Relevant tricuspid regurgitation requires early intervention to avoid secondary damage of the RV.

19. Tricuspid regurgitation should be liberally treated at the time of left-sided valve surgery. Isolated surgery of severe secondary tricuspid regurgitation (with or without previous left-sided valve surgery) requires comprehensive assessment of the underlying disease, pulmonary haemodynamics, and RV function.

\section{PROSTHETIC VALVES}

20. The choice between a mechanical prosthesis and a bioprosthesis should be patient-centred and multifactorial based on patient characteristics, the indication for lifelong anticoagulation, the potential and risks of a re-intervention, and the informed patient preference. 
21. Clinical assessment of prosthetic valves should be performed yearly and as soon as possible if new cardiac symptoms occur.

\section{Gaps in evidence}

Important gaps in evidence exist in the following aspects of VHD:

\section{GENERAL COMMENTS}

1. Prognostic value of CMR-derived indices in patients with aortic regurgitation, aortic stenosis, and mitral regurgitation.

2. Tools for risk stratification for the decision for intervention (including the avoidance of futile interventions) and the choice of the type of intervention (TAVI vs. SAVR for aortic stenosis, repair vs. replacement for mitral and aortic regurgitation).

3. In asymptomatic patients with aortic regurgitation, aortic stenosis, and mitral regurgitation, identification and evaluation of earlier markers of LV dysfunction (biomarkers, imaging, multimodality) as well as longitudinal and translational studies on progression.

4. Gender issues regarding pathophysiology, indications, and timing of treatment.

5. Minimum volumes of procedures that are required to achieve optimal results of intervention.

6. Safety and efficacy of NOACs in patients with surgical or transcatheter bioprostheses in the first 3 months after implantation.

7. Patient education for shared decision making and timely evaluation.

8. Systematic epidemiological data addressing the burden of rheumatic heart disease.

9. Advocacy of VHD.

\section{AORTIC REGURGITATION}

10. Potential differences in the risk of aortic complications depending on subtypes of aortic aneurysms (site and morphology), as well as in patients with bicuspid aortic valves.

11. Further evaluation of surgical aortic valve repair.

\section{AORTIC STENOSIS}

12. Pathophysiology of progression and novel therapeutic targets for medical treatment.

13. Further research to evaluate the role of intervention:

- a. Long-term durability of transcatheter heart valves in comparison with surgical bioprostheses.

- b. Role of intervention (SAVR or TAVI) in asymptomatic patients.

- c. Role of TAVI in younger low-risk patients, patients with aortic stenosis affecting bicuspid valves, and patients with moderate aortic stenosis and LV impairment.

- d. Results of re-intervention (valve or coronary) after TAVI or SAVR.
- e. The role of revascularization in patients with severe aortic stenosis and asymptomatic concomitant CAD.

\section{MITRAL REGURGITATION}

14. Association between PMR and sudden cardiac death and ventricular arrhythmias.

15. Role of genetic testing to mitral valve prolapse.

16. Further evaluation of the role of intervention:

- a. Long-term results of transcatheter intervention.

- b. Indications of transcatheter intervention in patients with severe PMR at lower surgical risk.

- c. Potential impact of mitral valve intervention (surgery and catheter intervention) on survival in patients with SMR.

- d. Selection of criteria to identify responders to TEER for SMR (severity criteria, concept of 'disproportionate mitral regurgitation').

- e. The role of newer transcatheter treatment options (annuloplasty, combined repair techniques, valve replacement).

\section{MITRAL STENOSIS}

17. Scores predicting the results and complications of PMC, particularly that of severe mitral regurgitation.

18. Role of transcatheter mitral valve implantation in high-risk patients, particularly in patients with severe degenerative mitral stenosis and MAC.

\section{TRICUSPID REGURGITATION}

19. Quantification of tricuspid regurgitation severity and evaluation of RV function.

20. Further research to evaluate the role of intervention:

- a. Criteria for optimal timing of surgery in primary tricuspid regurgitation.

- b. Evidence on the clinical impact, timing, and treatment modality of isolated severe secondary tricuspid regurgitation.

- c. Criteria for concomitant tricuspid valve surgery at the time of left-sided surgery in patients without severe tricuspid regurgitation.

- d. Results and indications of transcatheter tricuspid valve treatment.

\section{COMBINED AND MULTI-VALVE DISEASES}

21. Further evaluation of the impact on outcomes and modalities of transcatheter intervention to better define the indications for intervention.

\section{PREGNANCY}

22. Optimal management of pregnant women with MHVs regarding antithrombotic regimens.

\section{NON-CARDIAC SURGERY}

23. Evaluation of the role of 'urgent TAVI' in the management of patients with severe aortic stenosis undergoing NCS. 


\section{To Do and Not To Do}

\begin{tabular}{|l|l|}
\hline Recommendations & \multicolumn{1}{|l|}{ CI } \\
\hline Recommendations for management of CAD in patients with VHD \\
\hline Diagnosis of CAD \\
\hline Coronary angiography is recommended before valve surgery in patients with severe VHD and any of the following: \\
- History of cardiovascular disease. \\
- Suspected myocardial ischaemia. \\
- LV systolic dysfunction. \\
- In men >40 years of age and postmenopausal women. \\
- One or more cardiovascular risk factors. \\
\hline Coronary angiography is recommended in the evaluation of severe SMR. \\
\hline Indications for myocardial revascularization
\end{tabular}

$\mathrm{CABG}$ is recommended in patients with a primary indication for aortic/mitral/tricuspid valve surgery and coronary artery diameter stenosis $\geq 70 \%$.

Recommendations on management of atrial fibrillation in patients with native VHD

Anticoagulation

For stroke prevention in AF patients who are eligible for OAC, NOACs are recommended in preference to VKAs in patients with aortic stenosis, aortic and mitral regurgitation.

The use of NOACs is not recommended in patients with AF and moderate to severe mitral stenosis.

Recommendations on indications for surgery in (A) severe aortic regurgitation and (B) aortic root or tubular ascending aortic aneurysm (irrespective of the severity of aortic regurgitation)

A) Severe aortic regurgitation

Surgery is recommended in symptomatic patients regardless of LV function.

Surgery is recommended in asymptomatic patients with LVESD $>50 \mathrm{~mm}$ or LVESD $>25 \mathrm{~mm} / \mathrm{m}^{2}$ BSA (in patients with small body size) or resting LVEF $\leq 50 \%$.

Surgery is recommended in symptomatic and asymptomatic patients with severe aortic regurgitation undergoing CABG or surgery of the ascending aorta or of another valve.

B) Aortic root or tubular ascending aortic aneurysm (irrespective of the severity of aortic regurgitation)

Valve-sparing aortic root replacement is recommended in young patients with aortic root dilation, if performed in experienced centres and durable results are expected.

Ascending aortic surgery is indicated in patients with Marfan syndrome who have aortic root disease with a maximal ascending aortic diameter $\geq 50 \mathrm{~mm}$

Recommendations on indications for intervention in symptomatic (A) and asymptomatic (B) aortic stenosis and recommended mode of intervention (C)

\section{A) Symptomatic aortic stenosis}

Intervention is recommended in symptomatic patients with severe, high-gradient aortic stenosis [mean gradient $\geq 40 \mathrm{mmHg}$, peak velocity $\geq 4.0 \mathrm{~m} / \mathrm{s}$ and valve area $\leq 1.0 \mathrm{~cm}^{2}$ (or $\left.\left.\leq 0.6 \mathrm{~cm}^{2} / \mathrm{m}^{2}\right)\right]$.

Intervention is recommended in symptomatic patients with severe low-flow (SVi $\left.\leq 35 \mathrm{~mL} / \mathrm{m}^{2}\right)$, low-gradient $(<40 \mathrm{mmHg})$ aortic stenosis with reduced ejection fraction $(<50 \%)$ and evidence of flow (contractile) reserve.

Intervention is not recommended in patients with severe comorbidities when the intervention is unlikely to improve quality of life or prolong survival $>1$ year.

\section{B) Asymptomatic patients with severe aortic stenosis}

Intervention is recommended in asymptomatic patients with severe aortic stenosis and systolic LV dysfunction (LVEF <50\%) without another cause.

Intervention is recommended in asymptomatic patients with severe aortic stenosis and demonstrable symptoms on exercise testing.

\section{C) Mode of intervention}

Aortic valve interventions must be performed in Heart Valve Centres that declare their local expertise and outcomes data, have active interventional cardiology and cardiac surgical programmes on site, and a structured collaborative Heart Team approach.

The choice between surgical and transcatheter intervention must be based upon careful evaluation of clinical, anatomical, and procedural factors by the Heart Team, weighing the risks and benefits of each approach for an individual patient. The Heart Team recommendation should be discussed with the patient who can then make an informed treatment choice.

SAVR is recommended in younger patients who are low risk for surgery ( $<75$ years and STS-PROM/ EuroSCORE II $<4 \%)$, or in patients who are operable and unsuitable for transfemoral TAVI. 
TAVI is recommended in older patients ( $\geq 75$ years), or in those who are high risk (STS-PROM/EuroSCORE II $>8 \%$ ) or unsuitable for surgery.

SAVR or TAVI are recommended for remaining patients according to individual clinical, anatomical, and procedural characteristics.

\section{D) Concomitant aortic valve surgery at the time of other cardiac/ascending aorta surgery}

SAVR is recommended in patients with severe aortic stenosis undergoing CABG or surgical intervention on the ascending aorta or another valve.

Recommendations on indications for intervention in severe primary mitral regurgitation

Mitral valve repair is the recommended surgical technique when the results are expected to be durable.

Surgery is recommended in symptomatic patients who are operable and not high risk.

Surgery is recommended in asymptomatic patients with LV dysfunction (LVESD $\geq 40 \mathrm{~mm}$ and/or LVEF $\leq 60 \%$ ).

Recommendations on indications for mitral valve intervention in chronic severe secondary mitral regurgitation

Valve surgery/intervention is recommended only in patients with severe SMR who remain symptomatic despite GDMT

(including CRT if indicated) and has to be decided by a structured collaborative Heart Team.

Patients with concomitant coronary artery or other cardiac disease requiring treatment

Valve surgery is recommended in patients undergoing CABG or other cardiac surgery.

Recommendations on indications for percutaneous mitral commissurotomy and mitral valve surgery in clinically significant

(moderate or severe) mitral stenosis (valve area $\leq 1.5 \mathrm{~cm} 2$ )

PMC is recommended in symptomatic patients without unfavourable characteristics for PMC.

PMC is recommended in any symptomatic patients with a contraindication or a high risk for surgery.

Mitral valve surgery is recommended in symptomatic patients who are not suitable for PMC in the absence of futility.

Recommendations on indications for intervention in tricuspid valve disease

Recommendations on tricuspid stenosis

Surgery is recommended in symptomatic patients with severe tricuspid stenosis.

Surgery is recommended in patients with severe tricuspid stenosis undergoing left-sided valve intervention.

Recommendations on primary tricuspid regurgitation

Surgery is recommended in patients with severe primary tricuspid regurgitation undergoing left-sided valve surgery.

Surgery is recommended in symptomatic patients with isolated severe primary tricuspid regurgitation without severe RV dysfunction.

Recommendations on secondary tricuspid regurgitation

Surgery is recommended in patients with severe secondary tricuspid regurgitation undergoing left-sided valve surgery.

Recommendations for prosthetic valve selection

Mechanical prostheses

A mechanical prosthesis is recommended according to the desire of the informed patient and if there are no contraindications to long-term anticoagulation.

A mechanical prosthesis is recommended in patients at risk of accelerated SVD.

Biological prostheses

A bioprosthesis is recommended according to the desire of the informed patient.

A bioprosthesis is recommended when good-quality anticoagulation is unlikely (adherence problems, not readily available), contraindicated because of high bleeding risk (previous major bleed, comorbidities, unwillingness, adherence problems, lifestyle, occupation), and in those patients whose life expectancy is lower than the presumed durability of the bioprosthesis.

A bioprosthesis is recommended in case of reoperation for mechanical valve thrombosis despite good long-term anticoagulant control.

Recommendations for perioperative and postoperative antithrombotic management of valve replacement or repair Management of antithrombotic therapy in the perioperative period

It is recommended that VKAs are timely discontinued prior to elective surgery to aim for an INR $<1.5$.

Bridging of OAC, when interruption is needed, is recommended in patients with any of the following indications:

- Mechanical prosthetic heart valve.

- AF with significant mitral stenosis.

- AF with a $\mathrm{CHA}_{2} \mathrm{DS}_{2}$-VASc score $\geq 3$ for women or 2 for men.

- Acute thrombotic event within the previous 4 weeks.

- High acute thromboembolic risk.

Therapeutic doses of either UFH or subcutaneous LMWH are recommended for bridging.

In patients with MHVs, it is recommended to (re)-initiate the VKA on the first postoperative day. 


\begin{tabular}{|c|c|c|}
\hline $\begin{array}{l}\text { In patients who have undergone valve surgery with an indication for postoperative therapeutic bridging, it is recommended } \\
\text { to start either UFH or LMWH 12-24 h after surgery. }\end{array}$ & I & C \\
\hline $\begin{array}{l}\text { In patients undergoing surgery, it is recommended that aspirin therapy, if indicated, is maintained during the periprocedural } \\
\text { period. }\end{array}$ & I & C \\
\hline $\begin{array}{l}\text { In patients treated with DAPT after recent } \mathrm{PCI} \text { (within } 1 \text { month) who need to undergo heart valve surgery in the absence of } \\
\text { an indication for } \mathrm{OAC} \text {, it is recommended to resume the } \mathrm{P}_{12} \mathrm{Y}_{12} \text { inhibitor postoperatively as soon as there is no concern over } \\
\text { bleeding. }\end{array}$ & I & C \\
\hline \multicolumn{3}{|l|}{ Patients with an indication to concomitant antiplatelet therapy } \\
\hline $\begin{array}{l}\text { After uncomplicated } \mathrm{PCI} \text { or ACS in patients requiring long-term } \mathrm{OAC} \text {, early cessation ( } \leq 1 \text { week) of aspirin and continuation } \\
\text { of dual therapy with } \mathrm{OAC} \text { and a } \mathrm{P} 2 \mathrm{Y}_{12} \text { inhibitor (preferably clopidogrel) for up to } 6 \text { months (or up to } 12 \text { months in ACS) is } \\
\text { recommended if the risk of stent thrombosis is low or if concerns about bleeding risk prevail over concerns about risk of } \\
\text { stent thrombosis, irrespective of the type of stent used. }\end{array}$ & $\mathbf{I}$ & B \\
\hline Discontinuation of antiplatelet treatment in patients treated with an OAC is recommended after 12 months. & $\mathbf{I}$ & B \\
\hline \multicolumn{3}{|l|}{ Surgical valve replacement } \\
\hline OAC using a VKA is recommended lifelong for all patients with a MHV prosthesis. & $\mathbf{I}$ & B \\
\hline $\begin{array}{l}\text { For patients with a VKA, INR self-management is recommended provided appropriate training and quality control are } \\
\text { performed. }\end{array}$ & I & B \\
\hline $\begin{array}{l}\text { OAC is recommended for patients undergoing implantation of a surgical BHV who have other indications for } \\
\text { anticoagulation. }\end{array}$ & I & C \\
\hline NOACs are not recommended in patients with an MHV. & III & B \\
\hline \multicolumn{3}{|l|}{ Transcatheter aortic valve implantation } \\
\hline OAC is recommended lifelong for TAVI patients who have other indications for anticoagulation. & $\mathbf{I}$ & B \\
\hline Lifelong SAPT is recommended after TAVI in patients with no baseline indication for OAC. & $\mathbf{I}$ & A \\
\hline Routine use of OAC is not recommended after TAVI in patients who have no baseline indication for OAC. & III & B \\
\hline \multicolumn{3}{|l|}{ Recommendations on management of prosthetic valve dysfunction } \\
\hline \multicolumn{3}{|l|}{ Mechanical prosthetic thrombosis } \\
\hline $\begin{array}{l}\text { Urgent or emergency valve replacement is recommended for obstructive thrombosis in critically ill patients without serious } \\
\text { comorbidity. }\end{array}$ & $\mathbf{I}$ & B \\
\hline \multicolumn{3}{|l|}{ Bioprosthetic thrombosis } \\
\hline $\begin{array}{l}\text { Anticoagulation using a VKA and/or UFH is recommended in bioprosthetic valve thrombosis before considering } \\
\text { reintervention. }\end{array}$ & $\mathbf{I}$ & C \\
\hline \multicolumn{3}{|l|}{ Haemolysis and paravalvular leak } \\
\hline $\begin{array}{l}\text { Reoperation is recommended if a paravalvular leak is related to endocarditis or causes haemolysis requ } \\
\text { transfusions or leading to severe heart failure symptoms. }\end{array}$ & I & C \\
\hline \multicolumn{3}{|l|}{ Bioprosthetic failure } \\
\hline $\begin{array}{l}\text { Reoperation is recommended in symptomatic patients with a significant increase in transpr } \\
\text { of valve thrombosis) or severe regurgitation. }\end{array}$ & I & C \\
\hline
\end{tabular}

ACS: acute coronary syndrome; AF: atrial fibrillation; BHV: biological heart valve; BSA: body surface area; CABG: coronary artery bypass grafting; CAD: coronary artery disease; CRT: cardiac resynchronization therapy; DAPT: dual antiplatelet therapy; EuroSCORE: European System for Cardiac Operative Risk Evaluation; GDMT: guidelinedirected medical therapy; h: hours; INR: international normalized ratio; LMWH: low-molecular-weight heparin; LV: left ventricle/left ventricular; LVEF: left ventricular ejection fraction; LVESD: left ventricular end-systolic diameter; MHV: mechanical heart valve; MR: mitral regurgitation; NOAC: non-vitamin K antagonist oral anticoagulant; OAC: oral anticoagulation; PCI: percutaneous coronary intervention; PMC: percutaneous mitral commissurotomy; RV: right ventricle/right ventricular; SAPT: single antiplatelet therapy; SAVR: surgical aortic valve replacement; SMR: secondary mitral regurgitation; STS-PROM: Society of Thoracic Surgeons - predicted risk of mortality; SVD: structural valve deterioration; SVi: stroke volume index; TAVI: transcatheter aortic valve implantation; UFH: unfractionated heparin; VHD: valvular heart disease; VKA: vitamin $\mathrm{K}$ antagonist.

\section{Supplementary data}

Supplementary Table 1. Cardiovascular and non-cardiovascular factors linked with transcatheter aortic valve implantation-related futility featured within the PARTNER and FRANCE 2 transcatheter aortic valve implantation-risk score models.

Supplementary Table 2. Katz Index of Independence in Activities of Daily Living.

Supplementary Table 3. Essential frailty toolset in older adults undergoing aortic valve replacement.
Supplementary Table 4. Medical comorbidities and factors predicting poorer outcomes post transcatheter aortic valve implantation.

Supplementary Table 5. Integrated approach for estimating transcatheter aortic valve implantation-specific risk and futility.

Supplementary Table 6. Risk-of-bias judgments for all evaluated trials.

Supplementary Table 7. Main inclusion/exclusion criteria suggesting an increased chance of responding to TEER in patients with SMR. 
Supplementary Table 8. Echocardiographic scores used for assessing the feasibility of percutaneous mitral commissurotomy: Wilkins score, Cormier score, and Echo score 'Revisited'.

Supplementary Figure 1. Criteria for patients selection for MitraClip procedure.

Supplementary Figure 2. Peri- and post-procedural management of antithrombotic therapy in patients with indication to $\mathrm{OAC}$ and ACS/PCI.

Supplementary Figure 3. Management of OAC in patients with an indication for preoperative bridging.

The supplementary data are published online at:

https://eurointervention.pcronline.com/

doi/10.4244/EIJ-E-21-00009

\section{Author information}

Author/Task Force Member Affiliations: Fabien Praz, Department of Cardiology, Bern University Hospital, University of Bern, Bern, Switzerland; Milan Milojevic ${ }^{1}$, Department of Cardiac Surgery and Cardiovascular Research, Dedinje Cardiovascular Institute, Belgrade, Serbia and Department of Cardiothoracic Surgery, Erasmus University Medical Center, Rotterdam, Netherlands; Stephan Baldus, Heart Centre, Department of Cardiology, University Hospital Cologne, Cologne, Germany; Johann Bauersachs, Department of Cardiology and Angiology, Hannover Medical School, Hannover, Germany; Davide Capodanno, Division of Cardiology, Azienda Ospedaliero Universitaria Policlinico 'Gaspare Rodolico - San Marco', Catania, Italy; Lenard Conradi ${ }^{1}$, Department of Cardiovascular Surgery, University Heart and Vascular Centre Hamburg, Hamburg, Germany; Michele De Bonis ${ }^{1}$, Department of Cardiac Surgery, IRCCS San Raffaele Hospital, 'Vita-Salute' San Raffaele University, Milan, Italy; Ruggero De Paulis ${ }^{1}$, Cardiac Surgery Department, European Hospital, Unicamillus International Medical University, Rome, Italy; Victoria Delgado, Cardiology, Leiden University Medical Centre, Leiden, Netherlands; Nick Freemantle ${ }^{1}$, Institute of Clinical Trials and Methodology, University College London, London, United Kingdom; Martine Gilard, Cardiology, Brest University Hospital, Brest, France; Kristina H. Haugaa, Department of Cardiology, Oslo University Hospital and University of Oslo, Oslo, Norway; Anders Jeppsson ${ }^{1}$, Department of Cardiothoracic Surgery, Sahlgrenska University Hospital, Gothenburg, Sweden; Peter Jüni, Applied Health Research Centre (AHRC), Li Ka Shing Knowledge Institute of St. Michael's Hospital, Department of Medicine and Institute of Health Policy, Management and Evaluation, University of Toronto, Canada; Luc Pierard, Cardiology, University of Liège, Liège, Belgium; Bernard D. Prendergast, Department of Cardiology, St Thomas' Hospital and Cleveland Clinic London, London, United Kingdom; J. Rafael Sádaba ${ }^{1}$, Department of Cardiac Surgery, Complejo Hospitalario de Navarra, Pamplona, Spain; Christophe Tribouilloy, Department of Cardiology, Amiens University Hospital, Amiens, France; Wojtek Wojakowski, Cardiology and Structural Heart Diseases, Medical University of Silesia, Katowice, Poland.

\section{Appendix}

\section{ESC/EACTS Scientific Document Group}

Includes Document Reviewers and ESC National Cardiac Societies.

Document Reviewers: Franz-Josef Neumann (ESC Review Coordinator) (Germany), Patrick Myers ${ }^{1}$ (EACTS Review Coordinator) (Switzerland), Magdy Abdelhamid (Egypt), Stephan Achenbach (Germany), Riccardo Asteggiano (Italy), Fabio Barili ${ }^{1}$ (Italy), Michael A. Borger (Germany), Thierry Carrel $^{1}$ (Switzerland), JeanPhilippe Collet (France), Dan Foldager (Denmark), Gilbert Habib (France), Christian Hassager (Denmark), Alar Irs ${ }^{1}$ (Estonia), Bernard Iung (France), Marjan Jahangiri ${ }^{1}$ (United Kingdom), Hugo A. Katus (Germany), Konstantinos C. Koskinas (Switzerland), Steffen Massberg (Germany), Christian E. Mueller (Switzerland), Jens Cosedis Nielsen (Denmark), Philippe Pibarot (Canada), Amina Rakisheva (Kazakhstan), Marco Roffi (Switzerland), Andrea Rubboli (Italy), Evgeny Shlyakhto (Russia), Matthias Siepe' (Germany), Marta Sitges (Spain), Lars Sondergaard (Denmark), Miguel SousaUva $^{1}$ (Portugal), Guiseppe Tarantini (Italy), Jose Luis Zamorano (Spain).

ESC National Cardiac Societies actively involved in the review process of the 2021 ESC/EACTS Guidelines for the management of valvular heart disease.

Algeria: Algerian Society of Cardiology, Yasmina Benchabi; Armenia: Armenian Cardiologists Association, Aram Chilingaryan; Austria: Austrian Society of Cardiology, Bernhard Metzler; Azerbaijan: Azerbaijan Society of Cardiology, Yasmin Rustamova; Belarus: Belorussian Scientific Society of Cardiologists, Vadim Shumavets; Belgium: Belgian Society of Cardiology, Patrizio Lancellotti; Bosnia and Herzegovina: Association of Cardiologists of Bosnia and Herzegovina, Elnur Smajic; Bulgaria: Bulgarian Society of Cardiology, Diana Trendafilova-Lazarova; Croatia: Croatian Cardiac Society, Jure Samardzic; Cyprus: Cyprus Society of Cardiology, Maria Karakyriou; Czech Republic: Czech Society of Cardiology, Tomáš Paleček; Denmark: Danish Society of Cardiology, Jordi Sanchez Dahl; Egypt: Egyptian Society of Cardiology, Marwa Sayed Meshaal; Estonia: Estonian Society of Cardiology, Kairit Palm; Finland: Finnish Cardiac Society, Marko Virtanen; France: French Society of Cardiology, Claire Bouleti; Georgia: Georgian Society of Cardiology, Zviad Bakhutashvili; Germany: German Cardiac Society, Stephan Achenbach; Greece: Hellenic Society of Cardiology, Maria Boutsikou; Hungary: Hungarian Society of Cardiology, Attila Béla Kertész; Iceland: Icelandic Society of Cardiology, Ragnar Danielsen; Israel: Israel Heart Society, Yan Topilsky; Italy: Italian Federation of Cardiology, Paolo Golino; Kazakhstan: Association of Cardiologists of Kazakhstan, Rustem Tuleutayev; Kosovo (Republic of): Kosovo Society of Cardiology, Shpend Elezi; Kyrgyzstan: Kyrgyz Society of Cardiology, Alina Kerimkulova; Latvia: Latvian Society of Cardiology, Ainars Rudzitis; Lithuania: Lithuanian Society of Cardiology, Sigita Glaveckaite; Luxembourg: Luxembourg Society of Cardiology, Rouguiatou Sow; Malta: Maltese Cardiac Society, Daniela Cassar Demarco; Montenegro: Montenegro Society of Cardiology, Nebojsa Bulatovic; Morocco: Moroccan Society 
of Cardiology, Aicha Aouad; Netherlands: Netherlands Society of Cardiology, Renée van den Brink; North Macedonia: North Macedonian Society of Cardiology, Emilija Antova; Norway: Norwegian Society of Cardiology, Jan Otto Beitnes; Poland: Polish Cardiac Society, Andrzej Ochala; Portugal: Portuguese Society of Cardiology, Regina Ribeiras; Romania: Romanian Society of Cardiology, Dragos Vinereanu; Russian Federation: Russian Society of Cardiology, Olga Irtyuga; Serbia: Cardiology Society of Serbia, Branislava Ivanovic; Slovakia: Slovak Society of Cardiology, Iveta Simkova; Spain: Spanish Society of Cardiology, Ariana González Gómez; Sweden: Swedish Society of Cardiology, Giovanna Sarno; Switzerland: Swiss Society of Cardiology, Giovanni B. Pedrazzini; Syrian Arab Republic: Syrian Cardiovascular Association, Walid Bsata; Tunisia: Tunisian Society of Cardiology and Cardio-Vascular Surgery, Lilia Zakhama; Turkey: Turkish Society of Cardiology, Levent Korkmaz; Ukraine: Ukrainian Association of Cardiology, Sergii Cherniuk; United Kingdom of Great Britain and Northern Ireland: British Cardiovascular Society, Mohammed Y. Khanji; Uzbekistan: Association of Cardiologists of Uzbekistan, Islamjan Sharipov. ESC Clinical Practice Guidelines Committee (CPG): Colin Baigent (Chairperson) (United Kingdom), Magdy Abdelhamid (Egypt), Victor Aboyans (France), Sotiris Antoniou (United Kingdom), Elena Arbelo (Spain), Riccardo Asteggiano (Italy), Andreas Baumbach (United Kingdom), Michael A. Borger (Germany), Jelena Čelutkienè (Lithuania), Maja Cikes (Croatia), Jean-Philippe Collet (France), Volkmar Falk (Germany), Laurent Fauchier (France), Chris P. Gale (United Kingdom), Sigrun Halvorsen (Norway), Bernard Iung (France), Tiny Jaarsma (Sweden), Aleksandra Konradi (Russia), Konstantinos C. Koskinas (Switzerland), Dipak Kotecha (United Kingdom), Ulf Landmesser (Germany), Basil S. Lewis (Israel), Ales Linhart (Czech Republic), Maja-Lisa Løchen (Norway), Lis Neubeck (United Kingdom), Jens Cosedis Nielsen (Denmark), Steffen E. Petersen (United Kingdom), Eva Prescott (Denmark), Amina Rakisheva (Kazakhstan), Marta Sitges (Spain), Rhian M. Touyz (United Kingdom).

EACTS Council: Friedhelm Beyersdorf(Germany), Lorenzo Galletti (Italy), Mark Hazekamp (Netherlands), Peter Licht (Denmark), Patrick Myers (Switzerland), Patrick Perier (Germany), Richard Prager (United States of America), Eric Roessner (Germany), J. Rafael Sádaba (Spain), Matthias Siepe (Germany), Konstantinos Tsagakis (Germany), Alicja Zientara (United Kingdom).

\section{References}

1. Iung B, Delgado V, Rosenhek R, Price S, Prendergast B, Wendler O, De Bonis M, Tribouilloy C, Evangelista A, Bogachev-Prokophiev A, Apor A, Ince H, Laroche C, Popescu BA, Pierard L, Haude M, Hindricks G, Ruschitzka F, Windecker S, Bax JJ, Maggioni A, Vahanian A, EORP VHD II Investigators. Contemporary presentation and management of valvular heart disease: The EURObservational Research Programme Valvular Heart Disease II Survey. Circulation. 2019;140:1156-1169.

2. Yadgir S, Johnson CO, Aboyans V, Adebayo OM, Adedoyin RA, Afarideh M, Alahdab F, Alashi A, Alipour V, Arabloo J, Azari S, Barthelemy CM, Benziger CP, Berman AE, Bijani A, Carrero JJ, Carvalho F, Daryani A, Duraes AR, Esteghamati A, Farid TA, Farzadfar F, Fernandes E, Filip I, Gad MM, Hamidi S, Hay SI, Ilesanmi OS, Naghibi Irvani SS, Jurisson M, Kasaeian A, Kengne AP, Khan AR, Kisa A, Kisa S, Kolte D, Manafi N, Manafi A, Mensah GA, Mirrakhimov EM, Mohammad Y, Mokdad AH, Negoi RI, Thi Nguyen HL, Nguyen TH, Nixon MR, Otto CM, Patel S, Pilgrim T, Radfar A, Rawaf DL, Rawaf S, Rawasia WF, Rezapour A, Roever L,
Saad AM, Saadatagah S, Senthilkumaran S, Sliwa K, Tesfay BE, Tran BX, Ullah I, Vaduganathan M, Vasankari TJ, Wolfe CDA, Yonemoto N, Roth GA, Global Burden of Disease Study Nonrheumatic Valve Disease Collaborators. Global, regional, and national burden of calcific aortic valve and degenerative mitral valve diseases, 19902017. Circulation. 2020;141:1670-1680.

3. Cahill TJ, Prothero A, Wilson J, Kennedy A, Brubert J, Masters M, Newton JD, Dawkins S, Enriquez-Sarano M, Prendergast BD, Myerson SG. Community prevalence, mechanisms and outcome of mitral or tricuspid regurgitation. Heart 2021 doi: 10.1136/heartjnl-2020-318482.

4. Habib G, Lancellotti P, Antunes MJ, Bongiorni MG, Casalta JP, Del Zotti F, Dulgheru R, El Khoury G, Erba PA, Iung B, Miro JM, Mulder BJ, Plonska-Gosciniak E, Price S, Roos-Hesselink J, Snygg-Martin U, Thuny F, Tornos Mas P, Vilacosta I, Zamorano JL, ESC Scientific Document Group. 2015 ESC Guidelines for the management of infective endocarditis: The Task Force for the Management of Infective Endocarditis of the European Society of Cardiology (ESC). Endorsed by: European Association for Cardio-Thoracic Surgery (EACTS), the European Association of Nuclear Medicine (EANM). Eur Heart J. 2015;36:3075-3128.

5. Baumgartner H, De Backer J, Babu-Narayan SV, Budts W, Chessa M, Diller GP, Lung B, Kluin J, Lang IM, Meijboom F, Moons P, Mulder BJM, Oechslin E, RoosHesselink JW, Schwerzmann M, Sondergaard L, Zeppenfeld K, ESC Scientific Document Group. 2020 ESC Guidelines for the management of adult congenital heart disease. Eur Heart J. 2021;42:563-645.

6. Pelliccia A, Sharma S, Gati S, Back M, Borjesson M, Caselli S, Collet JP, Corrado D, Drezner JA, Halle M, Hansen D, Heidbuchel H, Myers J, Niebauer J, Papadakis M, Piepoli MF, Prescott E, Roos-Hesselink JW, Graham Stuart A, Taylor RS, Thompson PD, Tiberi M, Vanhees L, Wilhelm M, ESC Scientific Document Group. 2020 ESC Guidelines on sports cardiology and exercise in patients with cardiovascular disease. Eur Heart J. 2021;42:17-96.

7. Agricola E, Ancona F, Brochet E, Donal E, Dweck M, Faletra F, Lancellotti P, Mahmoud-Elsayed H, Marsan NA, Maurovich-Hovart P, Monaghan M, Ribeiro J, Sade LE, Swaans M, Von Bardeleben RS, Wunderlich N, Zamorano JL, Popescu BA, Cosyns B, Edvardsen T. The structural heart disease interventional imager rationale, skills and training: a position paper of the European Association of Cardiovascular Imaging. Eur Heart J Cardiovasc Imaging. 2021;22:471-479.

8. Hahn RT, Mahmood F, Kodali S, Lang R, Monaghan M, Gillam LD, Swaminathan M, Bonow RO, von Bardeleben RS, Bax JJ, Grayburn P, Zoghbi WA, Sengupta PP, Chandrashekhar Y, Little SH. Core competencies in echocardiography for imaging structural heart disease interventions: an expert consensus statement. JACC Cardiovasc Imaging. 2019;12:2560-2570.

9. Lancellotti P, Magne J, Dulgheru R, Clavel MA, Donal E, Vannan MA, Chambers J, Rosenhek R, Habib G, Lloyd G, Nistri S, Garbi M, Marchetta S, Fattouch K, Coisne A, Montaigne D, Modine T, Davin L, Gach O, Radermecker M, Liu S, Gillam L, Rossi A, Galli E, Ilardi F, Tastet L, Capoulade R, Zilberszac R, Vollema EM, Delgado V, Cosyns B, Lafitte S, Bernard A, Pierard LA, Bax JJ, Pibarot P, Oury C. Outcomes of patients with asymptomatic aortic stenosis followed up in heart valve clinics. JAMA Cardiol. 2018;3:1060-1068.

10. Lancellotti P, Rosenhek R, Pibarot P, Iung B, Otto CM, Tornos P, Donal E, Prendergast B, Magne J, La Canna G, Pierard LA, Maurer G. ESC Working Group on Valvular Heart Disease position paper-heart valve clinics: organization, structure, and experiences. Eur Heart J. 2013;34:1597-1606.

11. Chambers JB, Prendergast B, Iung B, Rosenhek R, Zamorano JL, Pierard LA, Modine T, Falk V, Kappetein AP, Pibarot P, Sundt T, Baumgartner H, Bax JJ, Lancellotti P. Standards defining a 'Heart Valve Centre': ESC Working Group on Valvular Heart Disease and European Association for Cardiothoracic Surgery Viewpoint. Eur Heart J. 2017;38:2177-2183.

12. Badheka AO, Patel NJ, Panaich SS, Patel SV, Jhamnani S, Singh V, Pant S, Patel N, Patel N, Arora S, Thakkar B, Manvar S, Dhoble A, Patel A, Savani C, Patel J, Chothani A, Savani GT, Deshmukh A, Grines CL, Curtis J, Mangi AA, Cleman M, Forrest JK. Effect of hospital volume on outcomes of transcatheter aortic valve implantation. Am J Cardiol. 2015;116:587-594.

13. Nishimura RA, O'Gara PT, Bavaria JE, Brindis RG, Carroll JD, Kavinsky CJ, Lindman BR, Linderbaum JA, Little SH, Mack MJ, Mauri L, Miranda WR, Shahian DM, Sundt TM 3rd. 2019 AATS/ACC/ASE/SCAI/STS Expert Consensus Systems of Care Document: a proposal to optimize care for patients with valvular heart disease: a joint report of the American Association for Thoracic Surgery, American College of Cardiology, American Society of Echocardiography, Society for Cardiovascular Angiography and Interventions, and Society of Thoracic Surgeons. J Am Coll Cardiol. 2019;73:2609-2635.

14. Chhatriwalla AK, Vemulapalli S, Szerlip M, Kodali S, Hahn RT, Saxon JT, Mack MJ, Ailawadi G, Rymer J, Manandhar P, Kosinski AS, Sorajja P. Operator experience and outcomes of transcatheter mitral valve repair in the United States. J Am Coll Cardiol. 2019;74:2955-2965. 
15. Timmis A, Townsend N, Gale CP, Torbica A, Lettino M, Petersen SE, Mossialos EA, Maggioni AP, Kazakiewicz D, May HT, De Smedt D, Flather M, Zuhlke L, Beltrame JF, Huculeci R, Tavazzi L, Hindricks G, Bax J, Casadei B, Achenbach S, Wright L, Vardas P, European Society of Cardiology. European Society of Cardiology: cardiovascular disease statistics 2019. Eur Heart J. 2020;41:12-85.

16. Vemulapalli S, Carroll JD, Mack MJ, Li Z, Dai D, Kosinski AS, Kumbhani DJ, Ruiz CE, Thourani VH, Hanzel G, Gleason TG, Herrmann HC, Brindis RG, Bavaria JE. Procedural volume and outcomes for transcatheter aortic-valve replacement. $N$ Engl $J$ Med. 2019;380:2541-2550.

17. Mao J, Redberg RF, Carroll JD, Marinac-Dabic D, Laschinger J, Thourani V, Mack M, Sedrakyan A. Association between hospital surgical aortic valve replacement volume and transcatheter aortic valve replacement outcomes. JAMA Cardiol. 2018; 3:1070-1078.

18. Bonow RO, O'Gara PT, Adams DH, Badhwar V, Bavaria JE, Elmariah S, Hung JW, Lindenfeld J, Morris A, Satpathy R, Whisenant B, Woo YJ. 2019 AATS/ACC/SCAI/ STS Expert Consensus Systems of Care Document: operator and institutional recommendations and requirements for transcatheter mitral valve intervention: a joint report of the American Association for Thoracic Surgery, the American College of Cardiology, the Society for Cardiovascular Angiography and Interventions, and The Society of Thoracic Surgeons. J Am Coll Cardiol. 2020;76:96-117.

19. Garbi M, Chambers J, Pierard L, Maisano F, Lancellotti P. Heart Valve Specialist Core Syllabus: a learning framework for continuous medical education on valvular heart disease. European Society of Cardiology, 1 June 2021. https://www.escardio.org/ Councils/Council-on-Valvular-Heart-Disease/heart-valve-specialist-core-syllabus. Accessed on 20 July 2021.

20. Dreyfus G, Windecker S. How to shape the future of cardiology and cardiac surgery? Eur Heart J. 2020;41:3693-3701.

21. Iung B, Delgado V, Lazure P, Murray S, Sirnes PA, Rosenhek R, Price S, Metra M, Carrera C, De Bonis M, Haude M, Hindricks G, Bax J, Vahanian A. Educational needs and application of guidelines in the management of patients with mitral regurgitation. A European mixed-methods study. Eur Heart J. 2018;39:1295-1303.

22. Popescu BA, Andrade MJ, Badano LP, Fox KF, Flachskampf FA, Lancellotti P, Varga A, Sicari R, Evangelista A, Nihoyannopoulos P, Zamorano JL European Association of E, Document R Derumeaux G, Kasprzak JD, Roelandt JR. European Association of Echocardiography recommendations for training, competence, and quality improvement in echocardiography. Eur J Echocardiogr. 2009;10:893-905.

23. Chambers JB, Garbi M, Nieman K, Myerson S, Pierard LA, Habib G, Zamorano JL, Edvardsen T, Lancellotti P This document was reviewed by members of the ESDC Delgado V, Cosyns B, Donal E, Dulgheru R, Galderisi M, Lombardi M, Muraru D, Kauffmann P, Cardim N, Haugaa K, Rosenhek R. Appropriateness criteria for the use of cardiovascular imaging in heart valve disease in adults: a European Association of Cardiovascular Imaging report of literature review and current practice. Eur Heart $J$ Cardiovasc Imaging. 2017;18:489-498.

24. Lancellotti P, Tribouilloy C, Hagendorff A, Popescu BA, Edvardsen T, Pierard LA, Badano L, Zamorano JL, Scientific Document Committee of the European Association of Cardiovascular Imaging. Recommendations for the echocardiographic assessment of native valvular regurgitation: an executive summary from the European Association of Cardiovascular Imaging. Eur Heart J Cardiovasc Imaging. 2013;14:611-644.

25. Baumgartner HC, Hung JC-C, Bermejo J, Chambers JB, Edvardsen T, Goldstein S, Lancellotti P, LeFevre M, Miller F Jr., Otto CM. Recommendations on the echocardiographic assessment of aortic valve stenosis: a focused update from the European Association of Cardiovascular Imaging and the American Society of Echocardiography. Eur Heart J Cardiovasc Imaging. 2017;18:254-275.

26. Magne J, Cosyns B, Popescu BA, Carstensen HG, Dahl J, Desai MY, Kearney L, Lancellotti P, Marwick TH, Sato K, Takeuchi M, Zito C, Casalta AC, Mohty D, Pierard L, Habib G, Donal E. Distribution and prognostic significance of left ventricular global longitudinal strain in asymptomatic significant aortic stenosis: an individual participant data meta-analysis. JACC Cardiovasc Imaging. 2019;12:84-92.

27. Prihadi EA, van der Bijl P, Dietz M, Abou R, Vollema EM, Marsan NA, Delgado V, Bax JJ. Prognostic implications of right ventricular free wall longitudinal strain in patients with significant functional tricuspid regurgitation. Circ Cardiovasc Imaging. 2019;12: e008666.

28. van Rosendael PJ, van Wijngaarden SE, Kamperidis V, Kong WKF, Leung M, Ajmone Marsan N, Delgado V, Bax JJ. Integrated imaging of echocardiography and computed tomography to grade mitral regurgitation severity in patients undergoing transcatheter aortic valve implantation. Eur Heart J. 2017;38:2221-2226.

29. Schulz E, Tamm A, Kasper-Konig W, Beiras-Fernandez A, Vahl CF, Munzel T, von Bardeleben RS. Transapical implantation of a transcatheter aortic valve prosthesis into a mitral annuloplasty ring guided by real-time three-dimensional cardiac computed tomography-fluoroscopy fusion imaging. Eur Heart J. 2018;39:327-328.

30. Henri C, Pierard LA, Lancellotti P, Mongeon FP, Pibarot P, Basmadjian AJ. Exercise testing and stress imaging in valvular heart disease. Can $J$ Cardiol. 2014;30:1012-1026.
31. Picano E, Pibarot P, Lancellotti P, Monin JL, Bonow RO. The emerging role of exercise testing and stress echocardiography in valvular heart disease. $J \mathrm{Am} \mathrm{Coll}$ Cardiol. 2009;54:2251-2260.

32. Monin JL, Quere JP, Monchi M, Petit H, Baleynaud S, Chauvel C, Pop C, Ohlmann P, Lelguen C, Dehant P, Tribouilloy C, Gueret P. Low-gradient aortic stenosis: operative risk stratification and predictors for long-term outcome: a multicenter study using dobutamine stress hemodynamics. Circulation. 2003;108:319-324.

33. Bing R, Cavalcante JL, Everett RJ, Clavel MA, Newby DE, Dweck MR. Imaging and impact of myocardial fibrosis in aortic stenosis. JACC Cardiovasc Imaging. 2019;12:283-296.

34. American College of Cardiology Foundation Task Force on Expert Consensus D, Hundley WG, Bluemke DA, Finn JP, Flamm SD, Fogel MA, Friedrich MG, Ho VB, Jerosch-Herold M, Kramer CM, Manning WJ, Patel M, Pohost GM, Stillman AE, White RD, Woodard PK., ACCF/ACR/AHA/NASCI/SCMR 2010 expert consensus document on cardiovascular magnetic resonance: a report of the American College of Cardiology Foundation Task Force on Expert Consensus Documents. J Am Coll Cardiol. 2010;55:2614-2662.

35. Cueff C, Serfaty JM, Cimadevilla C, Laissy JP, Himbert D, Tubach F, Duval X, Iung B, Enriquez-Sarano M, Vahanian A, Messika-Zeitoun D. Measurement of aortic valve calcification using multislice computed tomography: correlation with haemodynamic severity of aortic stenosis and clinical implication for patients with low ejection fraction. Heart. 2011;97:721-726.

36. Clavel MA, Messika-Zeitoun D, Pibarot P, Aggarwal SR, Malouf J, Araoz PA, Michelena HI, Cueff C, Larose E, Capoulade R, Vahanian A, Enriquez-Sarano M. The complex nature of discordant severe calcified aortic valve disease grading: new insights from combined Doppler echocardiographic and computed tomographic study. J Am Coll Cardiol. 2013;62:2329-2338.

37. Pibarot P, Magne J, Leipsic J, Cote N, Blanke P, Thourani VH, Hahn R. Imaging for predicting and assessing prosthesis-patient mismatch after aortic valve replacement. JACC Cardiovasc Imaging. 2019;12:149-162.

38. Pulerwitz TC, Khalique OK, Leb J, Hahn RT, Nazif TM, Leon MB, George I, Vahl TP, D'Souza B, Bapat VN, Dumeer S, Kodali SK, Einstein AJ. Optimizing cardiac CT protocols for comprehensive acquisition prior to percutaneous MV and TV repair/replacement. JACC Cardiovasc Imaging. 2020;13:836-850.

39. San S, Ravis E, Tessonier L, Philip M, Cammilleri S, Lavagna F, Norscini G, Arregle F, Martel H, Oliver L, Torras O, Renard S, Ambrosi P, Camoin L, Casalta AC, Hubert S, Casalta JP, Gouriet F, Riberi A, Avierinos JF, Lepidi H, Collart F, Raoult D, Drancourt M, Habib G. Prognostic value of (18)F-fluorodeoxyglucose positron emission tomography/computed tomography in infective endocarditis. $\mathrm{J} \mathrm{Am} \mathrm{Coll} \mathrm{Cardiol.}$ 2019;74:1031-1040.

40. Habib G, Erba PA, Iung B, Donal E, Cosyns B, Laroche C, Popescu BA, Prendergast B, Tornos P, Sadeghpour A, Oliver L, Vaskelyte JJ, Sow R, Axler O, Maggioni AP, Lancellotti P, EURO-ENDO Investigators. Clinical presentation, aetiology and outcome of infective endocarditis. Results of the ESC-EORP EURO-ENDO (European infective endocarditis) registry: a prospective cohort study. Eur Heart $J$. 2019;40:3222-3232.

41. Clavel MA, Tribouilloy C, Vanoverschelde JL, Pizarro R, Suri RM, Szymanski C, Lazam S, Oberti P, Michelena HI, Jaffe A, Enriquez-Sarano M. Association of B-type natriuretic peptide with survival in patients with degenerative mitral regurgitation. J Am Coll Cardiol. 2016;68:1297-1307.

42. Lindman BR, Clavel MA, Abu-Alhayja'a R, Cote N, Dagenais F, Novak E, Voisine P, Poulin A, Arsenault BJ, Desmeules P, Dahou A, Taster L, Aldahoun K, Bosse Y, Mathieu P, Pibarot P. Multimarker approach to identify patients with higher mortality and rehospitalization rate after surgical aortic valve replacement for aortic stenosis. JACC Cardiovasc Interv. 2018;11:2172-2181.

43. Tastet L, Tribouilloy C, Marechaux S, Vollema EM, Delgado V, Salaun E, Shen M, Capoulade R, Clavel MA, Arsenault M, Bedard E, Bernier M, Beaudoin J, Narula J, Lancellotti P, Bax JJ, Genereux P, Pibarot P. Staging cardiac damage in patients with asymptomatic aortic valve stenosis. J Am Coll Cardiol. 2019;74:550-563.

44. Genereux P, Pibarot P, Redfors B, Mack MJ, Makkar RR, Jaber WA, Svensson LG, Kapadia S, Tuzcu EM, Thourani VH, Babaliaros V, Herrmann HC, Szeto WY, Cohen DJ, Lindman BR, McAndrew T, Alu MC, Douglas PS, Hahn RT, Kodali SK, Smith CR, Miller DC, Webb JG, Leon MB. Staging classification of aortic stenosis based on the extent of cardiac damage. Eur Heart J. 2017;38:3351-3358.

45. Authors/Task Force m Windecker S, Kolh P, Alfonso F, Collet JP, Cremer J, Falk V, Filippatos G, Hamm C, Head SJ, Juni P, Kappetein AP, Kastrati A, Knuuti J, Landmesser U, Laufer G, Neumann FJ, Richter DJ, Schauerte P, Sousa Uva M, Stefanini GG, Taggart DP, Torracca L, Valgimigli M, Wijns W, Witkowski A. 2014 ESC/EACTS Guidelines on myocardial revascularization: The Task Force on Myocardial Revascularization of the European Society of Cardiology (ESC) and the European Association for Cardio-Thoracic Surgery (EACTS). Developed with the special contribution of the European Association of Percutaneous Cardiovascular Interventions (EAPCI). Eur Heart J. 2014;35:2541-2619. 
46. Knuuti J, Wijns W, Saraste A, Capodanno D, Barbato E, Funck-Brentano C, Prescott E, Storey RF, Deaton C, Cuisset T, Agewall S, Dickstein K, Edvardsen T, Escaned J, Gersh BJ, Svitil P, Gilard M, Hasdai D, Hatala R, Mahfoud F, Masip J, Muneretto C, Valgimigli M, Achenbach S, Bax JJ, ESC Scientific Document Group. 2019 ESC Guidelines for the diagnosis and management of chronic coronary syndromes. Eur Heart J. 2020;41:407-477.

47. Scarsini R, Pesarini G, Zivelonghi C, Piccoli A, Ferrero V, Lunardi M, Gottin L, Zanetti C, Faggian G, Ribichini F. Physiologic evaluation of coronary lesions using instantaneous wave-free ratio (iFR) in patients with severe aortic stenosis undergoing transcatheter aortic valve implantation. EuroIntervention. 2018;13:1512-1519.

48. Scarsini R, Pesarini G, Zivelonghi C, Piccoli A, Ferrero V, Lunardi M, Barbierato M, Caprioglio F, Vassanelli C, Ribichini F. Coronary physiology in patients with severe aortic stenosis: comparison between fractional flow reserve and instantaneous wavefree ratio. Int $J$ Cardiol. 2017;243:40-46.

49. Osnabrugge RL, Speir AM, Head SJ, Fonner CE, Fonner E, Kappetein AP, Rich JB. Performance of EuroSCORE II in a large US database: implications for transcatheter aortic valve implantation. Eur J Cardiothorac Surg. 2014;46:400-408; discussion 408. 50. Barili F, Pacini D, Capo A, Rasovic O, Grossi C, Alamanni F, Di Bartolomeo R, Parolari A. Does EuroSCORE II perform better than its original versions? A multicentre validation study. Eur Heart J. 2013;34:22-29.

51. Provenchere S, Chevalier A, Ghodbane W, Bouleti C, Montravers P, Longrois D, Iung B. Is the EuroSCORE II reliable to estimate operative mortality among octogenarians? PLoS One. 2017;12: e0187056.

52. Iung B, Laouenan C, Himbert D, Eltchaninoff H, Chevreul K, Donzeau-Gouge P, Fajadet J, Leprince P, Leguerrier A, Lievre M, Prat A, Teiger E, Laskar M, Vahanian A, Gilard M, FRANCE 2 Investigators. Predictive factors of early mortality after transcatheter aortic valve implantation: individual risk assessment using a simple score. Heart. 2014;100:1016-1023.

53. Edwards FH, Cohen DJ, O'Brien SM, Peterson ED, Mack MJ, Shahian DM, Grover FL, Tuzcu EM, Thourani VH, Carroll J, Brennan JM, Brindis RG, Rumsfeld J, Holmes DR Jr., Steering Committee of the Society of Thoracic Surgeons/American College of Cardiology Transcatheter Valve Therapy R. Development and validation of a risk prediction model for in-hospital mortality after transcatheter aortic valve replacement. JAMA Cardiol. 2016;1:46-52.

54. Arnold SV, Reynolds MR, Lei Y, Magnuson EA, Kirtane AJ, Kodali SK, Zajarias A, Thourani VH, Green P, Rodes-Cabau J, Beohar N, Mack MJ, Leon MB, Cohen DJ, PARTNER Investigators. Predictors of poor outcomes after transcatheter aortic valve replacement: results from the PARTNER (Placement of Aortic Transcatheter Valve) trial. Circulation. 2014;129:2682-2690.

55. Afilalo J. The Clinical Frailty Scale: Upgrade your eyeball test. Circulation. 2017; 135:2025-2027.

56. Kundi H, Popma JJ, Reynolds MR, Strom JB, Pinto DS, Valsdottir LR, Shen C, Choi E, Yeh RW. Frailty and related outcomes in patients undergoing transcatheter valve therapies in a nationwide cohort. Eur Heart J. 2019;40:2231-2239.

57. Hosler QP, Maltagliati AJ, Shi SM, Afilalo J, Popma JJ, Khabbaz KR, Laham RJ, Guibone K, Kim DH. A practical two-stage frailty assessment for older adults undergoing aortic valve replacement. J Am Geriatr Soc. 2019;67:2031-2037.

58. Dent E, Martin FC, Bergman H, Woo J, Romero-Ortuno R, Walston JD. Management of frailty: opportunities, challenges, and future directions. Lancet. 2019;394:1376-1386.

59. Katz S. Assessing self-maintenance: activities of daily living, mobility, and instrumental activities of daily living. J Am Geriatr Soc. 1983;31:721-727.

60. Afilalo J, Lauck S, Kim DH, Lefevre T, Piazza N, Lachapelle K, Martucci G, Lamy A, Labinaz M, Peterson MD, Arora RC, Noiseux N, Rassi A, Palacios IF, Genereux P, Lindman BR, Asgar AW, Kim CA, Trnkus A, Morais JA, Langlois Y, Rudski LG, Morin JF, Popma JJ, Webb JG, Perrault LP. Frailty in older adults undergoing aortic valve replacement: the FRAILTY-AVR study. J Am Coll Cardiol. 2017; 70:689-700

61. Goldfarb M, Lauck S, Webb JG, Asgar AW, Perrault LP, Piazza N, Martucci G, Lachapelle K, Noiseux N, Kim DH, Popma JJ, Lefevre T, Labinaz M, Lamy A, Peterson MD, Arora RC, Morais JA, Morin JF, Rudski LG, Afilalo J. Malnutrition and mortality in frail and non-frail older adults undergoing aortic valve replacement. Circulation. 2018;138:2202-2211.

62. Yanagisawa R, Tanaka M, Yashima F, Arai T, Kohno T, Shimizu H, Fukuda K, Naganuma T, Mizutani K, Araki M, Tada N, Yamanaka F, Shirai S, Tabata M, Ueno H, Takagi K, Higashimori A, Watanabe Y, Yamamoto M, Hayashida K. Frequency and consequences of cognitive impairment in patients underwent transcatheter aortic valve implantation. Am J Cardiol. 2018;122:844-850.

63. Puri R, Iung B, Cohen DJ, Rodes-Cabau J. TAVI or no TAVI: identifying patients unlikely to benefit from transcatheter aortic valve implantation. Eur Heart $J$. 2016;37:2217-2225.

64. Gunter RL, Kilgo P, Guyton RA, Chen EP, Puskas JD, Cooper WA, Halkos ME, Lattouf OM, Babaliaros V, Myung R, Leshnower B, Thourani VH. Impact of preoperative chronic lung disease on survival after surgical aortic valve replacement Ann Thorac Surg. 2013;96:1322-1328.

65. Allende R, Webb JG, Munoz-Garcia AJ, de Jaegere P, Tamburino C, Dager AE, Cheema A, Serra V, Amat-Santos I, Velianou JL, Barbanti M, Dvir D, AlonsoBriales JH, Nuis RJ, Faqiri E, Imme S, Benitez LM, Cucalon AM, Al Lawati H, Garcia del Blanco B, Lopez J, Natarajan MK, DeLarochelliere R, Urena M, Ribeiro HB, Dumont E, Nombela-Franco L, Rodes-Cabau J. Advanced chronic kidney disease in patients undergoing transcatheter aortic valve implantation: insights on clinical outcomes and prognostic markers from a large cohort of patients. Eur Heart J. 2014 $35: 2685-2696$.

66. Tirado-Conte G, Rodes-Cabau J, Rodriguez-Olivares R, Barbanti M, Lhermusier T, Amat-Santos I, Toggweiler S, Cheema AN, Munoz-Garcia AJ, Serra V, Giordana F, Veiga G, Jimenez-Quevedo P, Campelo-Parada F, Loretz L, Todaro D, Del Trigo M, Hernandez-Garcia JM, Garcia Del Blanco B, Bruno F, de la Torre Hernandez JM, Stella P, Tamburino C, Macaya C, Nombela-Franco L. Clinical outcomes and prognosis markers of patients with liver disease undergoing transcatheter aortic valve replacement: a propensity score-matched analysis. Circ Cardiovasc Interv. 2018;11: e005727. 67. Abramowitz Y, Kazuno Y, Chakravarty T, Kawamori H, Maeno Y, Anderson D Allison Z, Mangat G, Cheng W, Gopal A, Jilaihawi H, Mack MJ, Makkar RR. Concomitant mitral annular calcification and severe aortic stenosis: prevalence, characteristics and outcome following transcatheter aortic valve replacement. Eur Heart J. 2017;38:1194-1203.

68. Lindman BR, Arnold SV, Bagur R, Clarke L, Coylewright M, Evans F, Hung J, Lauck SB, Peschin S, Sachdev V, Tate LM, Wasfy JH, Otto CM. Priorities for patientcentered research in valvular heart disease: a report from the National Heart, Lung, and Blood Institute Working Group. J Am Heart Assoc. 2020;9: e015975.

69. Hejjaji V, Cohen DJ, Carroll JD, Li Z, Manandhar P, Vemulapalli S, Nelson AJ, Malik AO, Mack MJ, Spertus JA, Arnold SV. Practical application of patient-reported health status measures for transcatheter valve therapies: insights from the Society of Thoracic Surgeons/American College of Cardiology Transcatheter Valve Therapies Registry. Circ Cardiovasc Qual Outcomes. 2021;14: e007187.

70. Steiner JM, Cooper S, Kirkpatrick JN. Palliative care in end-stage valvular heart disease. Heart. 2017;103:1233-1237.

71. Timmis A, Gale CP, Flather M, Maniadakis N, Vardas P. Cardiovascular disease statistics from the European atlas: inequalities between high- and middle-income member countries of the ESC. Eur Heart J Qual Care Clin Outcomes. 2018;4:1-3.

72. Neumann FJ, Sousa-Uva M, Ahlsson A, Alfonso F, Banning AP, Benedetto U, Byrne RA, Collet JP, Falk V, Head SJ, Juni P, Kastrati A, Koller A, Kristensen SD, Niebauer J, Richter DJ, Seferovic PM, Sibbing D, Stefanini GG, Windecker S, Yadav R, Zembala MO, ESC Scientific Document Group. 2018 ESC/EACTS Guidelines on myocardial revascularization. Eur Heart J. 2019;40:87-165.

73. Mach F, Baigent C, Catapano AL, Koskinas KC, Casula M, Badimon L, Chapman MJ, De Backer GG, Delgado V, Ference BA, Graham IM, Halliday A, Landmesser U, Mihaylova B, Pedersen TR, Riccardi G, Richter DJ, Sabatine MS, Taskinen MR, Tokgozoglu L, Wiklund O, ESC Scientific Document Group. 2019 ESC EAS Guidelines for the management of dyslipidaemias: lipid modification to reduce cardiovascular risk. Eur Heart J. 2020;41:111-188.

74. Hindricks G, Potpara T, Dagres N, Arbelo E, Bax JJ, Blomstrom-Lundqvist C, Boriani G, Castella M, Dan GA, Dilaveris PE, Fauchier L, Filippatos G, Kalman JM, La Meir M, Lane DA, Lebeau JP, Lettino M, Lip GYH, Pinto FJ, Thomas GN, Valgimigli M, Van Gelder IC, Van Putte BP, Watkins CL, ESC Scientific Documen Group. 2020 ESC Guidelines for the diagnosis and management of atrial fibrillation developed in collaboration with the European Association for Cardio-Thoracic Surgery (EACTS): The Task Force for the diagnosis and management of atrial fibrillation of the European Society of Cardiology (ESC). Developed with the special contribution of the European Heart Rhythm Association (EHRA) of the ESC. Eur Heart J. 2021;42: 373-498

75. Breithardt G, Baumgartner H, Berkowitz SD, Hellkamp AS, Piccini JP, Stevens SR, Lokhnygina Y, Patel MR, Halperin JL, Singer DE, Hankey GJ, Hacke W, Becker RC, Nessel CC, Mahaffey KW, Fox KA, Califf RM, Committee RAS, Investigators. Clinical characteristics and outcomes with rivaroxaban vs. warfarin in patients with non-valvular atrial fibrillation but underlying native mitral and aortic valve disease participating in the ROCKET AF trial. Eur Heart J. 2014;35:3377-3385.

76. Avezum A, Lopes RD, Schulte PJ, Lanas F, Gersh BJ, Hanna M, Pais P, Erol C, Diaz R, Bahit MC, Bartunek J, De Caterina R, Goto S, Ruzyllo W, Zhu J, Granger CB, Alexander JH. Apixaban in comparison with warfarin in patients with atrial fibrillation and valvular heart disease: findings from the Apixaban for Reduction in Stroke and Other Thromboembolic Events in Atrial Fibrillation (ARISTOTLE) trial. Circulation. 2015;132:624-632.

77. Ezekowitz MD, Nagarakanti R, Noack H, Brueckmann M, Litherland C, Jacobs M, Clemens A, Reilly PA, Connolly SJ, Yusuf S, Wallentin L. Comparison of dabigatran and warfarin in patients with atrial fibrillation and valvular heart disease: the RE-LY Trial (Randomized Evaluation of Long-Term Anticoagulant Therapy). Circulation. 2016;134:589-598 
78. De Caterina R, Renda G, Carnicelli AP, Nordio F, Trevisan M, Mercuri MF, Ruff CT, Antman EM, Braunwald E, Giugliano RP. Valvular heart disease patients on edoxaban or warfarin in the ENGAGE AF-TIMI 48 trial. $J$ Am Coll Cardiol. 2017;69:1372-1382

79. Rankin JS, Grau-Sepulveda MV, Ad N, Damiano RJ Jr., Gillinov AM, Brennan JM, McCarthy PM, Thourani VH, Jacobs JP, Shahian DM, Badhwar V. Associations between surgical ablation and operative mortality after mitral valve procedures. Ann Thorac Surg. 2018;105:1790-1796.

80. Tsai YC, Phan K, Munkholm-Larsen S, Tian DH, La Meir M, Yan TD. Surgical left atrial appendage occlusion during cardiac surgery for patients with atrial fibrillation: a meta-analysis. Eur J Cardiothorac Surg. 2015;47:847-854.

81. Martin Gutierrez E, Castano M, Gualis J, Martinez-Comendador JM, Maiorano P, Castillo L, Laguna G. Beneficial effect of left atrial appendage closure during cardiac surgery: a meta-analysis of 280585 patients. Eur J Cardiothorac Surg. 2020;57: 252-262.

82. Whitlock RP, Belley-Cote EP, Paparella D, Healey JS, Brady K, Sharma M, Reents W, Budera P, Baddour AJ, Fila P, Devereaux PJ, Bogachev-Prokophiev A, Boening A, Teoh KHT, Tagarakis GI, Slaughter MS, Royse AG, McGuinness S, Alings M, Punjabi PP, Mazer CD, Folkeringa RJ, Colli A, Avezum A, Nakamya J, Balasubramanian K, Vincent J, Voisine P, Lamy A, Yusuf S, Connolly SJ, LAAOS III Investigators. Left atrial appendage occlusion during cardiac surgery to prevent stroke. N Engl J Med. 2021;384:2081-2091.

83. RuffCT, Giugliano RP, Braunwald E, Hoffman EB, Deenadayalu N, Ezekowitz MD, Camm AJ, Weitz JI, Lewis BS, Parkhomenko A, Yamashita T, Antman EM. Comparison of the efficacy and safety of new oral anticoagulants with warfarin in patients with atrial fibrillation: a meta-analysis of randomised trials. Lancet. 2014;383:955-962.

84. Wang KL, Lip GY, Lin SJ, Chiang CE. Non-vitamin K antagonist oral anticoagulants for stroke prevention in Asian patients with nonvalvular atrial fibrillation: metaanalysis. Stroke. 2015;46:2555-2561.

85. Badhwar V, Rankin JS, Ad N, Grau-Sepulveda M, Damiano RJ, Gillinov AM, McCarthy PM, Thourani VH, Suri RM, Jacobs JP, Cox JL. Surgical ablation of atrial fibrillation in the United States: trends and propensity matched outcomes. Ann Thorac Surg. 2017; 104:493-500.

86. Gillinov AM, Gelijns AC, Parides MK, DeRose JJ Jr., Moskowitz AJ, Voisine P, Ailawadi G, Bouchard D, Smith PK, Mack MJ, Acker MA, Mullen JC, Rose EA Chang HL, Puskas JD, Couderc JP, Gardner TJ, Varghese R, Horvath KA, Bolling SF, Michler RE, Geller NL, Ascheim DD, Miller MA, Bagiella E, Moquete EG, Williams P, Taddei-Peters WC, O'Gara PT, Blackstone EH, Argenziano M, Investigators C. Surgical ablation of atrial fibrillation during mitral-valve surgery. $N$ Engl J Med. 2015;372:1399-1409.

87. Huffman MD, Karmali KN, Berendsen MA, Andrei AC, Kruse J, McCarthy PM, Malaisrie SC. Concomitant atrial fibrillation surgery for people undergoing cardiac surgery. Cochrane Database Syst Rev 2016: CD011814.

88. Wang H, Han J, Wang Z, Yin Z, Liu Z, Jin Y, Han H. A prospective randomized trial of the cut-and-sew Maze procedure in patients undergoing surgery for rheumatic mitral valve disease. J Thorac Cardiovasc Surg. 2018;155:608-617.

89. Lawrance CP, Henn MC, Miller JR, Sinn LA, Schuessler RB, Maniar HS, Damiano RJ, Jr. A minimally invasive Cox maze IV procedure is as effective as sternotomy while decreasing major morbidity and hospital stay. J Thorac Cardiovasc Surg. 2014;148:955-961.

90. Weimar T, Schena S, Bailey MS, Maniar HS, Schuessler RB, Cox JL, Damiano RJ, Jr. The Cox-Maze procedure for lone atrial fibrillation: a single-center experience over 2 decades. Circ Arrhythm Electrophysiol. 2012;5:8-14.

91. Karki P, Uranw S, Bastola S, Mahato R, Shrestha NR, Sherpa K, Dhungana S, Odutayo A, Gurung K, Pandey N, Agrawal K, Shah P, Rothenbuhler M, Juni P, Pilgrim T. Effectiveness of systematic echocardiographic screening for rheumatic heart disease in Nepalese schoolchildren: a cluster randomized clinical trial. JAMA Cardiol. 2021;6:420-426.

92. Gerber MA, Baltimore RS, Eaton CB, Gewitz M, Rowley AH, Shulman ST, Taubert KA. Prevention of rheumatic fever and diagnosis and treatment of acute Streptococcal pharyngitis: a scientific statement from the American Heart Association Rheumatic Fever, Endocarditis, and Kawasaki Disease Committee of the Council on Cardiovascular Disease in the Young, the Interdisciplinary Council on Functional Genomics and Translational Biology, and the Interdisciplinary Council on Quality of Care and Outcomes Research: endorsed by the American Academy of Pediatrics. Circulation. 2009;119:1541-1551.

93. Remenyi B, Carapetis J, Wyber R, Taubert K, Mayosi BM, World Heart Federation. Position statement of the World Heart Federation on the prevention and control of rheumatic heart disease. Nat Rev Cardiol. 2013;10:284-292.

94. Watkins DA, Beaton AZ, Carapetis JR, Karthikeyan G, Mayosi BM, Wyber R, Yacoub MH, Zuhlke LJ. Rheumatic heart disease worldwide: JACC Scientific Expert Panel. J Am Coll Cardiol. 2018;72:1397-1416.
95. Kumar RK, Antunes MJ, Beaton A, Mirabel M, Nkomo VT, Okello E, Regmi PR, Remenyi B, Sliwa-Hahnle K, Zuhlke LJ, Sable C, American Heart Association Council on Lifelong Congenital Heart Disease and Heart Health in the Young; Council on Cardiovascular and Stroke Nursing; and Council on Clinical Cardiology. Contemporary diagnosis and management of rheumatic heart disease: implications for closing the gap: a scientific statement from the American Heart Association. Circulation. 2020;142: e337-e357.

96. le Polain de Waroux JB, Pouleur AC, Goffinet C, Vancraeynest D, Van Dyck M, Robert A, Gerber BL, Pasquet A, El Khoury G, Vanoverschelde JL. Functional anatomy of aortic regurgitation: accuracy, prediction of surgical repairability, and outcome implications of transesophageal echocardiography. Circulation. 2007;116: I264-269.

97. Lansac E, Di Centa I, Raoux F, Al Attar N, Acar C, Joudinaud T, Raffoul R. A lesional classification to standardize surgical management of aortic insufficiency towards valve repair. Eur J Cardiothorac Surg. 2008;33:872-878; discussion 878-880.

98. Lancellotti P, Tribouilloy C, Hagendorff A, Moura L, Popescu BA, Agricola E, Monin JL, Pierard LA, Badano L, Zamorano JL, European Association of Echocardiography. European Association of Echocardiography recommendations for the assessment of valvular regurgitation. Part 1: aortic and pulmonary regurgitation (native valve disease). Eur J Echocardiogr. 2010;11:223-244.

99. Alashi A, Khullar T, Mentias A, Gillinov AM, Roselli EE, Svensson LG, Popovic ZB, Griffin BP, Desai MY. Long-term outcomes after aortic valve surgery in patients with asymptomatic chronic aortic regurgitation and preserved LVEF: impact of baseline and follow-up global longitudinal strain. JACC Cardiovasc Imaging. 2020; 13:12-21.

100. Goldstein SA, Evangelista A, Abbara S, Arai A, Asch FM, Badano LP, Bolen MA Connolly HM, Cuellar-Calabria H, Czerny M, Devereux RB, Erbel RA, Fattori R, Isselbacher EM, Lindsay JM, McCulloch M, Michelena HI, Nienaber CA, Oh JK, Pepi M, Taylor AJ, Weinsaft JW, Zamorano JL, Dietz H, Eagle K, Elefteriades J, Jondeau G, Rousseau H, Schepens M. Multimodality imaging of diseases of the thoracic aorta in adults: from the American Society of Echocardiography and the European Association of Cardiovascular Imaging: endorsed by the Society of Cardiovascular Computed Tomography and Society for Cardiovascular Magnetic Resonance. $\mathrm{J} \mathrm{Am}$ Soc Echocardiogr. 2015;28:119-182.

101. Erbel R, Aboyans V, Boileau C, Bossone E, Bartolomeo RD, Eggebrecht H, Evangelista A, Falk V, Frank H, Gaemperli O, Grabenwoger M, Haverich A, Iung B, Manolis AJ, Meijboom F, Nienaber CA, Roffi M, Rousseau H, Sechtem U, Sirnes PA, Allmen RS, Vrints CJ, ESC Committee for Practice Guidelines. 2014 ESC Guidelines on the diagnosis and treatment of aortic diseases: document covering acute and chronic aortic diseases of the thoracic and abdominal aorta of the adult. The Task Force for the Diagnosis and Treatment of Aortic Diseases of the European Society of Cardiology (ESC ). Eur Heart J. 2014;35:2873-2926.

102. Lang RM, Badano LP, Mor-Avi V, Afilalo J, Armstrong A, Ernande L, Flachskampf FA, Foster E, Goldstein SA, Kuznetsova T, Lancellotti P, Muraru D, Picard MH, Rietzschel ER, Rudski L, Spencer KT, Tsang W, Voigt JU. Recommendations for cardiac chamber quantification by echocardiography in adults: an update from the American Society of Echocardiography and the European Association of Cardiovascular Imaging. Eur Heart J Cardiovasc Imaging. 2015;16: 233-270

103. Freeman LA, Young PM, Foley TA, Williamson EE, Bruce CJ, Greason KL. CT and MRI assessment of the aortic root and ascending aorta. AJR Am J Roentgenol. 2013;200: W581-592.

104. Amsallem M, Ou P, Milleron O, Henry-Feugeas MC, Detaint D, Arnoult F, Vahanian A, Jondeau G. Comparative assessment of ascending aortic aneurysms in Marfan patients using ECG-gated computerized tomographic angiography versus trans-thoracic echocardiography. Int J Cardiol. 2015;184:22-27.

105. Chaliki HP, Mohty D, Avierinos JF, Scott CG, Schaff HV, Tajik AJ, EnriquezSarano M. Outcomes after aortic valve replacement in patients with severe aortic regurgitation and markedly reduced left ventricular function. Circulation. 2002;106:2687-2693.

106. Kaneko T, Ejiofor JI, Neely RC, McGurk S, Ivkovic V, Stevenson LW, Leacche M, Cohn LH. Aortic regurgitation with markedly reduced left ventricular function is not a contraindication for aortic valve replacement. Ann Thorac Surg. 2016;102:41-47.

107. Tornos P, Sambola A, Permanyer-Miralda G, Evangelista A, Gomez Z, SolerSoler J. Long-term outcome of surgically treated aortic regurgitation: influence of guideline adherence toward early surgery. J Am Coll Cardiol. 2006;47:1012-1017.

108. Dujardin KS, Enriquez-Sarano M, Schaff HV, Bailey KR, Seward JB, Tajik AJ. Mortality and morbidity of aortic regurgitation in clinical practice. A long-term followup study. Circulation. 1999;99:1851-1857.

109. Klodas E, Enriquez-Sarano M, Tajik AJ, Mullany CJ, Bailey KR, Seward JB. Optimizing timing of surgical correction in patients with severe aortic regurgitation: role of symptoms. J Am Coll Cardiol. 1997;30:746-752.

110. Tribouilloy CM, Enriquez-Sarano M, Schaff HV, Orszulak TA, Fett SL, Bailey KR, Tajik AJ, Frye RL. Excess mortality due to coronary artery disease after 
valve surgery. Secular trends in valvular regurgitation and effect of internal mammary artery bypass. Circulation. 1998;98: II108-115.

111. Fiedler AG, Bhambhani V, Laikhter E, Picard MH, Wasfy MM, Tolis G, Melnitchouk S, Sundt TM, Wasfy JH. Aortic valve replacement associated with survival in severe regurgitation and low ejection fraction. Heart. 2018;104:835-840.

112. Forman R, Firth BG, Barnard MS. Prognostic significance of preoperative left ventricular ejection fraction and valve lesion in patients with aortic valve replacement. Am J Cardiol. 1980;45:1120-1125.

113. Bonow RO, Lakatos E, Maron BJ, Epstein SE. Serial long-term assessment of the natural history of asymptomatic patients with chronic aortic regurgitation and normal left ventricular systolic function. Circulation. 1991;84:1625-1635.

114. Bhudia SK, McCarthy PM, Kumpati GS, Helou J, Hoercher KJ, Rajeswaran J, Blackstone EH. Improved outcomes after aortic valve surgery for chronic aortic regurgitation with severe left ventricular dysfunction. J Am Coll Cardiol. 2007;49: 1465-1471.

115. Sambola A, Tornos P, Ferreira-Gonzalez I, Evangelista A. Prognostic value of preoperative indexed end-systolic left ventricle diameter in the outcome after surgery in patients with chronic aortic regurgitation. Am Heart J. 2008;155:1114-1120.

116. Yang LT, Michelena HI, Scott CG, Enriquez-Sarano M, Pislaru SV, Schaff HV, Pellikka PA. Outcomes in chronic hemodynamically significant aortic regurgitation and limitations of current guidelines. J Am Coll Cardiol. 2019;73:1741-1752.

117. Mentias A, Feng K, Alashi A, Rodriguez LL, Gillinov AM, Johnston DR, Sabik JF, Svensson LG, Grimm RA, Griffin BP, Desai MY. Long-term outcomes in patients with aortic regurgitation and preserved left ventricular ejection fraction. $\mathrm{J} \mathrm{Am} \mathrm{Coll} \mathrm{Cardiol.}$ 2016;68:2144-2153

118. de Meester C, Gerber BL, Vancraeynest D, Pouleur AC, Noirhomme P, Pasquet A, de Kerchove L, El Khoury G, Vanoverschelde JL. Do guideline-based indications result in an outcome penalty for patients with severe aortic regurgitation? JACC Cardiovasc Imaging. 2019;12:2126-2138.

119. Sawaya FJ, Deutsch MA, Seiffert M, Yoon SH, Codner P, Wickramarachchi U, Latib A, Petronio AS, Rodes-Cabau J, Taramasso M, Spaziano M, Bosmans J, Biasco L, Mylotte D, Savontaus M, Gheeraert P, Chan J, Jorgensen TH, Sievert H, Mocetti M, Lefevre T, Maisano F, Mangieri A, Hildick-Smith D, Kornowski R, Makkar R, Bleiziffer S, Sondergaard L, De Backer O. Safety and efficacy of transcatheter aortic valve replacement in the treatment of pure aortic regurgitation in native valves and failing surgical bioprostheses: results from an International Registry Study. JACC Cardiovasc Interv. 2017;10:1048-1056.

120. Yoon SH, Schmidt T, Bleiziffer S, Schofer N, Fiorina C, Munoz-Garcia AJ, Yzeiraj E, Amat-Santos IJ, Tchetche D, Jung C, Fujita B, Mangieri A, Deutsch MA, Ubben T, Deuschl F, Kuwata S, De Biase C, Williams T, Dhoble A, Kim WK, Ferrari E, Barbanti M, Vollema EM, Miceli A, Giannini C, Attizzani GF, Kong WKF, GutierrezIbanes E, Jimenez Diaz VA, Wijeysundera HC, Kaneko H, Chakravarty T, Makar M, Sievert H, Hengstenberg C, Prendergast BD, Vincent F, Abdel-Wahab M, NombelaFranco L, Silaschi M, Tarantini G, Butter C, Ensminger SM, Hildick-Smith D, Petronio AS, Yin WH, De Marco F, Testa L, Van Mieghem NM, Whisenant BK, Kuck KH, Colombo A, Kar S, Moris C, Delgado V, Maisano F, Nietlispach F, Mack MJ, Schofer J, Schaefer U, Bax JJ, Frerker C, Latib A, Makkar RR. Transcatheter aortic valve replacement in pure native aortic valve regurgitation. $J$ Am Coll Cardiol. 2017; 70:2752-2763.

121. Jondeau G, Detaint D, Tubach F, Arnoult F, Milleron O, Raoux F, Delorme G, Mimoun L, Krapf L, Hamroun D, Beroud C, Roy C, Vahanian A, Boileau C. Aortic event rate in the Marfan population: a cohort study. Circulation. 2012;125:226-232.

122. Desai MY, Kalahasti V, Hutt Centeno E, Chen K, Alashi A, Rivas CG, Roselli EE, Johnston DR, Griffin BP, Svensson LG. Adult patients with Marfan syndrome and ascending aortic surgery. J Am Coll Cardiol. 2019;73:733-734.

123. Borger MA, Preston M, Ivanov J, Fedak PW, Davierwala P, Armstrong S, David TE. Should the ascending aorta be replaced more frequently in patients with bicuspid aortic valve disease? J Thorac Cardiovasc Surg. 2004;128:677-683.

124. Coady MA, Rizzo JA, Hammond GL, Mandapati D, Darr U, Kopf GS, Elefteriades JA. What is the appropriate size criterion for resection of thoracic aortic aneurysms? J Thorac Cardiovasc Surg. 1997;113:476-491.

125. Davies RR, Goldstein LJ, Coady MA, Tittle SL, Rizzo JA, Kopf GS, Elefteriades JA. Yearly rupture or dissection rates for thoracic aortic aneurysms: simple prediction based on size. Ann Thorac Surg. 2002;73:17-27.

126. Oliver JM, Alonso-Gonzalez R, Gonzalez AE, Gallego P, Sanchez-Recalde A, Cuesta E, Aroca A, Lopez-Sendon JL. Risk of aortic root or ascending aorta complications in patients with bicuspid aortic valve with and without coarctation of the aorta. Am J Cardiol. 2009;104:1001-1006.

127. Michelena HI, Khanna AD, Mahoney D, Margaryan E, Topilsky Y, Suri RM, Eidem B, Edwards WD, Sundt TM 3rd, Enriquez-Sarano M. Incidence of aortic complications in patients with bicuspid aortic valves. JAMA. 2011;306:1104-1112.
128. Tzemos N, Therrien J, Yip J, Thanassoulis G, Tremblay S, Jamorski MT, Webb GD, Siu SC. Outcomes in adults with bicuspid aortic valves. JAMA. 2008;300: 1317-1325.

129. Elefteriades JA. Natural history of thoracic aortic aneurysms: indications for surgery, and surgical versus nonsurgical risks. Ann Thorac Surg. 2002;74: S1877-1880.

130. Jondeau G, Ropers J, Regalado E, Braverman A, Evangelista A, Teixedo G, De Backer J, Muino-Mosquera L, Naudion S, Zordan C, Morisaki T, Morisaki H, Von Kodolitsch Y, Dupuis-Girod S, Morris SA, Jeremy R, Odent S, Ades LC, Bakshi M, Holman K, LeMaire S, Milleron O, Langeois M, Spentchian M, Aubart M, Boileau C, Pyeritz R, Milewicz DM, Montalcino Aortic Consortium. International Registry of patients carrying TGFBR1 or TGFBR2 mutations: results of the MAC (Montalcino Aortic Consortium ). Circ Cardiovasc Genet. 2016;9:548-558.

131. Aicher D, Fries R, Rodionycheva S, Schmidt K, Langer F, Schafers HJ. Aortic valve repair leads to a low incidence of valve-related complications. Eur J Cardiothorac Surg. 2010;37:127-132.

132. de Meester C, Pasquet A, Gerber BL, Vancraeynest D, Noirhomme P, E Khoury G, Vanoverschelde JL. Valve repair improves the outcome of surgery for chronic severe aortic regurgitation: a propensity score analysis. J Thorac Cardiovasc Surg. 2014;148:1913-1920

133. Klotz S, Stock S, Sievers HH, Diwoky M, Petersen M, Stierle U, Richardt D. Survival and reoperation pattern after 20 years of experience with aortic valve-sparing root replacement in patients with tricuspid and bicuspid valves. $J$ Thorac Cardiovasc Surg. 2018;155:1403-1411 e1401

134. Elbatarny M, Tam DY, Edelman JJ, Rocha RV, Chu MWA, Peterson MD, El-Hamamsy I, Appoo JJ, Friedrich JO, Boodhwani M, Yanagawa B, Ouzounian M, Canadian Thoracic Aortic Collaborative Investigators. Valve-sparing root replacement versus composite valve grafting in aortic root dilation: a meta-analysis. Ann Thorac Surg. 2020;110:296-306.

135. Leontyev S, Schamberger L, Davierwala PM, Von Aspern K, Etz C, Lehmann S, Misfeld M, Borger MA. Early and late results after David vs Bentall procedure: a propensity matched analysis. Ann Thorac Surg. 2020;110:120-126.

136. Mastrobuoni S, de Kerchove L, Navarra E, Watremez C, Vancraeynest D, Rubay J, Noirhomme P, El Khoury G. Long-term experience with valve-sparing reimplantation technique for the treatment of aortic aneurysm and aortic regurgitation. $J$ Thorac Cardiovasc Surg. 2019;158:14-23.

137. Lansac E, Di Centa I, Sleilaty G, Lejeune S, Khelil N, Berrebi A, Diakov C, Mankoubi L, Malergue MC, Noghin M, Zannis K, Salvi S, Dervanian P, Debauchez M. Long-term results of external aortic ring annuloplasty for aortic valve repair. Eur $J$ Cardiothorac Surg. 2016;50:350-360.

138. Mazine A, Rocha RV, El-Hamamsy I, Ouzounian M, Yanagawa B, Bhatt DL, Verma S, Friedrich JO. Ross procedure vs mechanical aortic valve replacement in adults: a systematic review and meta-analysis. JAMA Cardiol. 2018;3:978-987.

139. Takkenberg JJ, Klieverik LM, Schoof PH, van Suylen RJ, van Herwerden LA, Zondervan PE, Roos-Hesselink JW, Eijkemans MJ, Yacoub MH, Bogers AJ. The Ross procedure: a systematic review and meta-analysis. Circulation. 2009;119:222-228.

140. Lee H, Cho YH, Sung K, Kim WS, Park KH, Jeong DS, Park PW, Lee YT. Clinical outcomes of root reimplantation and Bentall procedure: propensity score matching analysis. Ann Thorac Surg. 2018;106:539-547.

141. Elder DH, Wei L, Szwejkowski BR, Libianto R, Nadir A, Pauriah M, Rekhraj S, Lim TK, George J, Doney A, Pringle SD, Choy AM, Struthers AD, Lang CC. The impact of renin-angiotensin-aldosterone system blockade on heart failure outcomes and mortality in patients identified to have aortic regurgitation: a large population cohort study. J Am Coll Cardiol. 2011;58:2084-2091.

142. Seferovic PM, Ponikowski P, Anker SD, Bauersachs J, Chioncel O, Cleland JGF, de Boer RA, Drexel H, Ben Gal T, Hill L, Jaarsma T, Jankowska EA, Anker MS, Lainscak M, Lewis BS, McDonagh T, Metra M, Milicic D, Mullens W, Piepoli MF, Rosano G, Ruschitzka F, Volterrani M, Voors AA, Filippatos G, Coats AJS. Clinical practice update on heart failure 2019: pharmacotherapy, procedures, devices and patient management. An expert consensus meeting report of the Heart Failure Association of the European Society of Cardiology. Eur J Heart Fail. 2019;21: 1169-1186.

143. Lacro RV, Dietz HC, Sleeper LA, Yetman AT, Bradley TJ, Colan SD, Pearson GD, Selamet Tierney ES, Levine JC, Atz AM, Benson DW, Braverman AC, Chen S, De Backer J, Gelb BD, Grossfeld PD, Klein GL, Lai WW, Liou A, Loeys BL, Markham LW, Olson AK, Paridon SM, Pemberton VL, Pierpont ME, Pyeritz RE, Radojewski E, Roman MJ, Sharkey AM, Stylianou MP, Wechsler SB, Young LT, Mahony L, Pediatric Heart Network Investigators. Atenolol versus losartan in children and young adults with Marfan's syndrome. N Engl J Med. 2014;371:2061-2071

144. Forteza A, Evangelista A, Sanchez V, Teixido-Tura G, Sanz P, Gutierrez L, Gracia T, Centeno J, Rodriguez-Palomares J, Rufilanchas JJ, Cortina J, FerreiraGonzalez I, Garcia-Dorado D. Efficacy of losartan vs. atenolol for the prevention of aortic dilation in Marfan syndrome: a randomized clinical trial. Eur Heart J. 2016; 37:978-985 
145. Brooke BS, Habashi JP, Judge DP, Patel N, Loeys B, Dietz HC 3rd. Angiotensin II blockade and aortic-root dilation in Marfan's syndrome. $N$ Engl J Med. 2008;358: 2787-2795

146. Milleron O, Arnoult F, Ropers J, Aegerter P, Detaint D, Delorme G, Attias D, Tubach F, Dupuis-Girod S, Plauchu H, Barthelet M, Sassolas F, Pangaud N, Naudion S, Thomas-Chabaneix J, Dulac Y, Edouard T, Wolf JE, Faivre L, Odent S, Basquin A, Habib G, Collignon P, Boileau C, Jondeau G Marfan Sartan: a randomized, doubleblind, placebo-controlled trial. Eur Heart J. 2015;36:2160-2166.

147. Groenink M, den Hartog AW, Franken R, Radonic T, de Waard V, Timmermans J, Scholte AJ, van den Berg MP, Spijkerboer AM, Marquering HA, Zwinderman AH, Mulder BJ. Losartan reduces aortic dilatation rate in adults with Marfan syndrome: a randomized controlled trial. Eur Heart J. 2013;34:3491-3500.

148. Mullen M, Jin XY, Child A, Stuart AG, Dodd M, Aragon-Martin JA, Gaze D, Kiotsekoglou A, Yuan L, Hu J, Foley C, Van Dyck L, Knight R, Clayton T, Swan L, Thomson JDR, Erdem G, Crossman D, Flather M, AIMS Investigators. Irbesartan in Marfan syndrome (AIMS): a double-blind, placebo-controlled randomised trial. Lancet. 2020;394:2263-2270.

149. Pizarro R, Bazzino OO, Oberti PF, Falconi ML, Arias AM, Krauss JG, Cagide AM. Prospective validation of the prognostic usefulness of B-type natriuretic peptide in asymptomatic patients with chronic severe aortic regurgitation. $J$ Am Coll Cardiol. 2011;58:1705-1714

150. Weisenberg D, Omelchenko A, Shapira Y, Vaturi M, Monakier D, Bental T, Sagie A. Mid-term echocardiographic progression of patients with moderate aortic regurgitation: implications for aortic valve surgery. J Heart Valve Dis. 2013;22:192-194.

151. Budts W, Pieles GE, Roos-Hesselink JW, Sanz de la Garza M, D’Ascenzi F, Giannakoulas G, Muller J, Oberhoffer R, Ehringer-Schetitska D, Herceg-Cavrak V, Gabriel H, Corrado D, van Buuren F, Niebauer J, Borjesson M, Caselli S, Fritsch P, Pelliccia A, Heidbuchel H, Sharma S, Stuart AG, Papadakis M. Recommendations for participation in competitive sport in adolescent and adult athletes with Congenital Heart Disease (CHD): position statement of the Sports Cardiology \& Exercise Section of the European Association of Preventive Cardiology (EAPC), the European Society of Cardiology (ESC) Working Group on Adult Congenital Heart Disease and the Sports Cardiology, Physical Activity and Prevention Working Group of the Association for European Paediatric and Congenital Cardiology (AEPC ). Eur Heart J. 2020;41: 4191-4199.

152. d'Arcy JL, Coffey S, Loudon MA, Kennedy A, Pearson-Stuttard J, Birks J, Frangou E, Farmer AJ, Mant D, Wilson J, Myerson SG, Prendergast BD. Large-scale community echocardiographic screening reveals a major burden of undiagnosed valvular heart disease in older people: the OxVALVE Population Cohort Study. Eur Heart J 2016;37:3515-3522

153. Prihadi EA, Vollema EM, Ng ACT, Ajmone Marsan N, Bax JJ, Delgado V. Determinants and prognostic implications of left ventricular mechanical dispersion in aortic stenosis. Eur Heart J Cardiovasc Imaging. 2019;20:740-748.

154. Ilardi F, Marchetta S, Martinez C, Sprynger M, Ancion A, Manganaro R, Sugimoto T, Tsugu T, Postolache A, Piette C, Cicenia M, Esposito G, Galderisi M, Oury C, Dulgheru R, Lancellotti P. Impact of aortic stenosis on layer-specific longitudinal strain: relationship with symptoms and outcome. Eur Heart $J$ Cardiovasc Imaging. 2020;21:408-416.

155. Rusinaru D, Bohbot Y, Djelaili F, Delpierre Q, Altes A, Serbout S, Kubala M, Marechaux S, Tribouilloy C. Normative reference values of cardiac output by pulsedwave Doppler echocardiography in adults. Am J Cardiol. 2021;140:128-133.

156. Rusinaru D, Malaquin D, Marechaux S, Debry N, Tribouilloy C. Relation of dimensionless index to long-term outcome in aortic stenosis with preserved LVEF. JACC Cardiovasc Imaging. 2015;8:766-775.

157. Hachicha Z, Dumesnil JG, Bogaty P, Pibarot P. Paradoxical low-flow, low-gradient severe aortic stenosis despite preserved ejection fraction is associated with higher afterload and reduced survival. Circulation. 2007;115:2856-2864.

158. Rusinaru D, Bohbot Y, Ringle A, Marechaux S, Diouf M, Tribouilloy C. Impact of low stroke volume on mortality in patients with severe aortic stenosis and preserved left ventricular ejection fraction. Eur Heart J. 2018;39:1992-1999.

159. Guzzetti E, Poulin A, Annabi MS, Zhang B, Kalavrouziotis D, Couture C, Dagenais F, Pibarot P, Clavel MA. Transvalvular flow, sex, and survival after valve replacement surgery in patients with severe aortic stenosis. J Am Coll Cardiol. 2020;75: 1897-1909.

160. Annabi MS, Touboul E, Dahou A, Burwash IG, Bergler-Klein J, EnriquezSarano M, Orwat S, Baumgartner H, Mascherbauer J, Mundigler G, Cavalcante JL, Larose E, Pibarot P, Clavel MA. Dobutamine stress echocardiography for management of low-flow, low-gradient aortic stenosis. J Am Coll Cardiol. 2018;71:475-485.

161. Ribeiro HB, Lerakis S, Gilard M, Cavalcante JL, Makkar R, Herrmann HC, Windecker S, Enriquez-Sarano M, Cheema AN, Nombela-Franco L, Amat-Santos I, Munoz-Garcia AJ, Garcia Del Blanco B, Zajarias A, Lisko JC, Hayek S, Babaliaros V, Le Ven F, Gleason TG, Chakravarty T, Szeto WY, Clavel MA, de Agustin A, Serra V, Schindler JT, Dahou A, Puri R, Pelletier-Beaumont E, Cote M, Pibarot P, Rodes-Cabau J.
Transcatheter aortic valve replacement in patients with low-flow, low-gradient aortic stenosis: the TOPAS-TAVI Registry. J Am Coll Cardiol. 2018;71:1297-1308.

162. Clavel MA, Dumesnil JG, Capoulade R, Mathieu P, Senechal M, Pibarot P. Outcome of patients with aortic stenosis, small valve area, and low-flow, low-gradient despite preserved left ventricular ejection fraction. J Am Coll Cardiol. 2012;60: 1259-1267.

163. Clavel MA, Pibarot P, Messika-Zeitoun D, Capoulade R, Malouf J, Aggarval S, Araoz PA, Michelena HI, Cueff C, Larose E, Miller JD, Vahanian A, EnriquezSarano M. Impact of aortic valve calcification, as measured by MDCT, on survival in patients with aortic stenosis: results of an international registry study. $\mathrm{J} \mathrm{Am} \mathrm{Coll}$ Cardiol. 2014;64:1202-1213.

164. Pawade T, Clavel MA, Tribouilloy C, Dreyfus J, Mathieu T, Tastet L, Renard C, Gun M, Jenkins WSA, Macron L, Sechrist JW, Lacomis JM, Nguyen V, Galian Gay L, Cuellar Calabria H, Ntalas I, Cartlidge TRG, Prendergast B, Rajani R, Evangelista A, Cavalcante JL, Newby DE, Pibarot P, Messika Zeitoun D, Dweck MR. Computed tomography aortic valve calcium scoring in patients with aortic stenosis. Circ Cardiovasc Imaging. 2018;11: e007146.

165. Mehrotra P, Jansen K, Flynn AW, Tan TC, Elmariah S, Picard MH, Hung J. Differential left ventricular remodelling and longitudinal function distinguishes low flow from normal-flow preserved ejection fraction low-gradient severe aortic stenosis. Eur Heart J. 2013;34:1906-1914.

166. Tribouilloy C, Rusinaru D, Marechaux S, Castel AL, Debry N, Maizel J, Mentaverri R, Kamel S, Slama M, Levy F. Low-gradient, low-flow severe aortic stenosis with preserved left ventricular ejection fraction: characteristics, outcome, and implications for surgery. J Am Coll Cardiol. 2015;65:55-66.

167. Jander N, Minners J, Holme I, Gerdts E, Boman K, Brudi P, Chambers JB, Egstrup K, Kesaniemi YA, Malbecq W, Nienaber CA, Ray S, Rossebo A, Pedersen TR, Skjaerpe T, Willenheimer R, Wachtell K, Neumann FJ, Gohlke-Barwolf C. Outcome of patients with low-gradient "severe" aortic stenosis and preserved ejection fraction. Circulation. 2011;123:887-895.

168. Vollema EM, Sugimoto T, Shen M, Tastet L, Ng ACT, Abou R, Marsan NA, Mertens B, Dulgheru R, Lancellotti P, Clavel MA, Pibarot P, Genereux P, Leon MB, Delgado V, Bax JJ. Association of left ventricular global longitudinal strain with asymptomatic severe aortic stenosis: natural course and prognostic value. JAMA Cardiol. 2018;3:839-847.

169. Zamorano JL, Badano LP, Bruce C, Chan KL, Goncalves A, Hahn RT, Keane MG, La Canna G, Monaghan MJ, Nihoyannopoulos P, Silvestry FE, Vanoverschelde JL, Gillam LD. EAE/ASE recommendations for the use of echocardiography in new transcatheter interventions for valvular heart disease. Eur Heart J. 2011;32:2189-2214.

170. Bergler-Klein J, Klaar U, Heger M, Rosenhek R, Mundigler G, Gabriel H, Binder T, Pacher R, Maurer G, Baumgartner H. Natriuretic peptides predict symptomfree survival and postoperative outcome in severe aortic stenosis. Circulation. 2004;109:2302-2308.

171. Clavel MA, Malouf J, Michelena HI, Suri RM, Jaffe AS, Mahoney DW, EnriquezSarano M. B-type natriuretic peptide clinical activation in aortic stenosis: impact on long-term survival. J Am Coll Cardiol. 2014;63:2016-2025.

172. Rafique AM, Biner S, Ray I, Forrester JS, Tolstrup K, Siegel RJ. Meta-analysis of prognostic value of stress testing in patients with asymptomatic severe aortic stenosis. Am J Cardiol. 2009;104:972-977.

173. Marechaux S, Hachicha Z, Bellouin A, Dumesnil JG, Meimoun P, Pasquet A Bergeron S, Arsenault M, Le Tourneau T, Ennezat PV, Pibarot P. Usefulness of exercise-stress echocardiography for risk stratification of true asymptomatic patients with aortic valve stenosis. Eur Heart J. 2010;31:1390-1397.

174. Pawade T, Sheth T, Guzzetti E, Dweck MR, Clavel MA. Why and how to measure aortic valve calcification in patients with aortic stenosis. JACC Cardiovasc Imaging. 2019; $12: 1835-1848$.

175. Nitsche C, Scully PR, Patel KP, Kammerlander AA, Koschutnik M, Dona C, Wollenweber T, Ahmed N, Thornton GD, Kelion AD, Sabharwal N, Newton JD, Ozkor M, Kennon S, Mullen M, Lloyd G, Fontana M, Hawkins PN, Pugliese F, Menezes LJ, Moon JC, Mascherbauer J, Treibel TA. Prevalence and outcomes of concomitant aortic stenosis and cardiac amyloidosis. J Am Coll Cardiol. 2021;77: 128-139.

176. Treibel TA, Lopez B, Gonzalez A, Menacho K, Schofield RS, Ravassa S, Fontana M, White SK, DiSalvo C, Roberts N, Ashworth MT, Diez J, Moon JC. Reappraising myocardial fibrosis in severe aortic stenosis: an invasive and non-invasive study in 133 patients. Eur Heart J. 2018;39:699-709.

177. Everett RJ, Tastet L, Clavel MA, Chin CWL, Capoulade R, Vassiliou VS, Kwiecinski J, Gomez M, van Beek EJR, White AC, Prasad SK, Larose E, Tuck C, Semple S, Newby DE, Pibarot P, Dweck MR. Progression of hypertrophy and myocardial fibrosis in aortic stenosis: a multicenter cardiac magnetic resonance study. Circ Cardiovasc Imaging. 2018;11: e007451.

178. Musa TA, Treibel TA, Vassiliou VS, Captur G, Singh A, Chin C, Dobson LE, Pica S, Loudon M, Malley T, Rigolli M, Foley JRJ, Bijsterveld P, Law GR, Dweck MR, 
Myerson SG, McCann GP, Prasad SK, Moon JC, Greenwood JP. Myocardial scar and mortality in severe aortic stenosis. Circulation. 2018;138:1935-1947.

179. Ternacle J, Krapf L, Mohty D, Magne J, Nguyen A, Galat A, Gallet R, Teiger E, Cote N, Clavel MA, Tournoux F, Pibarot P, Damy T. Aortic stenosis and cardiac amyloidosis: JACC Review Topic of the Week. J Am Coll Cardiol. 2019;74:2638-2651.

180. Tribouilloy C, Levy F, Rusinaru D, Gueret P, Petit-Eisenmann H, Baleynaud S, Jobic Y, Adams C, Lelong B, Pasquet A, Chauvel C, Metz D, Quere JP, Monin JL. Outcome after aortic valve replacement for low-flow/low-gradient aortic stenosis without contractile reserve on dobutamine stress echocardiography. J Am Coll Cardiol. 2009;53:1865-1873.

181. Fougeres E, Tribouilloy C, Monchi M, Petit-Eisenmann H, Baleynaud S, Pasquet A, Chauvel C, Metz D, Adams C, Rusinaru D, Gueret P, Monin JL. Outcomes of pseudo-severe aortic stenosis under conservative treatment. Eur Heart J. 2012;33: 2426-2433.

182. Levy F, Laurent M, Monin JL, Maillet JM, Pasquet A, Le Tourneau T, PetitEisenmann H, Gori M, Jobic Y, Bauer F, Chauvel C, Leguerrier A, Tribouilloy C. Aortic valve replacement for low-flow/low-gradient aortic stenosis operative risk stratification and long-term outcome: a European multicenter study. J Am Coll Cardiol. 2008;51:1466-1472.

183. Sato K, Sankaramangalam K, Kandregula K, Bullen JA, Kapadia SR, Krishnaswamy A, Mick S, Rodriguez LL, Grimm RA, Menon V, Desai MY, Svensson LG, Griffin BP, Popovic ZB. Contemporary outcomes in low-gradient aortic stenosis patients who underwent dobutamine stress echocardiography. $J$ Am Heart Assoc. 2019;8: $\mathrm{e} 011168$.

184. Maes F, Lerakis S, Barbosa Ribeiro H, Gilard M, Cavalcante JL, Makkar R, Herrmann HC, Windecker S, Enriquez-Sarano M, Cheema AN, Nombela-Franco L, Amat-Santos I, Munoz-Garcia AJ, Garcia Del Blanco B, Zajarias A, Lisko JC, Hayek S, Babaliaros V, Le Ven F, Gleason TG, Chakravarty T, Szeto W, Clavel MA, de Agustin A, Serra V, Schindler JT, Dahou A, Salah-Annabi M, Pelletier-Beaumont E, Cote M, Puri R, Pibarot P, Rodes-Cabau J. Outcomes from transcatheter aortic valve replacement in patients with low-flow, low-gradient aortic stenosis and left ventricular ejection fraction less than $30 \%$ : a substudy from the TOPAS-TAVI Registry. JAMA Cardiol. 2019;4:64-70.

185. Chadha G, Bohbot Y, Rusinaru D, Marechaux S, Tribouilloy C. Outcome of normal-flow low-gradient severe aortic stenosis with preserved left ventricular ejection fraction: a propensity-matched study. $J$ Am Heart Assoc. 2019;8: e012301.

186. Das P, Rimington H, Chambers J. Exercise testing to stratify risk in aortic stenosis. Eur Heart J. 2005;26:1309-1313.

187. Genereux P, Stone GW, O'Gara PT, Marquis-Gravel G, Redfors B, Giustino G, Pibarot P, Bax JJ, Bonow RO, Leon MB. Natural history, diagnostic approaches, and therapeutic strategies for patients with asymptomatic severe aortic stenosis. J Am Coll Cardiol. 2016;67:2263-2288.

188. Kang DH, Park SJ, Lee SA, Lee S, Kim DH, Kim HK, Yun SC, Hong GR, Song JM, Chung CH, Song JK, Lee JW, Park SW. Early surgery or conservative care for asymptomatic aortic stenosis. $N$ Engl J Med. 2020;382:111-119.

189. Rosenhek R, Binder T, Porenta G, Lang I, Christ G, Schemper M, Maurer G, Baumgartner H. Predictors of outcome in severe, asymptomatic aortic stenosis. N Engl J Med. 2000;343:611-617.

190. Rosenhek R, Zilberszac R, Schemper M, Czerny M, Mundigler G, Graf S, Bergler-Klein J, Grimm M, Gabriel H, Maurer G. Natural history of very severe aortic stenosis. Circulation. 2010;121:151-156.

191. Cioffi G, Faggiano P, Vizzardi E, Tarantini L, Cramariuc D, Gerdts E, de Simone G. Prognostic effect of inappropriately high left ventricular mass in asymptomatic severe aortic stenosis. Heart. 2011;97:301-307.

192. Rusinaru D, Bohbot Y, Kowalski C, Ringle A, Marechaux S, Tribouilloy C. Left atrial volume and mortality in patients with aortic stenosis. J Am Heart Assoc. 2017;6: e006615.

193. Dahl JS, Videbaek L, Poulsen MK, Rudbaek TR, Pellikka PA, Moller JE. Global strain in severe aortic valve stenosis: relation to clinical outcome after aortic valve replacement. Circ Cardiovasc Imaging. 2012;5:613-620.

194. Chin CW, Shah AS, McAllister DA, Joanna Cowell S, Alam S, Langrish JP, Strachan FE, Hunter AL, Maria Choy A, Lang CC, Walker S, Boon NA, Newby DE, Mills NL, Dweck MR. High-sensitivity troponin I concentrations are a marker of an advanced hypertrophic response and adverse outcomes in patients with aortic stenosis. Eur Heart J. 2014;35:2312-2321.

195. Koos R, Brandenburg V, Mahnken AH, Muhlenbruch G, Stanzel S, Gunther RW, Floege J, Jahnen-Dechent W, Kelm M, Kuhl HP. Association of fetuin-A levels with the progression of aortic valve calcification in non-dialyzed patients. Eur Heart $J$. 2009;30:2054-2061.

196. Carroll JD, Mack MJ, Vemulapalli S, Herrmann HC, Gleason TG, Hanzel G, Deeb GM, Thourani VH, Cohen DJ, Desai N, Kirtane AJ, Fitzgerald S, Michaels J, Krohn C, Masoudi FA, Brindis RG, Bavaria JE. STS-ACC TVT Registry of transcatheter aortic valve replacement. $J$ Am Coll Cardiol. 2020;76:2492-2516.
197. Leon MB, Smith CR, Mack M, Miller DC, Moses JW, Svensson LG, Tuzcu EM, Webb JG, Fontana GP, Makkar RR, Brown DL, Block PC, Guyton RA, Pichard AD, Bavaria JE, Herrmann HC, Douglas PS, Petersen JL, Akin JJ, Anderson WN, Wang D, Pocock S, PARTNER Trial Investigators. Transcatheter aortic-valve implantation for aortic stenosis in patients who cannot undergo surgery. $N$ Engl J Med. 2010;363: 1597-1607.

198. Deeb GM, Reardon MJ, Chetcuti S, Patel HJ, Grossman PM, Yakubov SJ, Kleiman NS, Coselli JS, Gleason TG, Lee JS, Hermiller JB Jr., Heiser J, Merhi W, Zorn GL 3rd, Tadros P, Robinson N, Petrossian G, Hughes GC, Harrison JK, Maini B, Mumtaz M, Conte J, Resar J, Aharonian V, Pfeffer T, Oh JK, Qiao H, Adams DH, Popma JJ, CoreValve USCI. 3-Year outcomes in high-risk patients who underwent surgical or transcatheter aortic valve replacement. $J$ Am Coll Cardiol. 2016;67: 2565-2574

199. Smith CR, Leon MB, Mack MJ, Miller DC, Moses JW, Svensson LG, Tuzcu EM, Webb JG, Fontana GP, Makkar RR, Williams M, Dewey T, Kapadia S, Babaliaros V, Thourani VH, Corso P, Pichard AD, Bavaria JE, Herrmann HC, Akin JJ, Anderson WN, Wang D, Pocock SJ, PARTNER Trial Investigators. Transcatheter versus surgical aortic-valve replacement in high-risk patients. $N$ Engl J Med. 2011;364:2187-2198.

200. Mack MJ, Leon MB, Smith CR, Miller DC, Moses JW, Tuzcu EM, Webb JG, Douglas PS, Anderson WN, Blackstone EH, Kodali SK, Makkar RR, Fontana GP, Kapadia S, Bavaria J, Hahn RT, Thourani VH, Babaliaros V, Pichard A, Herrmann HC, Brown DL, Williams M, Akin J, Davidson MJ, Svensson LG, PARTNER 1 trial Investigators. 5-year outcomes of transcatheter aortic valve replacement or surgical aortic valve replacement for high surgical risk patients with aortic stenosis (PARTNER 1): a randomised controlled trial. Lancet. 2015;385:2477-2484.

201. Adams DH, Popma JJ, Reardon MJ, Yakubov SJ, Coselli JS, Deeb GM, Gleason TG, Buchbinder M, Hermiller J Jr., Kleiman NS, Chetcuti S, Heiser J, Merhi W, Zorn G, Tadros P, Robinson N, Petrossian G, Hughes GC, Harrison JK, Conte J, Maini B, Mumtaz M, Chenoweth S, Oh JK, Investigators USCC. Transcatheter aortic-valve replacement with a self-expanding prosthesis. N Engl J Med. 2014; 370:1790-1798

202. Thyregod HG, Steinbruchel DA, Ihlemann N, Nissen H, Kjeldsen BJ, Petursson $P$, Chang Y, Franzen OW, Engstrom T, Clemmensen P, Hansen PB, Andersen LW, Olsen PS, Sondergaard L. Transcatheter versus surgical aortic valve replacement in patients with severe aortic valve stenosis: 1-year results from the All-Comers NOTION randomized clinical trial. J Am Coll Cardiol. 2015;65:2184-2194.

203. Leon MB, Smith CR, Mack MJ, Makkar RR, Svensson LG, Kodali SK, Thourani VH, Tuzcu EM, Miller DC, Herrmann HC, Doshi D, Cohen DJ, Pichard AD, Kapadia S, Dewey T, Babaliaros V, Szeto WY, Williams MR, Kereiakes D, Zajarias A, Greason KL, Whisenant BK, Hodson RW, Moses JW, Trento A, Brown DL, Fearon WF, Pibarot P, Hahn RT, Jaber WA, Anderson WN, Alu MC, Webb JG, PARTNER 2 Investigators. Transcatheter or surgical aortic-valve replacement in intermediate-risk patients. $N$ Engl J Med. 2016;374:1609-1620.

204. Thourani VH, Kodali S, Makkar RR, Herrmann HC, Williams M, Babaliaros V, Smalling R, Lim S, Malaisrie SC, Kapadia S, Szeto WY, Greason KL, Kereiakes D, Ailawadi G, Whisenant BK, Devireddy C, Leipsic J, Hahn RT, Pibarot P, Weissman NJ, Jaber WA, Cohen DJ, Suri R, Tuzcu EM, Svensson LG, Webb JG, Moses JW, Mack MJ, Miller DC, Smith CR, Alu MC, Parvataneni R, D'Agostino RB Jr., Leon MB. Transcatheter aortic valve replacement versus surgical valve replacement in intermediate-risk patients: a propensity score analysis. Lancet. 2016;387:2218-2225.

205. Reardon MJ, Van Mieghem NM, Popma JJ, Kleiman NS, Sondergaard L, Mumtaz M, Adams DH, Deeb GM, Maini B, Gada H, Chetcuti S, Gleason T, Heiser J, Lange R, Merhi W, Oh JK, Olsen PS, Piazza N, Williams M, Windecker S, Yakubov SJ, Grube E, Makkar R, Lee JS, Conte J, Vang E, Nguyen H, Chang Y, Mugglin AS, Serruys PW, Kappetein AP, SURTAVI Investigators. Surgical or transcatheter aorticvalve replacement in intermediate-risk patients. $N$ Engl J Med. 2017;376:1321-1331. 206. Makkar RR, Thourani VH, Mack MJ, Kodali SK, Kapadia S, Webb JG, Yoon SH, Trento A, Svensson LG, Herrmann HC, Szeto WY, Miller DC, Satler L, Cohen DJ, Dewey TM, Babaliaros V, Williams MR, Kereiakes DJ, Zajarias A, Greason KL, Whisenant BK, Hodson RW, Brown DL, Fearon WF, Russo MJ, Pibarot P, Hahn RT, Jaber WA, Rogers E, Xu K, Wheeler J, Alu MC, Smith CR, Leon MB, Investigators P. Five-year outcomes of transcatheter or surgical aortic-valve replacement. $N$ Engl $J$ Med. 2020;382:799-809.

207. Thyregod HGH, Ihlemann N, Jorgensen TH, Nissen H, Kjeldsen BJ, Petursson $P$, Chang Y, Franzen OW, Engstrom T, Clemmensen P, Hansen PB, Andersen LW, Steinbruchel DA, Olsen PS, Sondergaard L. Five-year clinical and echocardiographic outcomes from the Nordic Aortic Valve Intervention (NOTION) randomized clinical trial in lower surgical risk patients. Circulation. 2019;139:2714-2723.

208. Siontis GC, Praz F, Pilgrim T, Mavridis D, Verma S, Salanti G, Sondergaard L, Juni P, Windecker $\mathrm{S}$. Transcatheter aortic valve implantation vs. surgical aortic valve replacement for treatment of severe aortic stenosis: a meta-analysis of randomized trials. Eur Heart J. 2016;37:3503-3512.

209. Mack MJ, Leon MB, Thourani VH, Makkar R, Kodali SK, Russo M, Kapadia SR, Malaisrie SC, Cohen DJ, Pibarot P, Leipsic J, Hahn RT, Blanke P, Williams MR, 
McCabe JM, Brown DL, Babaliaros V, Goldman S, Szeto WY, Genereux P, Pershad A, Pocock SJ, Alu MC, Webb JG, Smith CR, PARTNER 3 Investigators. Transcatheter aortic-valve replacement with a balloon-expandable valve in low-risk patients. $N$ Engl J Med. 2019;380:1695-1705.

210. Popma JJ, Deeb GM, Yakubov SJ, Mumtaz M, Gada H, O'Hair D, Bajwa T, Heiser JC, Merhi W, Kleiman NS, Askew J, Sorajja P, Rovin J, Chetcuti SJ, Adams DH, Teirstein PS, Zorn GL 3rd, Forrest JK, Tchetche D, Resar J, Walton A, Piazza N, Ramlawi B, Robinson N, Petrossian G, Gleason TG, Oh JK, Boulware MJ, Qiao H, Mugglin AS, Reardon MJ, Evolut Low Risk Trial Investigators. Transcatheter aorticvalve replacement with a self-expanding valve in low-risk patients. $N$ Engl J Med. 2019;380:1706-1715.

211. Siontis GCM, Overtchouk P, Cahill TJ, Modine T, Prendergast B, Praz F, Pilgrim T, Petrinic T, Nikolakopoulou A, Salanti G, Sondergaard L, Verma S, Juni P, Windecker S. Transcatheter aortic valve implantation vs. surgical aortic valve replacement for treatment of symptomatic severe aortic stenosis: an updated meta-analysis. Eur Heart J. 2019;40:3143-3153.

212. Leon MB, Mack MJ, Hahn RT, Thourani VH, Makkar R, Kodali SK, Alu MC, Madhavan MV, Chau KH, Russo M, Kapadia SR, Malaisrie SC, Cohen DJ, Blanke P, Leipsic JA, Williams MR, McCabe JM, Brown DL, Babaliaros V, Goldman S, Herrmann HC, Szeto WY, Genereux P, Pershad A, Lu M, Webb JG, Smith CR, Pibarot P, PARTNER 3 Investigators. Outcomes 2 years after transcatheter aortic valve replacement in patients at low surgical risk. J Am Coll Cardiol. 2021;77:1149-1161.

213. Greason KL, Lahr BD, Stulak JM, Cha YM, Rea RF, Schaff HV, Dearani JA. Long-term mortality effect of early pacemaker implantation after surgical aortic valve replacement. Ann Thorac Surg. 2017;104:1259-1264.

214. Auffret V, Puri R, Urena M, Chamandi C, Rodriguez-Gabella T, Philippon F, Rodes-Cabau J. Conduction disturbances after transcatheter aortic valve replacement: current status and future perspectives. Circulation. 2017;136:1049-1069.

215. Nazif TM, Chen S, George I, Dizon JM, Hahn RT, Crowley A, Alu MC, Babaliaros V, Thourani VH, Herrmann HC, Smalling RW, Brown DL, Mack MJ, Kapadia S, Makkar R, Webb JG, Leon MB, Kodali SK. New-onset left bundle branch block after transcatheter aortic valve replacement is associated with adverse long-term clinical outcomes in intermediate-risk patients: an analysis from the PARTNER II trial. Eur Heart J. 2019;40:2218-2227.

216. Tam DY, Hughes A, Wijeysundera HC, Fremes SE. Cost-effectiveness of selfexpandable transcatheter aortic valves in intermediate-risk patients. Ann Thorac Surg 2018;106:676-683.

217. Baron SJ, Wang K, House JA, Magnuson EA, Reynolds MR, Makkar R, Herrmann HC, Kodali S, Thourani VH, Kapadia S, Svensson L, Mack MJ, Brown DL, Russo MJ, Smith CR, Webb J, Miller C, Leon MB, Cohen DJ. Cost-effectiveness of transcatheter versus surgical aortic valve replacement in patients with severe aortic stenosis at intermediate risk. Circulation. 2019;139:877-888.

218. Pilgrim T, Windecker S. Expansion of transcatheter aortic valve implantation: new indications and socio-economic considerations. Eur Heart J. 2018;39: 2643-2645.

219. Barbato E, Noc M, Baumbach A, Dudek D, Bunc M, Skalidis E, Banning A, Legutko J, Witt N, Pan M, Tilsted HH, NefH, Tarantini G, Kazakiewicz D, Huculeci R, Cook S, Magdy A, Desmet W, Cayla G, Vinereanu D, Voskuil M, Goktekin O, Vardas P, Timmis A, Haude M. Mapping interventional cardiology in Europe: the European Association of Percutaneous Cardiovascular Interventions (EAPCI) Atlas Project. Eur Heart J. 2020;41:2579-2588.

220. Johnston DR, Soltesz EG, Vakil N, Rajeswaran J, Roselli EE, Sabik JF 3rd, Smedira NG, Svensson LG, Lytle BW, Blackstone EH. Long-term durability of bioprosthetic aortic valves: implications from 12,569 implants. Ann Thorac Surg. 2015;99: 1239-1247.

221. Blackman DJ, Saraf S, MacCarthy PA, Myat A, Anderson SG, Malkin CJ, Cunnington MS, Somers K, Brennan P, Manoharan G, Parker J, Aldalati O, Brecker SJ, Dowling C, Hoole SP, Dorman S, Mullen M, Kennon S, Jerrum M, Chandrala P, Roberts DH, Tay J, Doshi SN, Ludman PF, Fairbairn TA, Crowe J, Levy RD, Banning AP, Ruparelia N, Spence MS, Hildick-Smith D. Long-term durability of transcatheter aortic valve prostheses. J Am Coll Cardiol. 2019;73:537-545.

222. Barbanti M, Costa G, Zappulla P, Todaro D, Picci A, Rapisarda G, Di Simone E, Sicuso R, Buccheri S, Gulino S, Pilato G, La Spina K, D'Arrigo P, Valvo R, Indelicato A, Giannazzo D, Imme S, Tamburino C, Patane M, Sgroi C, Giuffrida A, Trovato D, Monte IP, Deste W, Capranzano P, Capodanno D, Tamburino C. Incidence of long-term structural valve dysfunction and bioprosthetic valve failure after transcatheter aortic valve replacement. $J$ Am Heart Assoc. 2018;7: e008440.

223. Didier R, Eltchaninoff H, Donzeau-Gouge P, Chevreul K, Fajadet J, Leprince P, Leguerrier A, Lievre M, Prat A, Teiger E, Lefevre T, Tchetche D, Carrie D, Himbert D, Albat B, Cribier A, Sudre A, Blanchard D, Rioufol G, Collet F, Houel R, Dos Santos P, Meneveau N, Ghostine S, Manigold T, Guyon P, Cuisset T, Le Breton H, Delepine S, Favereau X, Souteyrand G, Ohlmann P, Doisy V, Lognone T, Gommeaux A, Claudel JP, Bourlon F, Bertrand B, Iung B, Gilard M. Five-year clinical outcome and valve durability after transcatheter aortic valve replacement in high-risk patients. Circulation. 2018;138:2597-2607.

224. Deutsch MA, Erlebach M, Burri M, Hapfelmeier A, Witt OG, Ziegelmueller JA, Wottke M, Ruge H, Krane M, Piazza N, Bleiziffer S, Lange R. Beyond the five-year horizon: long-term outcome of high-risk and inoperable patients undergoing TAVR with first-generation devices. EuroIntervention. 2018;14:41-49.

225. Pibarot P, Ternacle J, Jaber WA, Salaun E, Dahou A, Asch FM, Weissman NJ, Rodriguez L, Xu K, Annabi MS, Guzzetti E, Beaudoin J, Bernier M, Leipsic J, Blanke P, Clavel MA, Rogers E, Alu MC, Douglas PS, Makkar R, Miller DC, Kapadia SR, Mack MJ, Webb JG, Kodali SK, Smith CR, Herrmann HC, Thourani VH, Leon MB, Hahn RT, PARTNER 2 Investigators. Structural deterioration of transcatheter versus surgical aortic valve bioprostheses in the PARTNER-2 Trial. $J$ Am Coll Cardiol. 2020;76:1830-1843.

226. Tam DY, Vo TX, Wijeysundera HC, Dvir D, Friedrich JO, Fremes SE. Transcatheter valve-in-valve versus redo surgical aortic valve replacement for the treatment of degenerated bioprosthetic aortic valve: a systematic review and meta-analysis. Catheter Cardiovasc Interv. 2018;92:1404-1411.

227. Deharo P, Bisson A, Herbert J, Lacour T, Etienne CS, Porto A, Theron A, Collart F, Bourguignon T, Cuisset T, Fauchier L. Transcatheter valve-in-valve aortic valve replacement as an alternative to surgical re-replacement. $\mathrm{J} \mathrm{Am}$ Coll Cardiol. 2020;76:489-499

228. Gozdek M, Raffa GM, Suwalski P, Kolodziejczak M, Anisimowicz L, Kubica J, Navarese EP, Kowalewski M, SIRIO-TAVI group. Comparative performance of transcatheter aortic valve-in-valve implantation versus conventional surgical redo aortic valve replacement in patients with degenerated aortic valve bioprostheses: systematic review and meta-analysis. Eur J Cardiothorac Surg. 2018;53:495-504.

229. Landes U, Webb JG, De Backer O, Sondergaard L, Abdel-Wahab M, Crusius L, Kim WK, Hamm C, Buzzatti N, Montorfano M, Ludwig S, Schofer N, Voigtlaender L, Guerrero M, El Sabbagh A, Rodes-Cabau J, Guimaraes L, Kornowski R, Codner P, Okuno T, Pilgrim T, Fiorina C, Colombo A, Mangieri A, Eltchaninoff H, NombelaFranco L, Van Wiechen MPH, Van Mieghem NM, Tchetche D, Schoels WH, Kullmer M, Tamburino C, Sinning JM, Al-Kassou B, Perlman GY, Danenberg H, Ielasi A, Fraccaro C, Tarantini G, De Marco F, Witberg G, Redwood SR, Lisko JC, Babaliaros VC, Laine M, Nerla R, Castriota F, Finkelstein A, Loewenstein I, Eitan A, Jaffe R, Ruile P, Neumann FJ, Piazza N, Alosaimi H, Sievert H, Sievert K, Russo M, Andreas M, Bunc M, Latib A, Govdfrey R, Hildick-Smith D, Sathananthan J, Hensey M, Alkhodair A, Blanke P, Leipsic J, Wood DA, Nazif TM, Kodali S, Leon MB, Barbanti M, Repeat transcatheter aortic valve replacement for transcatheter prosthesis dysfunction. J Am Coll Cardiol. 2020;75:1882-1893.

230. Buzzatti N, Romano V, De Backer O, Soendergaard L, Rosseel L, MaurovichHorvat P, Karady J, Merkely B, Ruggeri S, Prendergast B, De Bonis M, Colombo A, Montorfano M, Latib A. Coronary access after repeated transcatheter aortic valve implantation: a glimpse into the future. JACC Cardiovasc Imaging. 2020;13:508-515. 231. Yoon SH, Bleiziffer S, De Backer O, Delgado V, Arai T, Ziegelmueller J, Barbanti M, Sharma R, Perlman GY, Khalique OK, Holy EW, Saraf S, Deuschl F, Fujita B, Ruile P, Neumann FJ, Pache G, Takahashi M, Kaneko H, Schmidt T, Ohno Y, Schofer N, Kong WKF, Tay E, Sugiyama D, Kawamori H, Maeno Y, Abramowitz Y, Chakravarty T, Nakamura M, Kuwata S, Yong G, Kao HL, Lee M, Kim HS, Modine T, Wong SC, Bedgoni F, Testa L, Teiger E, Butter C, Ensminger SM, Schaefer U, Dvir D, Blanke P, Leipsic J, Nietlispach F, Abdel-Wahab M, Chevalier B, Tamburino C, Hildick-Smith D, Whisenant BK, Park SJ, Colombo A, Latib A, Kodali SK, Bax JJ, Sondergaard L, Webb JG, Lefevre T, Leon MB, Makkar R. Outcomes in transcatheter aortic valve replacement for bicuspid versus tricuspid aortic valve stenosis. $J$ Am Coll Cardiol. 2017;69:2579-2589.

232. Halim SA, Edwards FH, Dai D, Li Z, Mack MJ, Holmes DR, Tuzcu EM, Thourani VH, Harrison JK, Brennan JM. Outcomes of transcatheter aortic valve replacement in patients with bicuspid aortic valve disease: a report from the Society of Thoracic Surgeons/American College of Cardiology Transcatheter Valve Therapy Registry. Circulation. 2020;141:1071-1079.

233. Forrest JK, Kaple RK, Ramlawi B, Gleason TG, Meduri CU, Yakubov SJ, Jilaihawi H, Liu F, Reardon MJ. Transcatheter aortic valve replacement in bicuspid versus tricuspid aortic valves from the STS/ACC TVT Registry. JACC Cardiovasc Interv. 2020;13:1749-1759.

234. Alkhouli M, Zack CJ, Sarraf M, Bashir R, Nishimura RA, Eleid MF, Nkomo VT, Sandhu GS, Gulati R, Greason KL, Holmes DR, Rihal CS. Morbidity and mortality associated with balloon aortic valvuloplasty: a national perspective. Circ Cardiovasc Interv. 2017;10.

235. Horstkotte D, Loogen F. The natural history of aortic valve stenosis. Eur Heart $J$. 1988;9 Suppl E: 57-64.

236. Lund O. Preoperative risk evaluation and stratification of long-term survival after valve replacement for aortic stenosis. Reasons for earlier operative intervention. Circulation. 1990;82:124-139.

237. Mangner N, Stachel G, Woitek F, Haussig S, Schlotter F, Hollriegel R, Adam J, Lindner A, Mohr FW, Schuler G, Kiefer P, Leontyev S, Borger MA, Thiele H, 
Holzhey D, Linke A. Predictors of mortality and symptomatic outcome of patients with low-flow severe aortic stenosis undergoing transcatheter aortic valve replacement. $J$ Am Heart Assoc. 2018;7: e007977.

238. Dahl JS, Eleid MF, Michelena HI, Scott CG, Suri RM, Schaff HV, Pellikka PA. Effect of left ventricular ejection fraction on postoperative outcome in patients with severe aortic stenosis undergoing aortic valve replacement. Circ Cardiovasc Imaging. 2015;8: e002917.

239. Taniguchi T, Morimoto T, Shiomi H, Ando K, Kanamori N, Murata K, Kitai T, Kadota K, Izumi C, Nakatsuma K, Sasa T, Watanabe H, Kuwabara Y, Makiyama T, Ono K, Shizuta S, Kato T, Saito N, Minatoya K, Kimura T, CURRENT AS Registry Investigators. Prognostic impact of left ventricular ejection fraction in patients with severe aortic stenosis. JACC Cardiovasc Interv. 2018;11:145-157.

240. Bohbot Y, de Meester de Ravenstein C, Chadha G, Rusinaru D, Belkhir K, Trouillet C, Pasquet A, Marechaux S, Vanoverschelde JL, Tribouilloy C. Relationship between left ventricular ejection fraction and mortality in asymptomatic and minimally symptomatic patients with severe aortic stenosis. JACC Cardiovasc Imaging. 2019;12: 38-48.

241. Capoulade R, Le Ven F, Clavel MA, Dumesnil JG, Dahou A, Thebault C, Arsenault M, O'Connor K, Bedard E, Beaudoin J, Senechal M, Bernier M, Pibarot P. Echocardiographic predictors of outcomes in adults with aortic stenosis. Heart. 2016;102:934-942.

242. Bohbot Y, Kowalski C, Rusinaru D, Ringle A, Marechaux S, Tribouilloy C. Impact of mean transaortic pressure gradient on long-term outcome in patients with severe aortic stenosis and preserved left ventricular ejection fraction. J Am Heart Assoc. 2017;6.

243. Otto CM, Burwash IG, Legget ME, Munt BI, Fujioka M, Healy NL, Kraft CD, Miyake-Hull CY, Schwaegler RG. Prospective study of asymptomatic valvular aortic stenosis. Clinical, echocardiographic, and exercise predictors of outcome. Circulation. 1997;95:2262-2270.

244. Thourani VH, Suri RM, Gunter RL, Sheng S, O’Brien SM, Ailawadi G, Szeto WY, Dewey TM, Guyton RA, Bavaria JE, Babaliaros V, Gammie JS, Svensson L, Williams M, Badhwar V, Mack MJ. Contemporary real-world outcomes of surgical aortic valve replacement in 141,905 low-risk, intermediate-risk, and high-risk patients. Ann Thorac Surg. 2015;99:55-61.

245. Gleason TG, Reardon MJ, Popma JJ, Deeb GM, Yakubov SJ, Lee JS, Kleiman NS, Chetcuti S, Hermiller JB Jr., Heiser J, Merhi W, Zorn GL 3rd, Tadros P, Robinson N, Petrossian G, Hughes GC, Harrison JK, Conte JV, Mumtaz M, Oh JK, Huang J, Adams DH, CoreValve US Pivotal High Risk Trial Clinical Investigators. 5-Year outcomes of self-expanding transcatheter versus surgical aortic valve replacement in highrisk patients. J Am Coll Cardiol. 2018;72:2687-2696.

246. Rossebo AB, Pedersen TR, Boman K, Brudi P, Chambers JB, Egstrup K, Gerdts E, Gohlke-Barwolf C, Holme I, Kesaniemi YA, Malbecq W, Nienaber CA, Ray S, Skjaerpe T, Wachtell K, Willenheimer R, SEAS Investigators. Intensive lipid lowering with simvastatin and ezetimibe in aortic stenosis. $N$ Engl J Med. 2008;359: 1343-1356.

247. Ponikowski P, Voors AA, Anker SD, Bueno H, Cleland JGF, Coats AJS, Falk V, Gonzalez-Juanatey JR, Harjola VP, Jankowska EA, Jessup M, Linde C, Nihoyannopoulos P, Parissis JT, Pieske B, Riley JP, Rosano GMC, Ruilope LM, Ruschitzka F, Rutten FH, van der Meer P, ESC Scientific Document Group. 2016 ESC Guidelines for the diagnosis and treatment of acute and chronic heart failure: The Task Force for the diagnosis and treatment of acute and chronic heart failure of the European Society of Cardiology (ESC) Developed with the special contribution of the Heart Failure Association (HFA) of the ESC. Eur Heart J. 2016;37:2129-2200.

248. Bull S, Loudon M, Francis JM, Joseph J, Gerry S, Karamitsos TD, Prendergast BD, Banning AP, Neubauer S, Myerson SG. A prospective, double-blind, randomized controlled trial of the angiotensin-converting enzyme inhibitor Ramipril In Aortic Stenosis (RIAS trial). Eur Heart J Cardiovasc Imaging. 2015; 16:834-841.

249. Ochiai T, Saito S, Yamanaka F, Shishido K, Tanaka Y, Yamabe T, Shirai S, Tada N, Araki M, Naganuma T, Watanabe Y, Yamamoto M, Hayashida K. Renin-angiotensin system blockade therapy after transcatheter aortic valve implantation. Heart. 2018;104:644-651.

250. Dahl JS, Videbaek L, Poulsen MK, Pellikka PA, Veien K, Andersen LI, Haghfelt T, Moller JE. Effect of candesartan treatment on left ventricular remodeling after aortic valve replacement for aortic stenosis. Am J Cardiol. 2010;106:713-719.

251. Strange G, Stewart S, Celermajer D, Prior D, Scalia GM, Marwick T, Ilton M, Joseph M, Codde J, Playford D, National Echocardiography Database of Australia contributing sites. Poor long-term survival in patients with moderate aortic stenosis. $\mathrm{J} \mathrm{Am}$ Coll Cardiol. 2019;74:1851-1863.

252. van Gils L, Clavel MA, Vollema EM, Hahn RT, Spitzer E, Delgado V, Nazif T, De Jaegere PP, Geleijnse ML, Ben-Yehuda O, Bax JJ, Leon MB, Pibarot P, Van Mieghem NM. Prognostic implications of moderate aortic stenosis in patients with left ventricular systolic dysfunction. J Am Coll Cardiol. 2017;69:2383-2392.
253. Delesalle G, Bohbot Y, Rusinaru D, Delpierre Q, Marechaux S, Tribouilloy C. Characteristics and prognosis of patients with moderate aortic stenosis and preserved left ventricular ejection fraction. J Am Heart Assoc. 2019;8: e011036.

254. Samad Z, Vora AN, Dunning A, Schulte PJ, Shaw LK, Al-Enezi F, Ersboll M, McGarrah RW 3rd, Vavalle JP, Shah SH, Kisslo J, Glower D, Harrison JK, Velazquez EJ. Aortic valve surgery and survival in patients with moderate or severe aortic stenosis and left ventricular dysfunction. Eur Heart J. 2016;37:2276-2286.

255. Chaker Z, Badhwar V, Alqahtani F, Aljohani S, Zack CJ, Holmes DR, Rihal CS, Alkhouli M. Sex differences in the utilization and outcomes of surgical aortic valve replacement for severe aortic stenosis. J Am Heart Assoc. 2017;6.

256. Cote N, Clavel MA. Sex differences in the pathophysiology, diagnosis, and management of aortic stenosis. Cardiol Clin. 2020;38:129-138.

257. Tribouilloy C, Bohbot Y, Rusinaru D, Belkhir K, Diouf M, Altes A, Delpierre Q, Serbout S, Kubala M, Levy F, Marechaux S, Enriquez Sarano M. Excess mortality and undertreatment of women with severe aortic stenosis. J Am Heart Assoc. 2021;10: e018816.

258. Smith WTt, Ferguson TB Jr., Ryan T, Landolfo CK, Peterson ED. Should coronary artery bypass graft surgery patients with mild or moderate aortic stenosis undergo concomitant aortic valve replacement? A decision analysis approach to the surgical dilemma. J Am Coll Cardiol. 2004;44:1241-1247.

259. Faroux L, Campelo-Parada F, Munoz-Garcia E, Nombela-Franco L, Fischer Q, Donaint P, Serra V, Veiga G, Gutierrez E, Vilalta V, Alperi A, Regueiro A, Asmarats L, Ribeiro HB, Matta A, Munoz-Garcia A, Armijo G, Urena M, Metz D, RodenasAlesina E, la de Torre Hernandez JM, Fernandez-Nofrerias E, Pascual I, PerezFuentes P, Arzamendi D, Campanha-Borges DC, Del Val D, Couture T, Rodes-Cabau J. Procedural characteristics and late outcomes of percutaneous coronary intervention in the workup pre-TAVR. JACC Cardiovasc Interv. 2020;13:2601-2613.

260. Sondergaard L, Popma JJ, Reardon MJ, Van Mieghem NM, Deeb GM, Kodali S, George I, Williams MR, Yakubov SJ, Kappetein AP, Serruys PW, Grube E, Schiltgen MB, Chang Y, Engstrom T, SURTAVI Trial Investigators. Comparison of a complete percutaneous versus surgical approach to aortic valve replacement and revascularization in patients at intermediate surgical risk: results from the randomized SURTAVI Trial. Circulation. 2019;140:1296-1305.

261. Nombela-Franco L, Eltchaninoff H, Zahn R, Testa L, Leon MB, Trillo-Nouche R, D'Onofrio A, Smith CR, Webb J, Bleiziffer S, De Chiara B, Gilard M, Tamburino C, Bedogni F, Barbanti M, Salizzoni S, Garcia del Blanco B, Sabate M, Moreo A, Fernandez C, Ribeiro HB, Amat-Santos I, Urena M, Allende R, Garcia E, Macaya C, Dumont E, Pibarot P, Rodes-Cabau J. Clinical impact and evolution of mitral regurgitation following transcatheter aortic valve replacement: a meta-analysis. Heart. 2015; 101:1395-1405

262. D’Ancona G, Kische S, Senges J, Ouarrak T, Puls M, Bekeredjian R, Sievert H, Safak E, Ortak J, Oner A, Schillinger W, Ince H. Combined mitro-aortic pathology: impact of previous aortic valve replacement upon outcomes of MitraClip therapy (from the German transcatheter mitral valve interventions registry). EuroIntervention. 2017; $13: 475-482$

263. Witberg G, Codner P, Landes U, Barbanti M, Valvo R, De Backer O, Ooms JF, Sievert K, El Sabbagh A, Jimenez-Quevedo P, Brennan PF, Sedaghat A, Masiero G, Werner P, Overtchouk P, Watanabe Y, Montorfano M, Bijjam VR, Hein M, Fiorina C, Arzamendi D, Rodriguez-Gabella T, Fernandez-Vazquez F, Baz JA, Laperche C, Grasso C, Branca L, Estevez-Loureiro R, Benito-Gonzalez T, Amat Santos IJ, Ruile P, Mylotte D, Buzzatti N, Piazza N, Andreas M, Tarantini G, Sinning JM, Spence MS, Nombela-Franco L, Guerrero M, Sievert H, Sondergaard L, Van Mieghem NM, Tchetche D, Webb JG, Kornowski R. Transcatheter treatment of residual significan mitral regurgitation following TAVR: a multicenter registry. JACC Cardiovasc Interv. 2020;13:2782-2791

264. Khan F, Okuno T, Malebranche D, Lanz J, Praz F, Stortecky S, Windecker S, Pilgrim T. Transcatheter aortic valve replacement in patients with multivalvular heart disease. JACC Cardiovasc Interv. 2020;13:1503-1514.

265. Baumgartner H, Bonhoeffer P, De Groot NM, de Haan F, Deanfield JE, Galie N, Gatzoulis MA, Gohlke-Baerwolf C, Kaemmerer H, Kilner P, Meijboom F, Mulder BJ, Oechslin E, Oliver JM, Serraf A, Szatmari A, Thaulow E, Vouhe PR, Walma E, Task Force on the Management of Grown-up Congenital Heart Disease of the European Society of Cardiology, Association for European Paediatric Cardiology, ESC Committee for Practice Guidelines. ESC Guidelines for the management of grown-up congenital heart disease (new version 2010 ). Eur Heart J. 2010;31:2915-2957.

266. Dziadzko V, Dziadzko M, Medina-Inojosa JR, Benfari G, Michelena HI, Crestanello JA, Maalouf J, Thapa P, Enriquez-Sarano M. Causes and mechanisms of isolated mitral regurgitation in the community: clinical context and outcome. Eur Heart J. 2019;40:2194-2202.

267. Kingue S, Ba SA, Balde D, Diarra MB, Anzouan-Kacou JB, Anisubia B, Damorou JM, Ndobo P, Menanga A, Kane A, Kakou-Guikahue M, Kenfack M, Metogo B, Chelo D, Yangnigni E, Tantchou C, Bertrand E, Monsuez JJ, Working Group on Tropical Cardiology of the Société française de cardiologie. The VALVAFRIC 
study: a registry of rheumatic heart disease in Western and Central Africa. Arch Cardiovasc Dis. 2016;109:321-329.

268. Zoghbi WA, Adams D, Bonow RO, Enriquez-Sarano M, Foster E, Grayburn PA, Hahn RT, Han Y, Hung J, Lang RM, Little SH, Shah DJ, Shernan S, Thavendiranathan P, Thomas JD, Weissman NJ. Recommendations for noninvasive evaluation of native valvular regurgitation: a report from the American Society of Echocardiography developed in collaboration with the Society for Cardiovascular Magnetic Resonance. J Am Soc Echocardiogr. 2017;30:303-371.

269. Antoine C, Benfari G, Michelena HI, Maalouf JF, Nkomo VT, Thapa P, EnriquezSarano M. Clinical outcome of degenerative mitral regurgitation. Circulation. 2018;138:1317-1326.

270. Samad Z, Shaw LK, Phelan M, Glower DD, Ersboll M, Toptine JH, Alexander JH, Kisslo JA, Wang A, Mark DB, Velazquez EJ. Long-term outcomes of mitral regurgitation by type and severity. Am Heart J. 2018;203:39-48.

271. Carpentier A. Cardiac valve surgery-the "French correction ". J Thorac Cardiovasc Surg. 1983;86:323-337.

272. Boekstegers P, Hausleiter J, Baldus S, von Bardeleben RS, Beucher H, Butter C, Franzen O, Hoffmann R, Ince H, Kuck KH, Rudolph V, Schafer U, Schillinger W, Wunderlich N, Germany Society of Cardiology Working Group on Interventional Cardiology Focus Group on Interventional Mitral Valve Therapy. Percutaneous interventional mitral regurgitation treatment using the Mitra-Clip system. Clin Res Cardiol. 2014;103:85-96.

273. Gavazzoni M, Taramasso M, Zuber M, Russo G, Pozzoli A, Miura M, Maisano F. Conceiving MitraClip as a tool: percutaneous edge-to-edge repair in complex mitral valve anatomies. Eur Heart J Cardiovasc Imaging. 2020;21:1059-1067.

274. Cawley PJ, Hamilton-Craig C, Owens DS, Krieger EV, Strugnell WE, Mitsumori L, D’Jang CL, Schwaegler RG, Nguyen KQ, Nguyen B, Maki JH, Otto CM. Prospective comparison of valve regurgitation quantitation by cardiac magnetic resonance imaging and transthoracic echocardiography. Circ Cardiovasc Imaging. 2013;6:48-57.

275. Shanks M, Siebelink HM, Delgado V, van de Veire NR, Ng AC, Sieders A, Schuijf JD, Lamb HJ, Ajmone Marsan N, Westenberg JJ, Kroft LJ, de Roos A, Bax JJ. Quantitative assessment of mitral regurgitation: comparison between three-dimensional transesophageal echocardiography and magnetic resonance imaging. Circ Cardiovasc Imaging. 2010;3:694-700.

276. Uretsky S, Gillam L, Lang R, Chaudhry FA, Argulian E, Supariwala A, Gurram S, Jain K, Subero M, Jang JJ, Cohen R, Wolff SD. Discordance between echocardiography and MRI in the assessment of mitral regurgitation severity: a prospective multicenter trial. J Am Coll Cardiol. 2015;65:1078-1088.

277. Penicka M, Vecera J, Mirica DC, Kotrc M, Kockova R, Van Camp G. Prognostic implications of magnetic resonance-derived quantification in asymptomatic patients with organic mitral regurgitation: comparison with Doppler echocardiography-derived integrative approach. Circulation. 2018;137:1349-1360.

278. Garg P, Swift AJ, Zhong L, Carlhall CJ, Ebbers T, Westenberg J, Hope MD, Bucciarelli-Ducci C, Bax JJ, Myerson SG. Assessment of mitral valve regurgitation by cardiovascular magnetic resonance imaging. Nat Rev Cardiol. 2020;17:298-312.

279. Kitkungvan D, Nabi F, Kim RJ, Bonow RO, Khan MA, Xu J, Little SH, Quinones MA, Lawrie GM, Zoghbi WA, Shah DJ. Myocardial fibrosis in patients with primary mitral regurgitation with and without prolapse. J Am Coll Cardiol. 2018; 72:823-834.

280. Bakkestrom R, Banke A, Christensen NL, Pecini R, Irmukhamedov A, Andersen M, Borlaug BA, Moller JE. Hemodynamic characteristics in significant symptomatic and asymptomatic primary mitral valve regurgitation at rest and during exercise. Circ Cardiovasc Imaging. 2018;11: e007171.

281. Utsunomiya H, Hidaka T, Susawa H, Izumi K, Harada Y, Kinoshita M, Itakura K, Masada K, Kihara Y. Exercise-stress echocardiography and effort intolerance in asymptomatic/minimally symptomatic patients with degenerative mitral regurgitation combined invasive-noninvasive hemodynamic monitoring. Circ Cardiovasc Imaging. 2018;11: e007282.

282. Pizarro R, Bazzino OO, Oberti PF, Falconi M, Achilli F, Arias A, Krauss JG, Cagide AM. Prospective validation of the prognostic usefulness of brain natriuretic peptide in asymptomatic patients with chronic severe mitral regurgitation. J Am Coll Cardiol. 2009;54:1099-1106.

283. Hiemstra YL, Tomsic A, van Wijngaarden SE, Palmen M, Klautz RJM, Bax JJ, Delgado V, Ajmone Marsan N. Prognostic value of global longitudinal strain and etiology after surgery for primary mitral regurgitation. JACC Cardiovasc Imaging. 2020;13:577-585.

284. Kim HM, Cho GY, Hwang IC, Choi HM, Park JB, Yoon YE, Kim HK. Myocardial strain in prediction of outcomes after surgery for severe mitral regurgitation. JACC Cardiovasc Imaging. 2018;11:1235-1244.

285. Grigioni F, Clavel MA, Vanoverschelde JL, Tribouilloy C, Pizarro R, Huebner M, Avierinos JF, Barbieri A, Suri R, Pasquet A, Rusinaru D, Gargiulo GD, Oberti P, Theron A, Bursi F, Michelena H, Lazam S, Szymanski C, Nkomo VT, Schumacher M,
Bacchi-Reggiani L, Enriquez-Sarano M, MIDA Investigators. The MIDA Mortality Risk Score: development and external validation of a prognostic model for early and late death in degenerative mitral regurgitation. Eur Heart J. 2018;39:1281-1291.

286. Tribouilloy C, Grigioni F, Avierinos JF, Barbieri A, Rusinaru D, Szymanski C, Ferlito M, Tafanelli L, Bursi F, Trojette F, Branzi A, Habib G, Modena MG, EnriquezSarano M, MIDA Investigators. Survival implication of left ventricular end-systolic diameter in mitral regurgitation due to flail leaflets a long-term follow-up multicenter study. J Am Coll Cardiol. 2009;54:1961-1968.

287. Essayagh B, Antoine C, Benfari G, Messika-Zeitoun D, Michelena H, Le Tourneau T, Mankad S, Tribouilloy CM, Thapa P, Enriquez-Sarano M. Prognostic implications of left atrial enlargement in degenerative mitral regurgitation. $J$ Am Coll Cardiol. 2019;74:858-870.

288. Rusinaru D, Tribouilloy C, Grigioni F, Avierinos JF, Suri RM, Barbieri A, Szymanski C, Ferlito M, Michelena H, Tafanelli L, Bursi F, Mezghani S, Branzi A, Habib G, Modena MG, Enriquez-Sarano M, Mitral Regurgitation International DAtabase (MIDA) Investigators. Left atrial size is a potent predictor of mortality in mitral regurgitation due to flail leaflets: results from a large international multicenter study. Circ Cardiovasc Imaging. 2011;4:473-481.

289. Barbieri A, Bursi F, Grigioni F, Tribouilloy C, Avierinos JF, Michelena HI, Rusinaru D, Szymansky C, Russo A, Suri R, Bacchi Reggiani ML, Branzi A, Modena MG, Enriquez-Sarano M, Mitral Regurgitation International DAtabase (MIDA) Investigators. Prognostic and therapeutic implications of pulmonary hypertension complicating degenerative mitral regurgitation due to flail leaflet: a multicenter long-term international study. Eur Heart J. 2011;32:751-759.

290. Grigioni F, Benfari G, Vanoverschelde JL, Tribouilloy C, Avierinos JF, Bursi F, Suri RM, Guerra F, Pasquet A, Rusinaru D, Marcelli E, Theron A, Barbieri A, Michelena H, Lazam S, Szymanski C, Nkomo VT, Capucci A, Thapa P, EnriquezSarano M, MIDA Investigators. Long-term implications of atrial fibrillation in patients with degenerative mitral regurgitation. J Am Coll Cardiol. 2019;73:264-274.

291. Szymanski C, Magne J, Fournier A, Rusinaru D, Touati G, Tribouilloy C. Usefulness of preoperative atrial fibrillation to predict outcome and left ventricular dysfunction after valve repair for mitral valve prolapse. Am J Cardiol. 2015; 115:1448-1453.

292. Suri RM, Vanoverschelde JL, Grigioni F, Schaff HV, Tribouilloy C, Avierinos JF, Barbieri A, Pasquet A, Huebner M, Rusinaru D, Russo A, Michelena HI, EnriquezSarano M. Association between early surgical intervention vs watchful waiting and outcomes for mitral regurgitation due to flail mitral valve leaflets. JAMA. 2013; 310:609-616.

293. Jung JC, Jang MJ, Hwang HY. Meta-analysis comparing mitral valve repair versus replacement for degenerative mitral regurgitation across all ages. Am J Cardiol. 2019;123:446-453.

294. Lazam S, Vanoverschelde JL, Tribouilloy C, Grigioni F, Suri RM, Avierinos JF, de Meester C, Barbieri A, Rusinaru D, Russo A, Pasquet A, Michelena HI, Huebner M, Maalouf J, Clavel MA, Szymanski C, Enriquez-Sarano M, MIDA Investigators. Twenty-year outcome after mitral repair versus replacement for severe degenerative mitral regurgitation: analysis of a large, prospective, multicenter, international registry. Circulation. 2017; 135:410-422.

295. Chikwe J, Toyoda N, Anyanwu AC, Itagaki S, Egorova NN, Boateng P, El-Eshmawi A, Adams DH. Relation of mitral valve surgery volume to repair rate, durability, and survival. J Am Coll Cardiol 2017; doi: 10.1016/j.jacc.2017.02.026.

296. David TE, David CM, Tsang W, Lafreniere-Roula M, Manlhiot C. Long-term results of mitral valve repair for regurgitation due to leaflet prolapse. J Am Coll Cardiol. 2019;74:1044-1053.

297. Donnellan E, Alashi A, Johnston DR, Gillinov AM, Pettersson GB, Svensson LG, Griffin BP, Desai MY. Outcomes of patients with mediastinal radiation-associated mitral valve disease undergoing cardiac surgery. Circulation. 2019;140:1288-1290.

298. Vakamudi S, Jellis C, Mick S, Wu Y, Gillinov AM, Mihaljevic T, Cosgrove DM, Svensson L, Cho L. Sex differences in the etiology of surgical mitral valve disease. Circulation. 2018;138:1749-1751.

299. Feldman T, Foster E, Glower DD, Kar S, Rinaldi MJ, Fail PS, Smalling RW, Siegel R, Rose GA, Engeron E, Loghin C, Trento A, Skipper ER, Fudge T, Letsou GV, Massaro JM, Mauri L, EVEREST II Investigators. Percutaneous repair or surgery for mitral regurgitation. $N$ Engl J Med. 2011;364:1395-1406.

300. Feldman T, Kar S, Elmariah S, Smart SC, Trento A, Siegel RJ, Apruzzese P, Fail P, Rinaldi MJ, Smalling RW, Hermiller JB, Heimansohn D, Gray WA, Grayburn PA, Mack MJ, Lim DS, Ailawadi G, Herrmann HC, Acker MA, Silvestry FE, Foster E, Wang A, Glower DD, Mauri L, EVEREST II Investigators. Randomized comparison of percutaneous repair and surgery for mitral regurgitation: 5-year results of EVEREST II. J Am Coll Cardiol. 2015;66:2844-2854.

301. Buzzatti N, Van Hemelrijck M, Denti P, Ruggeri S, Schiavi D, Scarfo IS, Reser D, Taramasso M, Weber A, La Canna G, De Bonis M, Maisano F, Alfieri O. Transcatheter or surgical repair for degenerative mitral regurgitation in elderly patients: a propensityweighted analysis. J Thorac Cardiovasc Surg. 2019;158:86-94 e81. 
302. Sorajja P, Vemulapalli S, Feldman T, Mack M, Holmes DR Jr., Stebbins A, Kar S, Thourani V, Ailawadi G. Outcomes with transcatheter mitral valve repair in the United States: an STS/ACC TVT Registry Report. J Am Coll Cardiol. 2017;70:2315-2327.

303. Gammie JS, Bartus K, Gackowski A, D’Ambra MN, Szymanski P, Bilewska A, Kusmierczyk M, Kapelak B, Rzucidlo-Resil J, Moat N, Duncan A, Yadev R, Livesey S, Diprose P, Gerosa G, D'Onofrio A, Pitterello D, Denti P, La Canna G, De Bonis M, Alfieri $\mathrm{O}$, Hung J, Kolsut P. Beating-heart mitral valve repair using a novel ePTFE cordal implantation device: a prospective trial. J Am Coll Cardiol. 2018;71:25-36.

304. Lim DS, Kar S, Spargias K, Kipperman RM, O’Neill WW, Ng MKC, Fam NP, Walters DL, Webb JG, Smith RL, Rinaldi MJ, Latib A, Cohen GN, Schafer U, Marcoff L, Vandrangi P, Verta P, Feldman TE. Transcatheter valve repair for patients with mitral regurgitation: 30 -day results of the CLASP Study. JACC Cardiovasc Interv. 2019;12:1369-1378.

305. Praz F, Braun D, Unterhuber M, Spirito A, Orban M, Brugger N, Brinkmann I, Spring K, Moschovitis A, Nabauer M, Blazek S, Pilgrim T, Thiele H, Lurz P, Hausleiter J, Windecker S. Edge-to-edge mitral valve repair with extended clip arms: early experience from a multicenter observational study. JACC Cardiovasc Interv. 2019;12:1356-1365.

306. Praz F, Spargias K, Chrissoheris M, Bullesfeld L, Nickenig G, Deuschl F, Schueler R, Fam NP, Moss R, Makar M, Boone R, Edwards J, Moschovitis A, Kar S, Webb J, Schafer U, Feldman T, Windecker S. Compassionate use of the PASCAL transcatheter mitral valve repair system for patients with severe mitral regurgitation: a multicentre, prospective, observational, first-in-man study. Lancet. 2017;390:773-780.

307. Chakravarty T, Makar M, Patel D, Oakley L, Yoon SH, Stegic J, Singh S, Skaf S, Nakamura M, Makkar RR. Transcatheter edge-to-edge mitral valve repair with the MitraClip G4 system. JACC Cardiovasc Interv. 2020;13:2402-2414.

308. Piriou N, Al Habash O, Donal E, Senage T, Le Tourneau T, Pattier S, Guyomarch B, Roussel JC, Trochu JN, Vahanian A, Obadia JF, Iung B, Guerin P. The MITRA-HR study: design and rationale of a randomised study of MitraClip transcatheter mitral valve repair in patients with severe primary mitral regurgitation eligible for high-risk surgery. EuroIntervention. 2019;15: e329-e335.

309. Zilberszac R, Heinze G, Binder T, Laufer G, Gabriel H, Rosenhek R. Long-term outcome of active surveillance in severe but asymptomatic primary mitral regurgitation. JACC Cardiovasc Imaging. 2018;11:1213-1221.

310. Dejgaard LA, Skjolsvik ET, Lie OH, Ribe M, Stokke MK, Hegbom F, Scheirlynck ES, Gjertsen E, Andresen K, Helle-Valle TM, Hopp E, Edvardsen T, Haugaa KH. The mitral annulus disjunction arrhythmic syndrome. J Am Coll Cardiol. 2018;72:1600-1609.

311. Han HC, Ha FJ, Teh AW, Calafiore P, Jones EF, Johns J, Koshy AN, O’Donnell D, Hare DL, Farouque O, Lim HS. Mitral valve prolapse and sudden cardiac death: a systematic review. J Am Heart Assoc. 2018;7: e010584.

312. Nalliah CJ, Mahajan R, Elliott AD, Haqqani H, Lau DH, Vohra JK, Morton JB, Semsarian C, Marwick T, Kalman JM, Sanders P. Mitral valve prolapse and sudden cardiac death: a systematic review and meta-analysis. Heart. 2019;105:144-151.

313. Essayagh B, Sabbag A, Antoine C, Benfari G, Yang LT, Maalouf J, Asirvatham S, Michelena H, Enriquez-Sarano M. Presentation and outcome of arrhythmic mitral valve prolapse. J Am Coll Cardiol. 2020;76:637-649.

314. Lancellotti P, Pibarot P, Chambers J, Edvardsen T, Delgado V, Dulgheru R, Pepi M, Cosyns B, Dweck MR, Garbi M, Magne J, Nieman K, Rosenhek R, Bernard A, Lowenstein J, Vieira ML, Rabischoffsky A, Vyhmeister RH, Zhou X, Zhang Y, Zamorano JL, Habib G. Recommendations for the imaging assessment of prosthetic heart valves: a report from the European Association of Cardiovascular Imaging endorsed by the Chinese Society of Echocardiography, the Inter-American Society of Echocardiography, and the Brazilian Department of Cardiovascular Imaging. Eur Heart J Cardiovasc Imaging. 2016;17:589-590.

315. Yu M, Georges A, Tucker NR, Kyryachenko S, Toomer K, Schott JJ, Delling FN, Fernandez-Friera L, Solis J, Ellinor PT, Levine RA, Slaugenhaupt SA, Hagege AA, Dina C, Jeunemaitre X, Milan DJ, Norris RA, Bouatia-Naji N. Genome-wide association study-driven gene-set analyses, genetic, and functional follow-up suggest GLIS1 as a susceptibility gene for mitral valve prolapse. Circ Genom Precis Med. 2019;12: e002497.

316. Mantovani F, Clavel MA, Michelena HI, Suri RM, Schaff HV, EnriquezSarano M. Comprehensive imaging in women with organic mitral regurgitation: implications for clinical outcome. JACC Cardiovasc Imaging. 2016;9:388-396.

317. Asgar AW, Mack MJ, Stone GW. Secondary mitral regurgitation in heart failure: pathophysiology, prognosis, and therapeutic considerations. J Am Coll Cardiol. 2015;65:1231-1248.

318. Bertrand PB, Schwammenthal E, Levine RA, Vandervoort PM. Exercise dynamics in secondary mitral regurgitation: pathophysiology and therapeutic implications. Circulation. 2017;135:297-314.

319. Deferm S, Bertrand PB, Verbrugge FH, Verhaert D, Rega F, Thomas JD, Vandervoort PM. Atrial functional mitral regurgitation: JACC Review Topic of the Week. J Am Coll Cardiol. 2019;73:2465-2476.
320. Bartko PE, Arfsten H, Heitzinger G, Pavo N, Toma A, Strunk G, Hengstenberg C, Hulsmann M, Goliasch G. A unifying concept for the quantitative assessment of secondary mitral regurgitation. J Am Coll Cardiol. 2019;73:2506-2517.

321. Goliasch G, Bartko PE, Pavo N, Neuhold S, Wurm R, Mascherbauer J, Lang IM, Strunk G, Hulsmann M. Refining the prognostic impact of functional mitral regurgitation in chronic heart failure. Eur Heart J. 2018;39:39-46.

322. Michler RE, Smith PK, Parides MK, Ailawadi G, Thourani V, Moskowitz AJ, Acker MA, Hung JW, Chang HL, Perrault LP, Gillinov AM, Argenziano M, Bagiella E, Overbey JR, Moquete EG, Gupta LN, Miller MA, Taddei-Peters WC, Jeffries N, Weisel RD, Rose EA, Gammie JS, DeRose JJ Jr, Puskas JD, Dagenais F, Burks SG, El-Hamamsy I, Milano CA, Atluri P, Voisine P, O'Gara PT, Gelijns AC, CTSN. Twoyear outcomes of surgical treatment of moderate ischemic mitral regurgitation. $N$ Engl JMed. 2016;374:1932-1941.

323. Obadia JF, Messika-Zeitoun D, Leurent G, Iung B, Bonnet G, Piriou N, Lefevre T, Piot C, Rouleau F, Carrie D, Nejjari M, Ohlmann P, Leclercq F, Saint Etienne C, Teiger E, Leroux L, Karam N, Michel N, Gilard M, Donal E, Trochu JN, Cormier B, Armoiry X, Boutitie F, Maucort-Boulch D, Barnel C, Samson G, Guerin P, Vahanian A, Mewton N, MITRA-FR Investigators. Percutaneous repair or medical treatment for secondary mitral regurgitation. N Engl J Med. 2018;379:2297-2306.

324. Cavalcante JL, Kusunose K, Obuchowski NA, Jellis C, Griffin BP, Flamm SD, Kwon DH. Prognostic impact of ischemic mitral regurgitation severity and myocardial infarct quantification by cardiovascular magnetic resonance. JACC Cardiovasc Imaging. 2020;13:1489-1501.

325. Kamperidis V, Marsan NA, Delgado V, Bax JJ. Left ventricular systolic function assessment in secondary mitral regurgitation: left ventricular ejection fraction vs. speckle tracking global longitudinal strain. Eur Heart J. 2016;37:811-816.

326. Namazi F, van der Bijl P, Hirasawa K, Kamperidis V, van Wijngaarden SE, Mertens B, Leon MB, Hahn RT, Stone GW, Narula J, Ajmone Marsan N, Delgado V, Bax JJ. Prognostic value of left ventricular global longitudinal strain in patients with secondary mitral regurgitation. $J$ Am Coll Cardiol. 2020;75:750-758.

327. Kang DH, Park SJ, Shin SH, Hong GR, Lee S, Kim MS, Yun SC, Song JM, Park SW, Kim JJ. Angiotensin receptor neprilysin inhibitor for functional mitral regurgitation. Circulation. 2019;139:1354-1365.

328. Grigioni F, Enriquez-Sarano M, Zehr KJ, Bailey KR, Tajik AJ. Ischemic mitral regurgitation: long-term outcome and prognostic implications with quantitative Doppler assessment. Circulation. 2001;103:1759-1764.

329. Acker MA, Jessup M, Bolling SF, Oh J, Starling RC, Mann DL, Sabbah HN, Shemin R, Kirklin J, Kubo SH. Mitral valve repair in heart failure: five-year follow-up from the mitral valve replacement stratum of the Acorn randomized trial. $J$ Thorac Cardiovasc Surg. 2011;142:569-574, 574 e561.

330. Deja MA, Grayburn PA, Sun B, Rao V, She L, Krejca M, Jain AR, Leng Chua Y, Daly R, Senni M, Mokrzycki K, Menicanti L, Oh JK, Michler R, Wrobel K, Lamy A, Velazquez EJ, Lee KL, Jones RH. Influence of mitral regurgitation repair on survival in the surgical treatment for ischemic heart failure trial. Circulation. 2012;125: 2639-2648.

331. Petrus AHJ, Dekkers OM, Tops LF, Timmer E, Klautz RJM, Braun J. Impact of recurrent mitral regurgitation after mitral valve repair for functional mitral regurgitation: long-term analysis of competing outcomes. Eur Heart J. 2019;40:2206-2214.

332. Harmel EK, Reichenspurner H, Girdauskas E. Subannular reconstruction in secondary mitral regurgitation: a meta-analysis. Heart. 2018;104:1783-1790.

333. Acker MA, Parides MK, Perrault LP, Moskowitz AJ, Gelijns AC, Voisine P, Smith PK, Hung JW, Blackstone EH, Puskas JD, Argenziano M, Gammie JS, Mack M, Ascheim DD, Bagiella E, Moquete EG, Ferguson TB, Horvath KA, Geller NL, Miller MA, Woo YJ, D'Alessandro DA, Ailawadi G, Dagenais F, Gardner TJ, O'Gara PT, Michler RE, Kron IL, CTSN. Mitral-valve repair versus replacement for severe ischemic mitral regurgitation. $N$ Engl J Med. 2014;370:23-32.

334. Mihaljevic T, Lam BK, Rajeswaran J, Takagaki M, Lauer MS, Gillinov AM, Blackstone EH, Lytle BW. Impact of mitral valve annuloplasty combined with revascularization in patients with functional ischemic mitral regurgitation. $\mathrm{J} \mathrm{Am} \mathrm{Coll}$ Cardiol. 2007;49:2191-2201.

335. Wu AH, Aaronson KD, Bolling SF, Pagani FD, Welch K, Koelling TM. Impact of mitral valve annuloplasty on mortality risk in patients with mitral regurgitation and left ventricular systolic dysfunction. J Am Coll Cardiol. 2005;45:381-387.

336. Iung B, Armoiry X, Vahanian A, Boutitie F, Mewton N, Trochu JN, Lefevre T, Messika-Zeitoun D, Guerin P, Cormier B, Brochet E, Thibault H, Himbert D, Thivolet S, Leurent G, Bonnet G, Donal E, Piriou N, Piot C, Habib G, Rouleau F, Carrie D, Nejjari M, Ohlmann P, Saint Etienne C, Leroux L, Gilard M, Samson G, Rioufol G, Maucort-Boulch D, Obadia JF, MITRA-FR Investigators. Percutaneous repair or medical treatment for secondary mitral regurgitation: outcomes at 2 years. Eur J Heart Fail. 2019;21:1619-1627.

337. Stone GW, Lindenfeld J, Abraham WT, Kar S, Lim DS, Mishell JM, Whisenant B, Grayburn PA, Rinaldi M, Kapadia SR, Rajagopal V, Sarembock IJ, Brieke A, Marx SO, 
Cohen DJ, Weissman NJ, Mack MJ, COAPT Investigators. Transcatheter mitral-valve repair in patients with heart failure. $N$ Engl J Med. 2018;379:2307-2318.

338. Mack MJ, Lindenfeld J, Abraham WT, Kar S, Lim DS, Mishell JM, Whisenant BK, Grayburn PA, Rinaldi MJ, Kapadia SR, Rajagopal V, Sarembock IJ, Brieke A, Rogers JH, Marx SO, Cohen DJ, Weissman NJ, Stone GW, COAPT Investigators. 3-Year outcomes of transcatheter mitral valve repair in patients with heart failure. $J \mathrm{Am}$ Coll Cardiol. 2021;77:1029-1040.

339. Giustino G, Lindenfeld J, Abraham WT, Kar S, Lim DS, Grayburn PA, Kapadia SR, Cohen DJ, Kotinkaduwa LN, Weissman NJ, Mack MJ, Stone GW. NYHA functional classification and outcomes after transcatheter mitral valve repair in heart failure: the COAPT Trial. JACC Cardiovasc Interv. 2020;13:2317-2328.

340. Malik UI, Ambrosy AP, Ku IA, Mishell JM, Kar S, Lim DS, Whisenant BK, Cohen DJ, Arnold SV, Kotinkaduwa LN, Lindenfeld J, Abraham WT, Mack MJ, Stone GW. Baseline functional capacity and transcatheter mitral valve repair in heart failure with secondary mitral regurgitation. JACC Cardiovasc Interv. 2020;13: 2331-2341.

341. Kosmidou I, Lindenfeld J, Abraham WT, Kar S, Lim DS, Mishell JM, Whisenant BK, Kipperman RM, Boudoulas KD, Redfors B, Shahim B, Zhang Z, Mack MJ, Stone GW. Transcatheter mitral valve repair in patients with and without cardiac resynchronization therapy: the COAPT Trial. Circ Heart Fail. 2020;13: e007293.

342. Lerakis S, Kini AS, Asch FM, Kar S, Lim DS, Mishell JM, Whisenant B, Grayburn PA, Weissman NJ, Rinaldi MJ, Sharma SK, Kapadia SR, Rajagopal V, Sarembock IJ, Brieke A, Tang GHL, Li D, Crowley A, Lindenfeld J, Abraham WT, Mack MJ, Stone GW. Outcomes of transcatheter mitral valve repair for secondary mitral regurgitation by severity of left ventricular dysfunction. EuroIntervention. 2021;17: e335-e342.

343. Gertz ZM, Herrmann HC, Lim DS, Kar S, Kapadia SR, Reed GW, Puri R, Krishnaswamy A, Gersh BJ, Weissman NJ, Asch FM, Grayburn PA, Kosmidou I, Redfors B, Zhang Z, Abraham WT, Lindenfeld J, Stone GW, Mack MJ. Implications of atrial fibrillation on the mechanisms of mitral regurgitation and response to MitraClip in the COAPT Trial. Circ Cardiovasc Interv. 2021;14:e010300.

344. Messika-Zeitoun D, Iung B, Armoiry X, Trochu JN, Donal E, Habib G, Brochet E, Thibault H, Piriou N, Cormier B, Tribouilloy C, Guerin P, Lefevre T, MaucortBoulch D, Vahanian A, Boutitie F, Obadia JF. Impact of mitral regurgitation severity and left ventricular remodeling on outcome after MitraClip implantation: results from the Mitra-FR Trial. JACC Cardiovasc Imaging. 2021;14:742-752.

345. Grayburn PA, Sannino A, Packer M. Proportionate and disproportionate functional mitral regurgitation: a new conceptual framework that reconciles the results of the MITRA-FR and COAPT trials. JACC Cardiovasc Imaging. 2019;12:353-362.

346. Mack MJ, Abraham WT, Lindenfeld J, Bolling SF, Feldman TE, Grayburn PA, Kapadia SR, McCarthy PM, Lim DS, Udelson JE, Zile MR, Gammie JS, Gillinov AM, Glower DD, Heimansohn DA, Suri RM, Ellis JT, Shu Y, Kar S, Weissman NJ, Stone GW. Cardiovascular outcomes assessment of the MitraClip in patients with heart failure and secondary mitral regurgitation: design and rationale of the COAPT trial. $\mathrm{Am}$ Heart J. 2018;205:1-11.

347. Asch FM, Grayburn PA, Siegel RJ, Kar S, Lim DS, Zaroff JG, Mishell JM, Whisenant B, Mack MJ, Lindenfeld J, Abraham WT, Stone GW, Weissman NJ, COAPT Investigators. Echocardiographic outcomes after transcatheter leaflet approximation in patients with secondary mitral regurgitation: the COAPT Trial. $J$ Am Coll Cardiol. 2019;74:2969-2979.

348. Coats AJS, Anker SD, Baumbach A, Alfieri O, von Bardeleben RS, Bauersachs J, Bax JJ, Boveda S, Celutkiene J, Cleland JG, Dagres N, Deneke T, Farmakis D, Filippatos G, Hausleiter J, Hindricks G, Jankowska EA, Lainscak M, Leclercq C, Lund LH, McDonagh T, Mehra MR, Metra M, Mewton N, Mueller C, Mullens W, Muneretto C, Obadia JF, Ponikowski P, Praz F, Rudolph V, Ruschitzka F, Vahanian A, Windecker S, Zamorano JL, Edvardsen T, Heidbuchel H, Seferovic PM, Prendergast B. The management of secondary mitral regurgitation in patients with heart failure: a joint position statement from the Heart Failure Association (HFA), European Association of Cardiovascular Imaging (EACVI), European Heart Rhythm Association (EHRA), and European Association of Percutaneous Cardiovascular Interventions (EAPCI) of the ESC. Eur Heart J. 2021;42:1254-1269.

349. Pibarot P, Delgado V, Bax JJ. MITRA-FR vs. COAPT: lessons from two trials with diametrically opposed results. Eur Heart J Cardiovasc Imaging. 2019;20: 620-624.

350. Praz F, Grasso C, Taramasso M, Baumbach A, Piazza N, Tamburino C, Windecker S, Maisano F, Prendergast B. Mitral regurgitation in heart failure: time for a rethink. Eur Heart J. 2019;40:2189-2193.

351. Adamo M, Grasso C, Capodanno D, Rubbio AP, Scandura S, Giannini C, Fiorelli F, Fiorina C, Branca L, Brambilla N, Bedogni F, Petronio AS, Curello S, Tamburino C. Five-year clinical outcomes after percutaneous edge-to-edge mitral valve repair: insights from the multicenter GRASP-IT registry. Am Heart J. 2019; 217:32-41.
352. Ailawadi G, Lim DS, Mack MJ, Trento A, Kar S, Grayburn PA, Glower DD, Wang A, Foster E, Qasim A, Weissman NJ, Ellis J, Crosson L, Fan F, Kron IL, Pearson PJ, Feldman T, EVEREST II Investigators. One-year outcomes after MitraClip for functional mitral regurgitation. Circulation. 2019;139:37-47.

353. Iliadis C, Metze C, Korber MI, Baldus S, Pfister R. Impact of COAPT trial exclusion criteria in real-world patients undergoing transcatheter mitral valve repair. Int $J$ Cardiol. 2020;316:189-194.

354. Lindenfeld J, Abraham WT, Grayburn PA, Kar S, Asch FM, Lim DS, Nie H, Singhal P, Sundareswaran KS, Weissman NJ, Mack MJ, Stone GW, Cardiovascular Outcomes Assessment of the MitraClip Percutaneous Therapy for Heart Failure Patients With Functional Mitral Regurgitation Investigators. Association of effective regurgitation orifice area to left ventricular end-diastolic volume ratio with transcatheter mitral valve repair outcomes: a secondary analysis of the COAPT Trial. JAMA Cardiol. 2021;6:427-436.

355. Jung RG, Simard T, Kovach C, Flint K, Don C, Di Santo P, Adamo M, Branca L, Valentini F, Benito-Gonzalez T, Fernandez-Vazquez F, Estevez-Loureiro R, Berardini A, Conti N, Rapezzi C, Biagini E, Parlow S, Shorr R, Levi A, Manovel A, Cardenal-Piris R, Diaz Fernandez J, Shuvy M, Haberman D, Sala A, Alkhouli MA, Marini C, Bargagna M, Schiavi D, Denti P, Markovic S, Buzzatti N, Chan V, Hynes M, Mesana T, Labinaz M, Pappalardo F, Taramasso M, Hibbert B. Transcatheter mitral valve repair in cardiogenic shock and mitral regurgitation: a patient-level, multicenter analysis. JACC Cardiovasc Interv. 2021;14:1-11.

356. Adamo M, Fiorelli F, Melica B, D'Ortona R, Lupi L, Giannini C, Silva G, Fiorina C, Branca L, Chiari E, Chizzola G, Spontoni P, Espada Guerreiro C, Curello S, Petronio AS, Metra M COAPT-like profile predicts long-term outcomes in patients with secondary mitral regurgitation undergoing MitraClip implantation. JACC Cardiovasc Interv. 2021;14:15-25.

357. Iung B, Messika-Zeitoun D, Boutitie F, Trochu JN, Armoiry X, MaucortBoulch D, Obadia JF, Vahanian A. Characteristics and outcome of COAPT-eligible patients in the MITRA-FR Trial. Circulation. 2020;142:2482-2484.

358. Iung B, Vahanian A. Epidemiology of acquired valvular heart disease. Can $J$ Cardiol. 2014;30:962-970.

359. Abramowitz Y, Jilaihawi H, Chakravarty T, Mack MJ, Makkar RR. Mitral annulus calcification. J Am Coll Cardiol. 2015;66:1934-1941.

360. Desnos C, Iung B, Himbert D, Ducrocq G, Urena M, Cormier B, Brochet E, Ou P, Vahanian A, Bouleti C. Temporal trends on percutaneous mitral commissurotomy: 30 years of experience. J Am Heart Assoc. 2019;8: e012031.

361. Andell P, Li X, Martinsson A, Andersson C, Stagmo M, Zoller B, Sundquist K, Smith JG. Epidemiology of valvular heart disease in a Swedish nationwide hospitalbased register study. Heart. 2017;103:1696-1703.

362. Baumgartner H, Hung J, Bermejo J, Chambers JB, Evangelista A, Griffin BP, Iung B, Otto CM, Pellikka PA, Quinones M, EAE/ASE. Echocardiographic assessment of valve stenosis: EAE/ASE recommendations for clinical practice. Eur J Echocardiogr. 2009;10:1-25.

363. Bouleti C, Iung B, Laouenan C, Himbert D, Brochet E, Messika-Zeitoun D, Detaint D, Garbarz E, Cormier B, Michel PL, Mentre F, Vahanian A. Late results of percutaneous mitral commissurotomy up to 20 years: development and validation of a risk score predicting late functional results from a series of 912 patients. Circulation. 2012;125:2119-2127.

364. Nunes MC, Tan TC, Elmariah S, do Lago R, Margey R, Cruz-Gonzalez I, Zheng H, Handschumacher MD, Inglessis I, Palacios IF, Weyman AE, Hung J. The echo score revisited: impact of incorporating commissural morphology and leaflet displacement to the prediction of outcome for patients undergoing percutaneous mitral valvuloplasty. Circulation. 2014;129:886-895.

365. Wilkins GT, Weyman AE, Abascal VM, Block PC, Palacios IF. Percutaneous balloon dilatation of the mitral valve: an analysis of echocardiographic variables related to outcome and the mechanism of dilatation. Br Heart J. 1988;60:299-308.

366. Badheka AO, Shah N, Ghatak A, Patel NJ, Chothani A, Mehta K, Singh V, Patel N, Grover P, Deshmukh A, Panaich SS, Savani GT, Bhalara V, Arora S, Rathod A, Desai H, Kar S, Alfonso C, Palacios IF, Grines C, Schreiber T, Rihal CS, Makkar R, Cohen MG, O'Neill W, de Marchena E. Balloon mitral valvuloplasty in the United States: a 13-year perspective. Am J Med. 2014;127:1126.e1-1126.e12.

367. Tomai F, Gaspardone A, Versaci F, Ghini AS, Altamura L, De Luca L, Gioffre G, Gioffre PA. Twenty year follow-up after successful percutaneous balloon mitral valvuloplasty in a large contemporary series of patients with mitral stenosis. Int J Cardiol. 2014; 177:881-885.

368. Bouleti C, Iung B, Himbert D, Messika-Zeitoun D, Brochet E, Garbarz E, Cormier B, Vahanian A. Relationship between valve calcification and long-term results of percutaneous mitral commissurotomy for rheumatic mitral stenosis. Circ Cardiovasc Interv. 2014;7:381-389.

369. Bouleti C, Iung B, Himbert D, Brochet E, Messika-Zeitoun D, Detaint D, Garbarz E, Cormier B, Vahanian A. Reinterventions after percutaneous mitral 
commissurotomy during long-term follow-up, up to 20 years: the role of repeat percutaneous mitral commissurotomy. Eur Heart J. 2013;34:1923-1930

370. Kim JY, Kim SH, Myong JP, Kim YR, Kim TS, Kim JH, Jang SW, Oh YS, Lee MY, Rho TH. Outcomes of direct oral anticoagulants in patients with mitral stenosis. J Am Coll Cardiol. 2019;73:1123-1131.

371. Song H, Kang DH, Kim JH, Park KM, Song JM, Choi KJ, Hong MK, Chung CH, Song JK, Lee JW, Park SW, Park SJ. Percutaneous mitral valvuloplasty versus surgical treatment in mitral stenosis with severe tricuspid regurgitation. Circulation. 2007;116: I246-250.

372. El Sabbagh A, Reddy YNV, Barros-Gomes S, Borlaug BA, Miranda WR, Pislaru SV, Nishimura RA, Pellikka PA. Low-gradient severe mitral stenosis: hemodynamic profiles, clinical characteristics, and outcomes. $J$ Am Heart Assoc. 2019;8: e010736.

373. Kato N, Padang R, Scott CG, Guerrero M, Pislaru SV, Pellikka PA. The natural history of severe calcific mitral stenosis. J Am Coll Cardiol. 2020;75:3048-3057.

374. Nishimura RA, Vahanian A, Eleid MF, Mack MJ. Mitral valve disease-current management and future challenges. Lancet. 2016;387:1324-1334.

375. Barasch E, Gottdiener JS, Larsen EK, Chaves PH, Newman AB, Manolio TA. Clinical significance of calcification of the fibrous skeleton of the heart and aortosclerosis in community dwelling elderly. The Cardiovascular Health Study (CHS). Am Heart J. 2006;151:39-47.

376. Alexis SL, Malik AH, El-Eshmawi A, George I, Sengupta A, Kodali SK, Hahn RT, Khalique OK, Zaid S, Guerrero M, Bapat VN, Leon MB, Adams DH, Tang GHL. Surgical and transcatheter mitral valve replacement in mitral annular calcification: a systematic review. J Am Heart Assoc. 2021;10: $\mathrm{e} 018514$.

377. Okuno T, Brugger N, Asami M, Heg D, Siontis GCM, Winkel MG, Lanz J, Grani C, Huber A, Stortecky S, George I, Kodali S, Pilgrim T, Windecker S, Khalique OK, Praz F. Clinical impact of mitral calcium volume in patients undergoing transcatheter aortic valve implantation. J Cardiovasc Comput Tomogr. 2021;15: 356-365.

378. Bertrand PB, Churchill TW, Yucel E, Namasivayam M, Bernard S, Nagata Y, He W, Andrews CT, Picard MH, Weyman AE, Levine RA, Hung J. Prognostic importance of the transmitral pressure gradient in mitral annular calcification with associated mitral valve dysfunction. Eur Heart J. 2020;41:4321-4328.

379. Urena M, Himbert D, Brochet E, Carrasco JL, Iung B, Nataf P, Vahanian A. Transseptal transcatheter mitral valve replacement using balloon-expandable transcatheter heart valves: a step-by-step approach. JACC Cardiovasc Interv. 2017;10:1905-1919.

380. Sud K, Agarwal S, Parashar A, Raza MQ, Patel K, Min D, Rodriguez LL, Krishnaswamy A, Mick SL, Gillinov AM, Tuzcu EM, Kapadia SR. Degenerative mitral stenosis: unmet need for percutaneous interventions. Circulation. 2016;133: 1594-1604.

381. Guerrero M, Urena M, Himbert D, Wang DD, Eleid M, Kodali S, George I, Chakravarty T, Mathur M, Holzhey D, Pershad A, Fang HK, O'Hair D, Jones N, Mahadevan VS, Dumonteil N, Rodes-Cabau J, Piazza N, Ferrari E, Ciaburri D, Nejjari M, DeLago A, Sorajja P, Zahr F, Rajagopal V, Whisenant B, Shah PB, Sinning JM, Witkowski A, Eltchaninoff H, Dvir D, Martin B, Attizzani GF, Gaia D, Nunes NSV, Fassa AA, Kerendi F, Pavlides G, Iyer V, Kaddissi G, Witzke C, Wudel J, Mishkel G, Raybuck B, Wang C, Waksman R, Palacios I, Cribier A, Webb J, Bapat V, Reisman M, Makkar R, Leon M, Rihal C, Vahanian A, O’Neill W, Feldman T. 1-Year outcomes of transcatheter mitral valve replacement in patients with severe mitral annular calcification. J Am Coll Cardiol. 2018;71:1841-1853.

382. Yoon SH, Whisenant BK, Bleiziffer S, Delgado V, Dhoble A, Schofer N, Eschenbach L, Bansal E, Murdoch DJ, Ancona M, Schmidt T, Yzeiraj E, Vincent F, Niikura H, Kim WK, Asami M, Unbehaun A, Hirji S, Fujita B, Silaschi M, Tang GHL, Kuwata S, Wong SC, Frangieh AH, Barker CM, Davies JE, Lauten A, Deuschl F, Nombela-Franco L, Rampat R, Nicz PFG, Masson JB, Wijeysundera HC, Sievert H, Blackman DJ, Gutierrez-Ibanes E, Sugiyama D, Chakravarty T, Hildick-Smith D, de Brito FS Jr., Jensen C, Jung C, Smalling RW, Arnold M, Redwood S, Kasel AM, Maisano F, Treede H, Ensminger SM, Kar S, Kaneko T, Pilgrim T, Sorajja P, Van Belle E, Prendergast BD, Bapat V, Modine T, Schofer J, Frerker C, Kempfert J, Attizzani GF, Latib A, Schaefer U, Webb JG, Bax JJ, Makkar RR. Outcomes of transcatheter mitral valve replacement for degenerated bioprostheses, failed annuloplasty rings, and mitral annular calcification. Eur Heart J. 2019;40:441-451.

383. Guerrero M, Vemulapalli S, Xiang Q, Wang DD, Eleid M, Cabalka AK, Sandhu G, Salinger M, Russell H, Greenbaum A, Kodali S, George I, Dvir D, Whisenant B, Russo MJ, Pershad A, Fang K, Coylewright M, Shah P, Babaliaros V, Khan JM, Tommaso C, Saucedo J, Kar S, Makkar R, Mack M, Holmes D, Leon M, Bapat V, Thourani VH, Rihal C, O'Neill W, Feldman T. Thirty-day outcomes of transcatheter mitral valve replacement for degenerated mitral bioprostheses (valve-in-valve), failed surgical rings (valve-in-ring), and native valve with severe mitral annular calcification (valve-in-mitral annular calcification) in the United States: data from the Society of Thoracic Surgeons/American College of Cardiology/Transcatheter Valve Therapy Registry. Circ Cardiovasc Interv. 2020;13: e008425.
384. Wang DD, Guerrero M, Eng MH, Eleid MF, Meduri CU, Rajagopal V, Yadav PK, Fifer MA, Palacios IF, Rihal CS, Feldman TE, O'Neill WW. Alcohol septal ablation to prevent left ventricular outflow tract obstruction during transcatheter mitral valve replacement: first-in-man study. JACC Cardiovasc Interv. 2019;12:1268-1279.

385. Khan JM, Babaliaros VC, Greenbaum AB, Foerst JR, Yazdani S, McCabe JM, Paone G, Eng MH, Leshnower BG, Gleason PT, Chen MY, Wang DD, Tian X, Stine AM, Rogers T, Lederman RJ. Anterior leaflet laceration to prevent ventricular outflow tract obstruction during transcatheter mitral valve replacement. $J$ Am Coll Cardiol. 2019;73:2521-2534.

386. El Sabbagh A, Eleid MF, Foley TA, Al-Hijji MA, Daly RC, Rihal CS, Said SM. Direct transatrial implantation of balloon-expandable valve for mitral stenosis with severe annular calcifications: early experience and lessons learned. Eur J Cardiothorac Surg. 2018;53:162-169.

387. Praz F, Khalique OK, Lee R, Veeragandham R, Russell H, Guerrero M, Islam AM, Deaton DW, Kaneko T, Kodali SK, Leon MB, Bapat V, Takayama H, Borger MA, George I. Transatrial implantation of a transcatheter heart valve for severe mitral annular calcification. J Thorac Cardiovasc Surg. 2018;156:132-142.

388. Sorajja P, Gossl M, Babaliaros V, Rizik D, Conradi L, Bae R, Burke RF, Schafer U, Lisko JC, Riley RD, Guyton R, Dumonteil N, Berthoumieu P, Tchetche D, Blanke P, Cavalcante JL, Sun B. Novel transcatheter mitral valve prosthesis for patients with severe mitral annular calcification. J Am Coll Cardiol. 2019;74:1431-1440

389. Topilsky Y, Maltais S, Medina Inojosa J, Oguz D, Michelena H, Maalouf J, Mahoney DW, Enriquez-Sarano M. Burden of tricuspid regurgitation in patients diagnosed in the community setting. JACC Cardiovasc Imaging. 2019;12:433-442.

390. Song H, Kim MJ, Chung CH, Choo SJ, Song MG, Song JM, Kang DH, Lee JW, Song JK. Factors associated with development of late significant tricuspid regurgitation after successful left-sided valve surgery. Heart. 2009;95:931-936.

391. Kwak JJ, Kim YJ, Kim MK, Kim HK, Park JS, Kim KH, Kim KB, Ahn H, Sohn DW, Oh BH, Park YB. Development of tricuspid regurgitation late after leftsided valve surgery: a single-center experience with long-term echocardiographic examinations. Am Heart $J$. 2008;155:732-737.

392. Ortiz-Leon XA, Posada-Martinez EL, Trejo-Paredes MC, Ivey-Miranda JB, Pereira J, Crandall I, DaSilva P, Bouman E, Brooks A, Gerardi C, Ugonabo I, Chen W, Houle H, Akar JG, Lin BA, McNamara RL, Lombo-Lievano B, Arias-Godinez JA, Sugeng $\mathrm{L}$. Understanding tricuspid valve remodelling in atrial fibrillation using threedimensional echocardiography. Eur Heart J Cardiovasc Imaging. 2020;21:747-755.

393. Kim JB, Spevack DM, Tunick PA, Bullinga JR, Kronzon I, Chinitz LA, Reynolds HR. The effect of transvenous pacemaker and implantable cardioverter defibrillator lead placement on tricuspid valve function: an observational study. J Am Soc Echocardiogr. 2008;21:284-287.

394. Hoke U, Auger D, Thijssen J, Wolterbeek R, van der Velde ET, Holman ER, Schalij MJ, Bax JJ, Delgado V, Marsan NA. Significant lead-induced tricuspid regurgitation is associated with poor prognosis at long-term follow-up. Heart. 2014;100: 960-968.

395. Anvardeen K, Rao R, Hazra S, Hay K, Dai H, Stoyanov N, Birnie D, Dwivedi G, Chan KL. Prevalence and significance of tricuspid regurgitation post-endocardial lead placement. JACC Cardiovasc Imaging. 2019;12:562-564.

396. Prihadi EA, van der Bijl P, Gursoy E, Abou R, Mara Vollema E, Hahn RT, Stone GW, Leon MB, Ajmone Marsan N, Delgado V, Bax JJ. Development of significant tricuspid regurgitation over time and prognostic implications: new insights into natural history. Eur Heart J. 2018;39:3574-3581.

397. Benfari G, Antoine C, Miller WL, Thapa P, Topilsky Y, Rossi A, Michelena HI, Pislaru S, Enriquez-Sarano M. Excess mortality associated with functional tricuspid regurgitation complicating heart failure with reduced ejection fraction. Circulation. 2019;140:196-206.

398. Dietz MF, Prihadi EA, van der Bijl P, Goedemans L, Mertens BJA, Gursoy E, van Genderen OS, Ajmone Marsan N, Delgado V, Bax JJ. Prognostic implications of right ventricular remodeling and function in patients with significant secondary tricuspid regurgitation. Circulation. 2019;140:836-845.

399. Muraru D, Badano LP, Nagata Y, Surkova E, Nabeshima Y, Genovese D, Otsuji Y, Guida V, Azzolina D, Palermo C, Takeuchi M. Development and prognostic validation of partition values to grade right ventricular dysfunction severity using 3D echocardiography. Eur Heart J Cardiovasc Imaging. 2020;21:10-21.

400. Park JB, Lee SP, Lee JH, Yoon YE, Park EA, Kim HK, Lee W, Kim YJ, Cho GY, Sohn DW. Quantification of right ventricular volume and function using single-beat three-dimensional echocardiography: a validation study with cardiac magnetic resonance. J Am Soc Echocardiogr. 2016;29:392-401.

401. Grothues F, Moon JC, Bellenger NG, Smith GS, Klein HU, Pennell DJ. Interstudy reproducibility of right ventricular volumes, function, and mass with cardiovascular magnetic resonance. Am Heart J. 2004;147:218-223.

402. Song JM, Jang MK, Choi YS, Kim YJ, Min SY, Kim DH, Kang DH, Song JK. The vena contracta in functional tricuspid regurgitation: a real-time three-dimensional color Doppler echocardiography study. J Am Soc Echocardiogr. 2011;24:663-670. 
403. de Agustin JA, Viliani D, Vieira C, Islas F, Marcos-Alberca P, Gomez de Diego JJ, Nunez-Gil IJ, Almeria C, Rodrigo JL, Luaces M, Garcia-Fernandez MA, Macaya C, Perez de Isla L. Proximal isovelocity surface area by single-beat three-dimensional color Doppler echocardiography applied for tricuspid regurgitation quantification. $J$ Am Soc Echocardiogr. 2013;26:1063-1072.

404. Chen TE, Kwon SH, Enriquez-Sarano M, Wong BF, Mankad SV. Threedimensional color Doppler echocardiographic quantification of tricuspid regurgitation orifice area: comparison with conventional two-dimensional measures. $J$ Am Soc Echocardiogr. 2013;26:1143-1152.

405. Dahou A, Ong G, Hamid N, Avenatti E, Yao J, Hahn RT. Quantifying tricuspid regurgitation severity: a comparison of proximal isovelocity surface area and novel quantitative Doppler methods. JACC Cardiovasc Imaging. 2019;12:560-562.

406. Utsunomiya H, Harada Y, Susawa H, Takahari K, Ueda Y, Izumi K, Itakura K, Ikenaga H, Hidaka T, Fukuda Y, Shiota T, Kihara Y. Comprehensive evaluation of tricuspid regurgitation location and severity using vena contracta analysis: a color Doppler three-dimensional transesophageal echocardiographic study. J Am Soc Echocardiogr. 2019;32:1526-1537.e2.

407. Hahn RT, Zamorano JL. The need for a new tricuspid regurgitation grading scheme. Eur Heart J Cardiovasc Imaging. 2017;18:1342-1343.

408. Nickenig G, Weber M, Lurz P, von Bardeleben RS, Sitges M, Sorajja P, Hausleiter J, Denti P, Trochu JN, Nabauer M, Dahou A, Hahn RT. Transcatheter edgeto-edge repair for reduction of tricuspid regurgitation: 6-month outcomes of the TRILUMINATE single-arm study. Lancet. 2019;394:2002-2011.

409. Nickenig G, Weber M, Schueler R, Hausleiter J, Nabauer M, von Bardeleben RS, Sotiriou E, Schafer U, Deuschl F, Kuck KH, Kreidel F, Juliard JM, Brochet E, Latib A, Agricola E, Baldus S, Friedrichs K, Vandrangi P, Verta P, Hahn RT, Maisano F. 6-Month outcomes of tricuspid valve reconstruction for patients with severe tricuspid regurgitation. J Am Coll Cardiol. 2019;73:1905-1915.

410. Santoro C, Marco Del Castillo A, Gonzalez-Gomez A, Monteagudo JM, Hinojar R, Lorente A, Abellas M, Vieitez JM, Garcia Martin A, Casas Rojo E, Ruiz S, Barrios V, Luis Moya J, Jimenez-Nacher JJ, Zamorano Gomez JL, Fernandez-Golfin C. Mid-term outcome of severe tricuspid regurgitation: are there any differences according to mechanism and severity? Eur Heart J Cardiovasc Imaging. 2019;20: 1035-1042.

411. Miura M, Alessandrini H, Alkhodair A, Attinger-Toller A, Biasco L, Lurz P, Braun D, Brochet E, Connelly KA, de Bruijn S, Denti P, Deuschl F, Estevez-Loureiro R, Fam N, Frerker C, Gavazzoni M, Hausleiter J, Himbert D, Ho E, Juliard JM, Kaple R, Besler C, Kodali S, Kreidel F, Kuck KH, Latib A, Lauten A, Monivas V, Mehr M, Muntane-Carol G, Nazif T, Nickenig G, Pedrazzini G, Philippon F, Pozzoli A, Praz F, Puri R, Rodes-Cabau J, Schafer U, Schofer J, Sievert H, Tang GHL, Thiele H, Rommel KP, Vahanian A, Von Bardeleben RS, Webb JG, Weber M, Windecker S, Winkel M, Zuber M, Leon MB, Maisano F, Hahn RT, Taramasso M, TriValve Investigators. Impact of massive or torrential tricuspid regurgitation in patients undergoing transcatheter tricuspid valve intervention. JACC Cardiovasc Interv. 2020;13: 1999-2009.

412. Peri Y, Sadeh B, Sherez C, Hochstadt A, Biner S, Aviram G, Ingbir M, Nachmany I, Topaz G, Flint N, Keren G, Topilsky Y. Quantitative assessment of effective regurgitant orifice: impact on risk stratification, and cut-off for severe and torrential tricuspid regurgitation grade. Eur Heart J Cardiovasc Imaging. 2020;21:768-776.

413. Stocker TJ, Hertell H, Orban M, Braun D, Rommel KP, Ruf T, Ong G, Nabauer M, Deseive S, Fam N, von Bardeleben RS, Thiele H, Massberg S, Lurz P, Hausleiter J. Cardiopulmonary hemodynamic profile predicts mortality after transcatheter tricuspid valve repair in chronic heart failure. JACC Cardiovasc Interv. 2021;14:29-38.

414. Nath J, Foster E, Heidenreich PA. Impact of tricuspid regurgitation on long-term survival. J Am Coll Cardiol. 2004;43:405-409.

415. Topilsky Y, Nkomo VT, Vatury O, Michelena HI, Letourneau T, Suri RM, Pislaru S, Park S, Mahoney DW, Biner S, Enriquez-Sarano M. Clinical outcome of isolated tricuspid regurgitation. JACC Cardiovasc Imaging. 2014;7:1185-1194.

416. Chorin E, Rozenbaum Z, Topilsky Y, Konigstein M, Ziv-Baran T, Richert E, Keren G, Banai S. Tricuspid regurgitation and long-term clinical outcomes. Eur Heart J Cardiovasc Imaging. 2020;21:157-165.

417. Topilsky Y, Inojosa JM, Benfari G, Vaturi O, Maltais S, Michelena H, Mankad S, Enriquez-Sarano M. Clinical presentation and outcome of tricuspid regurgitation in patients with systolic dysfunction. Eur Heart J. 2018;39:3584-3592.

418. Kadri AN, Menon V, Sammour YM, Gajulapalli RD, Meenakshisundaram C, Nusairat L, Mohananey D, Hernandez AV, Navia J, Krishnaswamy A, Griffin B, Rodriguez L, Harb SC, Kapadia S. Outcomes of patients with severe tricuspid regurgitation and congestive heart failure. Heart. 2019;105:1813-1817.

419. Stuge O, Liddicoat J. Emerging opportunities for cardiac surgeons within structural heart disease. J Thorac Cardiovasc Surg. 2006;132:1258-1261.

420. Kilic A, Saha-Chaudhuri P, Rankin JS, Conte JV. Trends and outcomes of tricuspid valve surgery in North America: an analysis of more than 50,000 patients from the Society of Thoracic Surgeons database. Ann Thorac Surg. 2013;96:1546-1552.
421. Dreyfus J, Ghalem N, Garbarz E, Cimadevilla C, Nataf P, Vahanian A, Caranhac G, Messika-Zeitoun D. Timing of referral of patients with severe isolated tricuspid valve regurgitation to surgeons (from a French nationwide database). Am J Cardiol. 2018;122:323-326.

422. Antunes MJ, Rodriguez-Palomares J, Prendergast B, De Bonis M, Rosenhek R, Al-Attar N, Barili F, Casselman F, Folliguet T, Iung B, Lancellotti P, Muneretto C, Obadia JF, Pierard L, Suwalski P, Zamorano P, ESC Working Groups of Cardiovascular Surgery and Valvular Heart Disease. Management of tricuspid valve regurgitation: position statement of the European Society of Cardiology Working Groups of Cardiovascular Surgery and Valvular Heart Disease. Eur J Cardiothorac Surg. 2017;52:1022-1030.

423. Dreyfus GD, Corbi PJ, Chan KM, Bahrami T. Secondary tricuspid regurgitation or dilatation: which should be the criteria for surgical repair? Ann Thorac Surg. 2005;79:127-132.

424. Van de Veire NR, Braun J, Delgado V, Versteegh MI, Dion RA, Klautz RJ, Bax JJ. Tricuspid annuloplasty prevents right ventricular dilatation and progression of tricuspid regurgitation in patients with tricuspid annular dilatation undergoing mitral valve repair. J Thorac Cardiovasc Surg. 2011;141:1431-1439.

425. Chikwe J, Itagaki S, Anyanwu A, Adams DH. Impact of concomitant tricuspid annuloplasty on tricuspid regurgitation, right ventricular function, and pulmonary artery hypertension after repair of mitral valve prolapse. J Am Coll Cardiol. 2015;65: 1931-1938.

426. Badhwar V, Rankin JS, He M, Jacobs JP, Furnary AP, Fazzalari FL, O'Brien S, Gammie JS, Shahian DM. Performing concomitant tricuspid valve repair at the time of mitral valve operations is not associated with increased operative mortality. Ann Thorac Surg. 2017;103:587-593.

427. Brescia AA, Ward ST, Watt TMF, Rosenbloom LM, Baker M, Khan S, Ziese E, Romano MA, Bolling SF, Michigan Mitral Research Group. Outcomes of guidelinedirected concomitant annuloplasty for functional tricuspid regurgitation. Ann Thorac Surg. 2020;109:1227-1232.

428. Axtell AL, Bhambhani V, Moonsamy P, Healy EW, Picard MH, Sundt TM 3rd, Wasfy JH. Surgery does not improve survival in patients with isolated severe tricuspid regurgitation. J Am Coll Cardiol. 2019;74:715-725.

429. Zack CJ, Fender EA, Chandrashekar P, Reddy YNV, Bennett CE, Stulak JM, Miller VM, Nishimura RA. National trends and outcomes in isolated tricuspid valve surgery. J Am Coll Cardiol. 2017;70:2953-2960.

430. Dhoble A, Zhao Y, Vejpongsa P, Loghin C, Smalling RW, Estrera A, Nguyen TC. National 10-year trends and outcomes of isolated and concomitant tricuspid valve surgery. J Cardiovasc Surg (Torino). 2019;60:119-127.

431. Alqahtani F, Berzingi CO, Aljohani S, Hijazi M, Al-Hallak A, Alkhouli M. Contemporary trends in the use and outcomes of surgical treatment of tricuspid regurgitation. J Am Heart Assoc. 2017;6.

432. Dreyfus J, Flagiello M, Bazire B, Eggenspieler F, Viau F, Riant E, Mbaki Y, Bohbot Y, Eyharts D, Senage T, Dubrulle H, Nicol M, Doguet F, Nguyen V, Coisne A, Le Tourneau T, Lavie-Badie Y, Tribouilloy C, Donal E, Tomasi J, Habib G, SeltonSuty C, Raffoul R, Iung B, Obadia JF, Messika-Zeitoun D. Isolated tricuspid valve surgery: impact of aetiology and clinical presentation on outcomes. Eur Heart $J$. 2020;41:4304-4317.

433. Hamandi M, Smith RL, Ryan WH, Grayburn PA, Vasudevan A, George TJ, DiMaio JM, Hutcheson KA, Brinkman W, Szerlip M, Moore DO, Mack MJ. Outcomes of isolated tricuspid valve surgery have improved in the modern era. Ann Thorac Surg. 2019;108:11-15.

434. Dietz MF, Prihadi EA, van der Bijl P, Ajmone Marsan N, Delgado V, Bax JJ. Prognostic implications of staging right heart failure in patients with significant secondary tricuspid regurgitation. JACC Heart Fail. 2020;8:627-636.

435. McCarthy PM, Bhudia SK, Rajeswaran J, Hoercher KJ, Lytle BW, Cosgrove DM, Blackstone EH. Tricuspid valve repair: durability and risk factors for failure. J Thorac Cardiovasc Surg. 2004;127:674-685.

436. Chang BC, Lim SH, Yi G, Hong YS, Lee S, Yoo KJ, Kang MS, Cho BK. Longterm clinical results of tricuspid valve replacement. Ann Thorac Surg. 2006;81: 1317-1323.

437. Glikson M, Nielsen JC, Michowitz Y, Kronborg MB, Auricchio A, Barbash IM, Barrabés JA, Boriani G, Braunschweig F, Brignole M, Burri H, Coats AJS, Deharo JC, Delgado V, Diller GP, Israel CW, Keren A, Knops RE, Kotecha D, Leclercq C, Merkely B, Starck C, Thylén I, Tolosana JM, ESC Scientific Document Group. 2021 ESC Guidelines on cardiac pacing and cardiac resynchronization therapy. Eur Heart J 2021; doi:10.1093/eurheartj/ehab364.

438. Fam NP, Braun D, von Bardeleben RS, Nabauer M, Ruf T, Connelly KA, Ho E, Thiele H, Lurz P, Weber M, Nickenig G, Narang A, Davidson CJ, Hausleiter J. Compassionate use of the PASCAL transcatheter valve repair system for severe tricuspid regurgitation: a multicenter, observational, first-in-human experience. $J A C C$ Cardiovasc Interv. 2019;12:2488-2495. 
439. Lurz P, Stephan von Bardeleben R, Weber M, Sitges M, Sorajja P, Hausleiter J, Denti P, Trochu JN, Nabauer M, Tang GHL, Biaggi P, Ying SW, Trusty PM, Dahou A, Hahn RT, Nickenig G, TRILUMINATE Investigators. Transcatheter edge-to-edge repair for treatment of tricuspid regurgitation. J Am Coll Cardiol. 2021;77:229-239.

440. Kodali S, Hahn RT, Eleid MF, Kipperman R, Smith R, Lim DS, Gray WA, Narang A, Pislaru SV, Koulogiannis K, Grayburn P, Fowler D, Hawthorne K, Dahou A, Deo SH, Vandrangi P, Deuschl F, Mack MJ, Leon MB, Feldman T, Davidson CJ, CLASP TR EFS Investigators. Feasibility study of the transcatheter valve repair system for severe tricuspid regurgitation. J Am Coll Cardiol. 2021;77:345-356.

441. Nickenig G, Weber M, Schuler R, Hausleiter J, Nabauer M, von Bardeleben RS, Sotiriou E, Schafer U, Deuschl F, Alessandrini H, Kreidel F, Juliard JM, Brochet E, Latib A, Montorfano M, Agricola E, Baldus S, Friedrichs KP, Deo SH, Gilmore SY, Feldman T, Hahn RT, Maisano F. Tricuspid valve repair with the Cardioband system: two-year outcomes of the multicentre, prospective TRI-REPAIR study. EuroIntervention. 2021;16: e1264-e1271.

442. Hahn RT, Kodali S, Fam N, Bapat V, Bartus K, Rodes-Cabau J, Dagenais F, Estevez-Loureiro R, Forteza A, Kapadia S, Latib A, Maisano F, McCarthy P, Navia J, Ong G, Peterson M, Petrossian G, Pozzoli A, Reinartz M, Ricciardi MJ, Robinson N, Sievert H, Taramasso M, Agarwal V, Bedard E, Tarantini G, Colli A. Early multinational experience of transcatheter tricuspid valve replacement for treating severe tricuspid regurgitation. JACC Cardiovasc Interv. 2020;13:2482-2493.

443. Fam NP, von Bardeleben RS, Hensey M, Kodali SK, Smith RL, Hausleiter J, Ong G, Boone R, Ruf T, George I, Szerlip M, Nabauer M, Ali FM, Moss R, Bapat V, Schnitzler K, Kreidel F, Ye J, Deva DP, Mack MJ, Grayburn PA, Peterson MD, Leon MB, Hahn RT, Webb JG. Transfemoral transcatheter tricuspid valve replacement with the EVOQUE system: a multicenter, observational, first-in-human experience. JACC Cardiovasc Interv. 2021;14:501-511.

444. Lu FL, Ma Y, An Z, Cai CL, Li BL, Song ZG, Han L, Wang J, Qiao F, Xu ZY. First-in-man experience of transcatheter tricuspid valve replacement with lux-valve in high-risk tricuspid regurgitation patients. JACC Cardiovasc Interv. 2020;13: 1614-1616.

445. Rommel KP, Besler C, Noack T, Blazek S, von Roeder M, Fengler K, Ender J, Gutberlet M, Desch S, Borger MA, Thiele H, Lurz P. Physiological and clinical consequences of right ventricular volume overload reduction after transcatheter treatment for tricuspid regurgitation. JACC Cardiovasc Interv. 2019;12:1423-1434.

446. Montalto C, Sticchi A, Crimi G, Laricchia A, Khokhar A, Giannini F, Ferlini M, Colombo A, Latib A, Mangieri A. Functional and echocardiographic improvement after transcatheter repair for tricuspid regurgitation: a systematic review and pooled analysis. JACC Cardiovasc Interv. 2020;13:2719-2729.

447. Taramasso M, Benfari G, van der Bijl P, Alessandrini H, Attinger-Toller A, Biasco L, Lurz P, Braun D, Brochet E, Connelly KA, de Bruijn S, Denti P, Deuschl F, Estevez-Loureiro R, Fam N, Frerker C, Gavazzoni M, Hausleiter J, Ho E, Juliard JM, Kaple R, Besler C, Kodali S, Kreidel F, Kuck KH, Latib A, Lauten A, Monivas V, Mehr M, Muntane-Carol G, Nazif T, Nickening G, Pedrazzini G, Philippon F, Pozzoli A, Praz F, Puri R, Rodes-Cabau J, Schafer U, Schofer J, Sievert H, Tang GHL, Thiele H, Topilsky Y, Rommel KP, Delgado V, Vahanian A, Von Bardeleben RS, Webb JG, Weber M, Windecker S, Winkel M, Zuber M, Leon MB, Hahn RT, Bax JJ, EnriquezSarano M, Maisano F. Transcatheter versus medical treatment of patients with symptomatic severe tricuspid regurgitation. J Am Coll Cardiol. 2019;74:2998-3008.

448. Prihadi EA, Delgado V, Hahn RT, Leipsic J, Min JK, Bax JJ. Imaging needs in novel transcatheter tricuspid valve interventions. JACC Cardiovasc Imaging. 2018;11:736-754.

449. Hahn RT. State-of-the-art review of echocardiographic imaging in the evaluation and treatment of functional tricuspid regurgitation. Circ Cardiovasc Imaging. 2016;9. 450. Utsunomiya H, Itabashi Y, Mihara H, Berdejo J, Kobayashi S, Siegel RJ, Shiota T. Functional tricuspid regurgitation caused by chronic atrial fibrillation: a real-time 3-dimensional transesophageal echocardiography study. Circ Cardiovasc Imaging. $2017 ; 10$.

451. Filsoufi F, Anyanwu AC, Salzberg SP, Frankel T, Cohn LH, Adams DH. Longterm outcomes of tricuspid valve replacement in the current era. Ann Thorac Surg. 2005;80:845-850

452. Yeter E, Ozlem K, Kilic H, Ramazan A, Acikel S. Tricuspid balloon valvuloplasty to treat tricuspid stenosis. J Heart Valve Dis. 2010;19:159-160.

453. Unger P, Pibarot P, Tribouilloy C, Lancellotti P, Maisano F, Iung B, Pierard L, European Society of Cardiology Council on Valvular Heart Disease. Multiple and mixed valvular heart diseases. Circ Cardiovasc Imaging. 2018;11: e007862.

454. Egbe AC, Luis SA, Padang R, Warnes CA. Outcomes in moderate mixed aortic valve disease: is it time for a paradigm shift? J Am Coll Cardiol. 2016;67:2321-2329. 455. Egbe AC, Poterucha JT, Warnes CA. Mixed aortic valve disease: midterm outcome and predictors of adverse events. Eur Heart J. 2016;37:2671-2678.

456. Zilberszac R, Gabriel H, Schemper M, Zahler D, Czerny M, Maurer G, Rosenhek R. Outcome of combined stenotic and regurgitant aortic valve disease. $J$ Am Coll Cardiol. 2013;61:1489-1495.
457. Unger P, Tribouilloy C. Aortic stenosis with other concomitant valvular disease: aortic regurgitation, mitral regurgitation, mitral stenosis, or tricuspid regurgitation. Cardiol Clin. 2020;38:33-46.

458. Philip JL, Zens T, Lozonschi L, De Oliveira NC, Osaki S, Kohmoto T, Akhter SA, Tang PC. Outcomes of surgical aortic valve replacement for mixed aortic valve disease. $J$ Thorac Dis. 2018;10:4042-4051.

459. Chahine J, Kadri AN, Gajulapalli RD, Krishnaswamy A, Mick S, Perez O, Lak H, Nair RM, Montane B, Tak J, Tuzcu EM, Griffin B, Svensson LG, Harb SC, Kapadia SR. Outcomes of transcatheter aortic valve replacement in mixed aortic valve disease. JACC Cardiovasc Interv. 2019;12:2299-2306.

460. Yang LT, Enriquez-Sarano M, Scott CG, Padang R, Maalouf JF, Pellikka PA, Michelena HI. Concomitant mitral regurgitation in patients with chronic aortic regurgitation. J Am Coll Cardiol. 2020;76:233-246.

461. Mehr M, Karam N, Taramasso M, Ouarrak T, Schneider S, Lurz P, von Bardeleben RS, Fam N, Pozzoli A, Lubos E, Boekstegers P, Schillinger W, Plicht B, Eggebrecht H, Baldus S, Senges J, Maisano F, Hausleiter J, TriValve, TRAMI Investigators. Combined tricuspid and mitral versus isolated mitral valve repair for severe MR and TR: an analysis from the TriValve and TRAMI registries. JACC Cardiovasc Interv. 2020;13:543-550.

462. Goldstone AB, Chiu P, Baiocchi M, Lingala B, Patrick WL, Fischbein MP, Woo YJ. Mechanical or biologic prostheses for aortic-valve and mitral-valve replacement. $N$ Engl J Med. 2017;377:1847-1857.

463. Head SJ, Celik M, Kappetein AP. Mechanical versus bioprosthetic aortic valve replacement. Eur Heart J. 2017;38:2183-2191.

464. Diaz R, Hernandez-Vaquero D, Alvarez-Cabo R, Avanzas P, Silva J, Moris C, Pascual I. Long-term outcomes of mechanical versus biological aortic valve prosthesis: systematic review and meta-analysis. J Thorac Cardiovasc Surg. 2019;158:706-714.e18. 465. David TE, Ouzounian M, David CM, Lafreniere-Roula M, Manlhiot C. Late results of the Ross procedure. J Thorac Cardiovasc Surg. 2019;157:201-208.

466. Malik AH, Yandrapalli S, Aronow WS, Panza JA, Cooper HA. Oral anticoagulants in atrial fibrillation with valvular heart disease and bioprosthetic heart valves. Heart. 2019;105:1432-1436.

467. Duan L, Doctor JN, Adams JL, Romley JA, Nguyen LA, An J, Lee MS. Comparison of direct oral anticoagulants versus warfarin in patients with atrial fibrillation and bioprosthetic heart valves. Am J Cardiol. 2021;146:22-28.

468. Pasciolla S, Zizza LF, Le T, Wright K. Comparison of the efficacy and safety of direct oral anticoagulants and warfarin after bioprosthetic valve replacements. Clin Drug Investig. 2020;40:839-845.

469. Russo V, Carbone A, Attena E, Rago A, Mazzone C, Proietti R, Parisi V, Scotti A, Nigro G, Golino P, D'Onofrio A. Clinical benefit of direct oral anticoagulants versus vitamin $\mathrm{K}$ antagonists in patients with atrial fibrillation and bioprosthetic heart valves. Clin Ther. 2019;41:2549-2557.

470. Capodanno D, Petronio AS, Prendergast B, Eltchaninoff H, Vahanian A, Modine T, Lancellotti P, Sondergaard L, Ludman PF, Tamburino C, Piazza N, Hancock J, Mehilli J, Byrne RA, Baumbach A, Kappetein AP, Windecker S, Bax J, Haude M. Standardized definitions of structural deterioration and valve failure in assessing long-term durability of transcatheter and surgical aortic bioprosthetic valves: a consensus statement from the European Association of Percutaneous Cardiovascular Interventions (EAPCI) endorsed by the European Society of Cardiology (ESC) and the European Association for Cardio-Thoracic Surgery (EACTS ). Eur Heart J. 2017;38: $3382-3390$

471. Zoghbi WA, Chambers JB, Dumesnil JG, Foster E, Gottdiener JS, Grayburn PA, Khandheria BK, Levine RA, Marx GR, Miller FA Jr., Nakatani S, Quinones MA, Rakowski H, Rodriguez LL, Swaminathan M, Waggoner AD, Weissman NJ, Zabalgoitia M, American Society of Echocardiography's G, Standards C, Task Force on Prosthetic V, American College of Cardiology Cardiovascular Imaging C, Cardiac Imaging Committee of the American Heart A, European Association of E, European Society of C, Japanese Society of E, Canadian Society of E, American College of Cardiology F, American Heart A, European Association of E, European Society of C, Japanese Society of E, Canadian Society of E. Recommendations for evaluation of prosthetic valves with echocardiography and Doppler ultrasound: a report from the American Society of Echocardiography's Guidelines and Standards Committee and the Task Force on Prosthetic Valves, developed in conjunction with the American College of Cardiology Cardiovascular Imaging Committee, Cardiac Imaging Committee of the American Heart Association, the European Association of Echocardiography, a registered branch of the European Society of Cardiology, the Japanese Society of Echocardiography and the Canadian Society of Echocardiography, endorsed by the American College of Cardiology Foundation, American Heart Association, European Association of Echocardiography, a registered branch of the European Society of Cardiology, the Japanese Society of Echocardiography, and Canadian Society of Echocardiography. J Am Soc Echocardiogr. 2009;22:975-1014.

472. Cannegieter SC, Rosendaal FR, Briet E. Thromboembolic and bleeding complications in patients with mechanical heart valve prostheses. Circulation. 1994;89: 635-641. 
473. Mok CK, Boey J, Wang R, Chan TK, Cheung KL, Lee PK, Chow J, Ng RP, Tse TF. Warfarin versus dipyridamole-aspirin and pentoxifylline-aspirin for the prevention of prosthetic heart valve thromboembolism: a prospective randomized clinical trial. Circulation. 1985;72:1059-1063.

474. Eikelboom JW, Connolly SJ, Brueckmann M, Granger CB, Kappetein AP, Mack MJ, Blatchford J, Devenny K, Friedman J, Guiver K, Harper R, Khder Y, Lobmeyer MT, Maas H, Voigt JU, Simoons ML, Van de Werf F, RE-ALIGN Investigators. Dabigatran versus warfarin in patients with mechanical heart valves. $N$ Engl J Med. 2013;369:1206-1214.

475. Iung B, Rodes-Cabau J. The optimal management of anti-thrombotic therapy after valve replacement: certainties and uncertainties. Eur Heart J. 2014;35:2942-2949.

476. Caldeira D, David C, Santos AT, Costa J, Pinto FJ, Ferreira JJ. Efficacy and safety of low molecular weight heparin in patients with mechanical heart valves: systematic review and meta-analysis. J Thromb Haemost. 2014;12:650-659.

477. Laffort P, Roudaut R, Roques X, Lafitte S, Deville C, Bonnet J, Baudet E. Early and long-term (one-year) effects of the association of aspirin and oral anticoagulant on thrombi and morbidity after replacement of the mitral valve with the St. Jude medical prosthesis: a clinical and transesophageal echocardiographic study. $J$ Am Coll Cardiol. 2000;35:739-746.

478. Sousa-Uva M, Head SJ, Milojevic M, Collet JP, Landoni G, Castella M, Dunning J, Gudbjartsson T, Linker NJ, Sandoval E, Thielmann M, Jeppsson A, Landmesser U. 2017 EACTS Guidelines on perioperative medication in adult cardiac surgery. Eur J Cardiothorac Surg. 2018;53:5-33.

479. Butchart EG, Gohlke-Barwolf C, Antunes MJ, Tornos P, De Caterina R, Cormier B, Prendergast B, Iung B, Bjornstad H, Leport C, Hall RJ, Vahanian A, Working Groups on Valvular Heart Disease; Thrombosis and Cardiac Rehabilitation and Exercise Physiology, European Society of Cardiology. Recommendations for the management of patients after heart valve surgery. Eur Heart J. 2005;26:2463-2471.

480. Torella M, Torella D, Chiodini P, Franciulli M, Romano G, De Santo L, De Feo M, Amarelli C, Sasso FC, Salvatore T, Ellison GM, Indolfi C, Cotrufo M, Nappi G. LOWERing the INtensity of oral anticoaGulant Therapy in patients with bileaflet mechanical aortic valve replacement: results from the "LOWERING-IT" Trial. Am Heart J. 2010;160:171-178.

481. Puskas J, Gerdisch M, Nichols D, Quinn R, Anderson C, Rhenman B, Fermin L, McGrath M, Kong B, Hughes C, Sethi G, Wait M, Martin T, Graeve A, PROACT Investigators. Reduced anticoagulation after mechanical aortic valve replacement: interim results from the prospective randomized on- $X$ valve anticoagulation clinical trial randomized Food and Drug Administration investigational device exemption trial. J Thorac Cardiovasc Surg. 2014;147:1202-1210; discussion 1210-1201.

482. Heneghan C, Ward A, Perera R, Self-Monitoring Trialist C, Bankhead C, Fuller A, Stevens R, Bradford K, Tyndel S, Alonso-Coello P, Ansell J, Beyth R, Bernardo A, Christensen TD, Cromheecke ME, Edson RG, Fitzmaurice D, Gadisseur AP, GarciaAlamino JM, Gardiner C, Hasenkam JM, Jacobson A, Kaatz S, Kamali F, Khan TI, Knight E, Kortke H, Levi M, Matchar D, Menendez-Jandula B, Rakovac I, Schaefer C, Siebenhofer A, Souto JC, Sunderji R, Gin K, Shalansky K, Voller H, Wagner O, Zittermann A. Self-monitoring of oral anticoagulation: systematic review and metaanalysis of individual patient data. Lancet. 2012;379:322-334.

483. Crowther MA, Ageno W, Garcia D, Wang L, Witt DM, Clark NP, Blostein MD, Kahn SR, Vesely SK, Schulman S, Kovacs MJ, Rodger MA, Wells P, Anderson D, Ginsberg J, Selby R, Siragusa S, Silingardi M, Dowd MB, Kearon C. Oral vitamin K versus placebo to correct excessive anticoagulation in patients receiving warfarin: a randomized trial. Ann Intern Med. 2009;150:293-300.

484. Halvorsen S, Storey RF, Rocca B, Sibbing D, Ten Berg J, Grove EL, Weiss TW, Collet JP, Andreotti F, Gulba DC, Lip GYH, Husted S, Vilahur G, Morais J, Verheugt FWA, Lanas A, Al-Shahi Salman R, Steg PG, Huber K, ESC Working Group on Thrombosis. Management of antithrombotic therapy after bleeding in patients with coronary artery disease and/or atrial fibrillation: expert consensus paper of the European Society of Cardiology Working Group on Thrombosis. Eur Heart J. 2017;38:1455-1462.

485. Lubetsky A, Yonath H, Olchovsky D, Loebstein R, Halkin H, Ezra D. Comparison of oral vs intravenous phytonadione (vitamin K1) in patients with excessive anticoagulation: a prospective randomized controlled study. Arch Intern Med. 2003;163: 2469-2473.

486. Massel DR, Little SH. Antiplatelet and anticoagulation for patients with prosthetic heart valves. Cochrane Database Syst Rev 2013: CD003464.

487. Hansen ML, Sorensen R, Clausen MT, Fog-Petersen ML, Raunso J, Gadsboll N, Gislason GH, Folke F, Andersen SS, Schramm TK, Abildstrom SZ, Poulsen HE, Kober L, Torp-Pedersen C. Risk of bleeding with single, dual, or triple therapy with warfarin, aspirin, and clopidogrel in patients with atrial fibrillation. Arch Intern Med. 2010;170:1433-1441.

488. Douketis JD, Spyropoulos AC, Kaatz S, Becker RC, Caprini JA, Dunn AS, Garcia DA, Jacobson A, Jaffer AK, Kong DF, Schulman S, Turpie AG, Hasselblad V, Ortel TL, BRIDGE Investigators. Perioperative bridging anticoagulation in patients with atrial fibrillation. N Engl J Med. 2015;373:823-833.
489. Kristensen SD, Knuuti J, Saraste A, Anker S, Botker HE, Hert SD, Ford I, Gonzalez-Juanatey JR, Gorenek B, Heyndrickx GR, Hoeft A, Huber K, Iung B, Kjeldsen KP, Longrois D, Luscher TF, Pierard L, Pocock S, Price S, Roffi M, Sirnes PA, Sousa-Uva M, Voudris V, Funck-Brentano C, Authors/Task Force Members. 2014 ESC/ESA Guidelines on non-cardiac surgery: cardiovascular assessment and management: The Joint Task Force on non-cardiac surgery: cardiovascular assessment and management of the European Society of Cardiology (ESC) and the European Society of Anaesthesiology (ESA ). Eur Heart J. 2014;35:2383-2431.

490. Gellatly RM, Leet A, Brown KE. Fondaparinux: an effective bridging strategy in heparin-induced thrombocytopenia and mechanical circulatory support. J Heart Lung Transplant. 2014;33:118.

491. Brennan JM, Edwards FH, Zhao Y, O’Brien S, Booth ME, Dokholyan RS, Douglas PS, Peterson ED, DEcIDE AVR Research Team. Early anticoagulation of bioprosthetic aortic valves in older patients: results from the Society of Thoracic Surgeons Adult Cardiac Surgery National Database. J Am Coll Cardiol. 2012;60:971-977.

492. Merie C, Kober L, Skov Olsen P, Andersson C, Gislason G, Skov Jensen J, TorpPedersen C. Association of warfarin therapy duration after bioprosthetic aortic valve replacement with risk of mortality, thromboembolic complications, and bleeding. JAMA. 2012;308:2118-2125.

493. Christersson C, James SK, Lindhagen L, Ahlsson A, Friberg O, Jeppsson A, Stahle E. Comparison of warfarin versus antiplatelet therapy after surgical bioprosthetic aortic valve replacement. Heart. 2020;106:838-844.

494. Rafiq S, Steinbruchel DA, Lilleor NB, Moller CH, Lund JT, Thiis JJ, Kober L, Olsen PS. Antithrombotic therapy after bioprosthetic aortic valve implantation: warfarin versus aspirin, a randomized controlled trial. Thromb Res. 2017;150:104-110.

495. Maes F, Stabile E, Ussia GP, Tamburino C, Pucciarelli A, Masson JB, Marsal JR, Barbanti M, Cote M, Rodes-Cabau J. Meta-analysis comparing single versus dual antiplatelet therapy following transcatheter aortic valve implantation. Am J Cardiol. 2018;122:310-315.

496. Brouwer J, Nijenhuis VJ, Delewi R, Hermanides RS, Holvoet W, Dubois CLF, Frambach P, De Bruyne B, van Houwelingen GK, Van Der Heyden JAS, Tousek P, van der Kley F, Buysschaert I, Schotborgh CE, Ferdinande B, van der Harst P, Roosen J, Peper J, Thielen FWF, Veenstra L, Chan Pin Yin D, Swaans MJ, Rensing B, van 't Hof AWJ, Timmers L, Kelder JC, Stella PR, Baan J, Ten Berg JM. Aspirin with or without clopidogrel after transcatheter aortic-valve implantation. $N$ Engl $\mathrm{J} \mathrm{Med}$. 2020;383:1447-1457.

497. Dangas GD, Tijssen JGP, Wohrle J, Sondergaard L, Gilard M, Mollmann H, Makkar RR, Herrmann HC, Giustino G, Baldus S, De Backer O, Guimaraes AHC, Gullestad L, Kini A, von Lewinski D, Mack M, Moreno R, Schafer U, Seeger J, Tchetche D, Thomitzek K, Valgimigli M, Vranckx P, Welsh RC, Wildgoose P, Volkl AA, Zazula A, van Amsterdam RGM, Mehran R, Windecker S, GALILEO Investigators. A controlled trial of rivaroxaban after transcatheter aortic-valve replacement. N Engl J Med. 2020;382:120-129.

498. Pagnesi M, Moroni F, Beneduce A, Giannini F, Colombo A, Weisz G, Latib A. Thrombotic risk and antithrombotic strategies after transcatheter mitral valve replacement. JACC Cardiovasc Interv. 2019;12:2388-2401.

499. Guimaraes HP, Lopes RD, de Barros ESPGM, Liporace IL, Sampaio RO, Tarasoutchi F, Hoffmann-Filho CR, de Lemos Soares Patriota R, Leiria TLL, Lamprea D, Precoma DB, Atik FA, Silveira FS, Farias FR, Barreto DO, Almeida AP, Zilli AC, de Souza Neto JD, Cavalcante MA, Figueira F, Kojima FCS, Damiani L, Santos RHN, Valeis N, Campos VB, Saraiva JFK, Fonseca FH, Pinto IM, Magalhaes CC, Ferreira JFM, Alexander JH, Pavanello R, Cavalcanti AB, Berwanger O, RIVER Trial Investigators. Rivaroxaban in patients with atrial fibrillation and a bioprosthetic mitral valve. N Engl J Med. 2020;383:2117-2126.

500. Shim CY, Seo J, Kim YJ, Lee SH, De Caterina R, Lee S, Hong GR; Explore the Efficacy and Safety of Edoxaban in Patients after Heart Valve Repair or Bioprosthetic Valve Replacement (ENAVLE) study group. Efficacy and safety of edoxaban in patients early after surgical bioprosthetic valve implantation or valve repair: a randomized clinical trial. J Thorac Cardiovasc Surg 2021; doi: 10.1016/j.jtcvs.2021.01.127. 501. Nijenhuis VJ, Brouwer J, Delewi R, Hermanides RS, Holvoet W, Dubois CLF, Frambach P, De Bruyne B, van Houwelingen GK, Van Der Heyden JAS, Tousek P, van der Kley F, Buysschaert I, Schotborgh CE, Ferdinande B, van der Harst P, Roosen J, Peper J, Thielen FWF, Veenstra L, Chan Pin Yin D, Swaans MJ, Rensing B, van 't Hof AWJ, Timmers L, Kelder JC, Stella PR, Baan J, Ten Berg JM. Anticoagulation with or without clopidogrel after transcatheter aortic-valve implantation. $N$ Engl $J$ Med. 2020;382:1696-1707.

502. Jochheim D, Barbanti M, Capretti G, Stefanini GG, Hapfelmeier A, Zadrozny M, Baquet M, Fischer J, Theiss H, Todaro D, Chieffo A, Presbitero P, Colombo A, Massberg S, Tamburino C, Mehilli J. Oral anticoagulant type and outcomes after transcatheter aortic valve replacement. JACC Cardiovasc Interv. 2019;12:1566-1576.

503. van der Wall SJ, Olsthoorn JR, Heuts S, Klautz RJM, Tomsic A, Jansen EK, Vonk ABA, Sardari Nia P, Klok FA, Huisman MV. Antithrombotic therapy after mitral valve repair: VKA or aspirin? J Thromb Thrombolysis. 2018;46:473-481. 
504. Spyropoulos AC, Turpie AG, Dunn AS, Kaatz S, Douketis J, Jacobson A, Petersen H, REGIMEN Investigators. Perioperative bridging therapy with unfractionated heparin or low-molecular-weight heparin in patients with mechanical prosthetic heart valves on long-term oral anticoagulants (from the REGIMEN Registry). $A m J$ Cardiol. 2008;102:883-889.

505. Dewilde WJ, Oirbans T, Verheugt FW, Kelder JC, De Smet BJ, Herrman JP, Adriaenssens T, Vrolix M, Heestermans AA, Vis MM, Tijsen JG, van 't Hof AW, ten Berg JM, WOEST Study Investigators. Use of clopidogrel with or without aspirin in patients taking oral anticoagulant therapy and undergoing percutaneous coronary intervention: an open-label, randomised, controlled trial. Lancet. 2013;381:1107-1115.

506. Gibson CM, Mehran R, Bode C, Halperin J, Verheugt FW, Wildgoose P, Birmingham M, Ianus J, Burton P, van Eickels M, Korjian S, Daaboul Y, Lip GY, Cohen M, Husted S, Peterson ED, Fox KA. Prevention of bleeding in patients with atrial fibrillation undergoing PCI. N Engl J Med. 2016;375:2423-2434.

507. Lopes RD, Heizer G, Aronson R, Vora AN, Massaro T, Mehran R, Goodman SG, Windecker S, Darius H, Li J, Averkov O, Bahit MC, Berwanger O, Budaj A, Hijazi Z, Parkhomenko A, Sinnaeve P, Storey RF, Thiele H, Vinereanu D, Granger CB, Alexander JH, AUGUSTUS Investigators. Antithrombotic therapy after acute coronary syndrome or PCI in atrial fibrillation. N Engl J Med. 2019;380:1509-1524.

508. Fiedler KA, Maeng M, Mehilli J, Schulz-Schupke S, Byrne RA, Sibbing D, Hoppmann P, Schneider S, Fusaro M, Ott I, Kristensen SD, Ibrahim T, Massberg S, Schunkert H, Laugwitz KL, Kastrati A, Sarafoff N. Duration of triple therapy in patients requiring oral anticoagulation after drug-eluting stent implantation: the ISARTRIPLE Trial. J Am Coll Cardiol. 2015;65:1619-1629.

509. Vranckx P, Valgimigli M, Eckardt L, Tijssen J, Lewalter T, Gargiulo G, Batushkin V, Campo G, Lysak Z, Vakaliuk I, Milewski K, Laeis P, Reimitz PE, Smolnik R, Zierhut W, Goette A. Edoxaban-based versus vitamin K antagonist-based antithrombotic regimen after successful coronary stenting in patients with atrial fibrillation (ENTRUST-AF PCI): a randomised, open-label, phase 3b trial. Lancet. 2019; 394:1335-1343.

510. Lamberts M, Gislason GH, Lip GY, Lassen JF, Olesen JB, Mikkelsen AP, Sorensen R, Kober L, Torp-Pedersen C, Hansen ML. Antiplatelet therapy for stable coronary artery disease in atrial fibrillation patients taking an oral anticoagulant: a nationwide cohort study. Circulation. 2014;129:1577-1585.

511. Yasuda S, Kaikita K, Akao M, Ako J, Matoba T, Nakamura M, Miyauchi K, Hagiwara N, Kimura K, Hirayama A, Matsui K, Ogawa H, AFIRE Investigators. Antithrombotic therapy for atrial fibrillation with stable coronary disease. $N$ Engl J Med. 2019;381:1103-1113.

512. Collet JP, Thiele H, Barbato E, Barthelemy O, Bauersachs J, Bhatt DL, Dendale P, Dorobantu M, Edvardsen T, Folliguet T, Gale CP, Gilard M, Jobs A, Juni P, Lambrinou E, Lewis BS, Mehilli J, Meliga E, Merkely B, Mueller C, Roffi M, Rutten FH, Sibbing D, Siontis GCM, ESC Scientific Document Group. 2020 ESC Guidelines for the management of acute coronary syndromes in patients presenting without persistent ST-segment elevation. Eur Heart J. 2021;42:1289-1367.

513. Dewilde WJ, Janssen PW, Kelder JC, Verheugt FW, De Smet BJ, Adriaenssens T, Vrolix M, Brueren GB, Van Mieghem C, Cornelis K, Vos J, Breet NJ, ten Berg JM. Uninterrupted oral anticoagulation versus bridging in patients with long-term oral anticoagulation during percutaneous coronary intervention: subgroup analysis from the WOEST trial. EuroIntervention. 2015;11:381-390.

514. Lip GYH, Collet JP, Haude M, Byrne R, Chung EH, Fauchier L, Halvorsen S, Lau D, Lopez-Cabanillas N, Lettino M, Marin F, Obel I, Rubboli A, Storey RF, Valgimigli M, Huber K, ESC Scientific Document Group. 2018 Joint European consensus document on the management of antithrombotic therapy in atrial fibrillation patients presenting with acute coronary syndrome and/or undergoing percutaneous cardiovascular interventions: a joint consensus document of the European Heart Rhythm Association (EHRA), European Society of Cardiology Working Group on Thrombosis, European Association of Percutaneous Cardiovascular Interventions (EAPCI), and European Association of Acute Cardiac Care (ACCA) endorsed by the Heart Rhythm Society (HRS), Asia-Pacific Heart Rhythm Society (APHRS), Latin America Heart Rhythm Society (LAHRS), and Cardiac Arrhythmia Society of Southern Africa (CASSA). Europace. 2019;21:192-193.

515. Carnicelli AP, De Caterina R, Halperin JL, Renda G, Ruff CT, Trevisan M, Nordio F, Mercuri MF, Antman E, Giugliano RP, ENGAGE AF-TIMI 48 Investigators. Edoxaban for the prevention of thromboembolism in patients with atrial fibrillation and bioprosthetic valves. Circulation. 2017;135:1273-1275.

516. Philippart R, Brunet-Bernard A, Clementy N, Bourguignon T, Mirza A, Babuty D, Angoulvant D, Lip GY, Fauchier L. Prognostic value of $\mathrm{CHA}_{2} \mathrm{DS}_{2}$-VASc score in patients with 'non-valvular atrial fibrillation' and valvular heart disease: the Loire Valley Atrial Fibrillation Project. Eur Heart J. 2015;36:1822-1830.

517. Philippart R, Brunet-Bernard A, Clementy N, Bourguignon T, Mirza A, Angoulvant D, Babuty D, Lip GY, Fauchier L. Oral anticoagulation, stroke and thromboembolism in patients with atrial fibrillation and valve bioprosthesis. The Loire Valley Atrial Fibrillation Project. Thromb Haemost. 2016;115:1056-1063.
518. Siontis KC, Yao X, Gersh BJ, Noseworthy PA. Direct oral anticoagulants in patients with atrial fibrillation and valvular heart disease other than significant mitral stenosis and mechanical valves: a meta-analysis. Circulation. 2017;135:714-716.

519. Russo A, Grigioni F, Avierinos JF, Freeman WK, Suri R, Michelena H, Brown R, Sundt TM, Enriquez-Sarano M. Thromboembolic complications after surgical correction of mitral regurgitation incidence, predictors, and clinical implications. $J$ Am Coll Cardiol. 2008;51:1203-1211.

520. Butnaru A, Shaheen J, Tzivoni D, Tauber R, Bitran D, Silberman S. Diagnosis and treatment of early bioprosthetic malfunction in the mitral valve position due to thrombus formation. Am J Cardiol. 2013;112:1439-1444.

521. Rodes-Cabau J, Masson JB, Welsh RC, Garcia Del Blanco B, Pelletier M, Webb JG, Al-Qoofi F, Genereux P, Maluenda G, Thoenes M, Paradis JM, Chamandi C, Serra V, Dumont E, Cote M. Aspirin versus aspirin plus clopidogrel as antithrombotic treatment following transcatheter aortic valve replacement with a balloon-expandable valve: the ARTE (Aspirin Versus Aspirin + Clopidogrel Following Transcatheter Aortic Valve Implantation) randomized clinical trial. JACC Cardiovasc Interv. 2017; 10:1357-1365.

522. Dvir D, Bourguignon T, Otto CM, Hahn RT, Rosenhek R, Webb JG, Treede H, Sarano ME, Feldman T, Wijeysundera HC, Topilsky Y, Aupart M, Reardon MJ, Mackensen GB, Szeto WY, Kornowski R, Gammie JS, Yoganathan AP, Arbel Y, Borger MA, Simonato M, Reisman M, Makkar RR, Abizaid A, McCabe JM, Dahle G, Aldea GS, Leipsic J, Pibarot P, Moat NE, Mack MJ, Kappetein AP, Leon MB, VIVID Investigators. Standardized definition of structural valve degeneration for surgical and transcatheter bioprosthetic aortic valves. Circulation. 2018;137:388-399.

523. Tam DY, Dharma C, Rocha RV, Ouzounian M, Wijeysundera HC, Austin PC, Chikwe J, Gaudino M, Fremes SE. Transcatheter ViV versus redo surgical AVR for the management of failed biological prosthesis: early and late outcomes in a propensitymatched cohort. JACC Cardiovasc Interv. 2020;13:765-774.

524. Bleiziffer S, Simonato M, Webb JG, Rodes-Cabau J, Pibarot P, Kornowski R, Windecker S, Erlebach M, Duncan A, Seiffert M, Unbehaun A, Frerker C, Conzelmann L, Wijeysundera H, Kim WK, Montorfano M, Latib A, Tchetche D, Allali A, Abdel-Wahab M, Orvin K, Stortecky S, Nissen H, Holzamer A, Urena M, Testa L, Agrifoglio M, Whisenant B, Sathananthan J, Napodano M, Landi A, Fiorina C, Zittermann A, Veulemans V, Sinning JM, Saia F, Brecker S, Presbitero P, De Backer O, Sondergaard L, Bruschi G, Franco LN, Petronio AS, Barbanti M, Cerillo A, Spargias K, Schofer J, Cohen M, Munoz-Garcia A, Finkelstein A, Adam M, Serra V, Teles RC, Champagnac D, Iadanza A, Chodor P, Eggebrecht H, Welsh R, Caixeta A, Salizzoni S, Dager A, Auffret V, Cheema A, Ubben T, Ancona M, Rudolph T, Gummert J, Tseng E, Noble S, Bunc M, Roberts D, Kass M, Gupta A, Leon MB, Dvir D. Long-term outcomes after transcatheter aortic valve implantation in failed bioprosthetic valves. Eur Heart J. 2020;41:2731-2742

525. Hirji SA, Percy ED, Zogg CK, Malarczyk A, Harloff MT, Yazdchi F, Kaneko T. Comparison of in-hospital outcomes and readmissions for valve-in-valve transcathete aortic valve replacement vs. reoperative surgical aortic valve replacement: a contemporary assessment of real-world outcomes. Eur Heart J. 2020;41:2747-2755.

526. Barbanti M, Costa G, Picci A, Criscione E, Reddavid C, Valvo R, Todaro D Deste W, Condorelli A, Scalia M, Licciardello A, Politi G, De Luca G, Strazzieri O, Motta S, Garretto V, Veroux P, Giaquinta A, Giuffrida A, Sgroi C, Leon MB, Webb JG, Tamburino C. Coronary cannulation after transcatheter aortic valve replacement: the RE-ACCESS Study. JACC Cardiovasc Interv. 2020;13:2542-2555.

527. De Backer O, Landes U, Fuchs A, Yoon SH, Mathiassen ON, Sedaghat A, Kim WK, Pilgrim T, Buzzatti N, Ruile P, El Sabbagh A, Barbanti M, Fiorina C, Nombela-Franco L, Steinvil A, Finkelstein A, Montorfano M, Maurovich-Horvat P, Kofoed KF, Blanke P, Bunc M, Neumann FJ, Latib A, Windecker S, Sinning JM, Norgaard BL, Makkar R, Webb JG, Sondergaard L. Coronary access after TAVR-inTAVR as evaluated by multidetector computed tomography. JACC Cardiovasc Interv. 2020;13:2528-2538.

528. Jawitz OK, Gulack BC, Grau-Sepulveda MV, Matsouaka RA, Mack MJ, Holmes DR Jr., Carroll JD, Thourani VH, Brennan JM. Reoperation after transcatheter aortic valve replacement: an analysis of the Society of Thoracic Surgeons Database. JACC Cardiovasc Interv. 2020;13:1515-1525.

529. Dvir D, Webb JG, Bleiziffer S, Pasic M, Waksman R, Kodali S, Barbanti M, Latib A, Schaefer U, Rodes-Cabau J, Treede H, Piazza N, Hildick-Smith D, Himbert D, Walther T, Hengstenberg C, Nissen H, Bekeredjian R, Presbitero P, Ferrari E, Segev A de Weger A, Windecker S, Moat NE, Napodano M, Wilbring M, Cerillo AG, Brecker S, Tchetche D, Lefevre T, De Marco F, Fiorina C, Petronio AS, Teles RC, Testa L, Laborde JC, Leon MB, Kornowski R, Valve-in-Valve International Data Registry Investigators. Transcatheter aortic valve implantation in failed bioprosthetic surgical valves. JAMA. 2014;312:162-170.

530. Ye J, Cheung A, Yamashita M, Wood D, Peng D, Gao M, Thompson CR, Munt B, Moss RR, Blanke P, Leipsic J, Dvir D, Webb JG. Transcatheter aortic and mitral valvein-valve implantation for failed surgical bioprosthetic valves: an 8-year single-center experience. JACC Cardiovasc Interv. 2015;8:1735-1744. 
531. Simonato M, Whisenant B, Ribeiro HB, Webb JG, Kornowski R, Guerrero M, Wijeysundera H, Sondergaard L, De Backer O, Villablanca P, Rihal C, Eleid M, Kempfert J, Unbehaun A, Erlebach M, Casselman F, Adam M, Montorfano M, Ancona M, Saia F, Ubben T, Meincke F, Napodano M, Codner P, Schofer J, Pelletier M, Cheung A, Shuvy M, Palma JH, Gaia DF, Duncan A, Hildick-Smith D, Veulemans V, Sinning JM, Arbel Y, Testa L, de Weger A, Eltchaninoff H, Hemery T, Landes U, Tchetche D, Dumonteil N, Rodes-Cabau J, Kim WK, Spargias K, Kourkoveli P, BenYehuda O, Teles RC, Barbanti M, Fiorina C, Thukkani A, Mackensen GB, Jones N, Presbitero P, Petronio AS, Allali A, Champagnac D, Bleiziffer S, Rudolph T, Iadanza A, Salizzoni S, Agrifoglio M, Nombela-Franco L, Bonaros N, Kass M, Bruschi G, Amabile N, Chhatriwalla A, Messina A, Hirji SA, Andreas M, Welsh R, Schoels W, Hellig F, Windecker S, Stortecky S, Maisano F, Stone GW, Dvir D. Transcatheter mitral valve replacement after surgical repair or replacement: comprehensive midterm evaluation of valve-in-valve and valve-in-ring implantation from the VIVID Registry. Circulation. 2021;143:104-116.

532. Sengupta A, Yazdchi F, Alexis SL, Percy E, Premkumar A, Hirji S, Bapat VN, Bhatt DL, Kaneko T, Tang GHL. Reoperative mitral surgery versus transcatheter mitral valve replacement: a systematic review. J Am Heart Assoc. 2021;10: e019854.

533. Urena M, Vahanian A, Brochet E, Ducrocq G, Iung B, Himbert D. Current indications for transcatheter mitral valve replacement using transcatheter aortic valves: valve-in-valve, valve-in-ring, and valve-in-mitral annulus calcification. Circulation. 2021;143:178-196.

534. Little SH, Bapat V, Blanke P, Guerrero M, Rajagopal V, Siegel R. Imaging guidance for transcatheter mitral valve intervention on prosthetic valves, rings, and annular calcification. JACC Cardiovasc Imaging. 2021;14:22-40.

535. Fallon JM, DeSimone JP, Brennan JM, O’Brien S, Thibault DP, DiScipio AW, Pibarot P, Jacobs JP, Malenka DJ. The incidence and consequence of prosthesis-patient mismatch after surgical aortic valve replacement. Ann Thorac Surg. 2018;106:14-22. 536. Flameng W, Herregods MC, Vercalsteren M, Herijgers P, Bogaerts K, Meuris B. Prosthesis-patient mismatch predicts structural valve degeneration in bioprosthetic heart valves. Circulation. 2010;121:2123-2129.

537. Zorn GL 3rd, Little SH, Tadros P, Deeb GM, Gleason TG, Heiser J, Kleiman NS, Oh JK, Popma JJ, Adams D, Huang J, Reardon MJ. Prosthesis-patient mismatch in high-risk patients with severe aortic stenosis: a randomized trial of a self-expanding prosthesis. J Thorac Cardiovasc Surg. 2016;151:1014-1022, 1023. e1-3.

538. Head SJ, Mokhles MM, Osnabrugge RL, Pibarot P, Mack MJ, Takkenberg JJ, Bogers AJ, Kappetein AP. The impact of prosthesis-patient mismatch on long-term survival after aortic valve replacement: a systematic review and meta-analysis of 34 observational studies comprising 27186 patients with 133141 patient-years. Eur Heart J. 2012;33:1518-1529.

539. Sorajja P, Bae R, Lesser JA, Pedersen WA. Percutaneous repair of paravalvular prosthetic regurgitation: patient selection, techniques and outcomes. Heart. 2015; 101:665-673.

540. Ruiz CE, Hahn RT, Berrebi A, Borer JS, Cutlip DE, Fontana G, Gerosa G, Ibrahim R, Jelnin V, Jilaihawi H, Jolicoeur EM, Kliger C, Kronzon I, Leipsic J, Maisano F, Millan X, Nataf P, O'Gara PT, Pibarot P, Ramee SR, Rihal CS, RodesCabau J, Sorajja P, Suri R, Swain JA, Turi ZG, Tuzcu EM, Weissman NJ, Zamorano JL, Serruys PW, Leon MB, Paravalvular Leak Academic Research Consortium. Clinical trial principles and endpoint definitions for paravalvular leaks in surgical prosthesis. Eur Heart J. 2018;39:1224-1245.

541. Chakravarty T, Sondergaard L, Friedman J, De Backer O, Berman D, Kofoed KF, Jilaihawi H, Shiota T, Abramowitz Y, Jorgensen TH, Rami T, Israr S, Fontana G, de Knegt M, Fuchs A, Lyden P, Trento A, Bhatt DL, Leon MB, Makkar RR, Resolve, SAVORY Investigators. Subclinical leaflet thrombosis in surgical and transcatheter bioprosthetic aortic valves: an observational study. Lancet. 2017;389:2383-2392.

542. Karthikeyan G, Senguttuvan NB, Joseph J, Devasenapathy N, Bahl VK, Airan B. Urgent surgery compared with fibrinolytic therapy for the treatment of left-sided prosthetic heart valve thrombosis: a systematic review and meta-analysis of observational studies. Eur Heart J. 2013;34:1557-1566.

543. Laplace G, Lafitte S, Labeque JN, Perron JM, Baudet E, Deville C, Roques X, Roudaut R. Clinical significance of early thrombosis after prosthetic mitral valve replacement: a postoperative monocentric study of 680 patients. $J$ Am Coll Cardiol. 2004;43:1283-1290.

544. Petrescu I, Egbe AC, Ionescu F, Nkomo VT, Greason KL, Pislaru C, Pellikka PA, Connolly HM, Pislaru SV. Long-term outcomes of anticoagulation for bioprosthetic valve thrombosis. J Am Coll Cardiol. 2020;75:857-866.

545. Sellers SL, Turner CT, Sathananthan J, Cartlidge TRG, Sin F, Bouchareb R, Mooney J, Norgaard BL, Bax JJ, Bernatchez PN, Dweck MR, Granville DJ, Newby DE, Lauck S, Webb JG, Payne GW, Pibarot P, Blanke P, Seidman MA, Leipsic JA. Transcatheter aortic heart valves: histological analysis providing insight to leaflet thickening and structural valve degeneration. JACC Cardiovasc Imaging. 2019;12: $135-145$.

546. De Backer O, Dangas GD, Jilaihawi H, Leipsic JA, Terkelsen CJ, Makkar R, Kini AS, Veien KT, Abdel-Wahab M, Kim WK, Balan P, Van Mieghem N, Mathiassen ON, Jeger RV, Arnold M, Mehran R, Guimaraes AHC, Norgaard BL, Kofoed KF, Blanke P, Windecker S, Sondergaard L, GALILEO-4D Investigators. Reduced leaflet motion after transcatheter aortic-valve replacement. $N$ Engl J Med. 2020;382:130-139.

547. Alkhouli M, Rihal CS, Zack CJ, Eleid MF, Maor E, Sarraf M, Cabalka AK, Reeder GS, Hagler DJ, Maalouf JF, Nkomo VT, Schaff HV, Said SM. Transcatheter and surgical management of mitral paravalvular leak: long-term outcomes. JACC Cardiovasc Interv. 2017;10:1946-1956.

548. Dakik HA, Chehab O, Eldirani M, Sbeity E, Karam C, Abou Hassan O, Msheik M, Hassan H, Msheik A, Kaspar C, Makki M, Tamim H. A new index for pre-operative cardiovascular evaluation. J Am Coll Cardiol. 2019;73:3067-3078.

549. Tashiro T, Pislaru SV, Blustin JM, Nkomo VT, Abel MD, Scott CG, Pellikka PA. Perioperative risk of major non-cardiac surgery in patients with severe aortic stenosis: a reappraisal in contemporary practice. Eur Heart J. 2014;35:2372-2381.

550. Eugene M, Urena M, Abtan J, Carrasco JL, Ghodbane W, Nataf P, Vahanian A, Himbert D. Effectiveness of rescue percutaneous balloon aortic valvuloplasty in patients with severe aortic stenosis and acute heart failure. Am J Cardiol. 2018;121: 746-750.

551. Kolte D, Khera S, Vemulapalli S, Dai D, Heo S, Goldsweig AM, Aronow HD, Elmariah S, Inglessis I, Palacios IF, Thourani VH, Sharaf BL, Gordon PC, Abbott JD. Outcomes following urgent/emergent transcatheter aortic valve replacement: insights from the STS/ACC TVT Registry. JACC Cardiovasc Interv. 2018;11:1175-1185.

552. Calleja AM, Dommaraju S, Gaddam R, Cha S, Khandheria BK, Chaliki HP. Cardiac risk in patients aged $>75$ years with asymptomatic, severe aortic stenosis undergoing noncardiac surgery. Am J Cardiol. 2010;105:1159-1163.

553. Tarantini G, Nai Fovino L, Tellaroli P, Fabris T, Iliceto S. Asymptomatic severe aortic stenosis and noncardiac surgery. Am J Cardiol. 2016;117:486-488.

554. Regitz-Zagrosek V, Roos-Hesselink JW, Bauersachs J, Blomstrom-Lundqvist C, Cifkova R, De Bonis M, Iung B, Johnson MR, Kintscher U, Kranke P, Lang IM, Morais J, Pieper PG, Presbitero P, Price S, Rosano GMC, Seeland U, Simoncini T, Swan L, Warnes CA, ESC Scientific Document Group. 2018 ESC Guidelines for the management of cardiovascular diseases during pregnancy. Eur Heart J. 2018; 39:3165-3241.

555. Roos-Hesselink J, Baris L, Johnson M, De Backer J, Otto C, Marelli A, Jondeau G, Budts W, Grewal J, Sliwa K, Parsonage W, Maggioni AP, van Hagen I, Vahanian A, Tavazzi L, Elkayam U, Boersma E, Hall R. Pregnancy outcomes in women with cardiovascular disease: evolving trends over 10 years in the ESC Registry Of Pregnancy And Cardiac disease (ROPAC). Eur Heart J. 2019;40:3848-3855.

556. van Hagen IM, Thorne SA, Taha N, Youssef G, Elnagar A, Gabriel H, ElRakshy Y, Iung B, Johnson MR, Hall R, Roos-Hesselink JW, ROPAC Investigators and EORP Team. Pregnancy outcomes in women with rheumatic mitral valve disease: results from the registry of pregnancy and cardiac disease. Circulation. 2018;137:806-816.

557. Orwat S, Diller GP, van Hagen IM, Schmidt R, Tobler D, Greutmann M, Jonkaitiene R, Elnagar A, Johnson MR, Hall R, Roos-Hesselink JW, Baumgartner H, ROPAC Investigators. Risk of pregnancy in moderate and severe aortic stenosis: from the Multinational ROPAC Registry. J Am Coll Cardiol. 2016;68:1727-1737.

558. Meijboom LJ, Vos FE, Timmermans J, Boers GH, Zwinderman AH, Mulder BJ. Pregnancy and aortic root growth in the Marfan syndrome: a prospective study. Eur Heart J. 2005;26:914-920.

559. McKellar SH, MacDonald RJ, Michelena HI, Connolly HM, Sundt TM 3rd. Frequency of cardiovascular events in women with a congenitally bicuspid aortic valve in a single community and effect of pregnancy on events. Am J Cardiol. 2011;107: 96-99.

560. Fuchs A, Urena M, Chong-Nguyen C, Kikoine J, Brochet E, Abtan J, Fischer Q, Ducrocq G, Vahanian A, Iung B, Himbert D. Valve-in-valve and valve-in-ring transcatheter mitral valve implantation in young women contemplating pregnancy. Circ Cardiovasc Interv. 2020;13: e009579.

561. van Hagen IM, Roos-Hesselink JW, Ruys TP, Merz WM, Goland S, Gabriel H, Lelonek M, Trojnarska O, Al Mahmeed WA, Balint HO, Ashour Z, Baumgartner H, Boersma E, Johnson MR, Hall R; ROPAC Investigators and the EURObservational Research Programme (EORP) Team. Pregnancy in women with a mechanical heart valve: data of the European Society of Cardiology Registry of Pregnancy and Cardiac Disease (ROPAC). Circulation. 2015;132:132-142.

562. Elassy SM, Elmidany AA, Elbawab HY. Urgent cardiac surgery during pregnancy: a continuous challenge. Ann Thorac Surg. 2014;97:1624-1629. 


\section{ESC/EACTS Guidelines for the}

\section{management of valvular heart disease} Supplementary data

\section{Developed by the Task Force for the management of valvular heart disease of the European Society of Cardiology (ESC) and the European Association for Cardio-Thoracic Surgery (EACTS) Authors/Task Force Members: Alec Vahanian (1) * (ESC Chairperson) (France), Friedhelm Beyersdorf*1 (EACTS Chairperson) (Germany), Fabien Praz} (ESC Task Force Coordinator) (Switzerland), Milan Milojevic ${ }^{1}$ (EACTS Task Force Coordinator) (Serbia), Stephan Baldus (Germany), Johann Bauersachs (Germany), Davide Capodanno (Italy), Lenard Conradi ${ }^{1}$ (Germany), Michele De Bonis ${ }^{1}$ (Italy), Ruggero De Paulis ${ }^{1}$ (Italy), Victoria Delgado (Netherlands), Nick Freemantle ${ }^{1}$ (United Kingdom), Martine Gilard (France), Kristina H. Haugaa (Norway), Anders Jeppsson ${ }^{1}$ (Sweden), Peter Jüni (Canada), Luc Pierard (Belgium), Bernard D. Prendergast (United Kingdom), J. Rafael Sádaba ${ }^{1}$ (Spain), Christophe Tribouilloy (France), Wojtek Wojakowski (Poland), ESC/EACTS Scientific Document Group

\footnotetext{
* Corresponding authors: Alec Vahanian, UFR Medecine, Université de Paris, site Bichat, 16 rue Huchard, 75018 Paris, France; and LVTS INSERM U1148, GH Bichat, 46, rue Henri Huchard, 75018 Paris, France. Tel: + 3366315 56 68, E-mail: Alec.vahanian@gmail.com; Friedhelm Beyersdorf, Department of Cardiovascular Surgery, University Heart Center, University Hospital Freiburg, Germany; and Medical Faculty of the Albert-Ludwigs-University, Freiburg, Germany, Hugstetterstr. 55, D-79106 Freiburg, Germany. Tel: +49761270 28180. E-mail: friedhelm.beyersdorf@uniklinik-freiburg.de
}

Author/Task Force Member affiliations: listed in Author information in the full text.

ESC Clinical Practice Guidelines Committee (CPG): listed in the Appendix in the full text.

EACTS Council: listed in the Appendix in the full text.

${ }^{1}$ Representing the European Association for Cardio-Thoracic Surgery (EACTS)

ESC subspecialty communities having participated in the development of this document:

Associations: Association for Acute CardioVascular Care (ACVC), European Association of Cardiovascular Imaging (EACVI), European Association of Percutaneous Cardiovascular Interventions (EAPCl), European Heart Rhythm Association (EHRA), Heart Failure Association (HFA).

Councils: Council on Valvular Heart Disease.

Working Groups: Cardiovascular Surgery, Thrombosis.

Patient Forum

The content of these European Society of Cardiology (ESC) / European Association for Cardio-Thoracic Surgery (EACTS) Guidelines has been published for personal and educational use only. No commercial use is authorized. No part of the ESC/EACTS Guidelines may be translated or reproduced in any form without written permission from the ESC and the EACTS. Permission can be obtained upon submission of a written request to Oxford University Press, the publisher of the European Heart Journal and the party authorized to handle such permissions on behalf of the ESC (journals.permissions@oup.com).

Disclaimer

The ESC/EACTS Guidelines represent the views of the ESC and the EACTS and were produced after careful consideration of the scientific and medical knowledge and the evidence available at the time of their publication. The ESC and the EACTS is/are not responsible in the event of any contradiction, discrepancy and/or ambiguity between the ESC/EACTS Guidelines and any other official recommendations or guidelines issued by the relevant public health authorities, in particular in relation to good use of healthcare or therapeutic strategies. Health professionals are encouraged to take the ESC/EACTS Guidelines fully into account when exercising their clinical judgment, as well as in the determination and the implementation of preventive, diagnostic or therapeutic medical strategies; however, the ESC/EACTS Guidelines do not override, in any way whatsoever, the individual responsibility of health professionals to make appropriate and accurate decisions in consideration of each patient's health condition and in consultation with that patient and, where appropriate and/or necessary, the patient's caregiver. Nor do the ESC/EACTS Guidelines exempt health professionals from taking into full and careful consideration the relevant official updated recommendations or guidelines issued by the competent public health authorities, in order to manage each patient's case in light of the scientifically accepted data pursuant to their respective ethical and professional obligations. It is also the health professional's responsibility to verify the applicable rules and regulations relating to drugs and medical devices at the time of prescription.

This article has been co-published with permission in the European Heart Journal and European Journal of Cardio-Thoracic Surgery. $\odot$ the European Society of Cardiology and the European Association for Cardio-Thoracic Surgery 2021. All rights reserved. The articles are identical except for minor stylistic and spelling differences in keeping with each journal's style. Either citation can be used when citing this article. For permissions, please email journals.permissions@oup.com. 


\section{Document Reviewers: Franz-Josef Neumann (ESC Review Coordinator) (Germany), Patrick Myers ${ }^{1}$ (EACTS Review Coordinator) (Switzerland), Magdy Abdelhamid (Egypt), Stephan Achenbach (Germany), Riccardo Asteggiano (Italy), Fabio Barili ${ }^{1}$ (Italy), Michael A. Borger (Germany), Thierry Carrel ${ }^{1}$ (Switzerland), Jean-Philippe Collet (France), Dan Foldager (Denmark), Gilbert Habib (France), Christian Hassager (Denmark), Alar Irs ${ }^{1}$ (Estonia), Bernard lung (France), Marjan Jahangiri ${ }^{1}$ (United Kingdom), Hugo A. Katus (Germany), Konstantinos C. Koskinas (Switzerland), Steffen Massberg (Germany), Christian E. Mueller (Switzerland), Jens Cosedis Nielsen (Denmark), Philippe Pibarot (Canada), Amina Rakisheva (Kazakhstan), Marco Roffi (Switzerland), Andrea Rubboli (Italy), Evgeny Shlyakhto (Russia), Matthias Siepe' (Germany), Marta Sitges (Spain), Lars Sondergaard (Denmark), Miguel Sousa-Uva' (Portugal), Guiseppe Tarantini (Italy), Jose Luis Zamorano (Spain)}

All experts involved in the development of these guidelines have submitted declarations of interest. These have been compiled in a report and published in a supplementary document simultaneously to the guidelines. The report is also available on the ESC website www.escardio.org/guidelines

\section{Table of Contents}

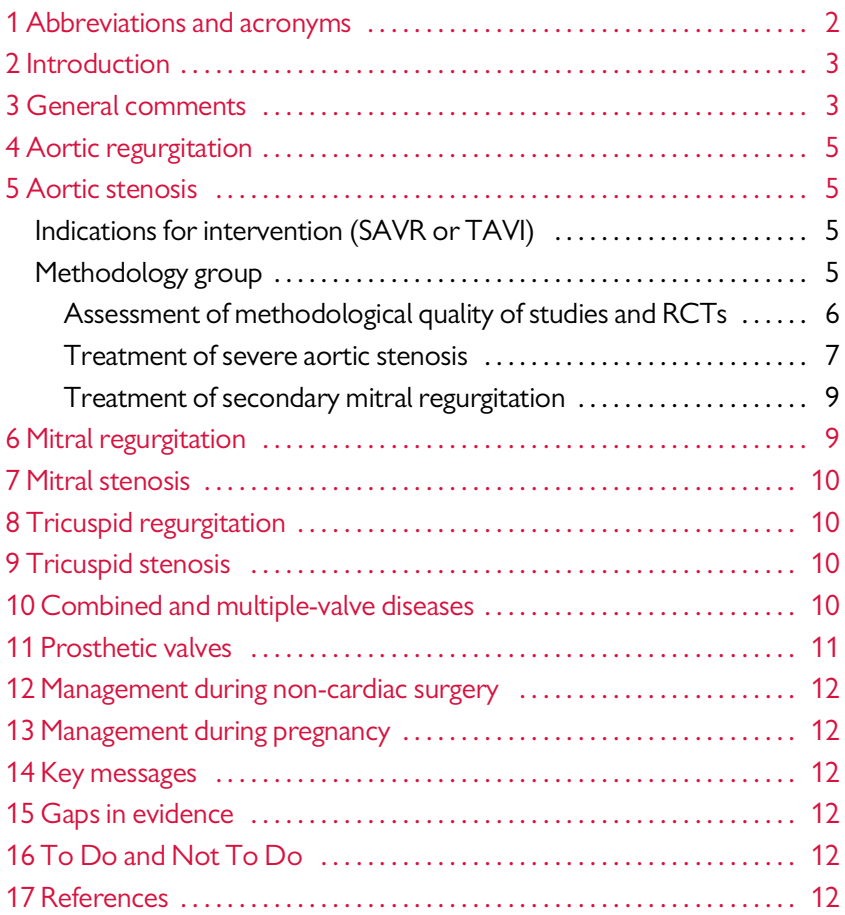

\section{List of Tables}

Supplementary Table 1 Cardiovascular and non-cardiovascular factors linked with transcatheter aortic valve implantation-related futility featured within the PARTNER and FRANCE 2 transcatheter aortic valve implantation-risk score models .................. 3

Supplementary Table 2 Katz Index of Independence in Activities

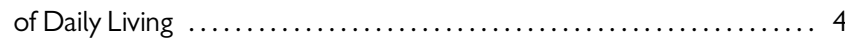

Supplementary Table 3 Essential frailty toolset in older adults undergoing aortic valve replacement

Supplementary Table 4 Medical comorbidities and factors predicting poorer outcomes post transcatheter aortic valve implantation ...................................... 4

Supplementary Table 5 Integrated approach for estimating transcatheter aortic valve implantation-specific risk and futility ...... 5 Supplementary Table 6 Risk-of-bias judgments for all evaluated

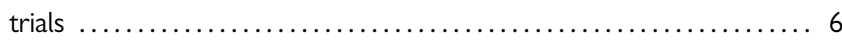

Supplementary Table 7 Main inclusion/exclusion criteria suggesting an increased chance of responding to TEER in patients with SMR

Supplementary Table 8 Echocardiographic scores used for assessing the feasibility of percutaneous mitral commissurotomy: Wilkins score, Cormier score, and Echo score 'Revisited'

\section{List of Figures}

Supplementary Figure 1 Criteria for patients selection for MitraClip procedure ................................ 5 Supplementary Figure 2 Peri- and post-procedural management of antithrombotic therapy in patients with indication to OAC

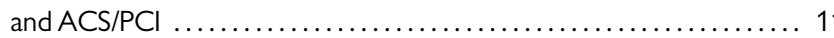
Supplementary Figure 3 Management of OAC in patients with an indication for preoperative bridging 


\section{Abbreviations and acronyms}

6MWT 6-minute walk test

ACEI Angiotensin-converting enzyme inhibitor

ACS Acute coronary syndrome

AF Atrial fibrillation

ARB Angiotensin receptor blocker

ARNI Angiotensin receptor-neprilysin inhibitors

ASA Acetylsalicylic acid

BMI Body mass index

CABG Coronary artery bypass grafting

$\mathrm{Cl} \quad$ Confidence interval

DLCO Diffusing Capacity of lung for carbon monoxide

DMR Diastolic mitral regurgitation

EACTS European Association for Cardio-Thoracic Surgery

EFT Essential Frailty Toolset

ESC European Society of Cardiology

FEV1 Forced expiratory volume in 1 second

FMR Functional mitral regurgitation

GDMT Guideline-directed medical therapy

Heart Tx Heart transplantation

INR International normalized ratio

LAA Left atrial appendage

LMWH Low-molecular-weight heparin

LTFU Lost to follow-up

LV Left ventricle/left ventricular

LVAD Left ventricular assist devices

LVEF Left ventricular ejection fraction

MR Mitral regurgitation

MVA Mitral valve area

NOAC Non-vitamin $\mathrm{K}$ antagonist oral anticoagulant

NYHA New York Heart Association

OAC Oral anticoagulation

PAP Pulmonary artery pressure

$\mathrm{PCl} \quad$ Percutaneous coronary intervention

$\mathrm{PH} \quad$ Pulmonary hypertension

QOL Quality of life

RCT Randomized controlled trial

RV Right ventricle/right ventricular

SAVR Surgical aortic valve replacement

SMR Secondary mitral regurgitation

SPAP Systolic pulmonary arterial pressure

TAVI Transcatheter aortic valve implantation

TAVR Transcatheter aortic valve replacement

TEER Transcatheter edge-to-edge repair

TMVR Transcatheter mitral valve repair

TTE Transthoracic echocardiography

UFH Unfractionated heparin

VHD Valvular heart disease

VKA Vitamin $\mathrm{K}$ antagonist

\section{Introduction}

There is no supplementary material for this section.

\section{General comments}

\section{Supplementary Table I Cardiovascular and non- cardiovascular factors linked with transcatheter aortic valve implantation-related futility featured within the PARTNER and FRANCE 2 transcatheter aortic valve implantation-risk score models}

\begin{tabular}{|c|c|c|}
\hline & PARTNER risk score & FRANCE 2 risk score \\
\hline \multirow{6}{*}{$\begin{array}{l}\text { Non- } \\
\text { cardiovascular } \\
\text { factors }\end{array}$} & & Age $\geq 90$ years \\
\hline & & $\mathrm{BMI}<30 \mathrm{~kg} / \mathrm{m}^{2}$ \\
\hline & Higher serum creatinine & Dialysis \\
\hline & $\begin{array}{l}\text { Oxygen-dependent } \\
\text { chronic lung disease }\end{array}$ & Respiratory insufficiency \\
\hline & $\begin{array}{l}\text { Lower mini-mental status } \\
\text { exam }\end{array}$ & \\
\hline & & Non-transfemoral access \\
\hline \multirow{5}{*}{$\begin{array}{l}\text { Cardiovascular } \\
\text { factors }\end{array}$} & Major arrhythmia (AF) & NYHA Class IV \\
\hline & $\begin{array}{l}\text { Lower mean trans-aortic } \\
\text { gradient }\end{array}$ & $\begin{array}{l}\text { Critical haemodynamic } \\
\text { state } \geq 2 \text { pulmonary } \\
\text { oedemas/year }\end{array}$ \\
\hline & Lower 6MWT distance & Pulmonary hypertension \\
\hline & \multicolumn{2}{|c|}{ High-prohibitive risk } \\
\hline & $\begin{array}{l}>50 \% \text { mortality or lack of } \\
\text { quality-of-life improve- } \\
\text { ment at } 6 \text { months }\end{array}$ & $>15 \% 30$-day mortality \\
\hline
\end{tabular}

$\mathrm{AF}=$ atrial fibrillation; $6 \mathrm{MWT}=6$-minute walk test; $\mathrm{BMI}=$ body mass index NYHA = New York Heart Association.

Reproduced from Puri R et al., TAVI or no TAVI: identifying patients unlikely to benefit from transcatheter aortic valve implantation. Eur Heart J 2016;37:2217-2225, by permission of Oxford University Press on behalf of the European Society of Cardiology. ${ }^{1}$ 


\section{Supplementary Table 2 Katz Index of Independence in Activities of Daily Living}

\begin{tabular}{|c|c|c|c|}
\hline \multicolumn{4}{|c|}{ Patient's name and last name: } \\
\hline & $\begin{array}{l}\text { Activities } \\
\text { point } \\
(1 \text { or } 0)\end{array}$ & $\begin{array}{l}\text { Independence } \\
\text { (1 point) }\end{array}$ & $\begin{array}{l}\text { Dependence } \\
\text { (0 points) }\end{array}$ \\
\hline Bathing & & $\begin{array}{l}\text { Bathes himself/her- } \\
\text { self completely or } \\
\text { needs partial help } \\
\text { while cleaning her } \\
\text { back or genital } \\
\text { region }\end{array}$ & $\begin{array}{l}\text { Needs help while } \\
\text { getting in or out of } \\
\text { the tub or shower, } \\
\text { and while cleaning } \\
\text { more than one part } \\
\text { of the body }\end{array}$ \\
\hline Dressing & & $\begin{array}{l}\text { Dress himself/herself } \\
\text { completely. May } \\
\text { sometimes need } \\
\text { help when tying } \\
\text { shoes }\end{array}$ & $\begin{array}{l}\text { Completely needs } \\
\text { help while dressing }\end{array}$ \\
\hline Toileting & & $\begin{array}{l}\text { Goes to toilet, gets } \\
\text { on and off, clean } \\
\text { genital area and puts } \\
\text { on his/her clothing } \\
\text { without help }\end{array}$ & $\begin{array}{l}\text { Needs help while } \\
\text { going to the toilet, } \\
\text { cleaning self, and } \\
\text { dressing }\end{array}$ \\
\hline Mobilization & & $\begin{array}{l}\text { Gets up from the } \\
\text { bed and chair on his/ } \\
\text { her own. May need } \\
\text { help for carrying } \\
\text { loads }\end{array}$ & $\begin{array}{l}\text { Needs help while } \\
\text { getting up from bed } \\
\text { to the chair }\end{array}$ \\
\hline Incontinence & & $\begin{array}{l}\text { May control himself/ } \\
\text { herself while urinat- } \\
\text { ing and defecating }\end{array}$ & $\begin{array}{l}\text { Partially or com- } \\
\text { pletely incontinent } \\
\text { of bowel or bladder }\end{array}$ \\
\hline Feeding & & $\begin{array}{l}\text { Gets foods from } \\
\text { plate into mouth } \\
\text { without help. May } \\
\text { need help while pre- } \\
\text { paring food }\end{array}$ & $\begin{array}{l}\text { Needs complete or } \\
\text { partial help with } \\
\text { feeding or requires } \\
\text { parenteral nutrition }\end{array}$ \\
\hline
\end{tabular}

Adapted from Katz S., Assessing self-maintenance: activities of daily living, mobility, and instrumental activities of daily living. J Am Geriatr Soc 1983;31:721-727. Copyright (1983), with permission from Wiley. ${ }^{2}$
Supplementary Table 3 Essential frailty toolset in older adults undergoing aortic valve replacement

\begin{tabular}{|c|c|c|}
\hline \multicolumn{3}{|c|}{ EFT Score } \\
\hline \multicolumn{2}{|l|}{ Five Chair rises $<15$ seconds } & 0 Points \\
\hline \multicolumn{2}{|l|}{ Five Chair rises $\geq 15$ seconds } & 1 Point \\
\hline \multicolumn{2}{|l|}{ Unable to complete } & 2 Points \\
\hline \multicolumn{2}{|l|}{ No cognitive impairment } & 0 Points \\
\hline \multicolumn{2}{|l|}{ Cognitive impairment } & 1 Point \\
\hline \multicolumn{2}{|l|}{ Haemoglobin $\geq 13.0 \mathrm{~g} / \mathrm{dL} \hat{~} \widehat{A}$} & 0 Points \\
\hline $\begin{array}{l}\text { Haemoglobin }<13.0 \mathrm{~g} / \mathrm{dL}{ }^{\star} \\
\text { Haemoglobin }<12.0 \mathrm{~g} / \mathrm{dL} \text { q }\end{array}$ & \multicolumn{2}{|c|}{ Haemoglobin $<12.0 \mathrm{~g} / \mathrm{dL}$ ? } \\
\hline \multicolumn{2}{|l|}{ Serum albumin $\geq 3.5 \mathrm{~g} / \mathrm{dL}$} & 0 Points \\
\hline \multicolumn{2}{|l|}{ Serum albumin $<3.5 \mathrm{~g} / \mathrm{dL}$} & 1 Point \\
\hline \multicolumn{3}{|l|}{ TOTAL SCORE: } \\
\hline \multicolumn{3}{|c|}{ Score interpretation } \\
\hline \multirow[t]{2}{*}{ EFT Score } & \multicolumn{2}{|c|}{ 1-Year Mortality } \\
\hline & TAVI & SAVR \\
\hline $0-1$ & $6 \%$ & $3 \%$ \\
\hline 2 & $15 \%$ & $7 \%$ \\
\hline 3 & $28 \%$ & $16 \%$ \\
\hline 4 & $30 \%$ & $38 \%$ \\
\hline 5 & $65 \%$ & $50 \%$ \\
\hline $\begin{array}{l}\text { EFT=Essential Frailty Toolset; } \\
\text { TAVI = transcatheter aortic valve } \\
\text { Reprinted from Afilalo J et al. } \\
\text { replacement: the FRAILTY-AVR } \\
\text { Copyright (2017), with permissi } \\
\text { Foundation. }\end{array}$ & $\begin{array}{l}\mathrm{VR}=\text { surgi } \\
\text { ntation. } \\
\text { in older } \\
\text { dy. } \int \mathrm{Am} \\
\text { om the }\end{array}$ & $\begin{array}{l}\text { acement; } \\
\text { tic valve } \\
89-700 . \\
\text { ardiology }\end{array}$ \\
\hline
\end{tabular}

Supplementary Table 4 Medical comorbidities and factors predicting poorer outcomes post transcatheter aortic valve implantation

\begin{tabular}{|l|l|}
\hline Medical comorbidity & $\begin{array}{l}\text { Factors specifically associated } \\
\text { with futility }\end{array}$ \\
\hline Chronic lung disease & $\begin{array}{l}6 \mathrm{MWT}<150 \mathrm{~m} \\
\text { Oxygen-dependency }\end{array}$ \\
\hline $\begin{array}{l}\text { Advanced chronic } \\
\text { kidney disease }\end{array}$ & $\begin{array}{l}\text { Atrial fibrillation } \\
\text { Dialysis dependence }\end{array}$ \\
\hline Frailty & $>2$ frailty indices (Katz activities of daily \\
& living + mobility status
\end{tabular}

$6 \mathrm{MWT}=6$-minute walk test; $\mathrm{LV}=$ left ventricle/left ventricular; $\mathrm{LVEF}=$ left ventricular ejection fraction; $M R$ = mitral regurgitation; $P A P=$ pulmonary artery pressure; $\mathrm{PH}=$ pulmonary hypertension; $\mathrm{TAVI}=$ transcatheter aortic valve implantation

${ }^{a}$ Time taken to walk $5 \mathrm{~m}$ is $>6$ seconds. Katz indices are: independence in feeding, bathing, dressing, transferring, toileting, urinary incontinence.

bMeasured invasively. Combined $\mathrm{PH}$ defined as post-capillary $\mathrm{PH}$ (measured by LV end-diastolic pressure $>15 \mathrm{mmHg}$ ) with a diastolic PAP $\geq 7 \mathrm{mmHg}$ than LV end-diastolic pressure.

Reproduced from Puri R et al., TAVI or no TAVI: identifying patients unlikely to benefit from transcatheter aortic valve implantation. Eur Heart J 2016;37:2217-2225, by permission of Oxford University Press on behalf of the European Society of Cardiology. ${ }^{1}$ 


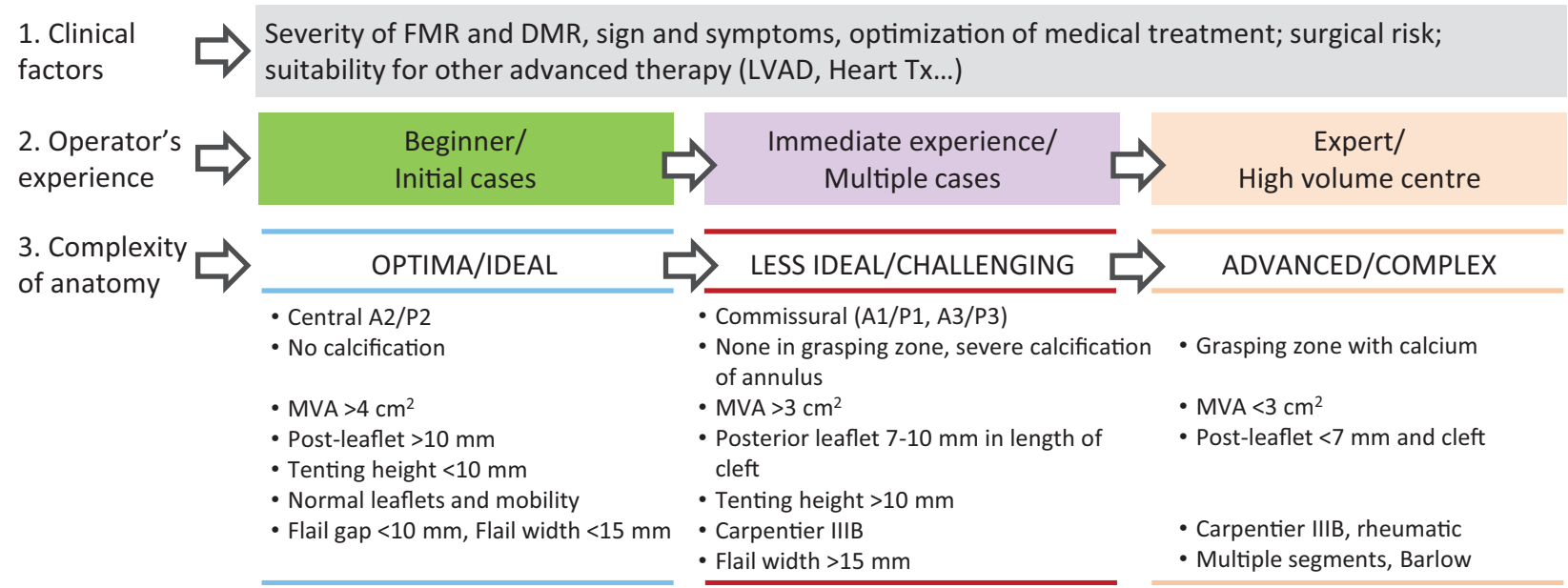

Supplementary Figure I Criteria for patients selection for MitraClip procedure. DMR=Degenerative mitral regurgitation; FMR = functional mitral regurgitation; Heart $T x=$ heart transplantation; $L V A D=$ left ventricular assist devices; MVA = mitral valve area. Reproduced from Gavazzoni $M$ et al., Conceiving MitraClip as a tool: percutaneous edge-to-edge repair in complex mitral valve anatomies. Eur Heart J Cardiovasc Imaging 2020;21:1059-1067, by permission of Oxford University Press on behalf of the European Society of Cardiology. ${ }^{4}$

Supplementary Table 5 Integrated approach for estimating transcatheter aortic valve implantation-specific risk and futility

\begin{tabular}{|c|c|c|c|c|}
\hline Criteria & Low risk & Intermediate risk & High risk & Prohibitive risk \\
\hline $\begin{array}{l}\text { PARTNER TAVI score }{ }^{a} \\
\text { OR } \\
\text { FRANCE } 2 \text { TAVI score }\end{array}$ & $\begin{array}{l}<25 \% \text { risk of mortality or lack } \\
\text { of QOL improvement at } 6 \\
\text { months } \\
\text { Risk score: } 0 \text { (30-day mortal- } \\
\text { ity risk }<5 \%)\end{array}$ & $\begin{array}{l}25-50 \% \text { risk of mortality or } \\
\text { lack of QOL improvement } \\
\text { at } 6 \text { months } \\
\text { Risk score: } 1-5(30 \text {-day mor- } \\
\text { tality risk } 5-15 \%)\end{array}$ & $\begin{array}{l}>50 \% \text { risk of mortality or lack } \\
\text { of QOL improvement at } 6 \\
\text { months } \\
\text { Risk score: } 6-7 \text { (30-day mor- } \\
\text { tality risk } 15-25 \%)\end{array}$ & $\begin{array}{l}\text { Risk score } \geq 8 \text { ( } 30 \text {-day } \\
\text { mortality risk }>25 \% \text { ) }\end{array}$ \\
\hline Frailty $^{\mathrm{b}}$ & None & 1 index & $\geq 2$ indices & $\geq 4$ indices \\
\hline $\begin{array}{l}\text { Specific major organ system } \\
\text { compromise not to be } \\
\text { improved post-TAVI }^{\mathrm{c}}\end{array}$ & None & 1 organ system & 2 organ systems & $\geq 3$ organ systems \\
\hline
\end{tabular}

$\mathrm{DLCO}=$ diffusing capacity of lung carbon monoxide; FEV1 = forced expiratory volume in 1 second; INR = international normalized ratio; LV= left ventricle/left ventricular; $\mathrm{QOL}=$ quality of life; $\mathrm{RV}=$ right ventricle/right ventricular; $\mathrm{TAVI}=$ transcatheter aortic valve implantation; VKA = vitamin $\mathrm{K}$ antagonist.

ahttp://h-outcomes.com/tavi-risk-calculator/.

${ }^{\mathrm{b}}$ Frailty based on Katz Index (independence in feeding, bathing, dressing, transferring, toileting, and urinary incontinence) and independence in ambulation (walk $5 \mathrm{~m}$ in $<6$ seconds). 'Examples of major organ system compromise: Cardiac (severe LV systolic or diastolic dysfunction or RV dysfunction, and fixed pulmonary hypertension); chronic kidney disease stage 3 or worse; pulmonary dysfunction with FEV1 $<50 \%$ or DLCO $<50 \%$ of predicted; central nervous system dysfunction (dementia, Alzheimer's disease, Parkinson's disease, and cerebrovascular accident with persistent physical limitation); gastrointestinal dysfunction (Crohn's disease, ulcerative colitis, nutritional impairment, or serum albumin <3.0); cancer (active malignancy); and liver (any history of cirrhosis, variceal bleeding, or elevated INR in the absence of VKA therapy).

Reproduced from Puri R et al., TAVI or no TAVI: identifying patients unlikely to benefit from transcatheter aortic valve implantation. Eur Heart J 2016;37:2217-2225, by permission of Oxford University Press on behalf of the European Society of Cardiology. ${ }^{1}$

\section{Aortic regurgitation}

There is no supplementary material for this section.

\section{Aortic stenosis}

\section{Indications for intervention (SAVR or TAVI)}

Assessment of methodological quality of studies and randomized controlled trials (RCTs) by the Methodology Group of the 2021 European Society of Cardiology (ESC)/ European Association for Cardio-Thoracic Surgery (EACTS) Guidelines for the management of valvular heart disease (VHD).

\section{Methodology group}

In preparation of the 2021 VHD Guidelines, a methodology group has been created for the first time to assist the Task Force for the collection and interpretation of the evidence supporting specific recommendations. The group was constituted by 2 ESC delegates and 2 EACTS delegates who were also members of the Task Force. Although the main activity of the group concerned the chapter on aortic stenosis and secondary mitral regurgitation (SMR), it was not limited to these two domains. The group was at disposal upon request of the Task Force members to resolve other specific methodological issues.

\section{Assessment of methodological quality of studies and RCTs} The quality of the eligible RCTs was assessed systematically using the revised Cochrane risk-of-bias tool for randomized trials $2.0 .^{5}$ The 
present document reflects the consensus achieved during the group's deliberations. Agreement among the members of the group could be achieved for all sections, except for the randomization process (in particular concealment of allocation). Trials are listed by topic and in chronological order according to their respective recruitment period. Only published results were considered, and the longest follow-up was given priority. Published manuscripts, supplementary appendices, and protocols were reviewed for each trial.

The five following domains were assessed for their specific risk of bias: i) randomization process, ii) deviations from the intended

\section{Supplementary Table 6 Risk-of-bias judgments for all evaluated trials}

\begin{tabular}{|c|c|c|c|c|c|c|}
\hline & 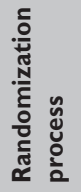 & 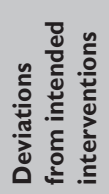 & 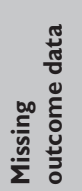 & 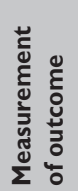 & 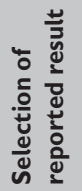 & $\begin{array}{l}\overline{\text { స్ }} \\
\text { ठ } \\
\text { ठ }\end{array}$ \\
\hline \multicolumn{7}{|l|}{ Treatment of aortic stenosis } \\
\hline \multicolumn{7}{|l|}{ PARTNER A - overall population* } \\
\hline Non-inferiority primary outcome at 5 years ${ }^{a}$ & $?$ & & & & + & \\
\hline \multicolumn{7}{|l|}{ CoreValve U.S. Pivotal High Risk } \\
\hline Non-inferiority primary outcome at 5 years (ESC) & + & & & & + & \\
\hline Non-inferiority primary outcome at 5 years (EACTS) & $?$ & & & & + & \\
\hline Superiority primary outcome at 1 year (ESC) & + & & & & + & \\
\hline Superiority primary outcome at 1 year (EACTS) & $?$ & & & & + & \\
\hline \multicolumn{7}{|l|}{ NOTION } \\
\hline Similarity primary outcome at 5 years (ESC) & + & + & & & + & + \\
\hline Similarity primary outcome at 5 years (EACTS) & $?$ & + & 1 & 4 & + & $?$ \\
\hline \multicolumn{7}{|l|}{ PARTNER 2} \\
\hline Non-inferiority primary outcome at 2 years (ESC) & + & $?$ & & & + & $?$ \\
\hline Non-inferiority primary outcome at 2 years (EACTS) & $?$ & $?$ & + & + & + & $?$ \\
\hline \multicolumn{7}{|l|}{ SURTAVI } \\
\hline Non-inferiority at 2 years (ESC) & + & $?$ & + & + & + & $?$ \\
\hline Non-inferiority at 2 years (EACTS) & $?$ & $?$ & + & + & + & $?$ \\
\hline \multicolumn{7}{|l|}{ PARTNER 3} \\
\hline Non-inferiority primary outcome at 2 years & $?$ & $?$ & + & + & + & $?$ \\
\hline Superiority primary outcome at 2 years ${ }^{\mathrm{a}}$ & $?$ & $?$ & & & + & \\
\hline \multicolumn{7}{|l|}{ Evolut Low Risk } \\
\hline Non-inferiority primary outcome at 2 years (ESC) & + & $?$ & + & + & + & $?$ \\
\hline Non-inferiority primary outcome at 2 years (EACTS) & $?$ & $?$ & + & + & + & $?$ \\
\hline \multicolumn{7}{|l|}{ Treatment of secondary mitral regurgitation } \\
\hline \multicolumn{7}{|l|}{ COAPT } \\
\hline Superiority primary outcome at 2 years (ESC) & + & $?$ & + & + & + & $?$ \\
\hline Superiority primary outcome at 2 years (EACTS) & $?$ & $?$ & + & + & + & $?$ \\
\hline Superiority secondary outcomes at 2 years (ESC) & + & $?$ & $?$ & $?$ & + & $?$ \\
\hline Superiority secondary outcomes at 2 years (EACTS) & $?$ & $?$ & $?$ & $?$ & + & $?$ \\
\hline \multicolumn{7}{|l|}{ MITRA-FR } \\
\hline Primary outcome at 2 years (ESC) & + & + & + & + & + & + \\
\hline Primary outcome at 2 years (EACTS) & $?$ & + & + & + & + & $?$ \\
\hline
\end{tabular}

ESC = delegates of the European Society of Cardiology; EACTS = delegates of the European Association for Cardio-Thoracic Surgery.

=low risk of bias; ? = some concerns; $=$ = high risk of bias

a Two separate statements are included where no consensus could be reached. Initials indicate the members of the group endorsing each risk-of-bias judgment. 
interventions, iii) missing outcome data, iv) measurement of outcome, and v) selection of reported results. For each domain, the overall risk of bias was derived according to pre-specified algorithms ${ }^{5}$ and quantified using three categories: low risk of bias, some concerns, and high risk of bias. Only domains with some concerns or high risk of bias will be discussed in the present document.

If appropriate, robustness to missing data was explored using a worst-case scenario that assumed no primary endpoint event in all missing patients of the control group [surgical aortic valve replacement (SAVR) or guideline-directed medical therapy (GDMT)] and the occurrence of an event in all patients of the experimental group [transcatheter aortic valve implantation (TAVI) or transcatheter mitral valve repair (TMVR)]. Supplementary Table 6 below summarizes the risk-of-bias judgments for all evaluated trials.

\section{Treatment of severe aortic stenosis}

\section{PARTNER A (5 years) ${ }^{6}$}

Domain 1: The group indicates that some concerns exist due to the lack of description of a central randomization process.

Domains 2, 3, and 4: A substantial proportion of the randomized patients did not undergo the intended procedure and were not followed up, particularly in the surgical group [38 patients (10.8\%) vs. $4(1.1 \%)$ in the transcatheter aortic valve replacement (TAVR) group]. The main reason for this was patient's decision not to undergo SAVR. In addition, there was an imbalance between the number of lost to follow-up (LTFU) at 5 years ( 9 patients in the TAVI group vs. 23 patients for SAVR). This translates into a high risk of bias for the overall population for non-inferiority at 5 years since the worst-case scenario suggests SAVR to be superior to TAVI. However, there is a clear access route by treatment interaction, with worse prognosis for transapical access to TAVI that needs to be considered when interpreting the trial results. The published hazard ratio for transapical access is 1.37 at 5 years [ $95 \%$ confidence interval $(\mathrm{Cl}) 0.98-1.92]$ whereas the published hazard ratio for transfemoral is 0.91 at 5 years $(95 \% \mathrm{Cl}, 0.72-1.14)$, $P$ for interaction $=0.05$, and the distinction is valid as randomization was stratified by intended access route.

\section{Overall risk of bias:}

High risk of bias regarding non-inferiority at 5 years for the overall population due to missing outcome data. Correct interpretation of the results requires distinction between transfemoral and transapical access; for transfemoral access TAVI and SAVR appeared similar at 5 years.

\section{CoreValve U.S. Pivotal High-Risk Trial (5 years) ${ }^{7}$}

Domain 1: The group was not able to reach an agreement concerning this domain. EACTS delegates indicate some concerns, as there is a theoretical risk of selection bias of unclear magnitude and direction because of stratification by site and blocking with randomly varied block sizes. In contrast, ESC delegates follow the published algorithm of the Cochrane risk-of-bias tool and classify this domain as low risk of bias, as they consider the potential for selection bias of a relevant magnitude through the combination of stratification by site and blocking to be small and of theoretical nature only.
Domains 2, 3, and 4: A substantial proportion of the randomized patients did not undergo the intended procedure and were not followed up, particularly in the surgical group [38 patients (9\%) vs. 4 (1\%) in the TAVI group]. The main reason for this was patient's consent withdrawal. In addition, there was an imbalance between the number of LTFU at 5 years ( 29 patients in the TAVI group vs. 48 patients for SAVR). This results in a high risk of bias for the overall population for both the non-inferiority and superiority at 5 years, since the worst-case scenario suggests SAVR to be superior to TAVI. In addition, there is a high risk of bias regarding previous claims of superiority at 1 year on the primary outcome.

\section{Overall risk of bias:}

High risk of bias regarding both non-inferiority at 1 and 5 years, as well as previous claims of superiority at 1 year due to missing outcome data.

\section{NOTION $^{8}$}

Domain 1: The group was not able to reach an agreement concerning this domain. EACTS delegates indicate some concerns, as there is a theoretical risk of selection bias of unclear magnitude and direction because of stratification by site and blocking with randomly varied block sizes. In contrast, ESC delegates follow the published algorithm of the Cochrane risk-of-bias tool and classify this domain as low risk of bias, as they consider the potential for selection bias of a relevant magnitude through the combination of stratification by site and blocking to be small and of theoretical nature only.

\section{Overall risk of bias:}

- The group was not able to reach an agreement.

- Low risk of bias regarding similarity of the primary endpoint according to ESC delegates; some concerns according to EACTS delegates.

\section{PARTNER $2^{9}$}

Domain 1: The group was not able to reach an agreement concerning this domain. EACTS delegates indicate some concerns, as there is a theoretical risk of selection bias of unclear magnitude and direction because of stratification by site and blocking with randomly varied block sizes. In contrast, ESC delegates follow the published algorithm of the Cochrane risk-of-bias tool and classify this domain as low risk of bias, as they consider the potential for selection bias of a relevant magnitude through the combination of stratification by site and blocking to be small and of theoretical nature only.

Domain 2: Some concerns exist because of imbalances in concomitant procedures [coronary artery bypass grafting (CABG) $14.5 \%$ vs. $3.9 \%$ percutaneous coronary intervention (PCl); $9.1 \%$ of patients had other surgical concomitant procedures]. This may have favoured the experimental group (TAVI) due to the incremental risk of stroke and death associated with concomitant surgical procedures, while differences in revascularization rates could also favour the control group (SAVR) due to the higher frequency of revascularization in this group. Importantly, this reflects clinical reality and may not explain the 
numerical difference of 18 neurological events in favour of TAVI observed at 2 years of follow-up.

Domains 3 and 4: Some of the randomized patients did not undergo the intended procedure and were not followed up, particularly in the surgical group [77 patients (7.5\%) vs. 17 (1.7\%) in the TAVI group]. The main reason for this was patient's decision not to undergo SAVR. In addition, there were an imbalance between the number of LTFU at 2 years ( 31 patients in the TAVI group vs. 45 patients for $\mathrm{SAVR}$ ). Despite these imbalances, there is a low risk of bias for the noninferiority analysis since even considering the worst-case scenario noninferiority was still demonstrated for the primary endpoint at 2 years.

\section{Overall risk of bias:}

Some concerns regarding non-inferiority of the primary outcome at 2 years due to imbalances in concomitant procedures.

\section{SURTAVI ${ }^{10}$}

Domain 1: The group was not able to reach an agreement concerning this domain. EACTS delegates indicate some concerns, as there is a theoretical risk of selection bias of unclear magnitude and direction because of stratification by site and blocking with randomly varied block sizes. In contrast, ESC delegates follow the published algorithm of the Cochrane risk-of-bias tool and classify this domain as low risk of bias, as they consider the potential for selection bias of a relevant magnitude through the combination of stratification by site and blocking to be small and of theoretical nature only.

Domain 2: Some concerns exist because of imbalances in concomitant procedures [CABG (22.1\%) vs. PCl (14.5\%); cardiac ablation (8\%); and root enlargement (1.6\%)]. This may have favoured the experimental group (TAVI) due to the incremental risk of stroke and death associated with concomitant surgical procedures, while differences in revascularization rates could also favour the control group (SAVR) due to the higher frequency of revascularization in this group. Importantly, this reflects clinical reality and is unlikely to explain differences observed at 2 years of follow-up.

Domains 3 and 4: A substantial proportion of the randomized patients did not undergo the intended procedure and were not followed up, particularly in the surgical group [71 patients (8.1\%) vs. 15 (1.7\%) in the TAVI group]. Despite these imbalances, there is a low risk of bias for the non-inferiority analysis since even considering the worst-case scenario, non-inferiority was still demonstrated for the primary endpoint at 2 years.

\section{Overall risk of bias:}

Some concerns regarding non-inferiority of the primary outcome at 2 years due to imbalances in concomitant procedures.

\section{PARTNER $3^{11}$}

Domain 1: The group agreed that there are some concerns regarding undermining of concealment due to stratification by site and block randomization with a fixed block size of four. If this had been known to sites, allocation would have been fully predictable for sites in case of a difference of 2 participants between groups. Therefore, there is a theoretical possibility of selection bias of unclear magnitude and direction.

Domain 2: Some concerns exist because of imbalances in left atrial appendage (LAA) ligation [43 patients (9.5\%)] as concomitant procedure (which was allowed according to the protocol) and Maze [22 patients (4.8\%)] as non-protocol co-intervention (which was a protocol deviation). This may have favoured the experimental group (TAVI) due to the incremental risk of stroke and death associated with concomitant surgical procedures, while differences in revascularization rates could also favour the control group (SAVR) due to the higher frequency of revascularization in this group. No appropriate statistical method was used to account for these discrepancies. However, both procedures seem unlikely to be associated with a greater than $5 \%$ risk of peri-procedural stroke ${ }^{12,13}$ and therefore may not explain the difference of 8 strokes in favour of TAVI observed at 1 year.

Domains 3 and 4: A substantial proportion of the randomized patients did not undergo the intended procedure and were not followed up, particularly in the surgical group [43 patients (8.6\%) vs. 7 (1.4\%) in the TAVI group]. The main reason for this was patients' decisions not to undergo SAVR. The number of LTFU at 1 year was low for both groups ( 3 patients in the TAVI group vs. 12 patients for SAVR). Despite imbalances in the number of patients who did not receive the intended treatment, there is a low risk of bias for the noninferiority analysis since even considering the worst-case scenario noninferiority was still demonstrated for the primary endpoint at 1 year. In contrast, there is a high risk of bias for the superiority analysis since the $95 \% \mathrm{Cl}$ of the primary outcome crosses the line of no difference in the worst-case scenario.

\section{Overall risk of bias:}

Some concerns regarding non-inferiority on the primary outcome at 2 years due to the use of a fixed block size of 4 for the randomization process and imbalances in concomitant procedures; high risk of bias regarding superiority on the primary outcome at 2 years due to missing data in the patients who did not receive the intended intervention.

\section{Evolut low risk ${ }^{14}$}

Domain 1: The group was not able to reach an agreement concerning this domain. EACTS delegates indicate some concerns, as there is a theoretical risk of selection bias of unclear magnitude and direction because of stratification by site and blocking with randomly varied block sizes. In contrast, ESC delegates follow the published algorithm of the Cochrane risk-of-bias tool and classify this domain as low risk of bias, as they consider the potential for selection bias of a relevant magnitude through the combination of stratification by site and blocking to be small and of theoretical nature only.

Domain 2: Some concerns exist because of imbalances in LAA ligation [42 patients (6.2\%)] and Maze [24 patients (3.5\%)] as concomitant procedures (which were both allowed according to the protocol). This may have favoured the experimental group (TAVI) due to the incremental risk of stroke and death associated with concomitant surgical procedures, while differences in revascularization rates could 
also favour the control group (SAVR) due to the higher frequency of revascularization in this group. No appropriate statistical method was used to account for these discrepancies. However, both procedures are unlikely to be associated with a greater than $5 \%$ risk of peri-procedural stroke $\mathrm{e}^{12,13}$ and do not therefore explain differences between groups.

Domains 3 and 4: A substantial proportion of the randomized patients did not undergo the intended procedure and were not followed up, particularly in the surgical group [53 patients (7.2\%) vs. 12 (1.6\%) in the TAVI group]. In addition, there was an imbalance between the number of LTFU at 2 years ( 9 patients in the TAVI group vs. 27 patients for SAVR). Despite imbalances in the number of patients who did not receive the intended treatment, there is a low risk of bias for the non-inferiority analysis since even considering the worst-case scenario non-inferiority was still demonstrated for the primary endpoint at 2 years.

\section{Overall risk of bias:}

Some concerns regarding non-inferiority on the primary outcome due to imbalances in concomitant procedures.

\section{Treatment of secondary mitral regurgitation COAPT $^{15}$}

Domain 1: The group was not able to reach an agreement concerning this domain. EACTS delegates indicate some concerns, as there is a theoretical risk of selection bias of unclear magnitude and direction because of stratification by site and blocking with randomly varied block sizes. In contrast, ESC delegates follow the published algorithm of the Cochrane risk-of-bias tool and classify this domain as low risk of bias, as they consider the potential for selection bias of a relevant magnitude through the combination of stratification by site and blocking to be small and of theoretical nature only.

Domain 2: The group acknowledges that some concerns exist due to the significantly lower use of angiotensin-converting enzyme inhibitors (ACEI), angiotensin receptor blockers (ARB), or angiotensin receptor-neprilysin inhibitors (ARNI) at baseline in the patients of the GDMT group ( $62.8 \%$ vs. $71.5 \%$ in the TMVR group). This could indicate failure to implement medical treatment in the control group and may have favoured the TMVR group. However, considerable efforts were made to optimize GDMT at study entry through control by a clinical eligibility committee, the evolution of the medical therapy was strictly monitored for both groups, and the percentage of patients with major changes was low in both groups. In addition, patients in the control group had worse heart failure symptoms as reflected by New York Heart Association (NYHA) classes III and IV.

Domains 3 and 4: There was an imbalance between the number of LTFU at 2 years ( 25 patients in the TMVR group vs. 47 patients in the GDMT group). An attempt to account for missing data was made using multiple imputation, but resulting estimates may not be robust. There was a low risk of bias regarding superiority on the primary outcome as findings were robust to missing data considering the worst-case scenario. In contrast, some concerns exist for superiority on secondary outcomes due to missing data and lower use of ACEI, $\mathrm{ARB}$, or $\mathrm{ARNI}$ at baseline.

\section{Overall risk of bias:}

Some concerns regarding superiority on the primary outcome due to lower use of ACEI, ARB, or ARNI at baseline; some concerns for superiority on secondary outcomes due to missing data and lower use of $\mathrm{ACEI}, \mathrm{ARB}$, or ARNI at baseline.

\section{MITRA-FR ${ }^{16}$}

Domain 1: The group was not able to reach an agreement concerning this domain. EACTS delegates indicate some concerns, as there is a theoretical risk of selection bias of unclear magnitude and direction because of stratification by site and blocking with randomly varied block sizes. In contrast, ESC delegates follow the published algorithm of the Cochrane risk-of-bias tool and classify this domain as low risk of bias, as they consider the potential for selection bias of a relevant magnitude through the combination of stratification by site and blocking to be small and of theoretical nature only.

Domains 2, 3, and 4: Low concerns for the primary endpoint $(99 \%$ follow-up). Analyses of secondary endpoints were deemed inconclusive due to the high level of missing data.

\section{Overall risk of bias:}

- The group was not able to reach an agreement.

- Low risk of bias according to the ESC delegates regarding the superiority analysis of the primary outcome; some concerns according to the EACTS delegates regarding the superiority analysis of the primary outcome due to the randomization process. Consensus that analyses of secondary endpoints were inconclusive due to the high level of missing data.

\section{Mitral regurgitation}

\section{Supplementary Table 7 Main inclusion/exclusion crite- ria suggesting an increased chance of responding to TEER in patients with SMR}

\section{Inclusion criteria:}

- Severe SMR

- Symptomatic heart failure (NYHA class II, III or ambulatory IV) despite optimized GDMT

- LVEF 20-50\%

- LV end-systolic diameter $\leq 70 \mathrm{~mm}$

- At least one heart failure hospitalization within the previous year or increased natriuretic peptide levels

- Anatomy judged suitable for TEER

Exclusion criteria:

- Severe disability/frailty

- Hypertrophic cardiomyopathy, restrictive cardiomyopathy, constrictive pericarditis, or any other structural heart disease causing heart failure other than dilated cardiomyopathy of either ischemic or nonischaemic etiology

- Infiltrative cardiomyopathies (e.g. amyloidosis, haemochromatosis, sarcoidosis) 
- Estimated SPAP $>70 \mathrm{mmHg}$ assessed by echocardiography or right heart catheterization

- Haemodynamic instability defined as systolic pressure $<90 \mathrm{mmHg}$ with or without afterload reduction, cardiogenic shock or the need for inotropic support or intra-aortic balloon pump or other haemodynamic support device

- Physical evidence of right-sided congestive heart failure with echocardiographic evidence of moderate or severe RV dysfunction

- Mitral valve orifice area $<4.0 \mathrm{~cm}^{2}$ by site-assessed TTE

- Coronary, aortic or tricuspid valve disease requiring surgery

GDMT = guideline-directed medical therapy; $L V=$ left ventricle/left ventricular; $L V E F=$ left ventricular ejection fraction; NYHA $=$ New York Heart Association; $\mathrm{RV}=$ right ventricle/right ventricular; $\mathrm{SMR}=$ secondary; $\mathrm{SPAP}=$ systolic pulmonary arterial pressure; TTE $=$ transthoracic echocardiography; $T E E R=$ transcatheter edge-to-edge repair.

Adapted from Mack MJ et al., Cardiovascular outcomes assessment of the MitraClip in patients with heart failure and secondary mitral regurgitation: design and rationale of the COAPT trial. Am Heart J. 2018;205:1 -11, Copyright (2018), with permission from Elsevier. ${ }^{17}$

\section{Tricuspid regurgitation}

There is no supplementary material for this section.

\section{Tricuspid stenosis}

There is no supplementary material for this section.

\section{Combined and multiple-valve diseases}

There is no supplementary material for this section.

\section{Mitral stenosis}

Supplementary Table 8 Echocardiographic scores used for assessing the feasibility of percutaneous mitral commissurotomy: Wilkins score, Cormier score, and Echo score 'Revisited'

\begin{tabular}{|c|c|c|c|c|}
\hline \multicolumn{5}{|c|}{ Assessment of mitral valve anatomy according to the Wilkins score ${ }^{18}$} \\
\hline Grade & Mobility & Thickening & Calcification & Subvalvular thickening \\
\hline 1 & $\begin{array}{l}\text { Highly mobile valve with only leaflet } \\
\text { tips restricted }\end{array}$ & $\begin{array}{l}\text { Leaflets near normal in } \\
\text { thickness }(4-5 \mathrm{~mm})\end{array}$ & $\begin{array}{l}\text { A single area of increased echo } \\
\text { brightness }\end{array}$ & $\begin{array}{l}\text { Minimal thickening just below } \\
\text { the mitral leaflets }\end{array}$ \\
\hline 2 & $\begin{array}{l}\text { Leaflet mid and base portions have } \\
\text { normal mobility }\end{array}$ & $\begin{array}{l}\text { Mid leaflets normal, considerable } \\
\text { thickening of margins }(5-8 \mathrm{~mm})\end{array}$ & $\begin{array}{l}\text { Scattered areas of brightness } \\
\text { confined to leaflet margins }\end{array}$ & $\begin{array}{l}\text { Thickening of chordal struc- } \\
\text { tures extending to one third of } \\
\text { the chordal length }\end{array}$ \\
\hline 3 & $\begin{array}{l}\text { Valve continues to move forward in } \\
\text { diastole, mainly from the base }\end{array}$ & $\begin{array}{l}\text { Thickening extending through } \\
\text { the entire leaflet }(5-8 \mathrm{~mm})\end{array}$ & $\begin{array}{l}\text { Brightness extending into the } \\
\text { mid portions of the leaflets }\end{array}$ & $\begin{array}{l}\text { Thickening extended to distal } \\
\text { third of the chords }\end{array}$ \\
\hline 4 & $\begin{array}{l}\text { No or minimal forward movement } \\
\text { of the leaflets in diastole }\end{array}$ & $\begin{array}{l}\text { Considerable thickening of } \\
\text { all leaflet tissue }(>8-10 \mathrm{~mm})\end{array}$ & $\begin{array}{l}\text { Extensive brightness through- } \\
\text { out much of the leaflet tissue }\end{array}$ & $\begin{array}{l}\text { Extensive thickening and short- } \\
\text { ening of all chordal structures } \\
\text { extending down to the papillary } \\
\text { muscles }\end{array}$ \\
\hline
\end{tabular}

The total score is the sum of the four items and ranges between 4 and 16.

\begin{tabular}{|c|c|c|}
\hline \multicolumn{3}{|c|}{ Assessment of mitral valve anatomy according to the Cormier score ${ }^{19}$} \\
\hline Echocardiographic group & Mitral valve anatomy & \\
\hline Group 1 & $\begin{array}{l}\text { Pliable non-calcified anterior mitral leaflet and mild subvalvular disease } \\
\text { (i.e. thin chordae } \geq 10 \mathrm{~mm} \text { long) }\end{array}$ & \\
\hline Group 2 & $\begin{array}{l}\text { Pliable non-calcified anterior mitral leaflet and severe subvalvular disease } \\
\text { (i.e. thickened chordae }<10 \mathrm{~mm} \text { long) }\end{array}$ & \\
\hline Group 3 & $\begin{array}{l}\text { Calcification of mitral valve of any extent, as assessed by fluoroscopy, } \\
\text { whatever the state of subvalvular apparatus }\end{array}$ & \\
\hline \multicolumn{3}{|c|}{ Echo score 'Revisited' for immediate outcome prediction ${ }^{20}$} \\
\hline Echocardiographic variables & Points for score (0 to 11) & \\
\hline Mitral valve area $\leq 1 \mathrm{~cm}^{2}$ & 2 & 5 \\
\hline Maximum leaflet displacement $\leq 12 \mathrm{~mm}$ & 3 & \\
\hline Commissural area ratio $\geq 1.25$ & 3 & \\
\hline Subvalvular involvement & 3 & 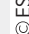 \\
\hline
\end{tabular}

Risk groups for Echo score 'Revisited': low (score 0-3); intermediate (score 4-5); high (score 6-11).

Reproduced from Baumgartner H et al. 2017 ESC/EACTS Guidelines for the management of valvular heart disease. Eur Heart J 2017;38:2739-2791, by permission of Oxford University Press on behalf of the European Society of Cardiology. ${ }^{21}$ 


\section{Prosthetic valves}

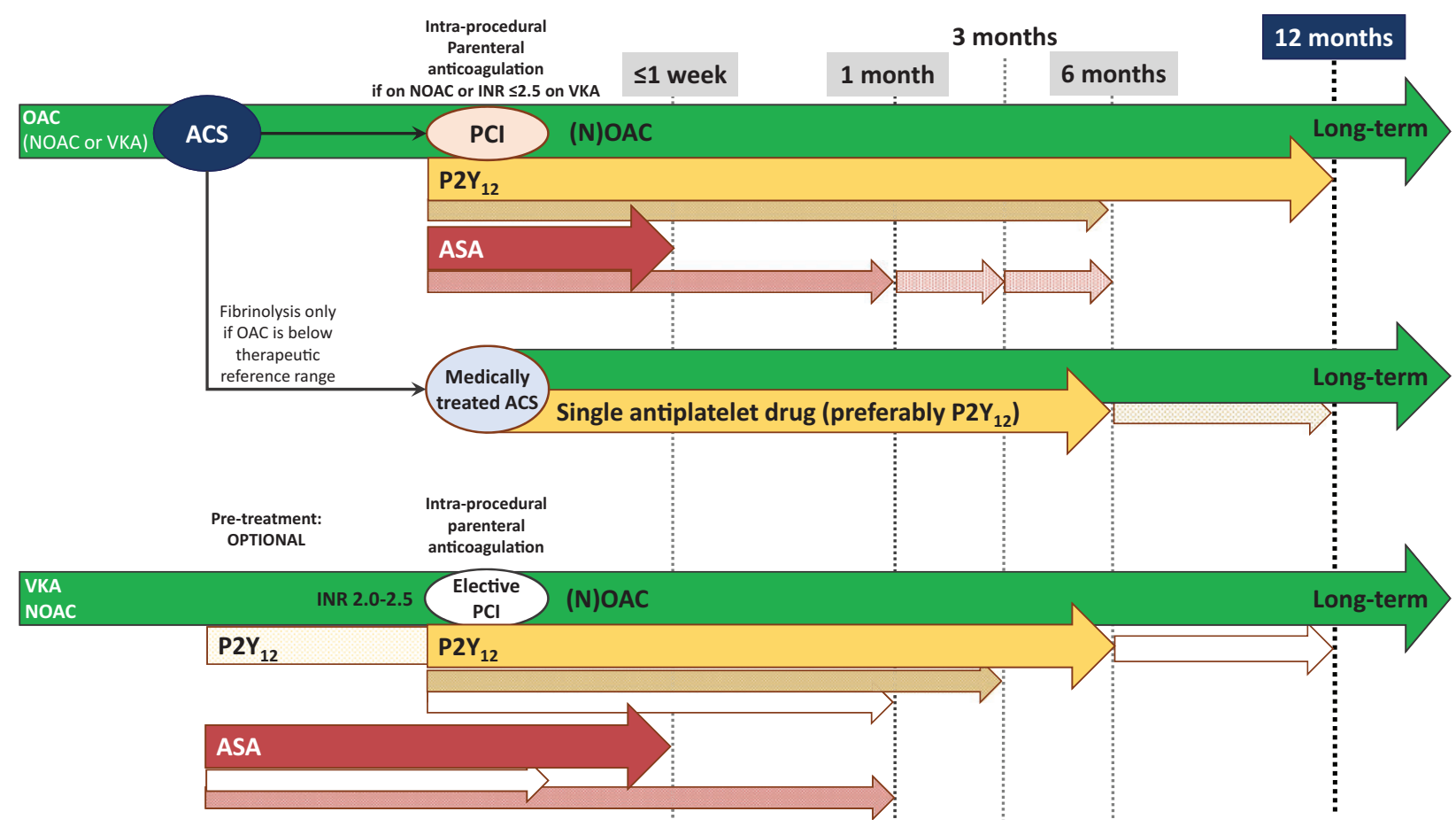

Supplementary Figure 2 Peri- and post-procedural management of antithrombotic therapy in patients with indication to OAC and ACS/PCI. $\mathrm{ACS}=$ acute coronary syndrome; $\mathrm{ASA}=$ acetylsalicylic acid; INR = international normalized ratio; NOAC = non-vitamin $\mathrm{K}$ antagonist oral anticoagulant; $\mathrm{OAC}=$ oral anticoagulation; $\mathrm{PCl}=$ percutaneous coronary intervention; VKA= vitamin $\mathrm{K}$ antagonist. Adapted from Hindricks $\mathrm{G}$ et al., 2020 ESC Guidelines for the diagnosis and management of atrial fibrillation developed in collaboration with the European Association for Cardio-Thoracic Surgery (EACTS). Eur Heart J 2021;42:373-498. Copyright (2021) by permission of Oxford University Press on behalf of the European Society of Cardiology. ${ }^{22}$

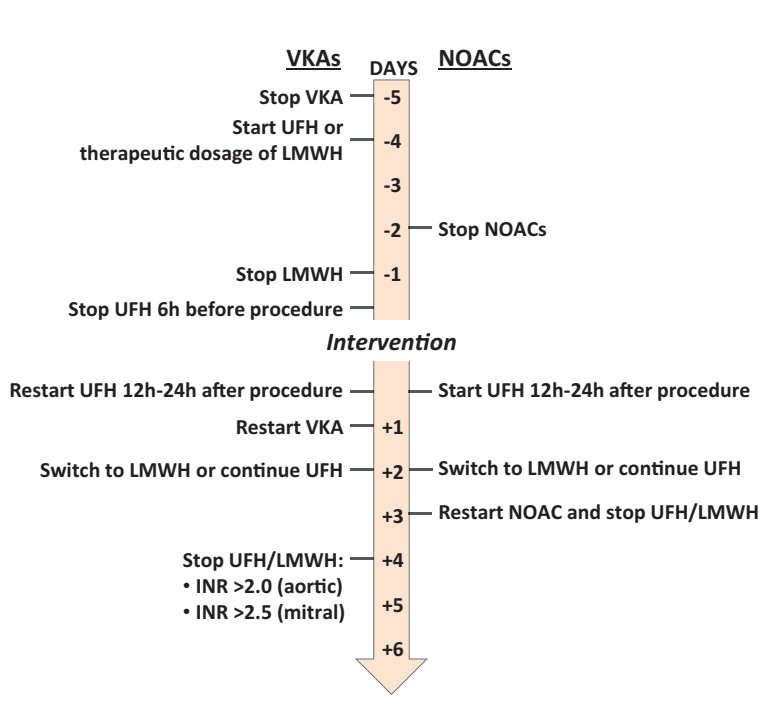

Supplementary Figure 3 Management of OAC in patients with an indication for preoperative bridging. INR= international normalized ratio; LMWH = low-molecular-weight heparin; NOAC = non-vitamin $\mathrm{K}$ antagonist oral anticoagulant; $\mathrm{OAC}=$ oral anticoagulation; UFH = unfractionated heparin; $\mathrm{VKA}=$ vitamin $\mathrm{K}$ antagonist. ${ }^{\mathrm{a} B}$ Bidging with UFH/LMWH should start when INR values are below specific therapeutic ranges. ${ }^{\mathrm{b}} \mathrm{Discontinuation}$ should be prolonged to $>72 \mathrm{~h}$ if creatinine clearance is $50-79 \mathrm{~mL} / \mathrm{min} / 1.73 \mathrm{~m}^{2}$ or $>96 \mathrm{~h}$ if creatinine clearance is $<50 \mathrm{~mL} / \mathrm{min} / 1.73 \mathrm{~m}$. Of note, VKA should be stopped 5 days before intervention if warfarin is used but only 3 days in the case of acenocumarol. Reproduced from Sousa-Uva M et al., 2017 EACTS Guidelines on perioperative medication in adult cardiac surgery. Eur J Cardiothorac Surg 2018;53:5-33, by permission of Oxford University Press on behalf of the European Association for Cardio-Thoracic Surgery. ${ }^{23}$ 


\section{Management during non-cardiac surgery}

There is no supplementary material for this section.

\section{Management during pregnancy}

There is no supplementary material for this section.

\section{Key messages}

There is no supplementary material for this section.

\section{Gaps in evidence}

There is no supplementary material for this section.

\section{To Do and Not to Do}

There is no supplementary material for this section.

\section{References}

1. Puri R, lung B, Cohen DJ, Rodes-Cabau J. TAVI or no TAVI: identifying patients unlikely to benefit from transcatheter aortic valve implantation. Eur Heart J 2016;37:2217-2225.

2. Afilalo J. The Clinical Frailty Scale: Upgrade Your Eyeball Test. Circulation 2017; 135:2025-2027.

3. Afilalo J, Lauck S, Kim DH, Lefevre T, Piazza N, Lachapelle K, Martucci G, Lamy A, Labinaz M, Peterson MD, Arora RC, Noiseux N, Rassi A, Palacios IF, Genereux P, Lindman BR, Asgar AW, Kim CA, Trnkus A, Morais JA, Langlois Y, Rudski LG, Morin JF, Popma JJ, Webb JG, Perrault LP. Frailty in older adults undergoing aortic valve replacement: the FRAILTY-AVR Study. J Am Coll Cardiol 2017;70:689-700.

4. Gavazzoni M, Taramasso M, Zuber M, Russo G, Pozzoli A, Miura M, Maisano F. Conceiving MitraClip as a tool: percutaneous edge-to-edge repair in complex mitral valve anatomies. Eur Heart J Cardiovasc Imaging 2020;21:1059-1067.

5. Sterne JAC, Savovic J, Page MJ, Elbers RG, Blencowe NS, Boutron I, Cates CJ, Cheng HY, Corbett MS, Eldridge SM, Emberson JR, Hernan MA, Hopewell S, Hrobjartsson A, Junqueira DR, Juni P, Kirkham Jj, Lasserson T, Li T, McAleenan A, Reeves BC, Shepperd S, Shrier I, Stewart LA, Tilling K, White IR, Whiting PF, Higgins JPT. RoB 2: a revised tool for assessing risk of bias in randomised trials. BMJ 2019;366:14898.

6. Mack MJ, Leon MB, Smith CR, Miller DC, Moses JW, Tuzcu EM, Webb JG, Douglas PS, Anderson WN, Blackstone EH, Kodali SK, Makkar RR, Fontana GP, Kapadia S, Bavaria J, Hahn RT, Thourani VH, Babaliaros V, Pichard A, Herrmann HC, Brown DL, Williams M, Akin J, Davidson MJ, Svensson LG, PARTNER 1 Trial Investigators. 5-Year outcomes of transcatheter aortic valve replacement or surgical aortic valve replacement for high surgical risk patients with aortic stenosis (PARTNER 1): a randomised controlled trial. Lancet 2015;385:2477-2484.

7. Gleason TG, Reardon MJ, Popma JJ, Deeb GM, Yakubov SJ, Lee JS, Kleiman NS, Chetcuti S, Hermiller JB, Jr., Heiser J, Merhi W, Zorn GL, 3rd, Tadros P, Robinson N, Petrossian G, Hughes GC, Harrison JK, Conte JV, Mumtaz M, Oh JK, Huang J, Adams DH, CoreValve US Pivotal High Risk Trial Clinical Investigators. 5-Year outcomes of self-expanding transcatheter versus surgical aortic valve replacement in high-risk patients. J Am Coll Cardiol 2018;72:2687-2696.

8. Thyregod HGH, Ihlemann N, Jorgensen TH, Nissen H, Kjeldsen BJ, Petursson P, Chang Y, Franzen OW, Engstrom T, Clemmensen P, Hansen PB, Andersen LW, Steinbruchel DA, Olsen PS, Sondergaard L. Five-Year clinical and echocardiographic outcomes from the Nordic Aortic Valve Intervention (NOTION) randomized clinical trial in lower surgical risk patients. Circulation 2019;139:2714-2723.

9. Leon MB, Smith CR, Mack MJ, Makkar RR, Svensson LG, Kodali SK, Thourani VH, Tuzcu EM, Miller DC, Herrmann HC, Doshi D, Cohen DJ, Pichard AD, Kapadia S, Dewey T, Babaliaros V, Szeto WY, Williams MR, Kereiakes D, Zajarias A, Greason KL, Whisenant BK, Hodson RW, Moses JW, Trento A, Brown DL, Fearon WF, Pibarot P, Hahn RT, Jaber WA, Anderson WN, Alu MC,
Webb JG, PARTNER 2 Investigators. Transcatheter or surgical aortic-valve replacement in intermediate-risk patients. N Engl J Med 2016;374:1609-1620.

10. Reardon MJ, Van Mieghem NM, Popma JJ, Kleiman NS, Sondergaard L, Mumtaz M, Adams DH, Deeb GM, Maini B, Gada H, Chetcuti S, Gleason T, Heiser J, Lange R, Merhi W, Oh JK, Olsen PS, Piazza N, Williams M, Windecker S, Yakubov SJ, Grube E, Makkar R, Lee JS, Conte J, Vang E, Nguyen H, Chang Y, Mugglin AS, Serruys PW, Kappetein AP, SURTAVI Investigators. Surgical or transcatheter aortic-valve replacement in intermediate-risk patients. N Engl J Med 2017;376:1321-1331.

11. Mack MJ, Leon MB, Thourani VH, Makkar R, Kodali SK, Russo M, Kapadia SR, Malaisrie SC, Cohen DJ, Pibarot P, Leipsic J, Hahn RT, Blanke P, Williams MR, McCabe JM, Brown DL, Babaliaros V, Goldman S, Szeto WY, Genereux P, Pershad A, Pocock SJ, Alu MC, Webb JG, Smith CR, PARTNER 3 Investigators. Transcatheter aortic-valve replacement with a balloon-expandable valve in lowrisk patients. N Engl J Med 2019;380:1695-1705.

12. Healey JS, Crystal E, Lamy A, Teoh K, Semelhago L, Hohnloser SH, Cybulsky I, Abouzahr L, Sawchuck C, Carroll S, Morillo C, Kleine P, Chu V, Lonn E, Connolly SJ. Left Atrial Appendage Occlusion Study (LAAOS): results of a randomized controlled pilot study of left atrial appendage occlusion during coronary bypass surgery in patients at risk for stroke. Am Heart J 2005;150:288-293.

13. Ad N, Holmes SD, Roberts HG, Jr., Rankin JS, Badhwar V. Surgical treatment for stand-alone atrial fibrillation in North America. Ann Thorac Surg 2020;109:745-752.

14. Popma J], Deeb GM, Yakubov SJ, Mumtaz M, Gada H, O'Hair D, Bajwa T, Heiser JC, Merhi W, Kleiman NS, Askew J, Sorajja P, Rovin J, Chetcuti SJ, Adams DH, Teirstein PS, Zorn GL, 3rd, Forrest JK, Tchetche D, Resar J, Walton A, Piazza N, Ramlawi B, Robinson N, Petrossian G, Gleason TG, Oh JK, Boulware MJ, Qiao $\mathrm{H}$, Mugglin AS, Reardon MJ, Evolut Low Risk Trial Investigators. Transcatheter aortic-valve replacement with a self-expanding valve in low-risk patients. N Engl J Med 2019;380:1706-1715.

15. Stone GW, Lindenfeld J, Abraham WT, Kar S, Lim DS, Mishell JM, Whisenant B, Grayburn PA, Rinaldi M, Kapadia SR, Rajagopal V, Sarembock IJ, Brieke A, Marx SO, Cohen DJ, Weissman NJ, Mack MJ, COAPT Investigators. Transcatheter mitralvalve repair in patients with heart failure. N Engl J Med 2018;379:2307-2318.

16. Obadia JF, Messika-Zeitoun D, Leurent G, lung B, Bonnet G, Piriou N, Lefevre T, Piot C, Rouleau F, Carrie D, Nejjari M, Ohlmann P, Leclercq F, Saint Etienne C, Teiger E, Leroux L, Karam N, Michel N, Gilard M, Donal E, Trochu JN, Cormier B, Armoiry X, Boutitie F, Maucort-Boulch D, Barnel C, Samson G, Guerin P, Vahanian A, Mewton N, MITRA-FR Investigators. Percutaneous repair or medical treatment for secondary. N Engl J Med 2018;379:2297-2306.

17. Mack MJ, Abraham WT, Lindenfeld J, Bolling SF, Feldman TE, Grayburn PA, Kapadia SR, McCarthy PM, Lim DS, Udelson JE, Zile MR, Gammie JS, Gillinov AM, Glower DD, Heimansohn DA, Suri RM, Ellis JT, Shu Y, Kar S, Weissman NJ, Stone GW. Cardiovascular outcomes assessment of the MitraClip in patients with heart failure and secondary : design and rationale of the COAPT trial. Am Heart J 2018;205:1-11.

18. Bonow RO, O'Gara PT, Adams DH, Badhwar V, Bavaria JE, Elmariah S, Hung JW, Lindenfeld J, Morris A, Satpathy R, Whisenant B, Woo YJ. 2019 AATS/ACC/SCAI/ STS Expert Consensus Systems of Care Document: operator and institutional recommendations and requirements for transcatheter mitral valve intervention: a joint report of the American Association for Thoracic Surgery, the American College of Cardiology, the Society for Cardiovascular Angiography and Interventions, and the Society of Thoracic Surgeons. J Am Coll Cardiol 2020;76:96-117.

19. Bouleti C, lung B, Himbert D, Brochet E, Messika-Zeitoun D, Detaint D, Garbarz E, Cormier B, Vahanian A. Reinterventions after percutaneous mitral commissurotomy during long-term follow-up, up to 20 years: the role of repeat percutaneous mitral commissurotomy. Eur Heart J 2013;34:1923-1930.

20. Nunes MC, Tan TC, Elmariah S, do Lago R, Margey R, Cruz-Gonzalez I, Zheng $\mathrm{H}$, Handschumacher MD, Inglessis I, Palacios IF, Weyman AE, Hung J. The Echo score revisited: impact of incorporating commissural morphology and leaflet displacement to the prediction of outcome for patients undergoing percutaneous mitral valvuloplasty. Circulation 2014;129:886-895.

21. Baumgartner H, Falk V, Bax J, De Bonis M, Hamm C, Holm PJ, lung B, Lancellotti $P$, Lansac E, Rodriguez Munoz D, Rosenhek R, Sjogren J, Tornos Mas P, Vahanian A, Walther T, Wendler O, Windecker S, Zamorano JL, ESC Scientific Document Group. 2017 ESC/EACTS Guidelines for the management of valvular heart disease. Eur Heart J 2017;38:2739-2791.

22. Hindricks G, Potpara T, Dagres N, Arbelo E, Bax JJ, Blomstrom-Lundqvist C, Boriani G, Castella M, Dan GA, Dilaveris PE, Fauchier L, Filippatos G, Kalman JM, La Meir M, Lane DA, Lebeau JP, Lettino M, Lip GYH, Pinto FJ, Thomas GN, Valgimigli M, Van Gelder IC, Van Putte BP, Watkins CL, ESC Scientific Document Group. 2020 ESC Guidelines for the diagnosis and management of atrial fibrillation developed in collaboration with the European Association for Cardio-Thoracic Surgery (EACTS). Eur Heart J 2021;42:373-498.

23. Sousa-Uva M, Head SJ, Milojevic M, Collet JP, Landoni G, Castella M, Dunning J, Gudbjartsson T, Linker NJ, Sandoval E, Thielmann M, Jeppsson A, Landmesser U. 2017 EACTS Guidelines on perioperative medication in adult cardiac surgery. Eur J Cardiothorac Surg 2018;53:5-33. 